\title{
Energy End-Use Intensities in Commercial Buildings
}

September 1994

\author{
Energy Information Administration \\ Office of Energy Markets and End Use \\ U.S. Department of Energy \\ Washington, DC 20585
}

This report was prepared by the Energy Information Administration, the independent statistical and analytical agency within the Department of Energy. The information contained herein should not be construed as advocating or reflecting any policy position of the Department of Energy or any other organization. 


\section{Contacts}

This publication was prepared under the general direction of W. Calvin Kilgore, Director of the Office of Energy Markets and End Use (202/586-1617), and Lynda T. Carlson, Director of the Energy End Use and Integrated Statistics Division (EEUISD) (202/586-1112). Specific information regarding the contents or preparation of this publication can be obtained from Dwight K. French, Chief of the Transportation and Industrial Branch (202/5861126). Questions regarding the Commercial Buildings Energy Consumption Survey (CBECS) may be referred to Martha M. Johnson, Survey Manager (202/586-1135). The FAX number for all EEUISD personnel is 202/586-0018.

Detailed technical questions on the topics in this publication may be referred to the following members of EEUISD:

$\begin{array}{lll}\text { Eugene M. Burns } & \text { Principal Author, End-Use Estimates } & \text { (202) 586-1125 } \\ \text { Jay K. Olsen } & \text { Data Imputation (other than end-uses) } & \text { (202) } 586-1137\end{array}$

The following persons also made important contributions to this publication:
Vicki Moorhead
Table Preparation
(202) $586-1133$
Hattie Ramseur
Report Production
(202) $586-1124$

This report was undertaken in response to CBECS customer requests for more information on how energy is used in buildings, which was an overall theme of the 1992 User Needs Study. (See UserNeeds Study for the 1992 Commercial Buildings Energy Consumption Survey, DOE/ELA-0555(92)/4 (Washington, DC, September 1992).) This is EIA's first report to present data on how much energy is used for heating, cooling, lighting, and other end uses in commercial buildings. We encourage comments from our readers. Feedback from the readers of this report will allow us to enhance our methodology and presentation as we undertake the end-use estimates based on the 1992 CBECS. Comments and suggestions regarding end-use estimates should be addressed to Eugene M. Burns by phone at (202) 5861125, by FAX at (202) 586-0018, or by e-mail at eburns@eia.doe.gov. The mailing address is:

EI-632, Mail Stop 2G-090

1000 Independence Avenue, SW

Washington, DC 20585. 


\section{DISCLAIMER}

This report was prepared as an account of work sponsored by an agency of the United States Government. Neither the United States Government nor any agency thereof, nor any of their employees, make any warranty, express or implied, or assumes any legal liability or responsibility for the accuracy, completeness, or usefulness of any information, apparatus, product, or process disclosed, or represents that its use would not infringe privately owned rights. Reference herein to any specific commercial product, process, or service by trade name, trademark, manufacturer, or otherwise does not necessarily constitute or imply its endorsement, recommendation, or favoring by the United States Government or any agency thereof. The views and opinions of authors expressed herein do not necessarily state or reflect those of the United States Government or any agency thereof. 


\section{DISCLAIMER}

\section{Portions of this document may be illegible in electronic image products. Images are produced from the best available original document.}




\section{Contents}

Executive Summary $\ldots \ldots \ldots \ldots \ldots \ldots \ldots \ldots \ldots \ldots \ldots \ldots \ldots \ldots \ldots \ldots \ldots \ldots \ldots$

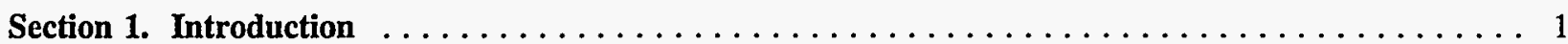

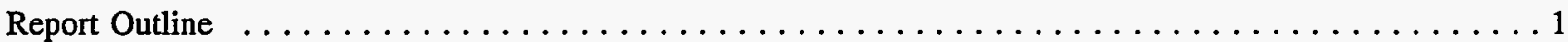

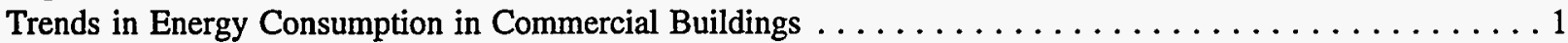

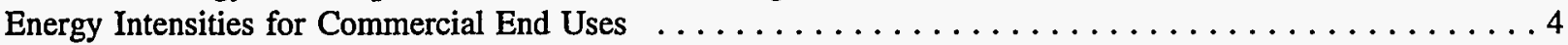

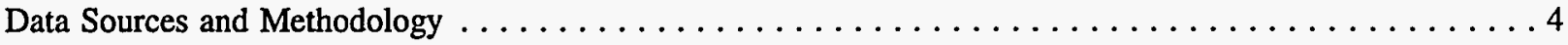

Section 2. Energy Use in Commercial Buildings $\ldots \ldots \ldots \ldots \ldots \ldots \ldots \ldots \ldots \ldots \ldots \ldots \ldots \ldots$

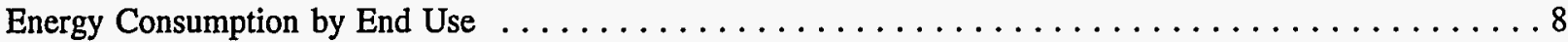

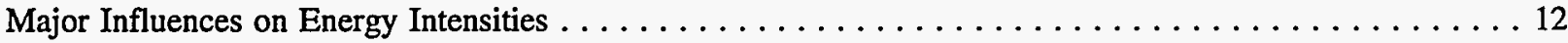

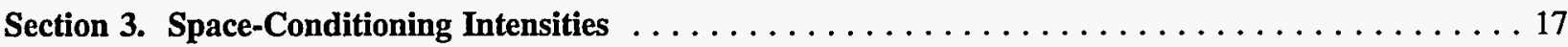

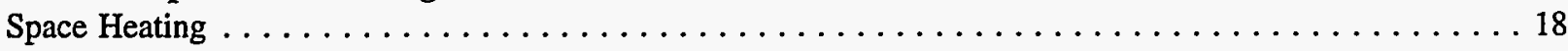

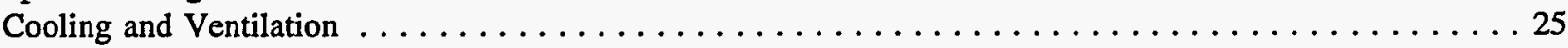

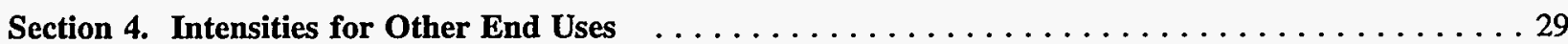

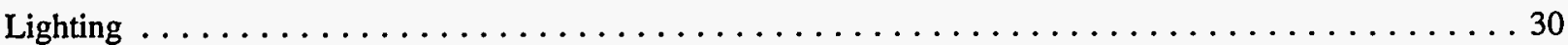

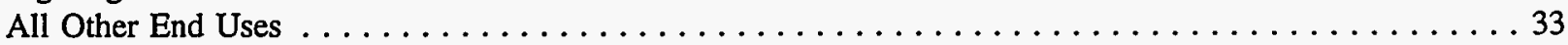

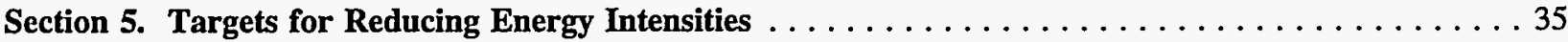

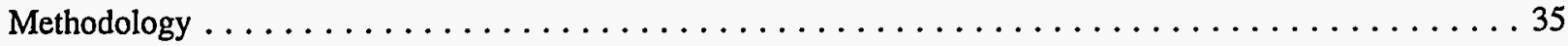

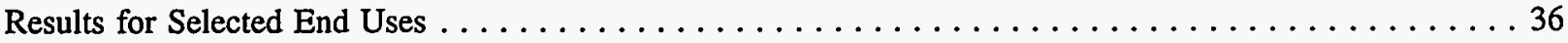

Appendices

A. Development of End-Use Intensity Estimates $\ldots \ldots \ldots \ldots \ldots \ldots \ldots \ldots \ldots \ldots \ldots \ldots \ldots \ldots \ldots$

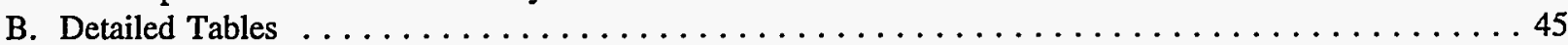

C. U.S. Climate Zones and Census Regions and Divisions Maps $\ldots \ldots \ldots \ldots \ldots \ldots \ldots \ldots \ldots \ldots$

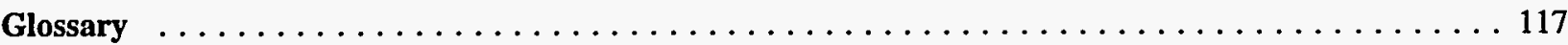

\section{Tables}

B1. Consumption of All Major Fuels by End Use, $1989 \ldots \ldots \ldots \ldots \ldots \ldots \ldots \ldots \ldots \ldots \ldots$

B2. Energy End-Use Intensities for All Major Fuels, $1989 \ldots \ldots \ldots \ldots \ldots \ldots \ldots \ldots \ldots \ldots \ldots$

B3. End-Use Consumption Percentages for All Major Fuels, $1989 \ldots \ldots \ldots \ldots \ldots \ldots \ldots \ldots \ldots \ldots$

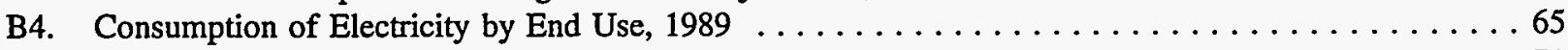

B5. Energy End-Use Intensities for Electricity, $1989 \ldots \ldots \ldots \ldots \ldots \ldots \ldots \ldots \ldots \ldots \ldots \ldots$

B6. End-Use Consumption Percentages for Electricity, $1989 \ldots \ldots \ldots \ldots \ldots \ldots \ldots \ldots \ldots \ldots \ldots$

B7. Consumption of Natural Gas by End Use, $1989 \ldots \ldots \ldots \ldots \ldots \ldots \ldots \ldots \ldots \ldots \ldots \ldots$

B8. Energy End-Use Intensities for Natural Gas, $1989 \ldots \ldots \ldots \ldots \ldots \ldots \ldots \ldots \ldots \ldots$

B9. End-Use Consumption Percentages for Natural Gas, $1989 \ldots \ldots \ldots \ldots \ldots \ldots \ldots \ldots \ldots \ldots$

B10. Consumption of Fuel Oil by End Use, $1989 \ldots \ldots \ldots \ldots \ldots \ldots \ldots \ldots \ldots \ldots \ldots \ldots$

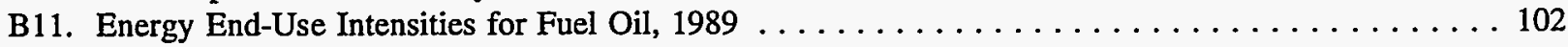


B12. Consumption of District Heat by End Use, $1989 \ldots \ldots \ldots \ldots \ldots \ldots \ldots \ldots \ldots \ldots \ldots \ldots$

B13. Energy End-Use Intensities for District Heat, $1989 \ldots \ldots \ldots \ldots \ldots \ldots \ldots \ldots \ldots \ldots \ldots \ldots$

\section{Figures}

1. Energy Consumption in U.S. Commercial Buildings, by Energy Source, $1989 \ldots \ldots \ldots \ldots \ldots \ldots \ldots 2$

2. Energy Consumption in U.S. Commercial Buildings, by Principal Building Activity, $1989 \ldots \ldots \ldots \ldots 3$

3. Energy Consumption in U.S. Commercial Buildings, by End Use, $1989 \ldots \ldots \ldots \ldots \ldots \ldots \ldots \ldots$

4. Shares of Consumption for Electricity and Natural Gas, by End Use, $1989 \ldots \ldots \ldots \ldots \ldots \ldots$

5. Shares of End-Use Site Energy Consumption, Primary Energy Consumption, and Expenditures,

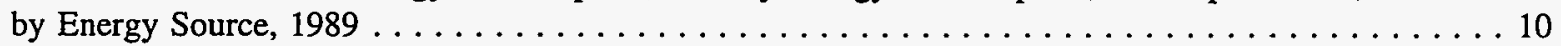

6. Energy End Uses Ranked by Site Energy Consumption and by Energy Expenditures, $1989 \ldots \ldots \ldots \ldots 11$

7. Energy Intensities for Commercial Buildings, $1989 \ldots \ldots \ldots \ldots \ldots \ldots \ldots \ldots \ldots \ldots \ldots \ldots \ldots$

8. Energy Intensities for Commercial Buildings, by Climate Zone, $1989 \ldots \ldots \ldots \ldots \ldots \ldots \ldots \ldots$

9. End-Use Intensity Profiles, by Principal Building Activity, $1989 \ldots \ldots \ldots \ldots \ldots \ldots \ldots \ldots \ldots \ldots$

10. Percent of Energy Supplied for Space Heating, by Energy Source, 1989 . . . . . . . . . . . . . . . 18

11. Percent of Heated Floorspace Served by Each Main Space-Heating Energy Source and

Percent of Space-Heating Consumption, by Year Constructed, 1989 . . . . . . . . . . . . . . 19

12. Comparison of Main Space-Heating Intensities for Natural Gas and Electricity,

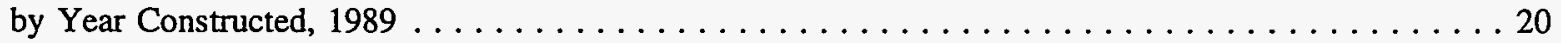

13. Comparison of Main Space-Heating Intensities for Natural Gas and Electricity,

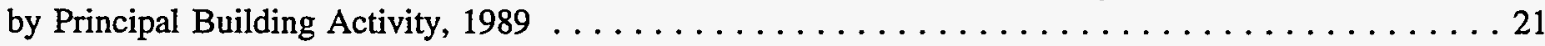

14. Heating Intensities in Buildings Where Natural Gas Was Main Heating Fuel, by Year Constructed

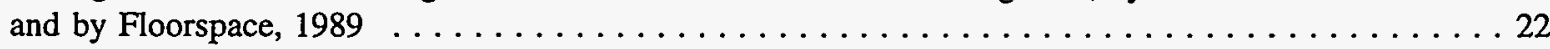

15. Heating Intensities in Buildings Where Natural Gas Was Main Heating Fuel, by Weekly Operating Hours and by Climate Zone, $1989 \ldots \ldots \ldots \ldots \ldots \ldots \ldots \ldots \ldots$

16. Heating Intensities in Buildings Where Electricity Was Main Heating Fuel, by Year Constructed

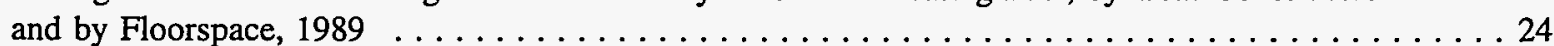

17. Electric Cooling and Ventilation Intensities, by Year Constructed, $1989 \ldots \ldots \ldots \ldots \ldots \ldots \ldots \ldots$

18. Electric Cooling and Ventilation Intensities, by Floorspace, $1989 \ldots \ldots \ldots \ldots \ldots \ldots \ldots \ldots \ldots$

19. Electric Cooling and Ventilation Intensities, by Principal Building Activity, $1989 \ldots \ldots \ldots \ldots \ldots$

20. Electric Cooling and Ventilation Intensities, by Climate Zone, $1989 \ldots \ldots \ldots \ldots \ldots \ldots \ldots \ldots$

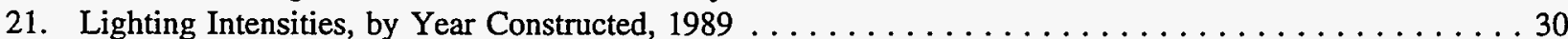

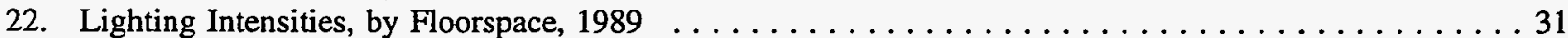

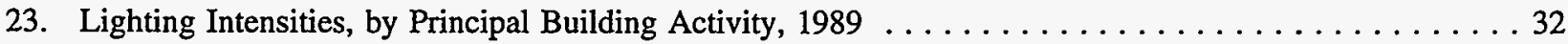

24. Intensities for Natural Gas Water Heating and Cooking and for Electric Refrigeration,

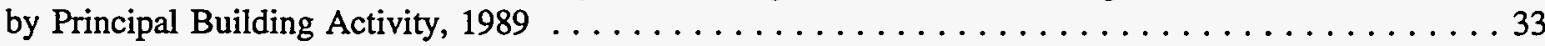

25. Electric Intensities for Office Equipment, by Principal Building Activity and

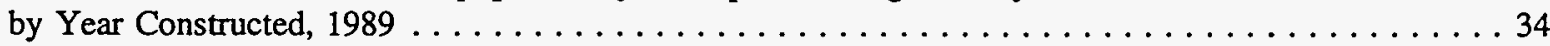

26. Actual and Hypothetical Consumption of Natural Gas for Main Space Heating $\ldots \ldots \ldots \ldots \ldots 36$

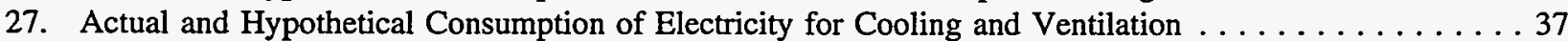

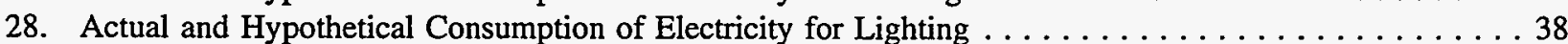

29. Actual and Hypothetical Consumption of Electricity for Office Equipment $\ldots \ldots \ldots \ldots \ldots \ldots \ldots$

30. Summary of Changes for Selected End-Use Energy Consumption and Expenditures that Would Occur If All Buildings Consumed at the Rate of 1980 's Buildings $\ldots \ldots \ldots \ldots \ldots \ldots \ldots \ldots \ldots \ldots$ 


\section{Executive Summary}

The demand for energy in U.S. stores, offices, schools, hospitals, and other commercial buildings has been increasing. This report examines energy intensities in commercial buildings for nine end uses: space heating, cooling, ventilation, lighting, water heating, cooking, refrigeration, office equipment, and "other." The objective of this analysis was to increase understanding of how energy is used in commercial buildings and to identify targets for greater energy efficiency which could moderate future growth in demand.

The source of data for the analysis is the 1989 Commercial Buildings Energy Consumption Survey (CBECS), which collected detailed data on energy-related characteristics and energy consumption for a nationally representative sample of approximately 6,000 commercial buildings. The analysis used 1989 CBECS data because the 1992 CBECS data were not yet available at the time the study was initiated. The CBECS data were fed into the Facility Energy Decision Screening (FEDS) system, a building energy simulation program developed by the U.S. Department of Energy's Pacific Northwest Laboratory, to derive engineering estimates of end-use consumption for each building in the sample. The FEDS estimates were then statistically adjusted to match the total energy consumption for each building.

This is the Energy Information Administration's (EIA) first report on energy end-use consumption in commercial buildings. This report is part of an effort to address customer requests for more information on how energy is used in buildings, which was an overall theme of the 1992 user needs study. (See User-Needs Study for the 1992 Commercial Buildings Energy Consumption Survey, DOE/EIA-0555(92)/4, Washington, DC, September 1992.) The end-use data presented in this report were not available for publication in Commercial Buildings Energy Consumption and Expenditures 1989 (DOE/ELA-0318(89), Washington, DC, April 1992). However, subsequent reports on end-use energy consumption will be part of the Commercial Buildings Energy Consumption and Expenditures series, beginning with a 1992 data report to be published in early 1995 .

Since EIA is publishing commercial energy end-use estimates for the first time, readers are urged to provide comments and suggestions for the improvement of 1992 and future end-use energy estimates.

\section{Overview of Energy Consumption in Commercial Buildings}

In spite of the gains in energy efficiency made since the 1970's, economic expansion and increases in energyconsuming services during the 1980's caused a net increase in energy consumption in the commercial sector. Energy demand in the commercial sector grew during the 1980's by 1.0 percent a year, second only to the transportation sector's rate of 1.4 percent a year. In contrast, energy demand in both the residential and industrial sectors declined during the 1980's. Commercial buildings in 1989 consumed almost 6 quadrillion Btu of energy for end uses and ran up an energy bill of over $\$ 70$ billion.

By type of building, the three largest energy users were (a) office buildings (1.2 quadrillion Btu); (b) department stores, drugstores, gasoline stations, post offices, and other mercantile and service buildings (1.0 quadrillion Btu); and (c) education buildings ( 0.7 trillion Btu).

Of the various end uses, space heating accounted for the largest share of consumption ( 35 percent), followed by lighting (18 percent), water heating ( 9 percent), office equipment ( 7 percent), cooling (5 percent), ventilation ( 5 percent), cooking ( 5 percent), and refrigeration ( 3 percent).

Of the four major energy sources (electricity, natural gas, fuel oil, and district heat) consumed in commercial buildings in 1989, electricity accounted for almost 50 percent of all the energy delivered to commercial sites, while natural gas accounted for 36 percent of site consumption. Natural gas space heating and electric lighting were the 
two largest consumers of site energy. However, the amount spent on electric lighting was more than double the amount spent on natural gas for all end uses, due to the higher price of electricity compared with the price of natural gas.

An "energy intensity" is the ratio of energy consumption to a measure of the demand for energy services. A common measure of energy intensity is the ratio of the amount of energy consumed for the building as a whole or for a particular end use to the square footage of a building's floorspace. More precise measures of energy intensity can account for such factors as building operating hours or weather conditions. Intensity ratios allow buildings to be compared in terms of energy consumption, even though they are of different sizes, are located in different climates, and have different operating hours.

Analysis of energy intensities for the buildings in the 1989 CBECS showed that:

- The highest energy intensities per square foot were found in buildings constructed in the 1960 's, with buildings constructed in the 1980's continuing a trend towards lower intensities.

- Buildings in the coolest climates had the highest energy intensities per square foot because of their greater demand for space heating, despite their lower demand for cooling.

- Each type of commercial building had a different end-use intensity profile, with no two end-use profiles being alike. Some end uses, such as heating, cooling, ventilation, and lighting, were found, to some degree, in all types of buildings. However, other end uses reflected the special activities performed within particular types of buildings.

\section{Space-Conditioning Intensities}

Space conditioning--heating, cooling, and ventilation--accounted for 45 percent of all energy consumed in commercial buildings in 1989. Natural gas was the dominant energy source for space heating in commercial buildings in 1989, providing 63 percent of all the energy consumed for space heating.

- Whether electricity is measured by site energy (consumed by the end user) or by primary energy (consumed at the generating plant), natural gas provided a much higher percentage of the space heating energy consumed in buildings constructed during the 1980's than electricity did, even though the amount of floorspace heated by the two energy sources was roughly equal.

- Buildings constructed in the 1970's had the highest cooling intensities, defined as the ratio of energy used for cooling to the product of the cooled square footage, the annual building operating hours, and the average daily cooling degree-days (CDD's).

- Larger buildings had the highest ventilation intensities, defined as the ratio of energy used for ventilation to the product of the square footage and the annual building operating hours.

\section{Intensities of Other End Uses}

Other end uses--lighting, water heating, cooking, refrigeration, office equipment, and miscellaneous uses--accounted for 55 percent of all energy consumed in commercial buildings in 1989. Lighting intensities were defined as the ratio of energy used for lighting to the product of the lighted square footage and the annual building operating hours. Intensities for the remaining end uses were defined as the ratio between the energy used and the product of square footage and the annual operating hours. 
- Office buildings had the highest intensities for lighting and for office equipment.

- Buildings constructed during the 1970's and 1980's had the highest lighting intensities, while the oldest buildings had the lowest lighting intensities.

- Food service and health care buildings had the highest water-heating intensities per square foot--more than five times the average for all buildings.

- Food sales and food service buildings had the highest energy intensities for cooking and refrigeration.

\section{Targets for Reducing Energy Intensities}

The method used to determine targets for reducing energy intensities was to extrapolate the energy consumption patterns of the 1980's buildings to the entire commercial building stock. Intensities were based upon the entire building stock, not just buildings using a particular fuel for a given end use. This method of extrapolation reflected both the level of penetration and the efficiencies of 1980's technologies in computing hypothetical consumption levels for the total building stock.

- If all commercial buildings used natural gas for heating with the same intensity as did buildings constructed in the 1980's, the total consumption of natural gas for space heating would fall 201 trillion Btu, or 17 percent.

- If all buildings used electricity for cooling with the same intensity as did buildings constructed in the 1980's, the total consumption of electricity for cooling would drop by 9 percent $(23$ trillion Btu). However, the consumption of electricity for ventilation would rise by 5 percent (14 trillion Btu), for a net reduction of 9 trillion Btu, or 2 percent.

The analysis showed that lighting and office equipment presented especially worthwhile opportunities for moderating future growth in energy demand by increasing energy efficiency. Although energy efficiencies may have improved during the 1980's, the demand for these two energy services, especially for office equipment, also increased dramatically.

- If all commercial buildings had the same lighting intensity as buildings constructed in the 1980's, consumption of electricity for lighting would increase 9 percent ( 94 trillion Btu).

- If all buildings used energy for office equipment with the same intensity as 1980's buildings, consumption of electricity for office equipment would increase by 26 percent ( 99 trillion Btu). 


\section{Section 1. Introduction}

The demand for energy has been increasing in U.S. stores, offices, schools, hospitals, and other commercial buildings. To find out where the best opportunities lie for improving energy efficiency, this report looks at the intensities of energy use in commercial buildings in 1989, the latest year for which energy consumption figures for commercial buildings are available.

This report is the first published by the Energy Information Administration (EIA) on the topic of energy end-use consumption in commercial buildings. The study of end-use energy consumption is important for the understanding of how and why energy is used. Conceptually, the end-uses of energy provide an intermediate level of analysis, below the consuming unit (the commercial building) but above the actual equipment used to perform the end use.

This report is part of an effort to address requests from customers for more information on how energy is used in buildings--an overall theme of the 1992 user needs study. (See User-Needs Study for the 1992 Commercial Buildings Energy Consumption Survey, DOE/EIA-0555(92)/4, Washington, DC, September 1992.) Subsequent reports on enduse energy consumption will be part of the Commercial Buildings Energy Consumption and Expenditures series, beginning with a 1992 data report to be published in early 1995. It is hoped that each successive report will increase understanding of how energy is being used in commercial buildings and, consequently, of where the best opportunities for improving energy efficiency lie.

\section{Report Outline}

This report has five sections. Section 1 gives background information on energy consumption in commercial buildings and introduces energy end-use intensities. Section 2 presents an overview of energy consumption and energy intensities in commercial buildings. Section 3 is devoted to the largest end use: space conditioning--heating, cooling, and ventilation. Section 4 deals with all other end uses--lighting, water heating, cooking, refrigeration, and office equipment. The concluding section, Section 5, discusses potential targets for reducing end-use intensities in commercial buildings.

The main body of the report is followed by three appendices and a glossary. Appendix A discusses the methodology for developing the end-use intensity estimates. Appendix B consists of 13 tables presenting detailed energy end-use consumption data. Appendix $\mathrm{C}$ contains maps of U.S. climate zones and Census regions and divisions. The report concludes with a Glossary, which should be consulted for explanations of terms used in this report.

\section{Trends in Energy Consumption in Commercial Buildings}

In the 1960's, when energy was cheap and the earth's resources seemed inexhaustible, building designers seldom discussed the conservation of energy during the design process. They saw little reason for making a building in Maine look different from one in Florida, and they favored all-glass buildings in all regions. They considered the climate in which the building was located to be largely irrelevant.

Then came the oil supply disruptions of 1973 and 1979, which caused the price of oil to skyrocket and triggered fears about U.S. energy security. At the same time, reports on how fossil fuel emissions were damaging the air, water, plant life and wildlife made Americans aware of the need to protect the environment.

As a result, energy conservation became a major concern in the 1980's. New commercial buildings built in that decade were designed for their specific climates. They were well-insulated, with multipane and reflective windows, more efficient space conditioning equipment, and better lighting systems. Furthermore, many older buildings were retrofitted to improve their energy efficiency. 
Figure 1. Energy Consumption in U.S. Commercial Buildings, by Energy Source, 1989

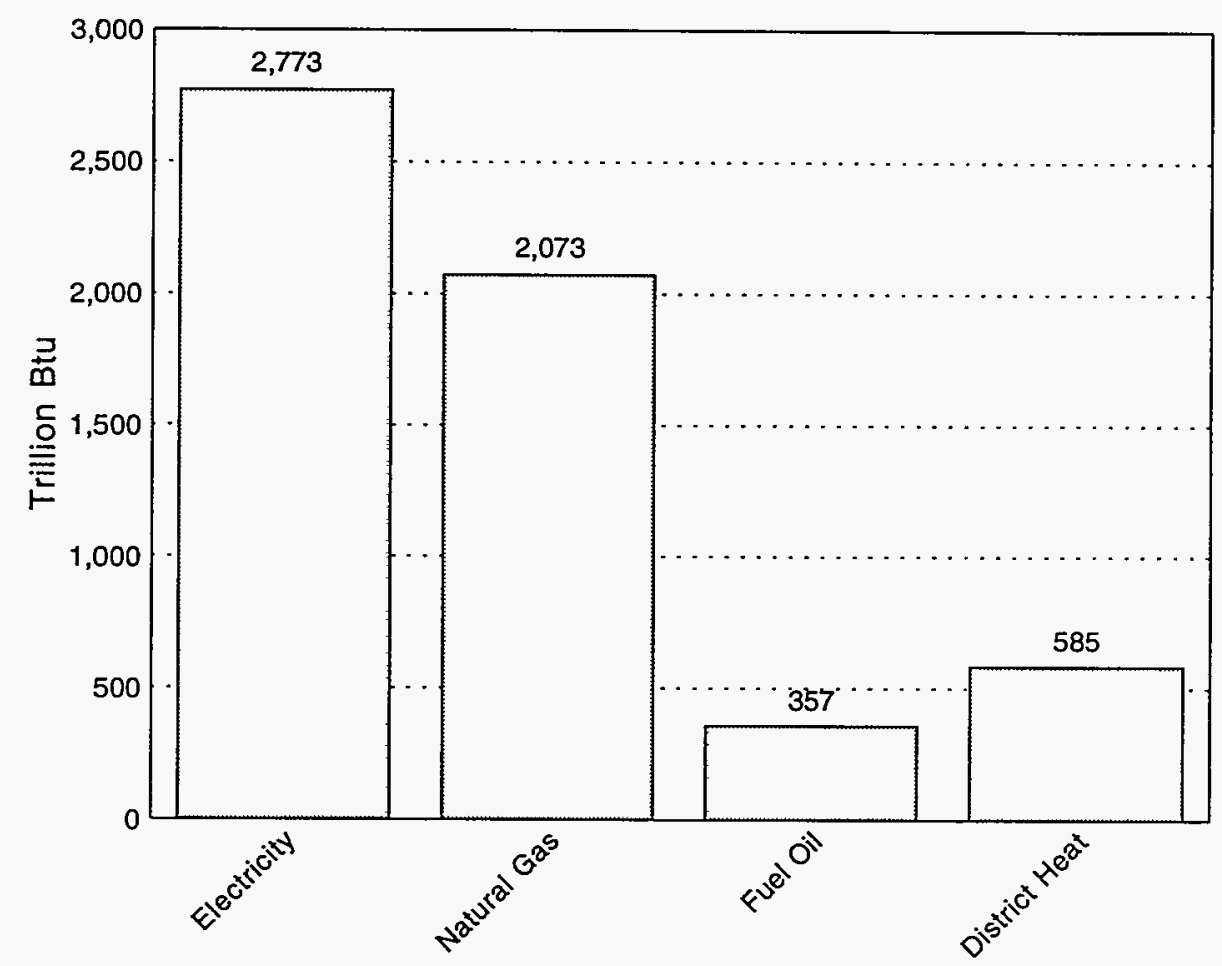

Source: Energy Information Administration, Office of Energy Markets and End Use, Forms ElA-871A through F of the 1989 Commercial Buildings Energy Consumption Survey.

In spite of the gains in energy efficiency made since the 1970's, an expanding economy and increases in energyconsuming services during the 1980's caused a net increase in energy consumption in the commercial sector. This demand was largely due to more computers and other office equipment, better lighting, and more comfortable heating, cooling, and ventilation. In the 1980's, energy demand in the commercial sector grew by 1.0 percent a year, second only to the transportation sector's rate of 1.4 percent a year. In contrast, energy demand in both the residential and industrial sectors declined during the 1980's.

In 1989, U.S. commercial buildings used 5.8 quadrillion Btu of energy, the equivalent to 260 million short tons of coal, 5.6 trillion cubic feet of dry natural gas, 1.0 billion barrels of crude oil, or 128 days of petroleum imports.

- Figure 1 shows that commercial buildings used 813 billion kilowatthours $(2,773$ trillion Btu) of electricity, equivalent to 478 million barrels of crude oil.

- Commercial buildings used 2.0 trillion cubic feet (2,073 trillion Btu) of natural gas, equivalent to 357 million barrels of crude oil.

- Commercial buildings used 2.55 billion gallons ( 357 trillion Btu) of fuel oil, equivalent to 61 million barrels of crude oil.

- Commercial buildings used 585 trillion Btu of district heat (steam and hot water delivered to a building from a central plant or utility), equivalent to 569 billion cubic feet of natural gas or 4.2 billion gallons of fuel oil. 
Figure 2. Energy Consumption in U.S. Commercial Buildings, by Principal Building Activity, 1989

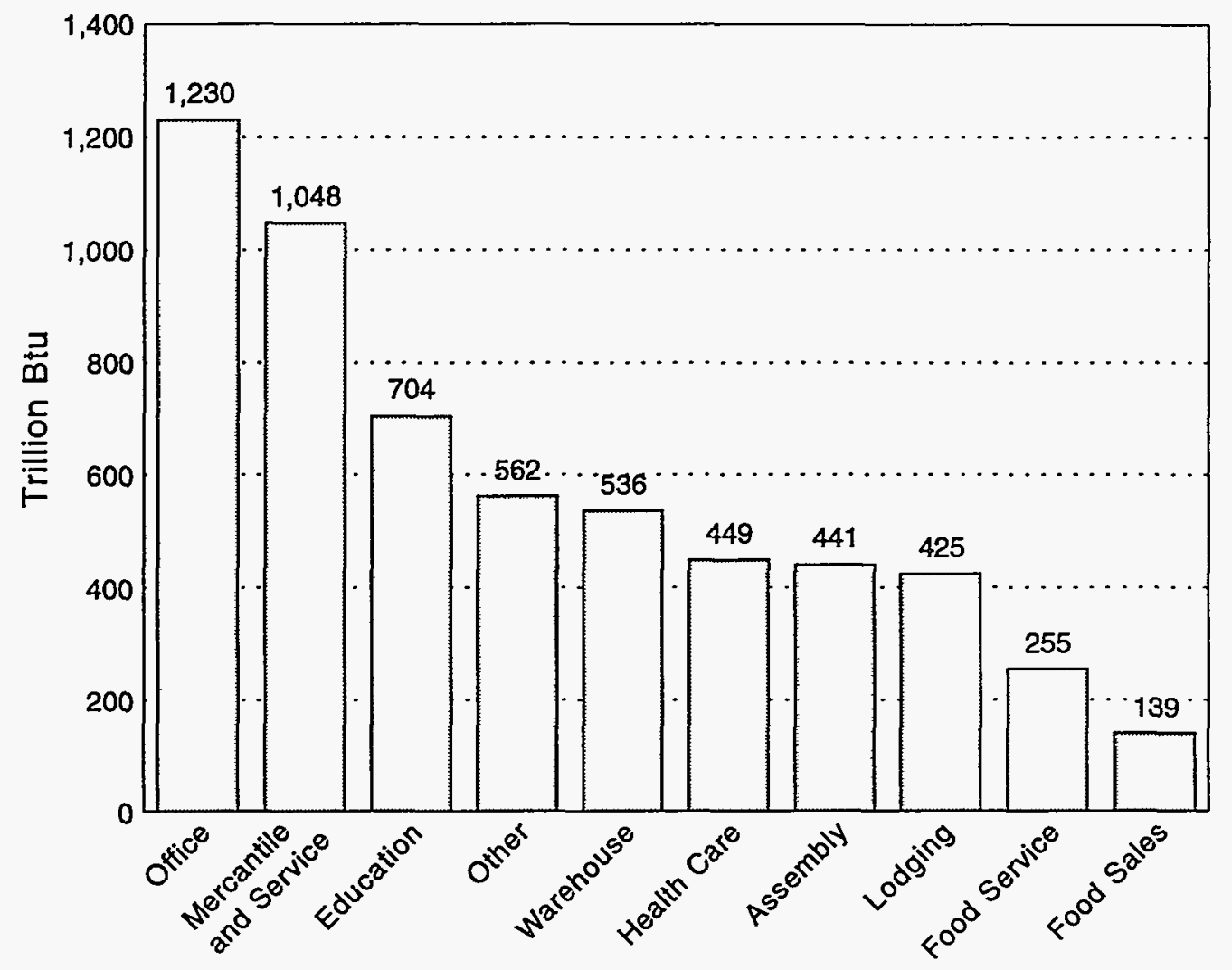

Source: Energy Information Administration, Office of Energy Markets and End Use, Forms ElA-871A through F of the 1989 Commercial Buildings Energy Consumption Survey.

- Figure 2 shows that the three largest energy users were: (a) office buildings (1,230 trillion Btu); (b) department stores, drugstores, automotive dealers, gasoline stations, laundries, post offices and other mercantile and service buildings (1,048 trillion Btu); and (c) education buildings (704 trillion Btu).

- The next largest energy users were: (a) warehouses (536 trillion Btu); (b) hospitals and other health care buildings (449 trillion Btu); (c) concert halls, night clubs, theaters, bowling alleys, indoor pools, skating rinks, auditoriums, convention halls, stadiums, and other buildings used for assembly (441 trillion Btu); and (d) hotels, motels, convents, dormitories, and other buildings used for lodging (425 trillion Btu).

- The smallest energy users were: (a) restaurants and other food service buildings ( 255 trillion Btu), and (b) supermarkets, grocery stories, bakeries, and other buildings used for food sales (139 trillion Btu).

- Laboratories, parking garages, public order and safety buildings, vacant buildings and all other commercial buildings not included in above categories used 562 trillion Btu. 


\section{Energy Intensities for Commercial End Uses}

"Energy intensity" is the term used to express the ratio of energy consumption to a measure of the demand for energy services. A common measure of energy intensity is the ratio of the amount of energy consumed for the building as a whole or for a particular end use to the square footage of a building's floorspace. This is the measure used throughout Appendix B, "Detailed Tables." However, a more precise measure would consider the building's operating hours and climate. Taking these factors into account allows the energy intensities of buildings to be compared, even though the buildings are of different sizes, are located in different climates, and have different operating hours.

In this report, energy intensities are often measured by taking into account operating hours or climate. For example:

- Space-heating intensity is expressed as the ratio of the energy used for space heating to the product of (1) the number of square feet of heated floorspace in a building, (2) the building's annual operating hours, and (3) the average daily number of "heating degree-days" (HDD's). The HDD's for a single day are the difference between 65 degrees Fahrenheit and the average temperature if the average temperature is below 65 degrees Fahrenheit, and is zero otherwise. Obviously, the more HDD's a building experiences the higher will be its demand for space heating.

- Cooling intensity is expressed in the same way as space-heating intensity, except that "cooling degree-days" (CDD's) are used instead of HDD's. The CDD's for a single day are the difference between 65 degrees Fahrenheit and the average temperature if the average temperature is above 65 degrees Fahrenheit, and is zero otherwise. Again, the more CDD's a building experiences the higher will be its demand for cooling.

- Ventilation intensity is expressed as the ratio of the energy used for ventilation to the product of the square footage of the building and the annual building operating hours. Since ventilation is used for both heating and cooling, as well as for circulating air within a building, HDD's and CDD's are irrelevant.

- Lighting intensity is expressed as the ratio of the energy used for lighting to the product of the square footage of lighted floorspace and the annual building operating hours.

- All other intensities (water heating, cooking, refrigeration, office equipment, and "other") are expressed only as the ratio of the energy used for the particular end use to the square footage of building floorspace.

\section{Data Sources and Methodology}

The estimates of end-use energy intensities presented in this report were based on data from the 1989 Commercial Buildings Energy Consumption Survey (CBECS) in conjunction with end-use estimates modelled by the Facility Energy Decision Screening (FEDS) system.

The CBECS is a nationally representative probability sample of commercial buildings. For purposes of this survey, a commercial building is defined as one whose principal activity is not residential or industrial. The survey covers all commercial buildings over 1,000 square feet. For each of the roughly 6,000 buildings in the sample, the CBECS collects data on (1) energy-related characteristics of the building through personal interviews with the buildings' owners or managers and (2) total energy consumption for all end uses from billing data provided by the buildings'

energy suppliers. The 1989 CBECS, which provides data for that calendar year, was used for the present analysis because this survey was the latest one available at the time the study was initiated. It is anticipated that the report containing estimates for 1992 will be published in early 1995. 
The separate end-use consumption estimates were derived for each sampled building by using the FEDS system, a building energy simulation program developed at the Pacific Northwest Laboratory for the U.S. Department of Energy's Federal Energy Management Program and the U.S. Army Construction Engineering Research Laboratory. The FEDS engineering model was originally designed to assess the potential for energy retrofits at large federal installations. The FEDS was used to calculate the initial engineering end-use estimates for each building in the 1989 CBECS sample. These engineering estimates were then statistically adjusted to match the total energy consumption for each building.

The method for identifying targets for reducing energy intensities relied on the extrapolation of the energy consumption patterns of the 1980's buildings to the entire commercial building stock. This method highlights the ways in which new construction differs from the rest of the building stock. Thus, the end uses with significantly higher energy consumption would make the best targets for energy efficiency measures.

The method, performed for five major end uses (natural gas space heating and electric cooling, ventilation, lighting, and office equipment use), had two parts.

- First, the energy intensities of 1980 's buildings were calculated by using the more precise measures of energy intensities. Energy intensities were calculated by principal building activity categories. For the five most numerous types of buildings in the CBECS sample (education, health care, mercantile, office, and warehouse), the buildings were divided into two groups: (1) small buildings, having 50,000 square feet of floorspace or less, and (2) large buildings, having over 50,000 square feet of floorspace.

- Second, estimates were made of the energy intensities and consumption for the five end uses, assuming that all buildings had the same end-use intensities as those of 1980's buildings.

The end uses that showed the greatest growth in consumption were identified as targets for energy efficiency measures.

For further information on sources and methodology, see Appendix A, "The Development of End-Use Intensity Estimates." 
. 


\section{Section 2. Energy Use in Commercial Buildings}

The purpose of this section is to provide an overview of how energy was used in commercial buildings. Focusing on 1989 buildings, the section shows energy consumption by energy source (electricity, natural gas, fuel oil, and district heat), by energy end use (space heating, cooling, ventilation, water heating, lighting, cooking, refrigeration, office equipment, and other), and by various building characteristics (floorspace, year constructed, weekly operating hours, climate zone, and building activity).

Key findings of this section include:

- Of all the various end uses, space heating accounted for the largest share of consumption ( 35 percent), followed by lighting ( 18 percent), water heating ( 9 percent), office equipment ( 7 percent), cooling ( 5 percent), ventilation ( 5 percent), cooking ( 5 percent), and refrigeration ( 3 percent) (Figure 3$)$.

- Natural gas consumption was dominated by consumption for space heating, accounting for 61 percent of the natural gas consumed (Figure 4).

- Electricity accounted for almost 50 percent of all the energy delivered to commercial sites, while natural gas accounted for 36 percent of site consumption (Figure 5).

- Electricity accounted for over 70 percent of the primary energy consumption (the amount of energy consumed at commercial sites plus the amount of energy lost in producing and transmitting electricity and district heat) at commercial buildings, while natural gas accounted for only 18 percent of primary energy consumption (Figure 5).

- Electricity accounted for over 80 percent of the energy expenditures for commercial buildings, while natural gas accounted for only 13 percent (Figure 5).

- Natural gas space heating and electric lighting were the two largest consumers of site energy (Figure 6).

- Electric lighting accounted for the largest portion of energy expenditures in commercial buildings (Figure 6).

- Defining a building's energy intensity as the ratio of energy consumption to floorspace, the highest intensities were found in buildings constructed in the 1960's. Buildings constructed in the 1980's showed lower intensities (Figure 7).

- Buildings located in the coldest climate had the highest energy intensity because of the demand for space heating. Conversely, the buildings located in the warmest climate, had the lowest energy intensity because of the small demand for space heating, despite their greater need for cooling (Figure 8).

- The different types of commercial buildings had distinctive energy-use profiles (Figure 9). 


\section{Energy Consumption by End Use}

Figure 3. Energy Consumption in U.S. Commercial Buildings, by End Use, 1989

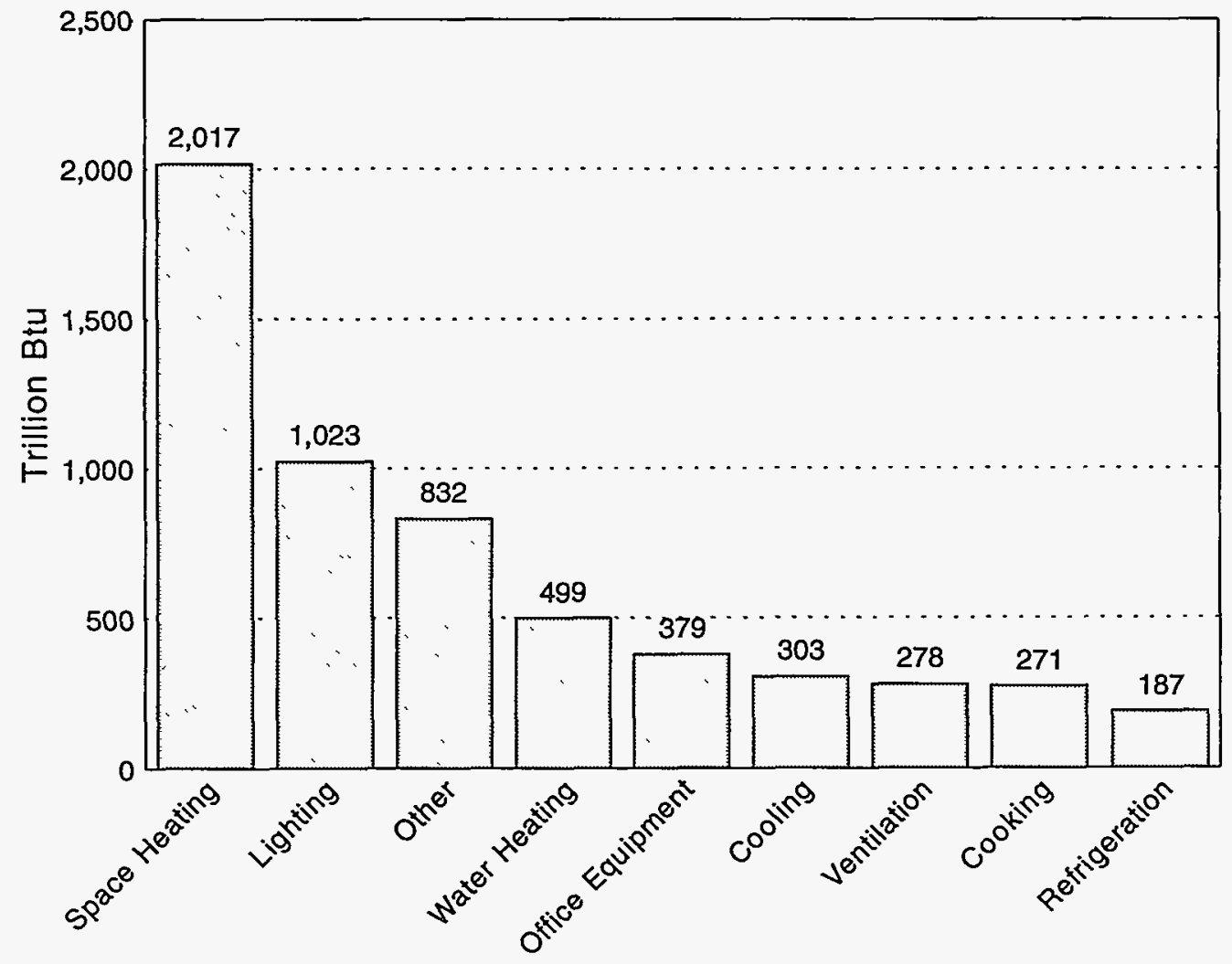

Sources: Energy Information Administration, Office of Energy Markets and End Use, Forms EIA-871A through F of the 1989 Commercial Buildings Energy Consumption Survey and statistically adjusted engineering end-use estimates.

- Figure 3 shows that of all the energy used for the various end uses in commercial buildings in 1989 , the largest share was for space heating ( 35 percent), followed by lighting (18 percent), water heating ( 9 percent), office equipment ( 7 percent), cooling ( 5 percent), ventilation ( 5 percent), cooking ( 5 percent), and refrigeration ( 3 percent).

- Several other end uses, which have been grouped in the category called "other," used 14 percent of all energy used in buildings. Included in this category is energy consumed for such uses as elevators, medical and other laboratory equipment, and miscellaneous electrical appliances. 
a. Electricity

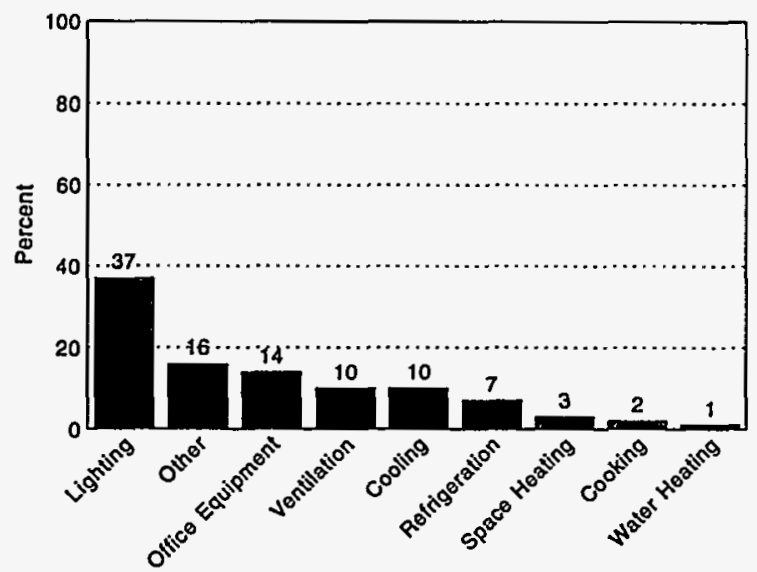

b. Natural Gas

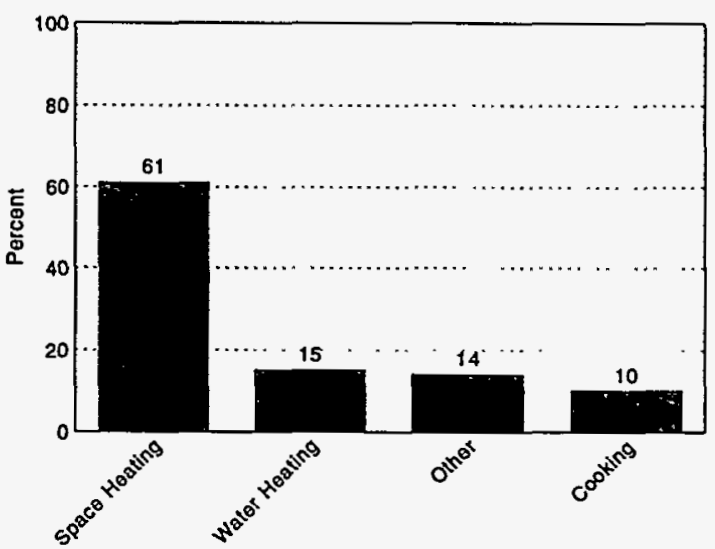

Sources: Energy Information Administration, Office of Energy Markets and End Use, Forms ElA-871A through F of the 1989 Commercial Buildings Energy Consumption Survey and statistically adjusted engineering end-use estimates.

- The two main energy sources consumed in commercial buildings in 1989 were electricity (2.8 quadrillion Btu) and natural gas (2.1 quadrillion Btu) (Figure 1).

- Figure 4 shows that electricity usage was spread among all nine end uses, while natural gas usage was more restricted to end uses involving heating (space heating, water heating, and cooking).

- Lighting was the largest single use of electricity, accounting for 37 percent of the electricity consumed.

- Natural gas consumption was dominated by space heating, accounting for 61 percent of the natural gas consumed. 
Figure 5. Shares of End-Use Site Energy Consumption, Primary Energy Consumption, and Expenditures, by Energy Source, 1989

a. Site Energy Consumption

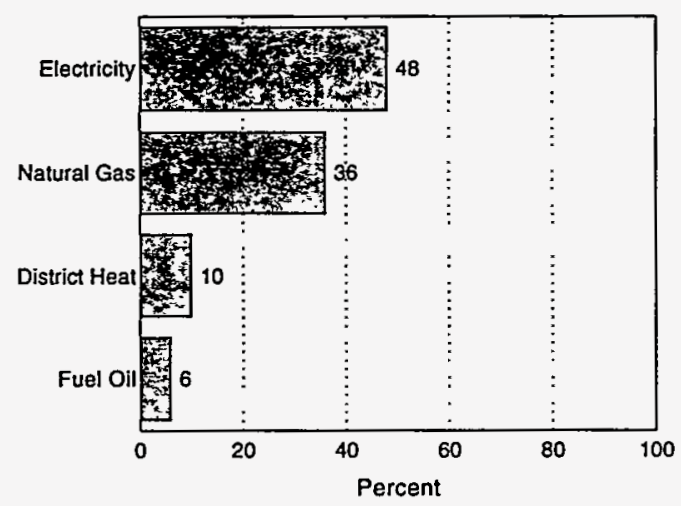

b. Primary Energy Consumption

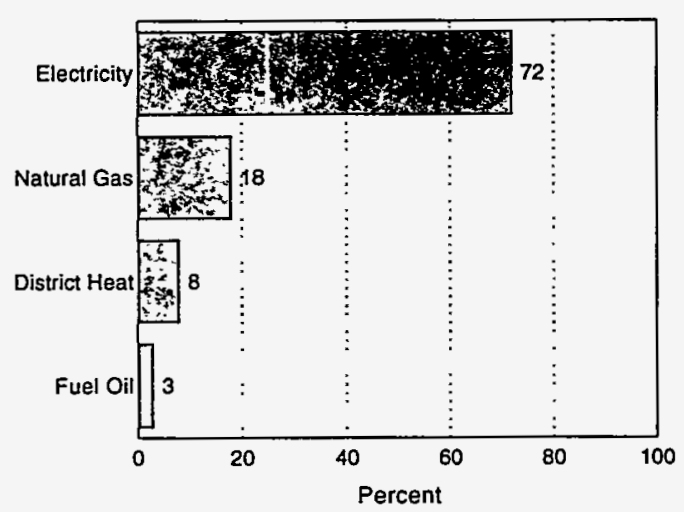

c. Energy Expenditures

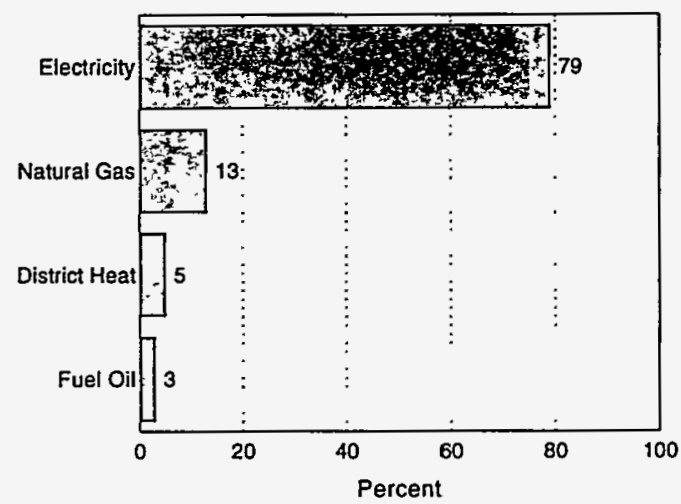

Source: Energy Information Administration, Office of Energy Markets and End Use, Forms ElA-871A through F of the 1989 Commercial Buildings Energy Consumption Survey.

- Energy consumption can be measured by (1) the amount of energy delivered to a site (site consumption), (2) the amount of energy delivered to a site plus the amount of energy used to produce and transmit the electricity or district heat (primary energy consumption), or (3) the amount of money paid for the energy consumed at a site (energy expenditures).

- Figure 5 shows that electricity accounted for almost 50 percent of the site energy consumption, over 70 percent of the primary energy consumption, and almost 80 percent of the energy expenditures in commercial buildings.

- Natural gas, which accounted for 36 percent of site energy consumption, accounted for only 13 percent of energy expenditures, reflecting the lower price of natural gas in 1989 as with prices of other energy sources.

- Electricity, which in 1989 averaged $\$ 20.17$ per million Btu of site energy (6.9 cents per kilowatthour), was the most expensive energy source. (Natural gas averaged $\$ 4.44$ per million Btu; fuel oil, $\$ 5.10$ per million Btu; and district heat, $\$ 6.59$ per million Btu.)

- At a price of $\$ 6.72$ per million Btu of primary energy, electricity was still the most expensive energy source, but not by a wide margin. (The primary prices of natural gas and fuel oil were the same as the site prices; the primary price of district heat was $\$ 4.40$ per million Btu.) 
Figure 6. Energy End Uses Ranked by Site Energy Consumption and by Energy Expenditures, 1989

a. Site Energy Consumption

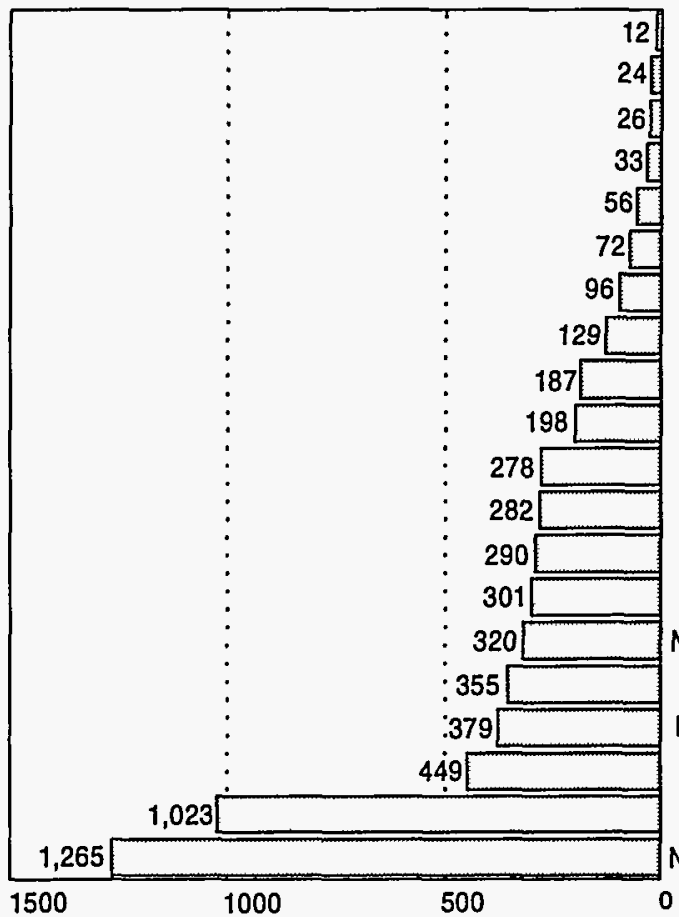

b. Energy Expenditures

Sources: Energy Information Administration, Office of Energy Markets and End Use, Forms EIA-871A through F of the 1989 Commercial Buildings Energy Consumption Survey and statistically adjusted engineering end-use estimates.

- Figure 6 shows that rankings of the top energy end uses differed markedly, depending on the criterion used (site consumption or expenditures). Rankings by primary energy were very similar to rankings by expenditures, due to the relatively narrow range of primary prices among the energy sources.

- Natural gas space heating and electric lighting were the two largest consumers of site energy. Each accounted for over 1 quadrillion Btu of energy consumption, out of a total site energy consumption of nearly 6 quadrillion Btu.

- Energy end-use expenditures can be approximated by multiplying the average fuel price by the amount of energy consumed for an end use.

- Electric lighting accounted for the largest portion of energy end-use expenditures in commercial buildings.

- The amount spent on electric lighting was more than double the amount spent on natural gas for all uses, and was considerably more than the amount spent for natural gas, fuel oil, and district heat combined. 


\section{Major Influences on Energy Intensities}

Figure 7. Energy Intensities for Commercial Buildings, 1989

a. Floorspace

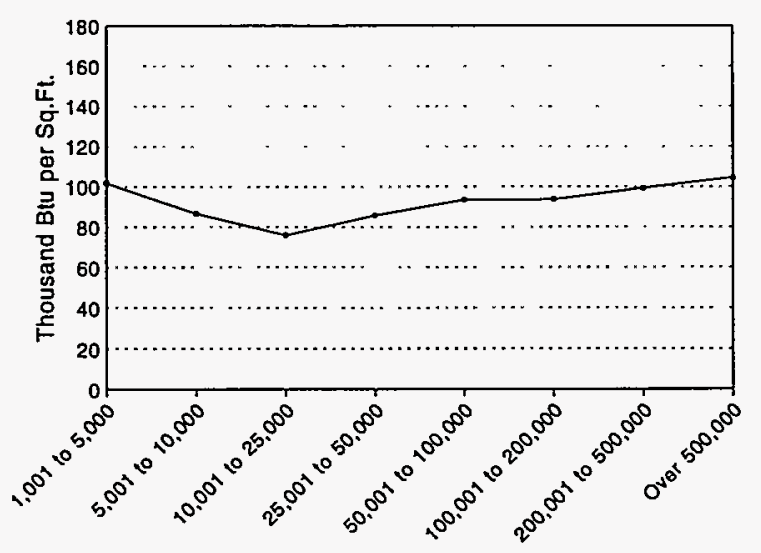

b. Year Constructed

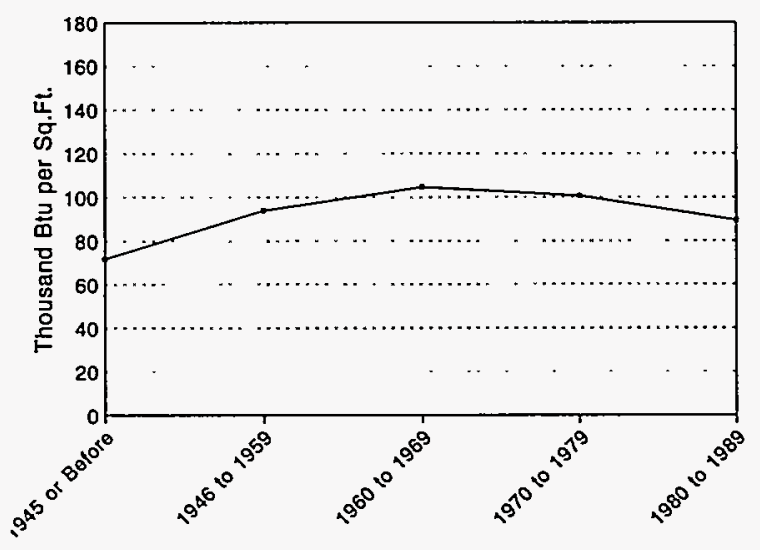

c. Weekly Operating Hours

Source: Energy Information Administration, Office of Energy Markets and End Use, Forms ElA-871A through F of the 1989 Commercial Buildings Energy Consumption Survey.

- "Energy intensity" is the ratio of energy consumption to a measure of the demand for energy services (such as building floorspace or weekly operating hours). Energy intensities facilitate the comparison of energy use across different kinds of buildings.

- Figure 7 shows that intensities per square foot were relatively flat across floorspace categories, indicating that the normalization by square footage largely achieved its aim.

- In general, the highest energy intensities were found in buildings constructed in the 1960's. Buildings constructed in the 1980's continued a trend towards lower intensities.

- In general, the longer the weekly operating hours, the higher the building's energy intensity per square foot. 

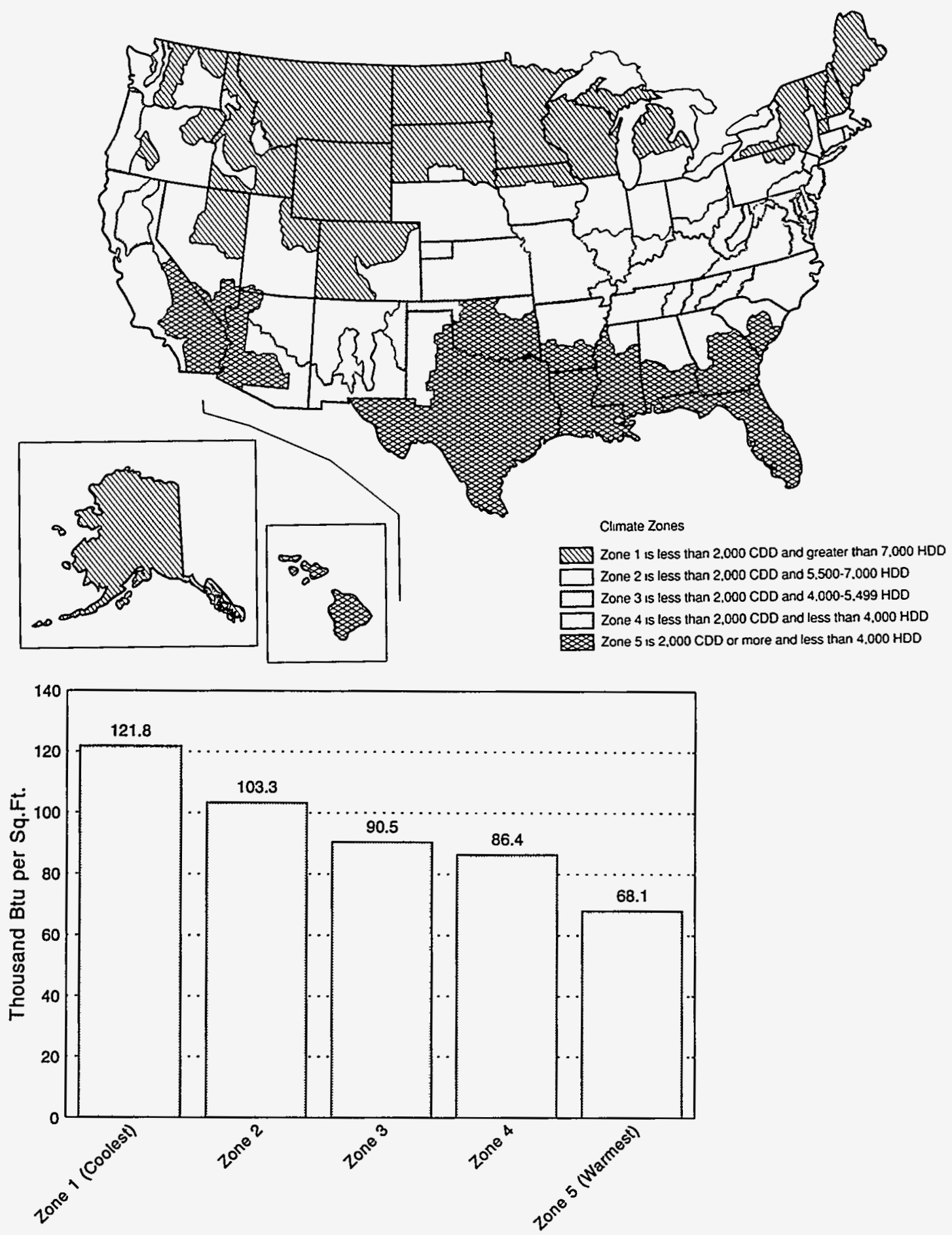

Source: Energy Information Administration, Office of Energy Markets and End Use, Forms ElA-871A through F of the 1989 Commercial Buildings Energy Consumption Survey.

- Figure 8 shows that the United States can be divided into climate zones according to the average number of heating degree-days (HDD's) and cooling degree-days (CDD's) that have occurred each year over a 45-year period. One HDD occurs when the temperature for the day averages one degree below 65 degrees Fahrenheit. One CDD occurs when the temperature for the day averages one degree above 65 degrees Fahrenheit.

- Zone 1 (the coolest) had the highest end-use intensity because of its greater demand for space heating. Conversely, Zone 5 (the warmest) had the lowest end-use intensity because of its small demand for space heating, even after taking into account the greater cooling load. 
Figure 9. End-Use Intensity Profiles, by Principal Building Activity, 1989

\section{a. Assembly}

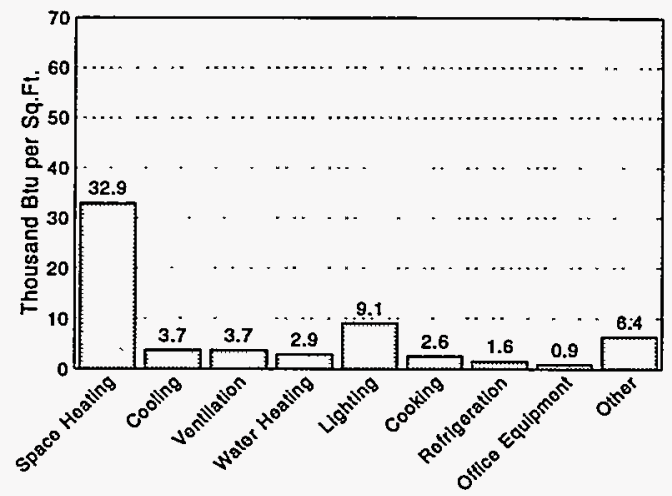

c. Food Sales

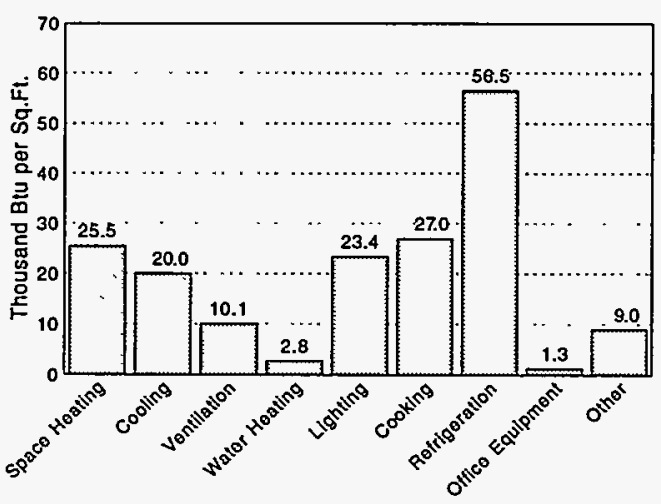

e. Health Care

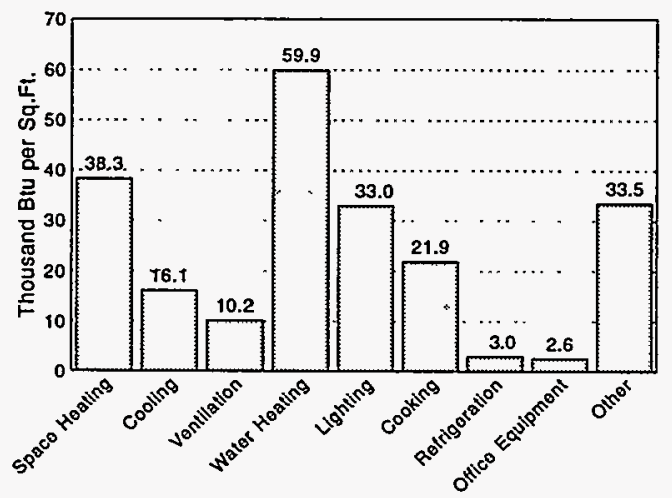

b. Education

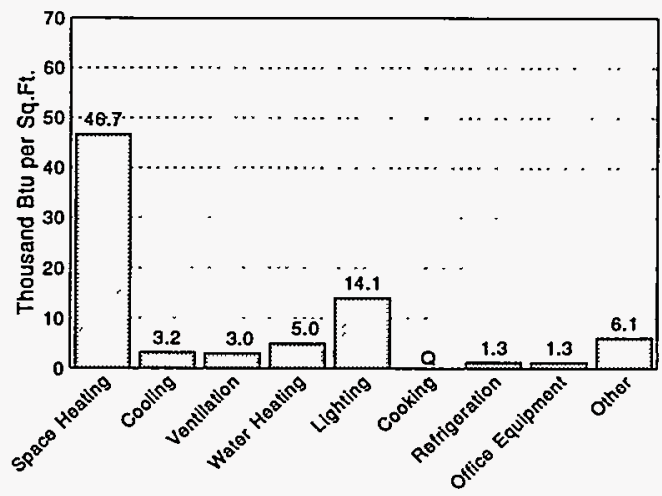

d. Food Service

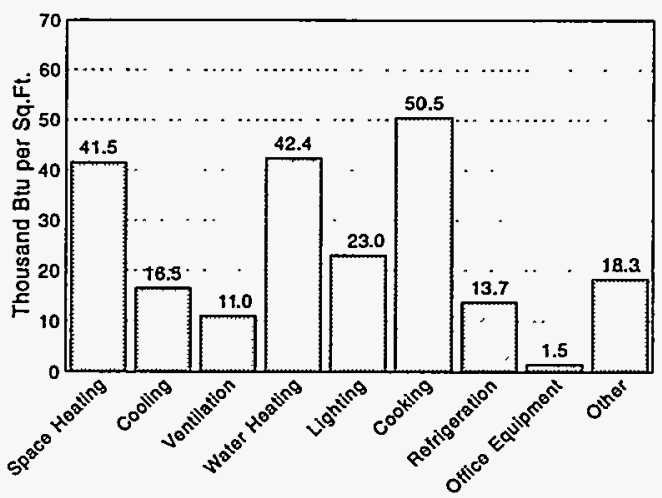

f. Lodging

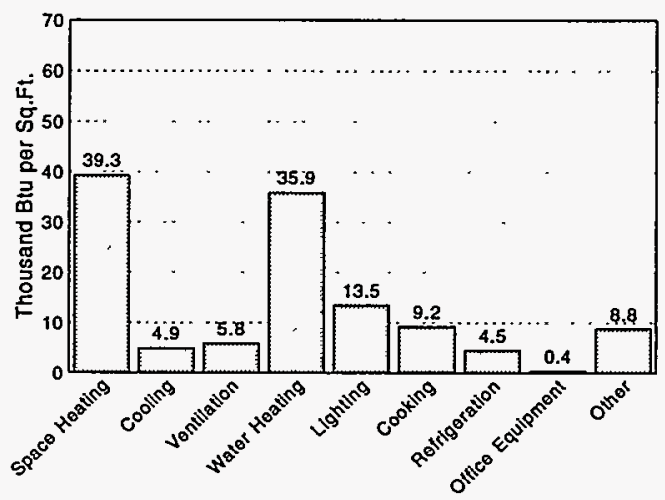




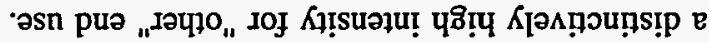

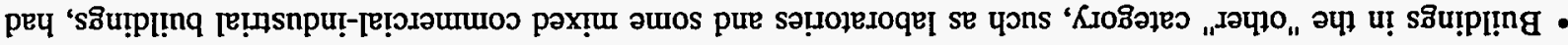

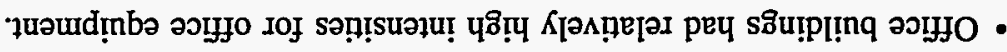

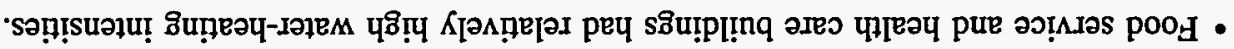

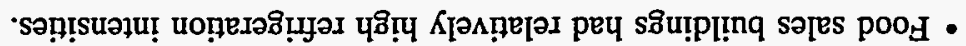

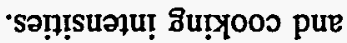

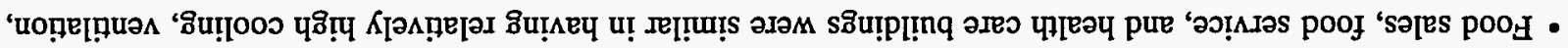

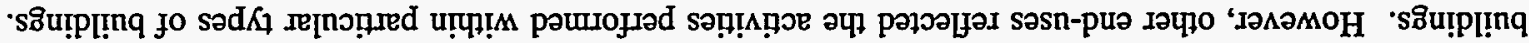

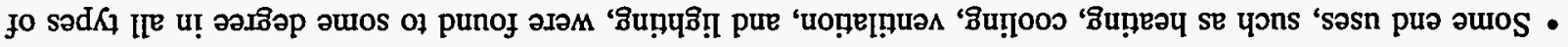

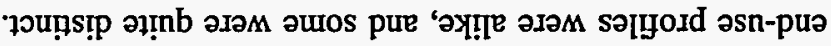

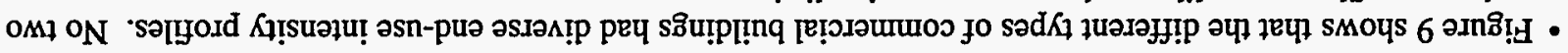

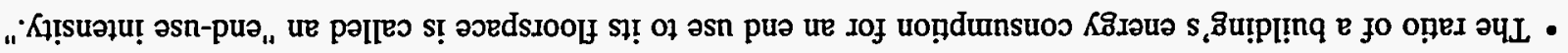

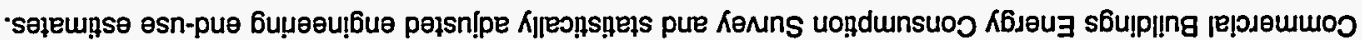

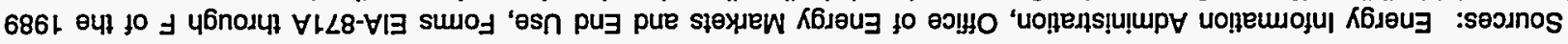

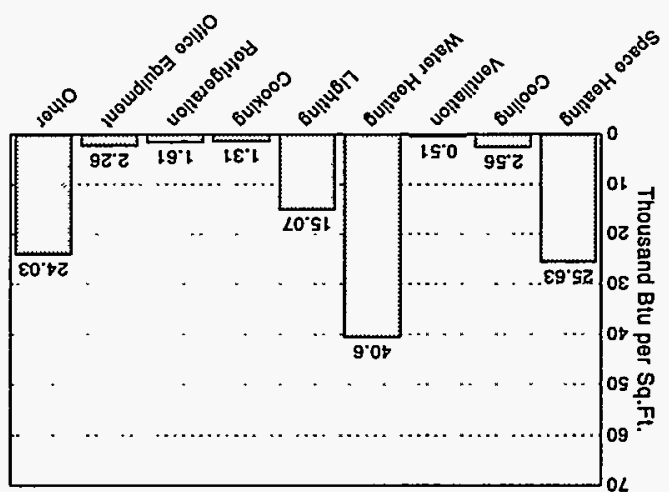

1อบा० !

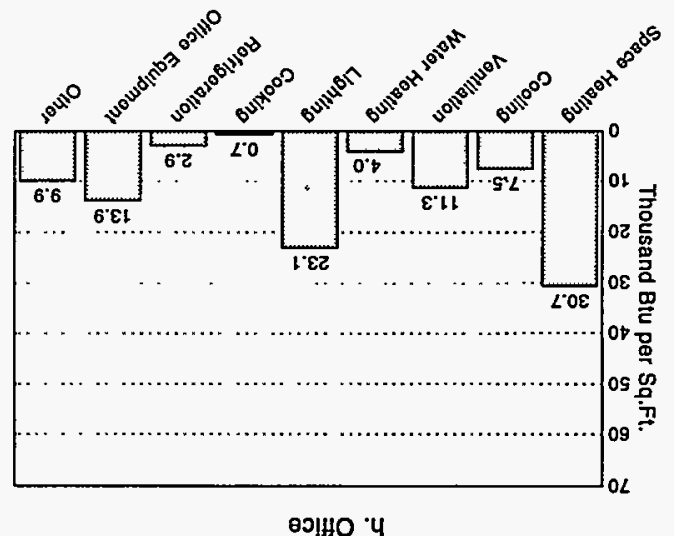

өอ!ก० '4

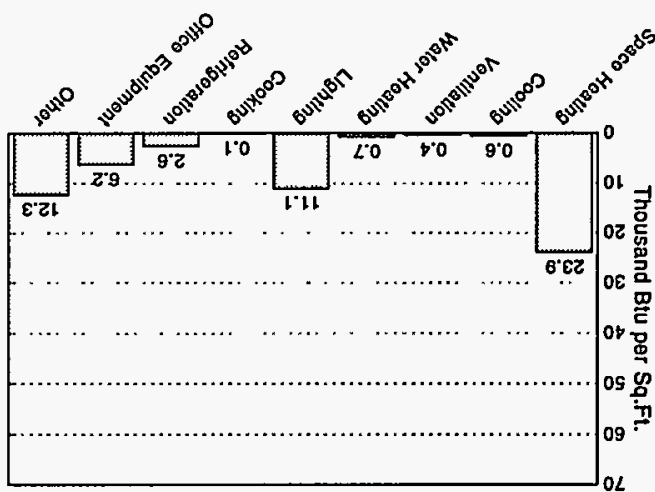

esnouəseM \%

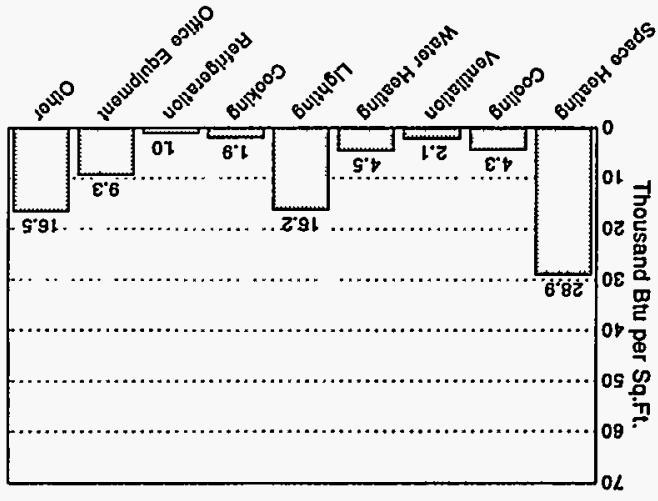

eopnes pue elluejow 6 


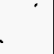




\section{Section 3. Space-Conditioning Intensities}

The purpose of this section is to provide information on how energy was used for space conditioning--heating, cooling, and ventilation-in commercial buildings. For heating, it shows the percent of energy supplied by each energy source and compares space-heating intensities for natural gas and electricity, by the year the building was constructed, and by principal building activity. It also gives the heating intensities in buildings where natural gas was the main heating fuel, by year constructed, floorspace, weekly operating hours, and climate zone. In addition, it shows heating intensities in buildings where electricity was the main heating source, by year constructed and floorspace. This section also shows electric cooling and ventilation intensities in commercial buildings, by year constructed, floorspace, principal building activity, and climate zone.

Key findings of this section include:

- Natural gas was the dominant energy source for space heating in commercial buildings in 1989, providing 63 percent of all the energy consumed for space heating (Figure 10).

- Natural gas provided a much higher percentage than electricity (whether measured by site or primary energy) of the space-heating energy consumed in buildings constructed during the 1980's, even though the amount of floorspace heated by the two energy sources was roughly equal (Figure 11).

- Natural gas space-heating intensities were higher than electricity intensities (site or primary). Possible explanations for the lower electricity intensities include the relatively higher prices of electricity (which may encourage more conservation measures, such as insulation) and the relatively younger age of electric spaceheating equipment (Figure 12).

- Education and food service buildings had relatively high natural gas space-heating intensities, either per square foot or per heated square foot-hour-HDD. Health care buildings had the lowest intensities per square foot-hourHDD (Figure 13).

- Buildings constructed in the 1970 's had the highest cooling intensities, defined as the ratio of energy used for cooling to the product of the cooled square footage, the annual building operating hours, and the average daily CDD's (Figure 17).

- Larger buildings had the highest ventilation intensities, defined as the ratio of energy used for ventilation to the product of the square footage and the annual building hours. More energy is required to circulate air through the relatively large interior spaces of the larger buildings (Figure 18). 


\section{Space Heating}

Figure 10. Percent of Energy Supplied for Space Heating, by Energy Source, 1989

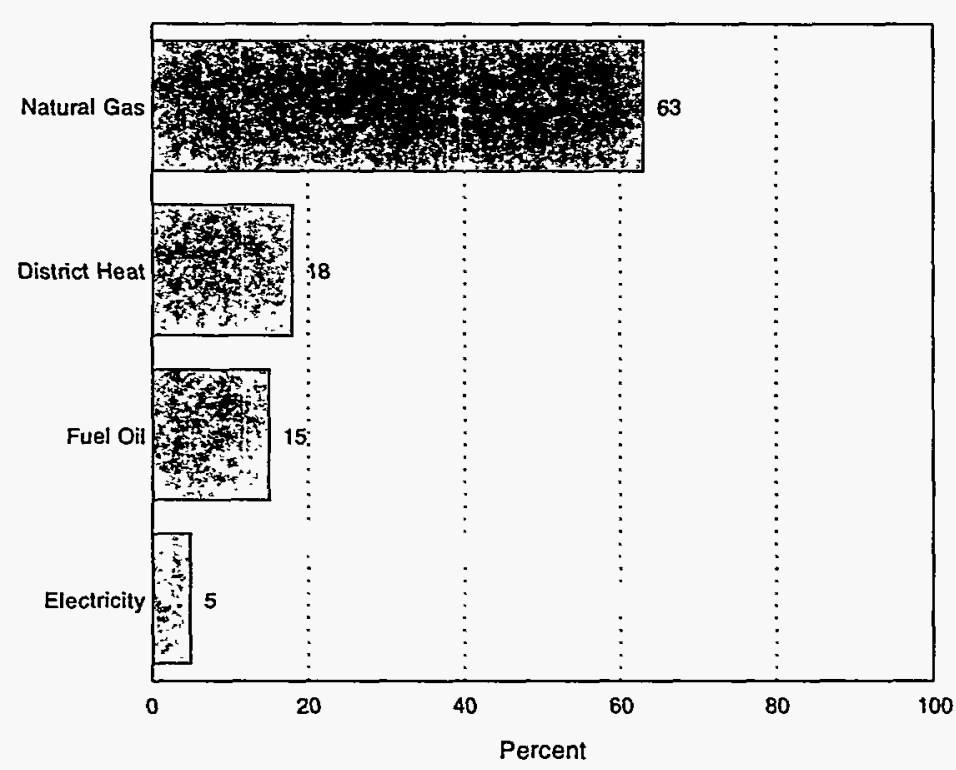

Note: Because of rounding, data may not sum to total.

Sources: Energy Information Administration, Office of Energy Markets and End Use, Forms ElA-871A through F of the 1989 Commercial Buildings Energy Consumption Survey and statistically adjusted engineering end-use estimates.

- Figure 10 shows that natural gas was the dominant energy source for space heating in commercial buildings in 1989, providing 63 percent of all the energy consumed for space heating.

- District heat and fuel oil lagged far behind at 18 percent and 15 percent, respectively, with electricity trailing at 5 percent.

- Buildings using natural gas as the main space-heating fuel consumed 1.2 quadrillion Btu for space heating, 21 percent of all energy delivered to commercial buildings in 1989.

- Electricity, like fuel oil, was widely used both for main space heating and for secondary (supplemental or backup) space heating. In 1989, 75 trillion Btu of electricity were used for main space heating, while the rest (21 trillion Btu) was used for secondary space heating. 
Figure 11. Percent of Heated Floorspace Served by Each Main Space-Heating Energy Source and Percent of SpaceHeating Consumption, by Year Constructed, 1989
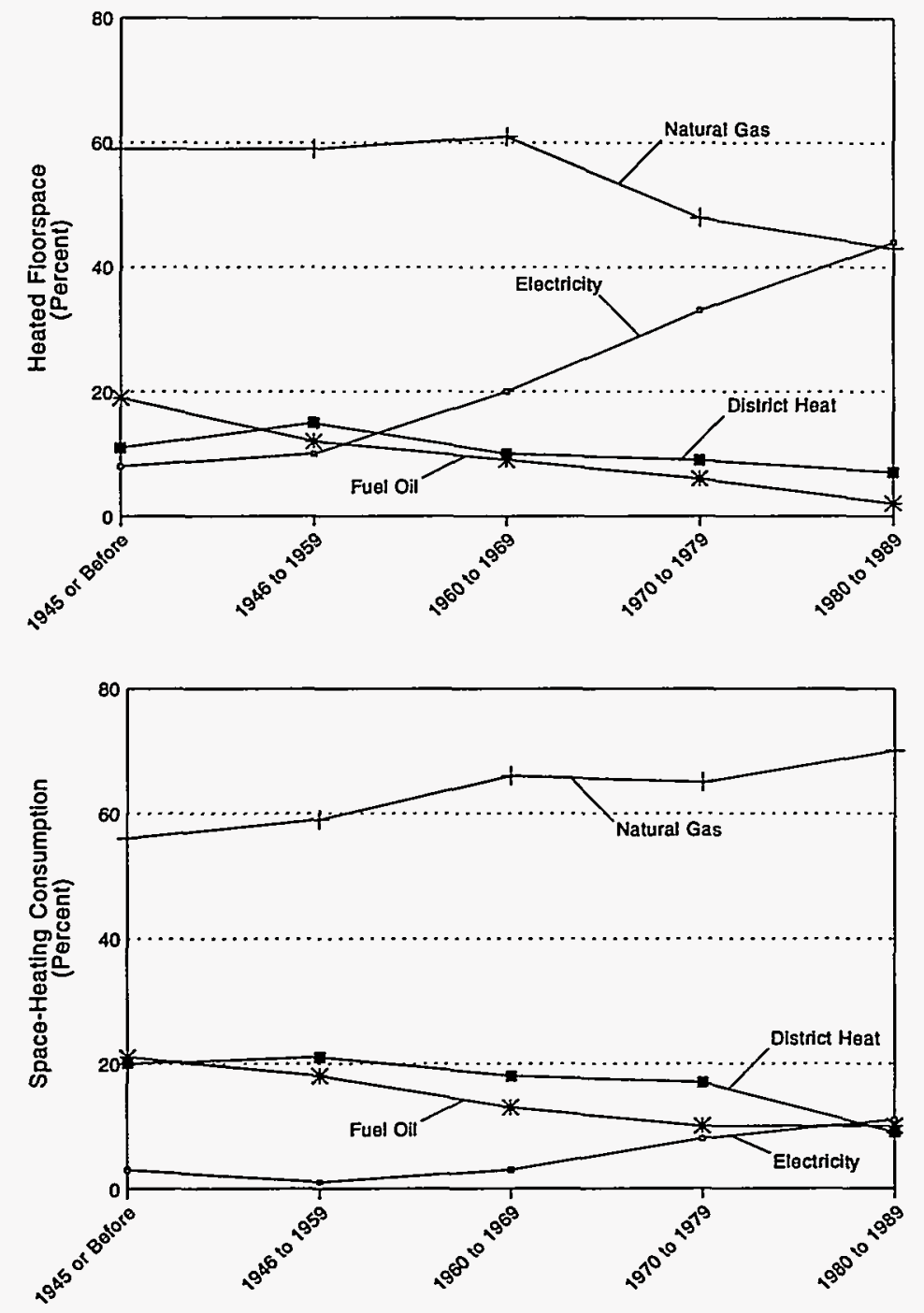

Sources: Energy Information Administration, Office of Energy Markets and End Use, Forms ElA-871A through F of the 1989 Commercial Buildings Energy Consumption Survey and statistically adjusted engineering end-use estimates.

- Figure 11 shows that natural gas provided a much higher percentage than electricity of the space-heating energy used in buildings constructed during the 1980's, even though the amount of floorspace heated by the two energy sources was roughly equal.

- The bulk of new construction during the 1980's occurred in warmer climates. In warmer climates, where space-heating demands were relatively lower, electricity tended to be the main heating energy source. Natural gas continued to be used in climates with higher space-heating demands. 
a. Intensity per Square Foot

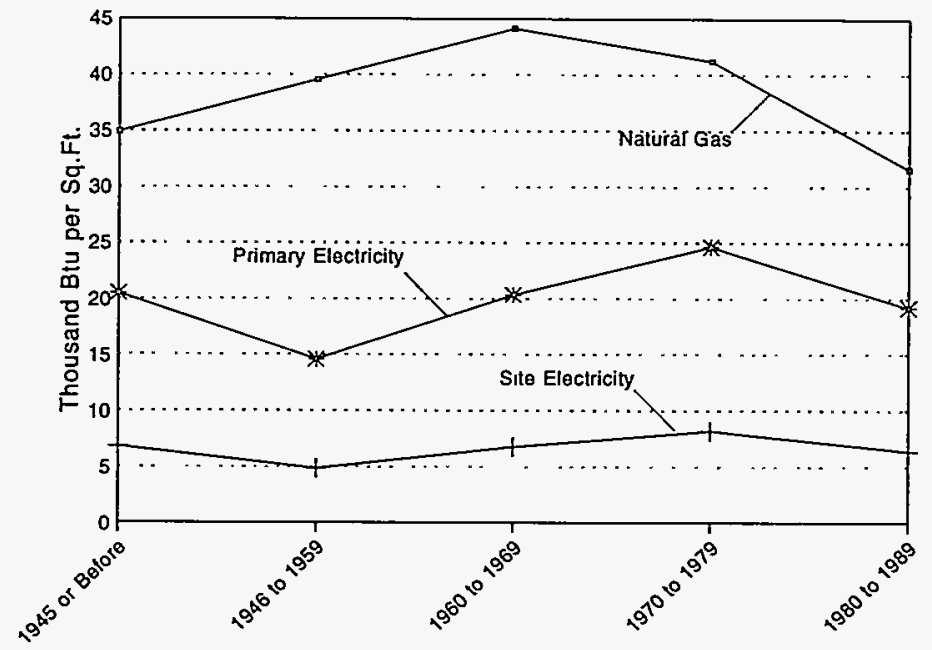

b. Intensity per Square Foot-Operating Hour-HDD

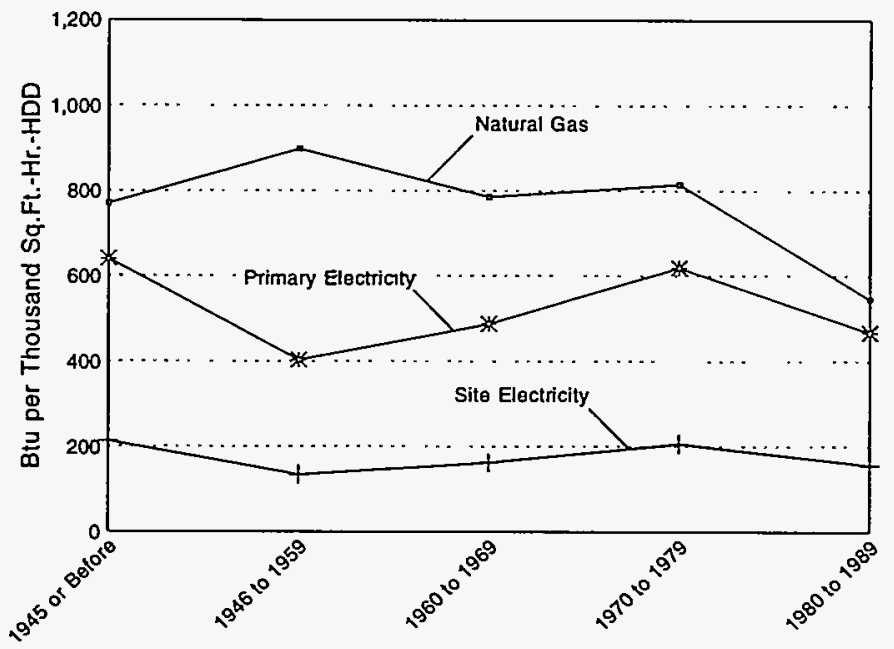

Sources: Energy Information Administration, Office of Energy Markets and End Use, Forms ElA-871A through F of the 1989 Commercial Buildings Energy Consumption Survey and statistically adjusted engineering end-use estimates.

- Figure 12 shows that no matter which measurement is used--energy consumption per square foot or energy consumption per square foot-operating hour-HDD--natural gas space-heating intensities were higher than site electricity intensities.

- Even after converting site electricity to primary energy, the electricity space-heating intensities would still be lower than those for natural gas. However, the gap between primary electricity and natural gas space-heating intensities was narrower in newer buildings, especially when differences in weather (HDD's) and operating hours are taken into account.

- Possible explanations for the lower electricity intensities include the relatively higher prices of electricity (which may encourage more conservation measures, such as insulation) and the relatively younger age of electric spaceheating equipment. 
Figure 13. Comparison of Main Space-Heating Intensities for Natural Gas and Electricity, by Principal Building Activity, 1989

a. Intensity per Square Foot

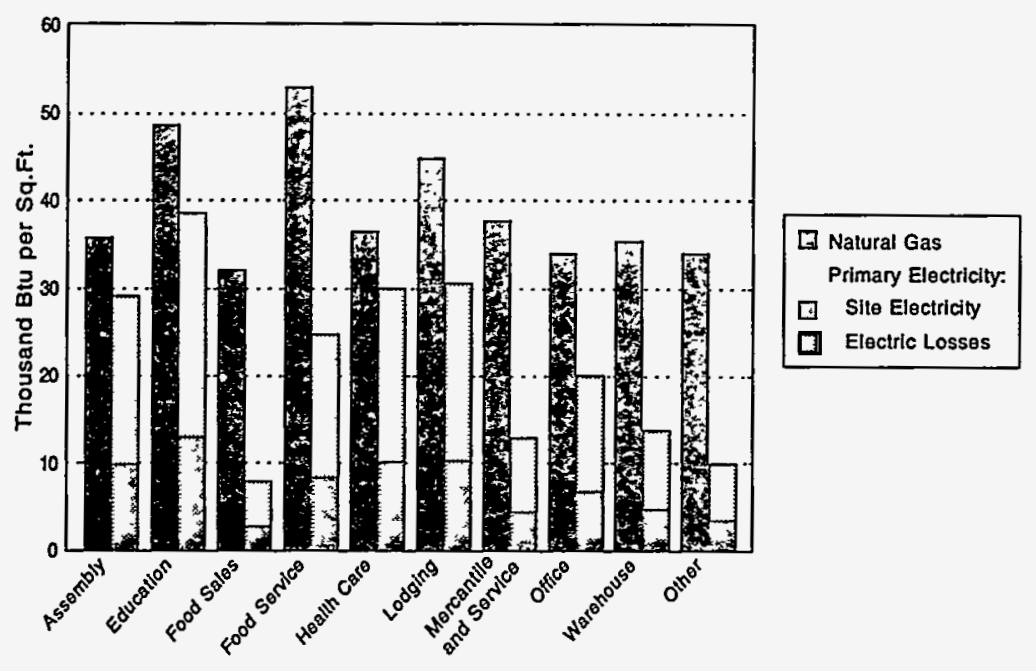

b. Intensity per Square Foot-Operating Hour-HDD

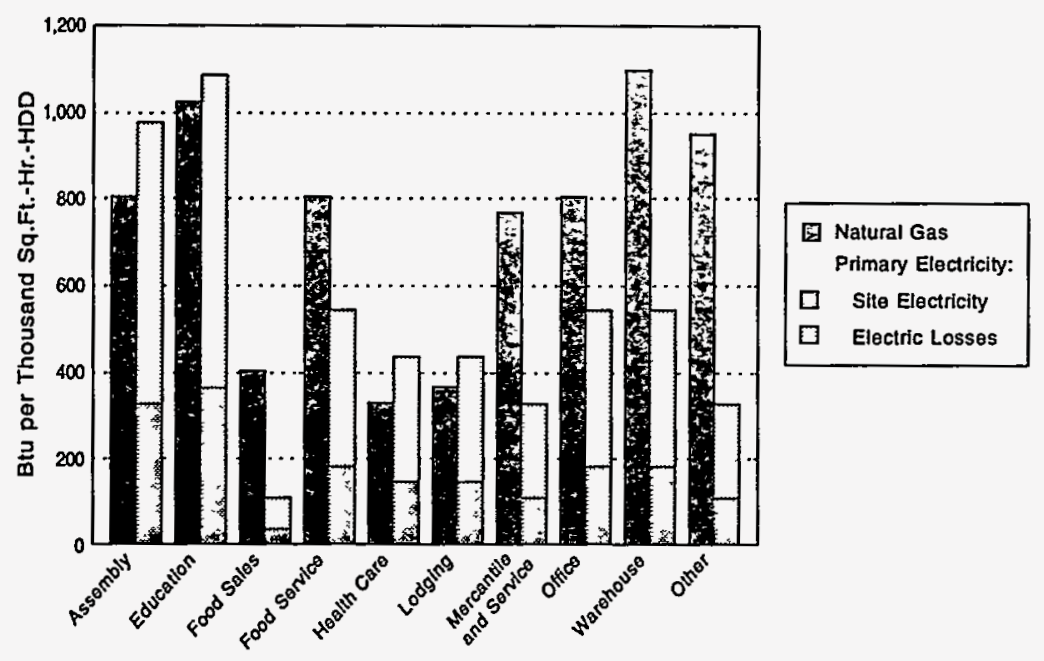

Sources: Energy Information Administration, Office of Energy Markets and End Use, Forms EIA-871A through F of the 1989 Commercial Buildings Energy Consumption Survey and statistically adjusted engineering end-use estimates.

- Figure 13 shows that natural gas space-heating intensities were higher than site electricity space-heating intensities in all types of buildings. However, electricity intensities were somewhat higher than natural gas intensities for assembly, education, health care, and lodging buildings when electric losses were included.

- Education and food service buildings had relatively high natural gas space-heating intensities, either per square foot or per heated square foot-hour-HDD.

- Natural gas intensities per square foot were about the same in health care buildings as in warehouses. However, health care buildings had the lowest intensities per heated square foot-hour-HDD, while warehouses had the highest. Warehouses consume energy more intensively to meet a much smaller demand (particularly in terms of operating hours and proportion of floorspace heated) than do health care buildings. 
Figure 14. Heating Intensities in Buildings Where Natural Gas Was Main Heating Fuel, by Year Constructed and by Floorspace, 1989

a. Year Constructed

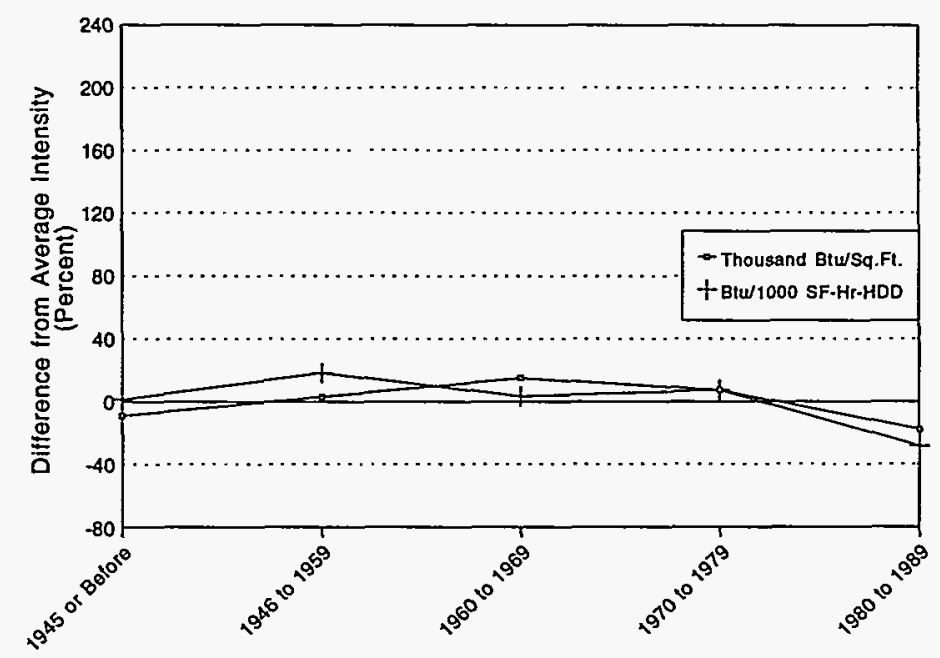

b. Floorspace

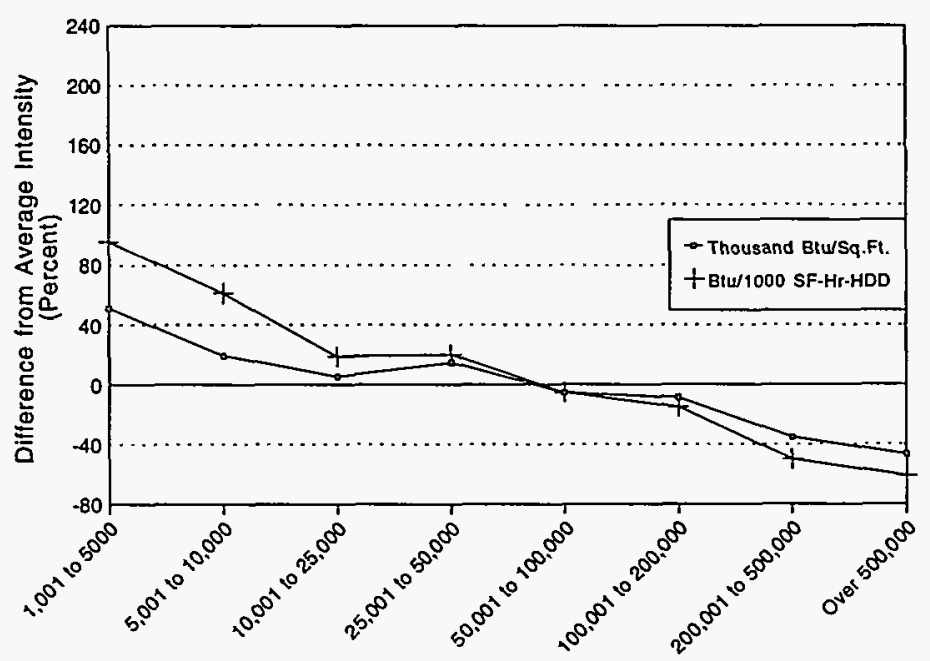

Sources: Energy Information Administration, Office of Energy Markets and End Use, Forms ElA-871A through F of the 1989 Commercial Buildings Energy Consumption Survey and statistically adjusted engineering end-use estimates.

- Figure 14 shows that buildings constructed in the 1960 's, when energy was plentiful and cheap, continued to have high natural gas heating intensities in 1989.

- In buildings constructed after the energy crisis of the early $1970^{\prime}$ s, heating intensities fell steadily until the mid1980's.

- The larger a building's floorspace, the lower was its heating intensity. This phenomenon is probably due to the fact that since the ratio of exterior surface to total floorspace was smaller in larger buildings, building interiors required less heating to maintain a comfortable temperature. 
Figure 15. Heating Intensities in Buildings Where Natural Gas Was Main Heating Fuel, by Weekly Operating Hours and by Climate Zone, 1989

a. Weekly Operating Hours

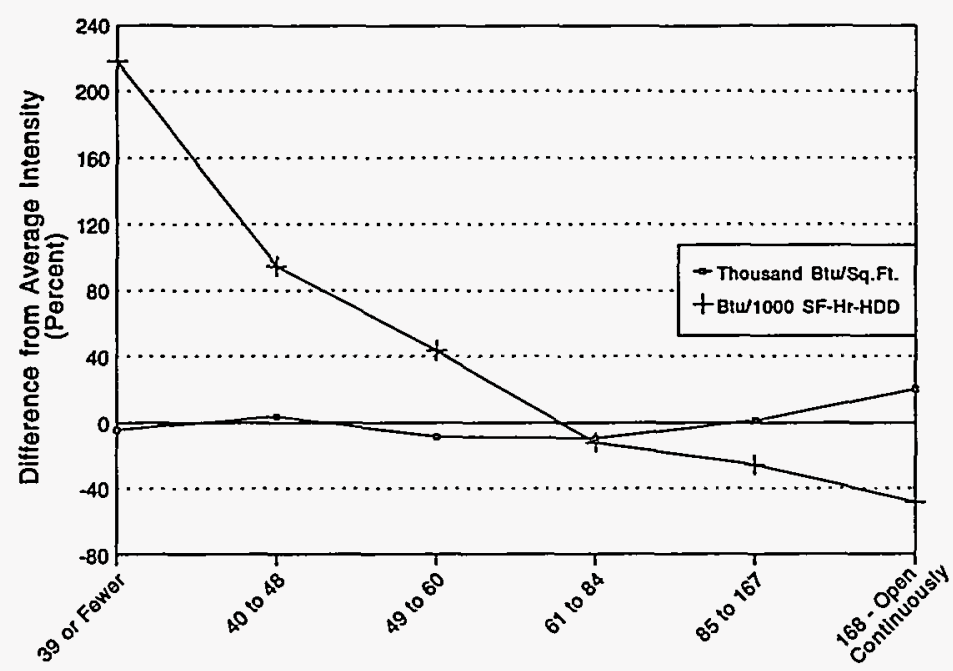

b. Climate Zone

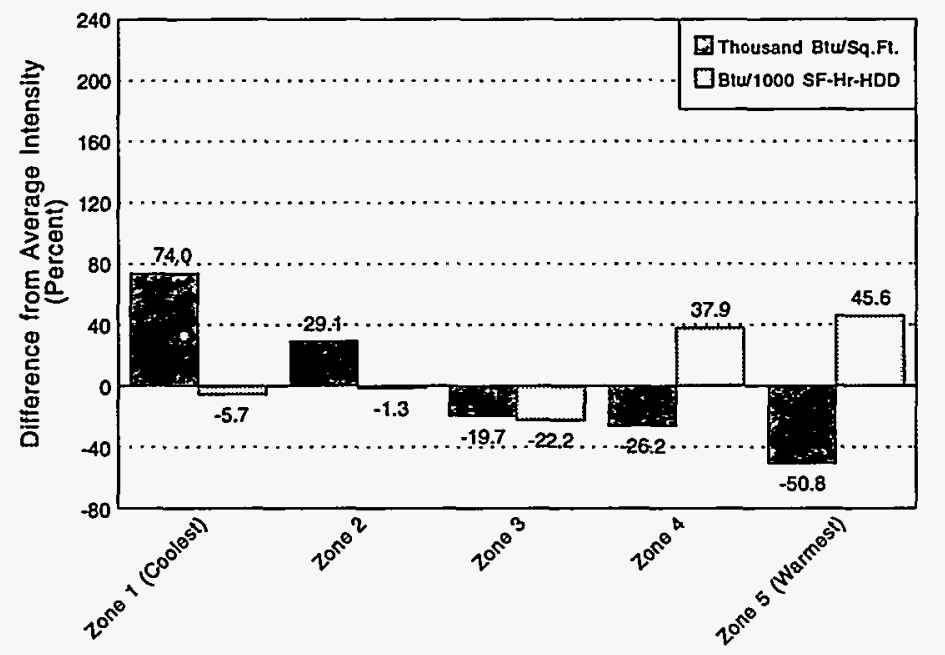

Sources: Energy Information Administration, Office of Energy Markets and End Use, Forms ElA-871A through F of the 1989 Commercial Buildings Energy Consumption Survey and statistically adjusted engineering end-use estimates.

- Figure 15 shows that the fewer the number of operating hours, the higher the space-heating intensity (square foot-hour-HDD). This could indicate that heating systems in buildings with shorter weekly schedules tended to operate during off-hours.

- Heating intensity per square foot declined from the coolest to the warmest climate zone. However, intensity per square foot-HDD was relatively constant in the coolest three zones and increased in the warmest two zones, where heating demand was lowest. Buildings in warmer areas consumed relatively more energy in cooler weather than did buildings in colder climates, due either to differences in equipment efficiencies or to differences in the occupants' demands for space heating. 
Figure 16. Heating Intensities in Buildings Where Electricity Was Main Heating Fuel, by Year Constructed and by Floorspace, 1989

a. Year Constructed

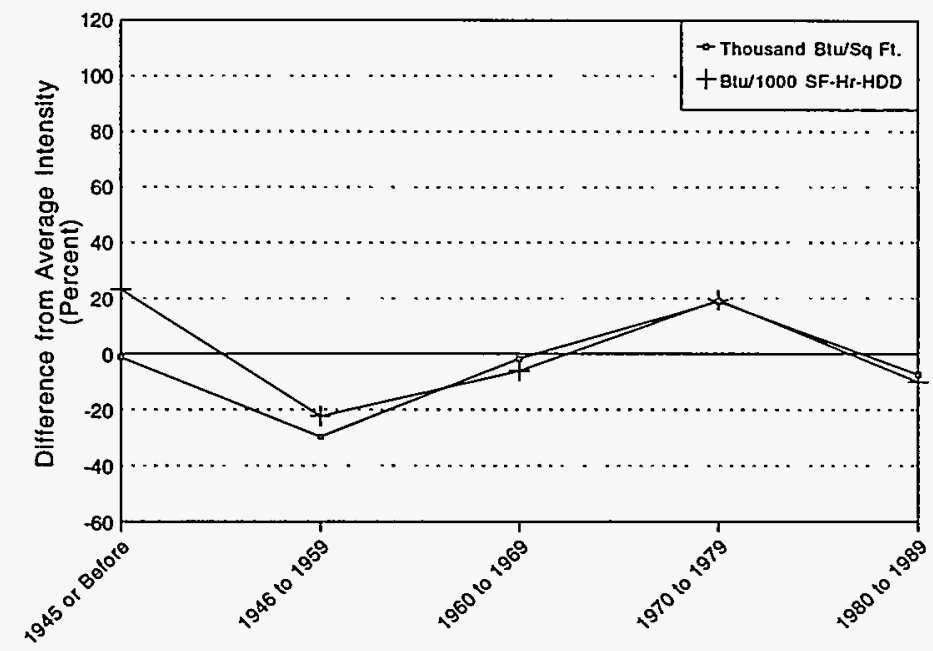

b. Floorspace

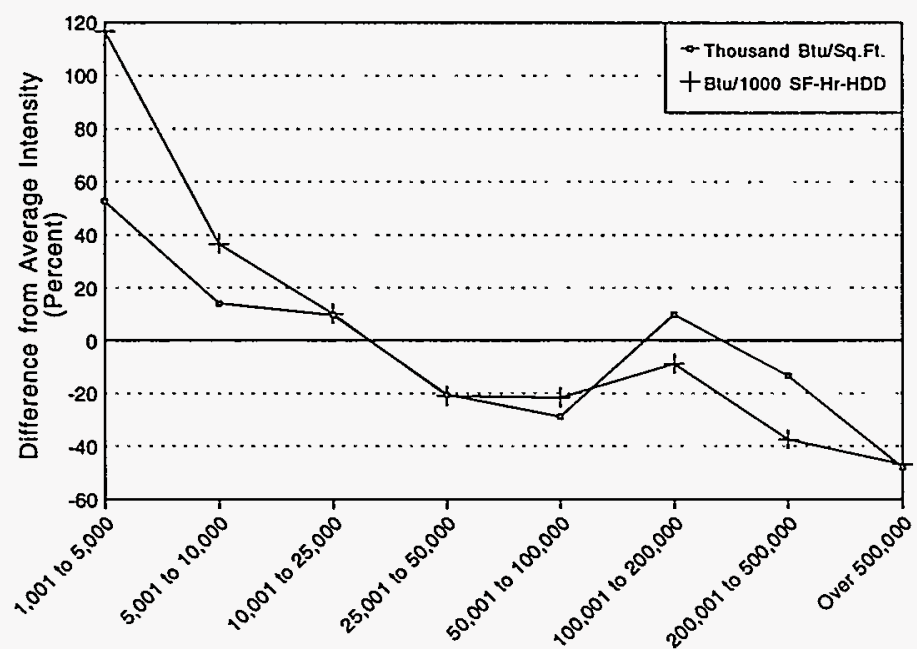

Sources: Energy Information Administration, Office of Energy Markets and End Use, Forms ElA-871A through F of the 1989 Commercial Buildings Energy Consumption Survey and statistically adjusted engineering end-use estimates.

- Figure 16 shows that electricity heating intensities were highest for buildings constructed during the 1970's.

- Electricity heating intensities declined sharply for building constructed during the 1980's.

- As was the case for natural gas, electricity heating intensities declined as building size increased. 


\section{Cooling and Ventilation}

Figure 17. Electric Cooling and Ventilation Intensities, by Year Constructed, 1989

a. Cooling

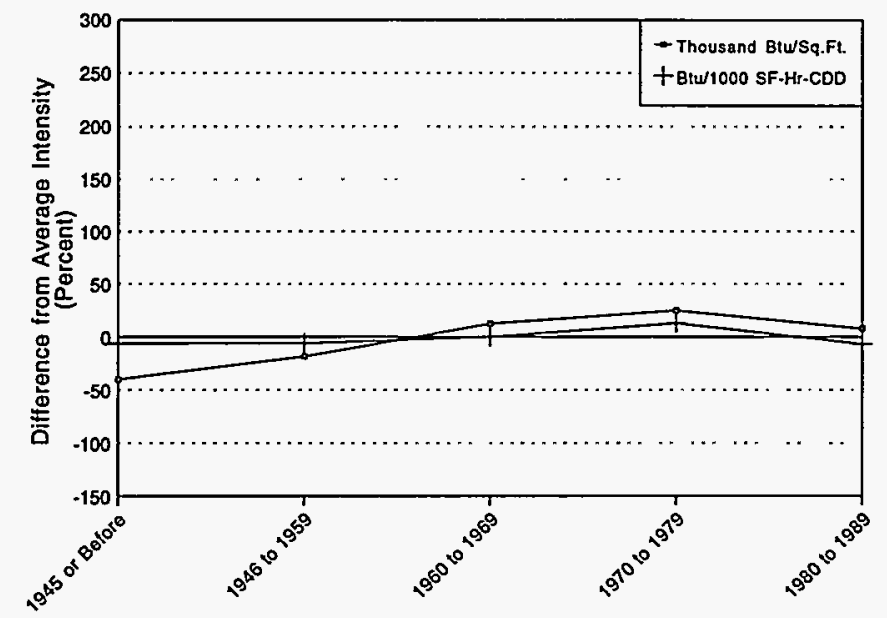

b. Ventilation

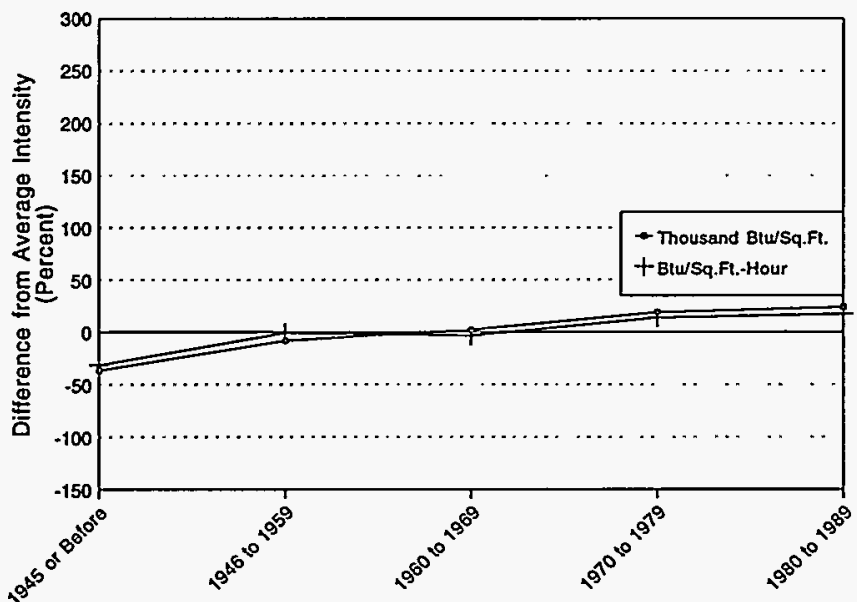

Sources: Energy Information Administration, Office of Energy Markets and End Use, Forms EIA-871A through F of the 1989 Commercial Buildings Energy Consumption Survey and statistically adjusted engineering end-use estimates.

- Cooling intensities are defined as the ratio of energy used for cooling to the product of the cooled square footage, the annual building operating hours, and the average daily CDD's.

- Figure 17 shows that the oldest buildings had the lowest cooling intensities.

- Cooling intensities increased to a peak for buildings constructed in the 1970 's.

- Ventilation intensities are defined as the ratio of energy used for ventilation to the product of the square footage and the annual building operating hours.

- The oldest buildings had the lowest ventilation intensities.

- Buildings constructed during the 1980 's had the highest ventilation intensities. 
Figure 18. Electric Cooling and Ventilation Intensities, by Floorspace, 1989

a. Cooling

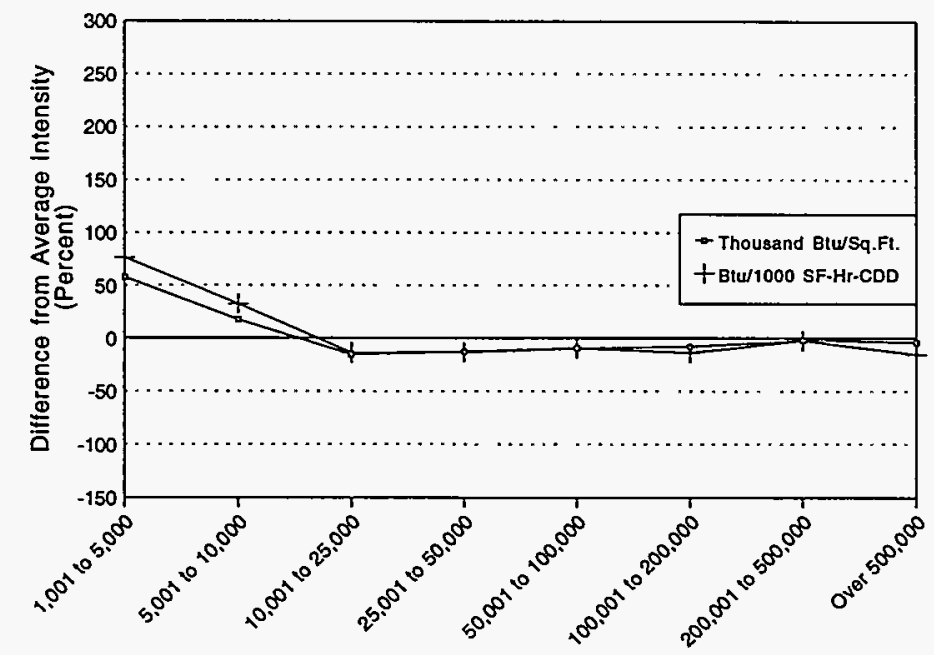

b. Ventilation

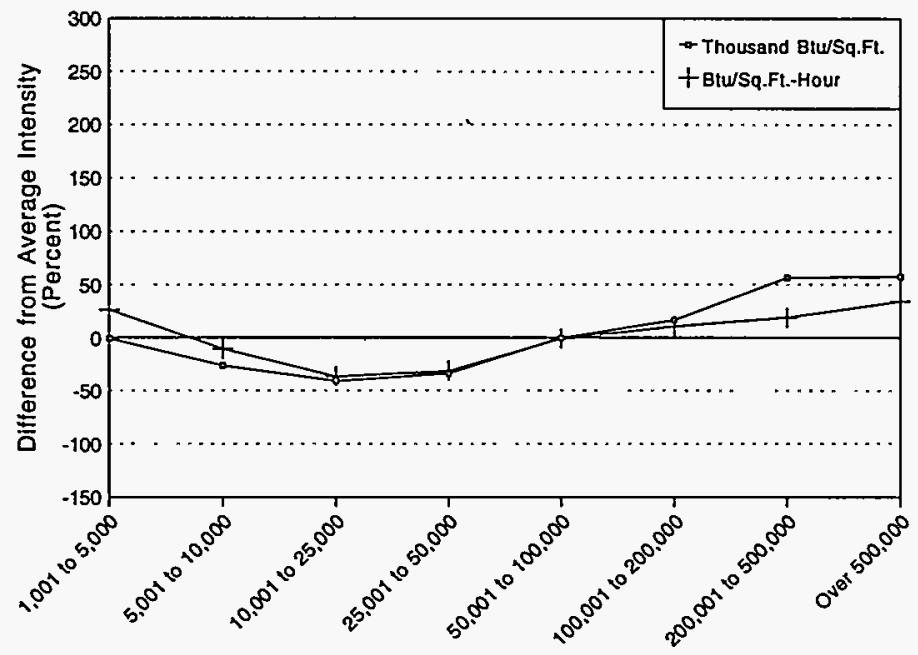

Sources: Energy Information Administration, Office of Energy Markets and End Use, Forms ElA-871A through F of the 1989 Commercial Buildings Energy Consumption Survey and statistically adjusted engineering end-use estimates.

- Figure 18 shows that cooling intensities, like space-heating intensities, were higher for the smallest buildings. As with space heating, the larger buildings had proportionately less exposed exterior surface area, relative to their total floorspace.

- Ventilation intensities were high for the smallest buildings (as was true for heating and cooling intensities), but were the highest for the larger buildings. More ventilation was required in larger buildings, particularly in buildings such as offices and hospitals. 
a. Cooling

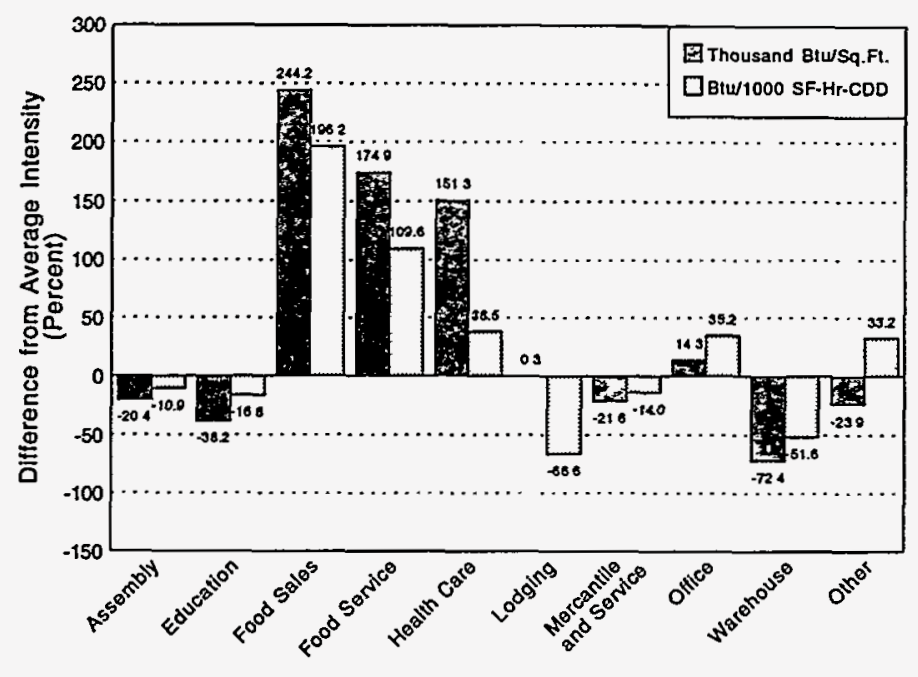

b. Ventilation

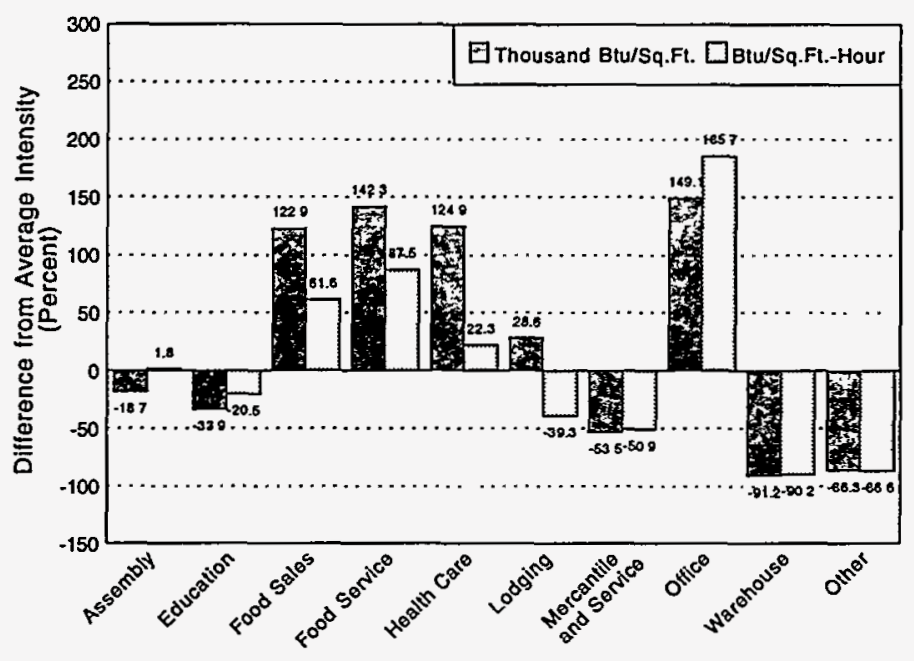

Sources: Energy Information Administration, Office of Energy Markets and End Use, Forms ElA-871A through F of the 1989 Commercial Buildings Energy Consumption Survey and statistically adjusted engineering end-use estimates.

- Figure 19 shows that cooling intensities in food sales, food service, and health care buildings were relatively high.

- High ventilation intensities were associated with high cooling intensities in food sales, food service, and health care buildings.

- The highest ventilation intensities were found among office buildings, which included a substantial number of larger buildings and had the highest occupant densities (workers per thousand square feet). 
Figure 20. Electric Cooling and Ventilation Intensities, by Climate Zone, 1989

a. Cooling

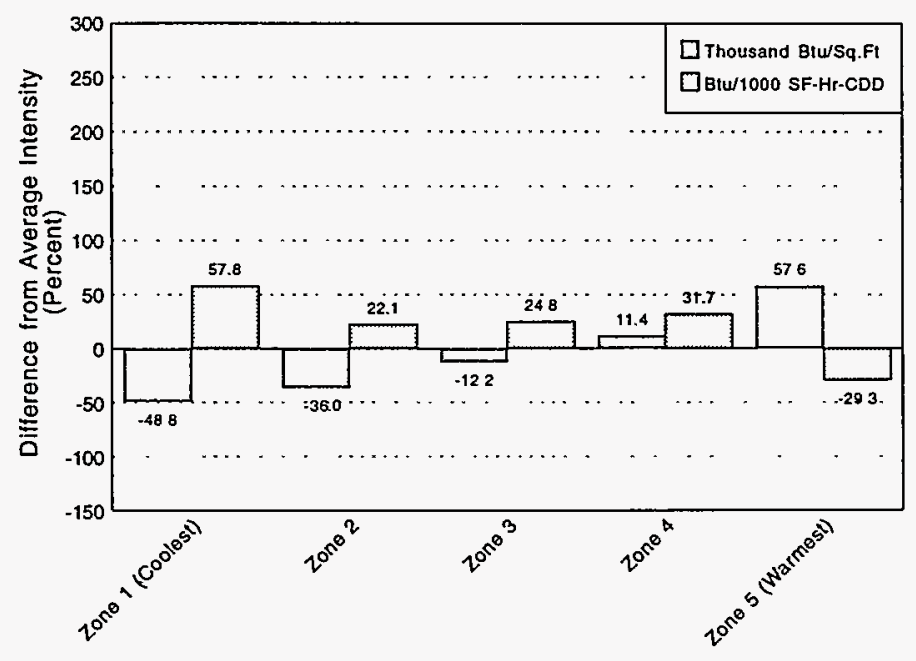

b. Ventilation

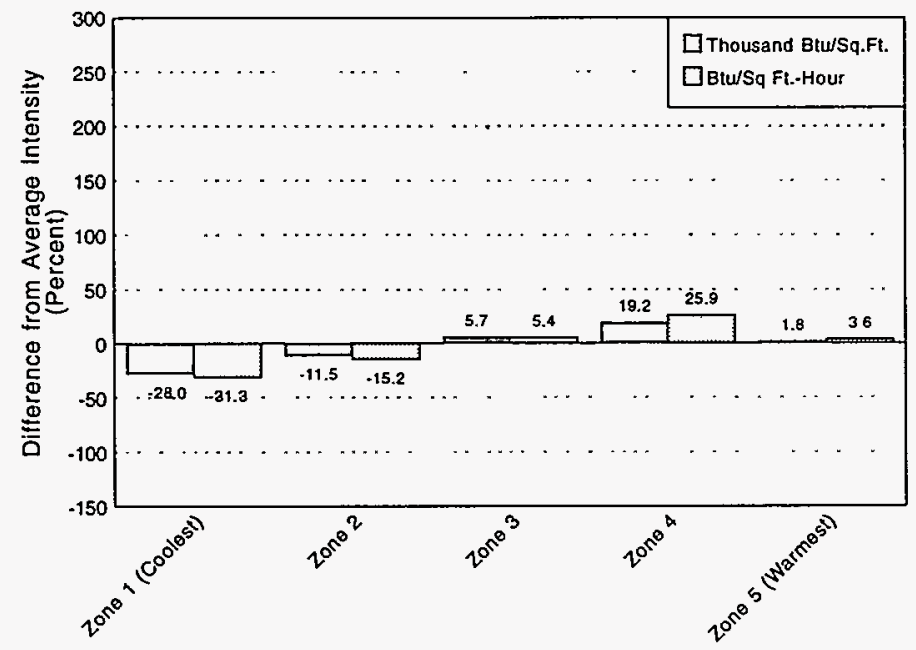

Sources: Energy Information Administration, Office of Energy Markets and End Use, Forms ElA-871A through F of the 1989 Commercial Buildings Energy Consumption Survey and statistically adjusted engineering end-use estimates.

- Figure 20 shows that cooling intensities per square foot increased from the coolest to the warmest climate. However, intensities per square foot-hour-CDD were the lowest in the warmest climate zone, where the cooling demand was greatest. Buildings in cooler areas consumed relatively more energy for cooling in warmer weather than did buildings in warmer climates, due either to differences in equipment efficiencies or to differences in the occupants' demands for cooling.

- Ventilation intensities were lowest in the coolest climate zone. However, climate zone was not as important for ventilation intensity as it was for cooling intensity. 


\section{Section 4. Intensities for Other End Uses}

The purpose of this section is to provide information on intensities for end uses other than space conditioning, including lighting, water heating, cooking, refrigeration, office equipment, and miscellaneous uses. These other end uses accounted for 55 percent of all energy consumed in commercial buildings in 1989. This section shows lighting intensities by year of construction, floorspace, and principal building activity. It also shows intensities for natural gas water heating and cooking and for electric refrigeration, by principal building activity. In addition, it shows electric intensities for office equipment.

Key findings of this section include:

- The oldest buildings had the lowest lighting intensity, defined either as (1) the ratio of the amount of energy consumed for lighting to the square footage of building floorspace or (2) the ratio of the amount of energy consumed for lighting to the square footage of lighted floorspace (Figure 21).

- Buildings constructed during the 1970's and 1980's had the highest lighting intensities (Figure 21).

- Office buildings had the highest lighting intensities per lighted square foot-hour (Figure 23).

- Food service and health care buildings had the highest water-heating intensities per square foot--more than five times the average for all buildings (Figure 24).

- Food sales and food service buildings had the highest energy intensities for cooking and refrigeration (Figure 24).

- Office buildings had the highest intensities for office-equipment energy use (Figure 25).

- Buildings constructed during the 1980's had the highest intensities for office-equipment energy use (Figure 25). 


\section{Lighting}

Figure 21. Lighting Intensities, by Year Constructed, 1989

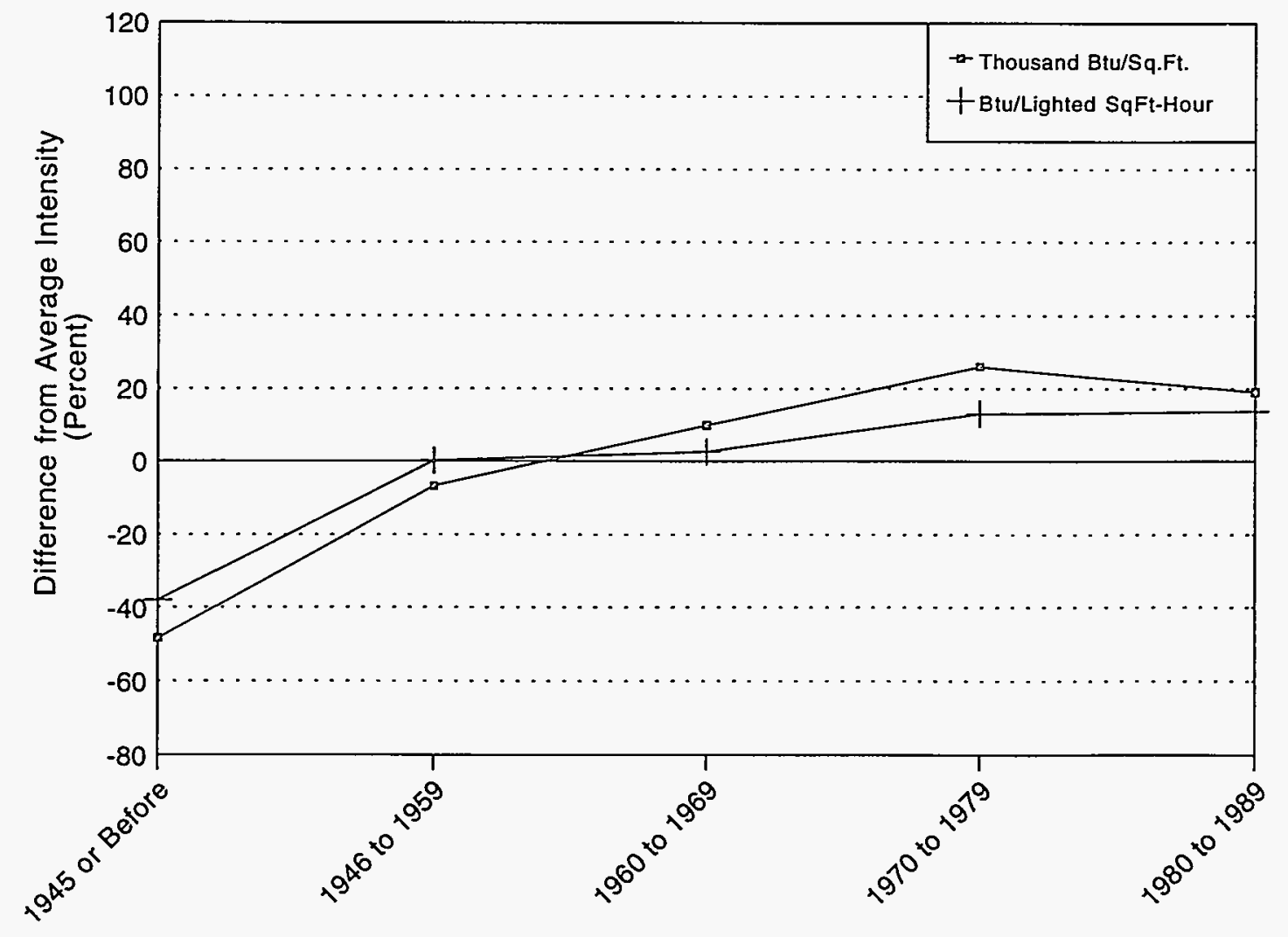

Sources: Energy Information Administration, Office of Energy Markets and End Use, Forms EIA-871A through F of the 1989 Commercial Buildings Energy Consumption Survey and statistically adjusted engineering end-use estimates.

- Lighting intensity can be defined either as (1) the ratio of the amount of energy consumed for lighting to the square footage of building floorspace or (2) the ratio of the amount of energy consumed for lighting to the product of the square footage of lighted floorspace and the operating hours.

- Figure 21 shows that the oldest buildings had the lowest lighting intensity.

- Buildings constructed during the 1970's and 1980's had the highest lighting intensities.

- Lighting intensity per lighted square foot-hour was lower than intensity per square foot among the more recently constructed buildings. This indicates that the rise in intensity per square foot can be partly explained by increasing operating hours. 
Figure 22. Lighting Intensities, by Floorspace, 1989

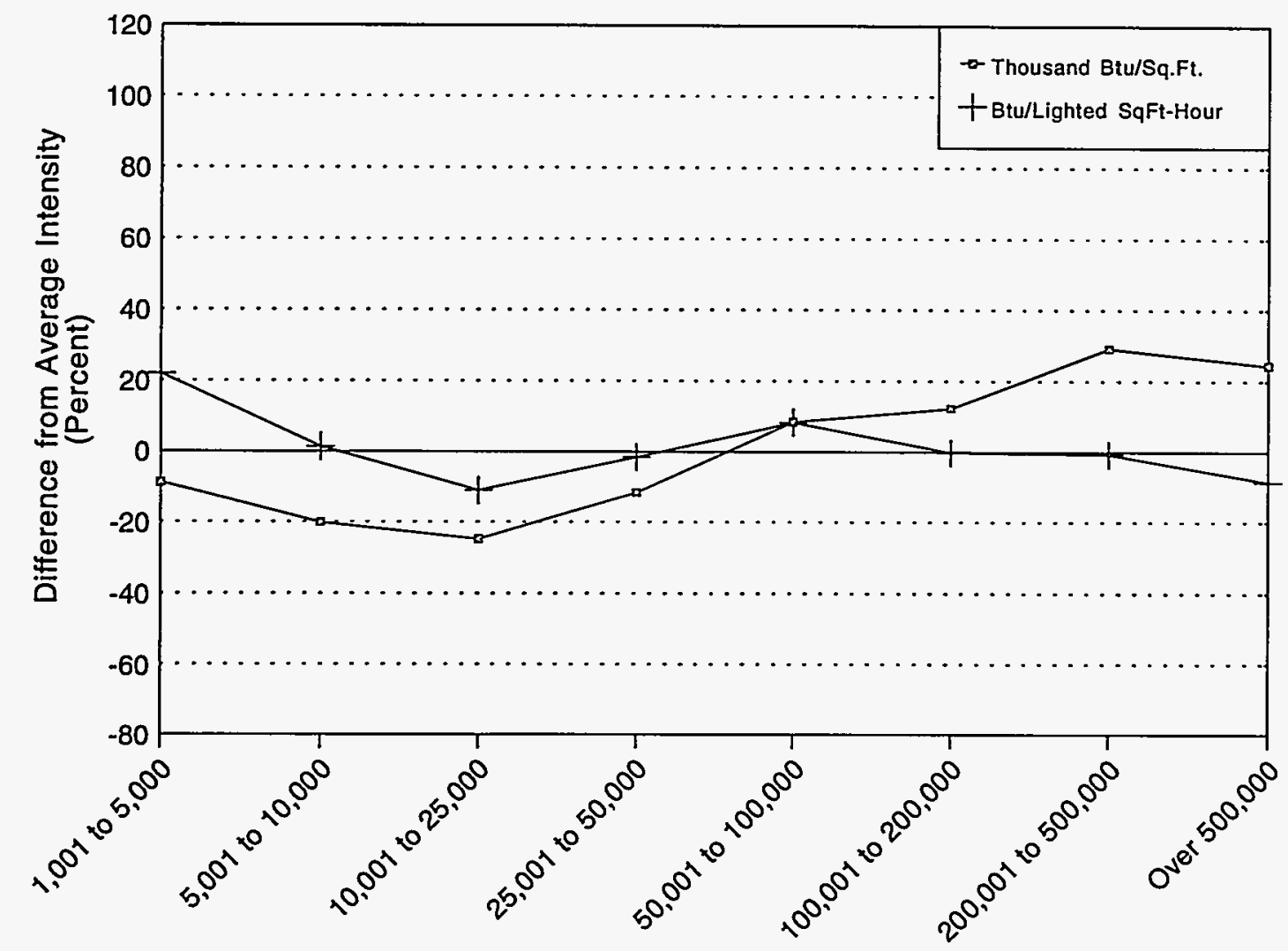

Sources: Energy Information Administration, Office of Energy Markets and End Use, Forms ElA-871A through F of the 1989 Commercial Buildings Energy Consumption Survey and statistically adjusted engineering end-use estimates.

- Figure 22 shows that lighting intensity per lighted square foot-hour remained relatively constant for all sizes of buildings.

- Lighting intensity per square foot was relatively low for the smaller buildings, and relatively high for larger buildings, reflecting the general tendency for smaller buildings to have shorter operating hours than larger buildings. 
Figure 23. Lighting Intensities, by Principal Building Activity, 1989

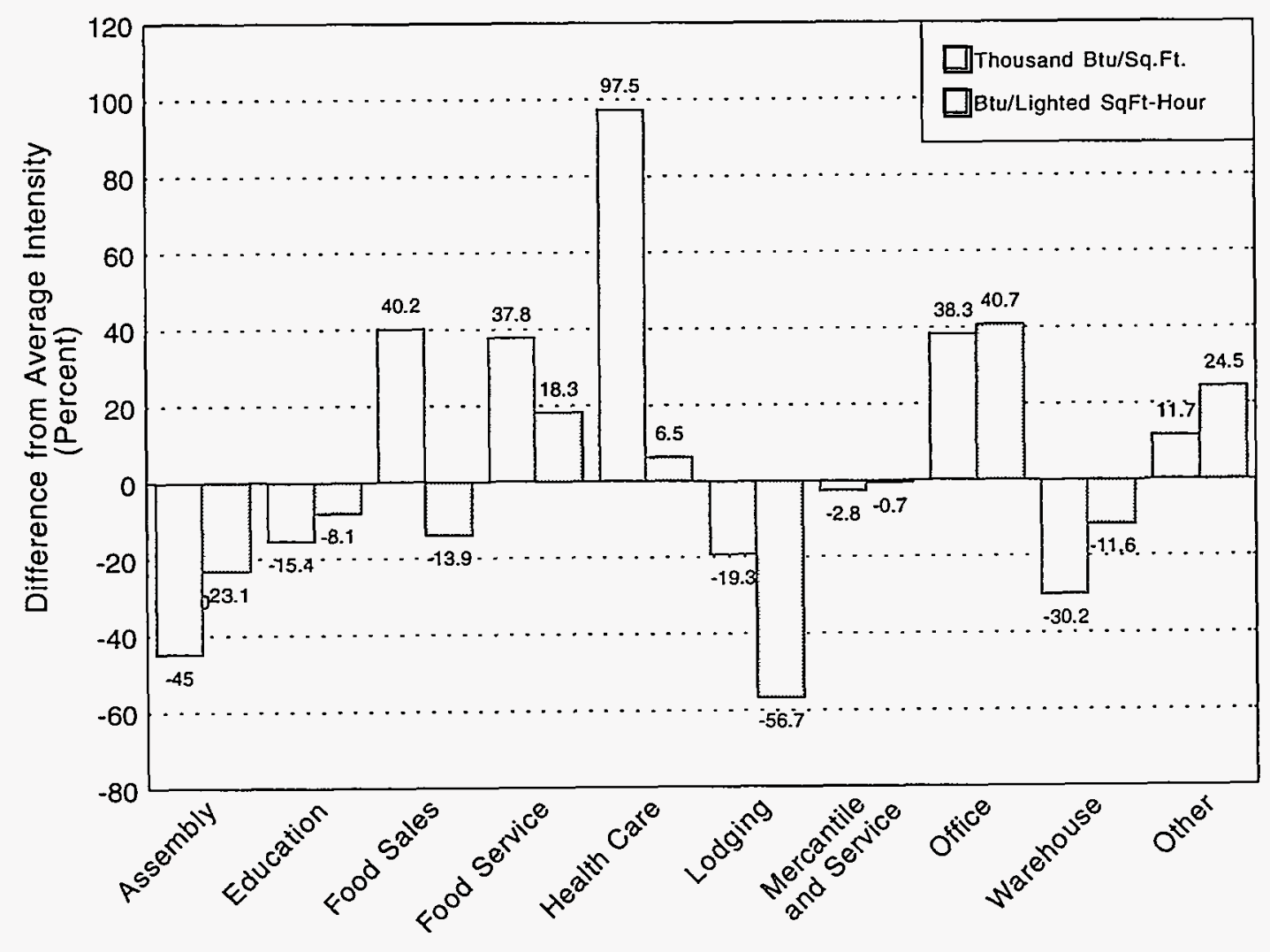

Sources: Energy Information Administration, Office of Energy Markets and End Use, Forms EIA-871A through F of the 1989 Commercial Buildings Energy Consumption Survey and statistically adjusted engineering end-use estimates.

- Figure 23 shows that office buildings had the highest lighting intensities per lighted square foot-hour. Food service and "other" buildings also had high intensities.

- Although health care buildings had the highest lighting intensity per square foot, their intensity per lighted square foot-hour was only slightly above average. Health care buildings' high intensity per square foot resulted from relatively long operating hours. 


\section{All Other End Uses}

Figure 24. Intensities for Natural Gas Water Heating and Cooking and for Electric Refrigeration, by Principal Building Activity, 1989

a. Natural Gas Water Heating

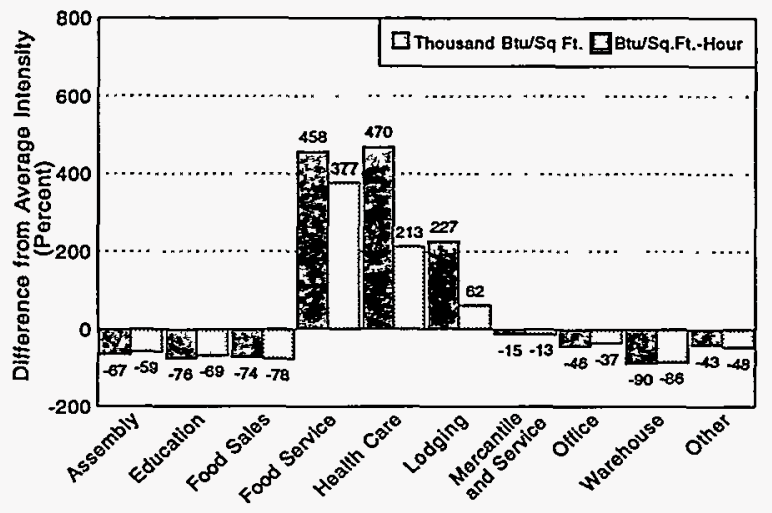

b. Natural Gas Cooking

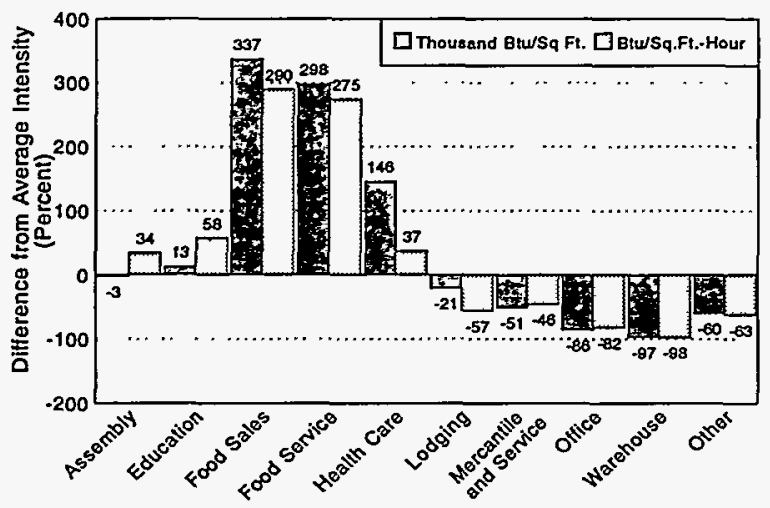

c. Electric Refrigeration

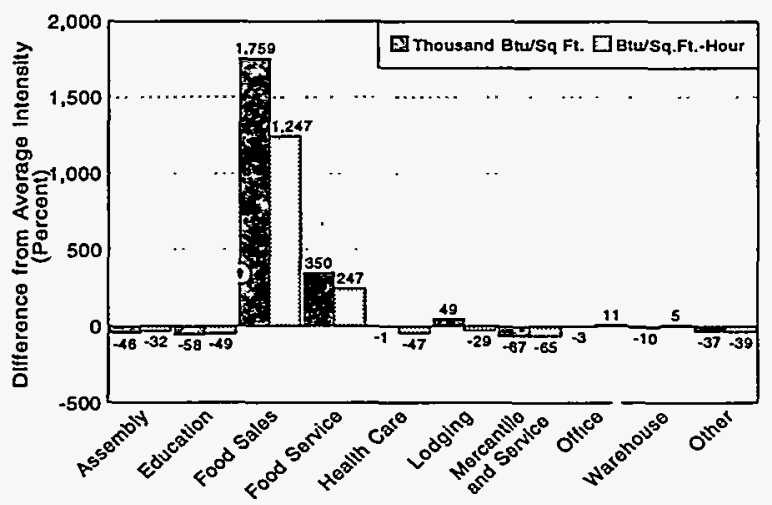

Sources: Energy Information Administration, Office of Energy Markets and End Use, Forms ElA-871A through F of the 1989 Commercial Buildings Energy Consumption Survey and statistically adjusted engineering end-use estimates.

- Figure 24 shows that food service and health care buildings had the highest water-heating intensities per square foot, more than five times the average for all buildings. Lodging buildings were the third most intensive, with a water-heating intensity three times the average for all buildings.

- Food sales and food service buildings had the highest energy intensities for cooking.

- Food-related buildings dominated the refrigeration intensities. Food sales buildings had the highest refrigeration intensities by far, nearly 20 times the average intensity per square foot of all commercial buildings. Food service buildings also had high refrigeration intensities, over four times the average intensity per square foot of all buildings. 
Figure 25. Electricity Intensities for Office Equipment, by Principal Building Activity and by Year Constructed, 1989

a. Principal Building Activity

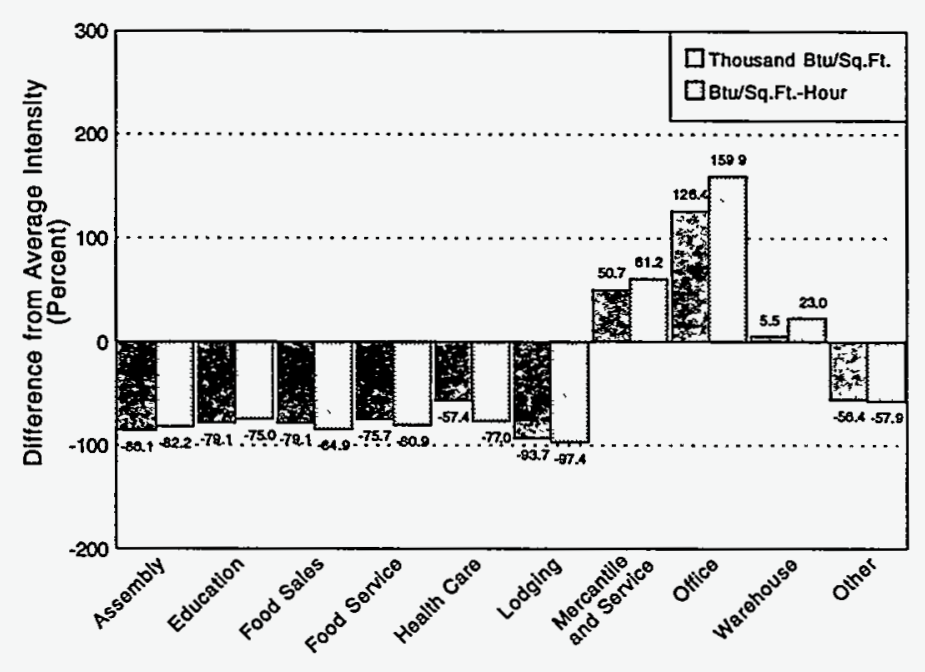

b. Year Constructed

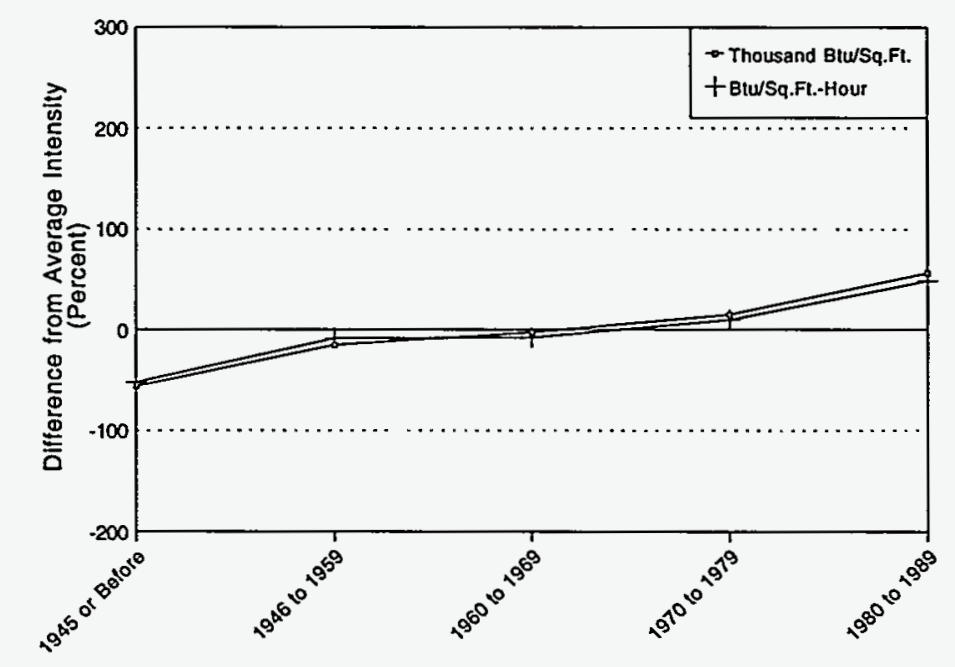

Sources: Energy Information Administration, Office of Energy Markets and End Use, Forms ElA-871A through F of the 1989 Commercial Buildings Energy Consumption Survey and statistically adjusted engineering end-use estimates.

- Figure 25 shows that office buildings had the highest energy intensities for office equipment.

- Mercantile and service buildings had the second highest intensities for office equipment.

- As with other electricity intensity patterns--such as cooling, ventilation, and lighting--the older buildings had relatively low intensities, with intensities increasing by vintage. Buildings constructed during the 1980's had the highest intensities. 


\section{Section 5. Targets for Reducing Energy Intensities}

This section identifies targets for reducing energy intensities in commercial buildings. These targets are energy end uses that would show large increases if all buildings consumed at the same rate as buildings of the 1980's. In this section, intensities are based upon the entire building stock, not just those buildings using a particular fuel for a given end use. This method of computing intensities reflects both the level of penetration and the efficiency of 1980's technologies in computing hypothetical consumption levels for the total building stock.

Key findings of this section include:

- If all buildings used natural gas for heating with the same intensity as did buildings constructed in the 1980's, the total consumption of natural gas for space heating would fall 201 trillion Btu, or 17 percent (Figure 26).

- If all buildings used electricity for cooling with the same intensity as did buildings constructed in the 1980's, the total consumption of electricity for cooling would drop by 23 trillion Btu. However, the consumption of electricity for ventilation would rise by 14 trillion Btu, for a net reduction of 9 trillion Btu, or 2 percent (Figure 27).

- If all buildings had the same lighting intensity as did buildings constructed in the 1980's, consumption of electricity for lighting would increase by 9 percent ( 94 trillion Btu) (Figure 28).

- If all buildings used office equipment with the same intensity as did 1980's buildings, consumption of electricity for office equipment would increase by 26 percent ( 99 trillion Btu) (Figure 29).

\section{Methodology}

The method used to determine targets for reducing energy intensities was to extrapolate the energy consumption patterns of the 1980's buildings to the entire commercial building stock. The method, performed for five major end uses (natural gas space heating, electric cooling, ventilation, lighting, and office equipment use), had two parts.

- First, the energy intensities of 1980 's buildings were calculated by using measures of energy intensities that accounted for differences in building size, operating hours, and weather (for heating and cooling). Energy intensities were calculated by principal building activity categories. For the five most numerous types of buildings in the CBECS sample (education, health care, mercantile, office, and warehouse), the buildings were divided into two groups: (1) small buildings, having 50,000 square feet of floorspace or less, and (2) large buildings, having over 50,000 square feet of floorspace.

- Second, estimates were made of the energy intensities and consumption for the five end uses, assuming that all buildings had the same end-use intensities as those of 1980's buildings.

The results show how the commercial building stock would be different if the entire commercial building stock consumed energy at the rates energy was consumed by the newer buildings, and also resembled the newer buildings in end uses served and in choices of energy sources for end uses. These results are based on the state of technology in commercial buildings in 1989 and do not account for any penetration of more efficient technologies (especially for lighting) which may have occurred since 1989. 


\section{Results for Selected End Uses}

Figure 26. Actual and Hypothetical Consumption of Natural Gas for Main Space Heating

a. Actual Intensity

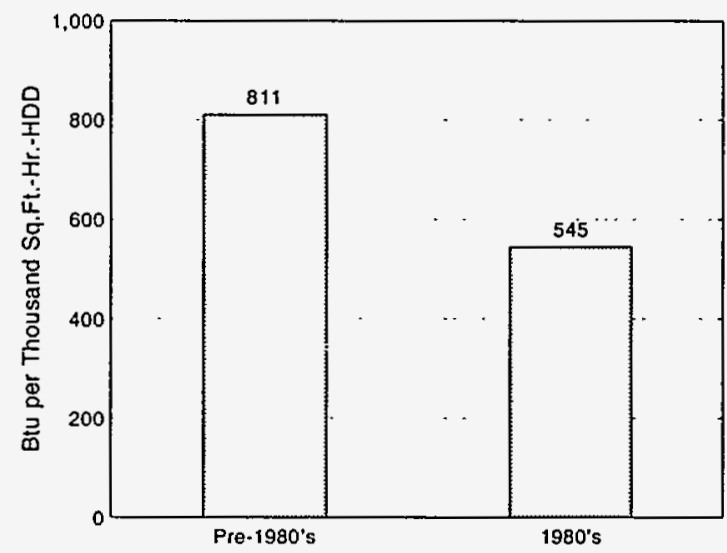

b. Consumption

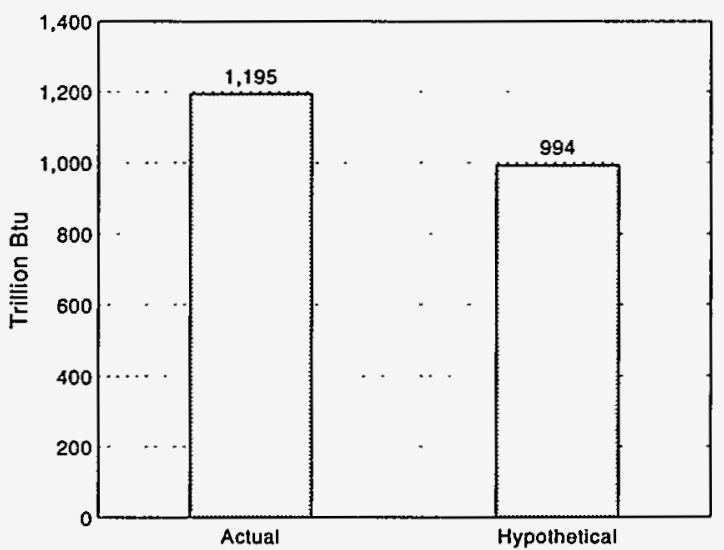

Note: Hypothetical consumption is that which would occur if all buildings consumed energy at the rate of 1980 's buildings.

Sources: Energy Information Administration, Office of Energy Markets and End Use, Forms ElA-871A through F of the 1989 Commercial Buildings Energy Consumption Survey and statistically adjusted engineering end-use estimates.

- Heating intensity can be expressed as the ratio of energy consumed for heating to the product of the number of square feet of heated floorspace, operating hours, and average daily HDD's. (This formula allows comparison of heating intensities in buildings of different sizes, with different operating hours, and in different climates.)

- Figure 26 shows that buildings constructed during the 1980's consumed natural gas for main space heating about a third less intensively (per heated floorspace-hour-degree-day) than did older buildings.

- If all buildings used natural gas for main space heating with the same intensity as did buildings constructed in the 1980's, the total consumption of natural gas for heating would fall 201 trillion Btu, or 17 percent of the actual total consumption for heating. 
Figure 27. Actual and Hypothetical Consumption of Electricity for Cooling and Ventilation

a. Actual Cooling Intensity

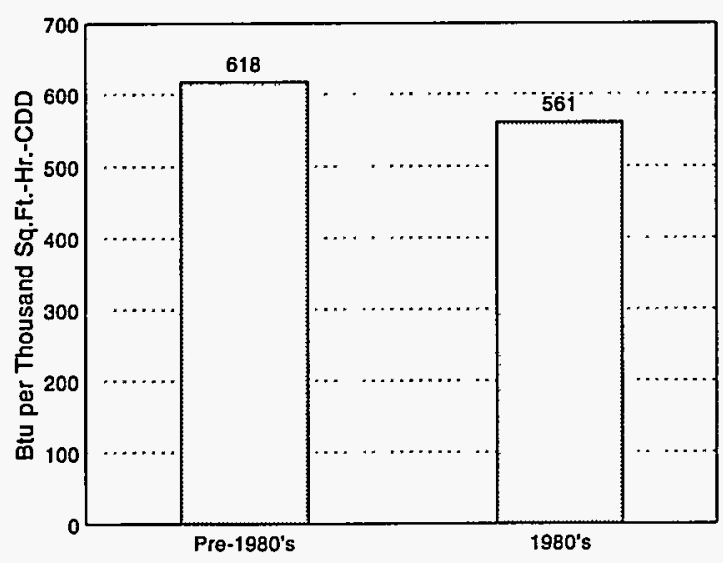

c. Actual Ventilation Intensity

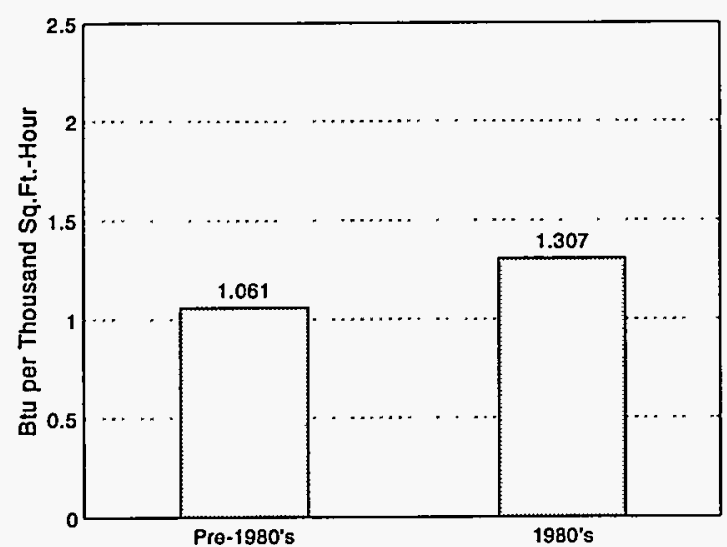

b. Consumption for Cooling

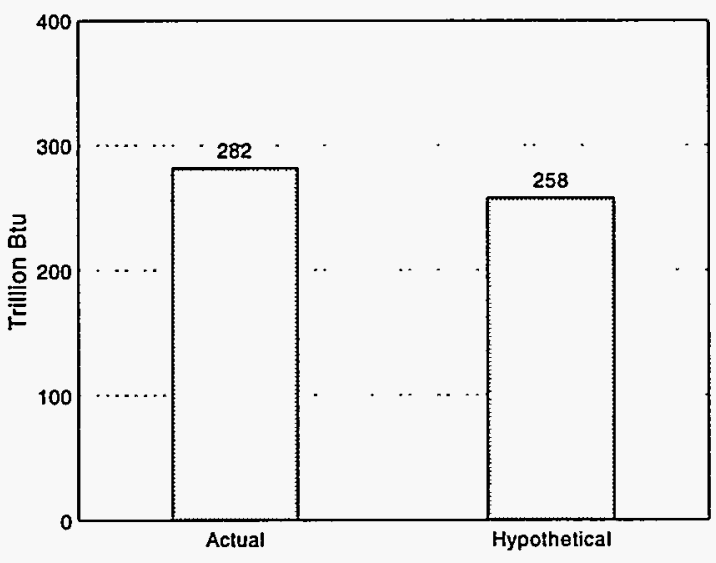

d. Consumption for Ventilation

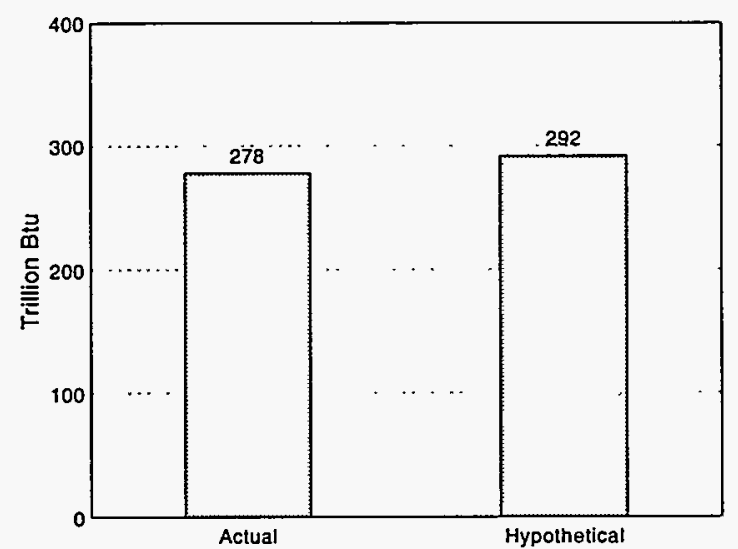

Note: Hypothetical consumption is that which would occur if all buildings consumed energy at the rate of 1980's buildings.

Sources: Energy Information Administration, Office of Energy Markets and End Use, Forms ElA-871A through F of the 1989 Commercial Buildings Energy Consumption Survey and statistically adjusted engineering end-use estimates.

- Cooling intensity can be expressed as the ratio of the energy consumed for cooling to the product of the number of square feet of cooled floorspace, operating hours, and average daily CDD's. (This formula allows comparison of cooling intensities in buildings of different sizes, with different operating hours, and in different climates.)

- Ventilation intensity can be expressed as the product of the number of square feet of ventilated floorspace and operating hours.

- Figure 27 shows that buildings constructed during the 1980's consumed electricity 9 percent less intensively for cooling, but 23 percent more intensively for ventilation, than did older buildings.

- If all buildings used electricity for cooling with the same intensity as buildings constructed in the 1980's, the total consumption of electricity for cooling would drop by 23 trillion Btu. However, the consumption of electricity for ventilation would rise by 14 trillion Btu, for a net reduction of 9 trillion Btu (2 percent). 
Figure 28. Actual and Hypothetical Consumption of Electricity for Lighting

a. Actual Intensity

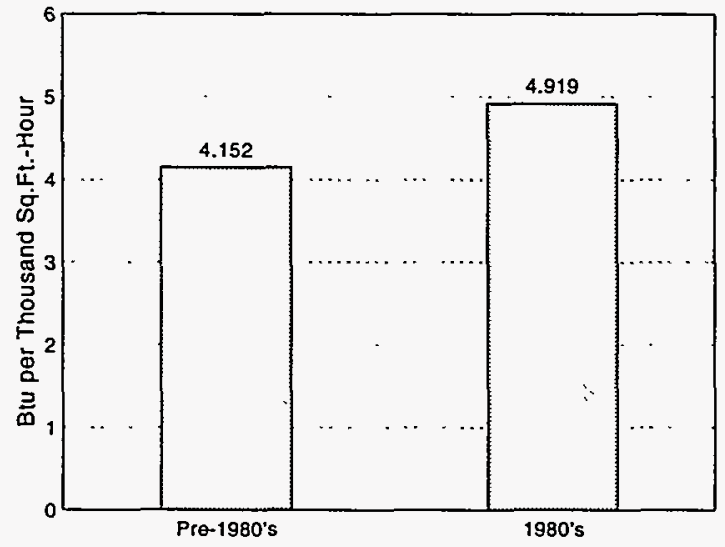

b. Consumption

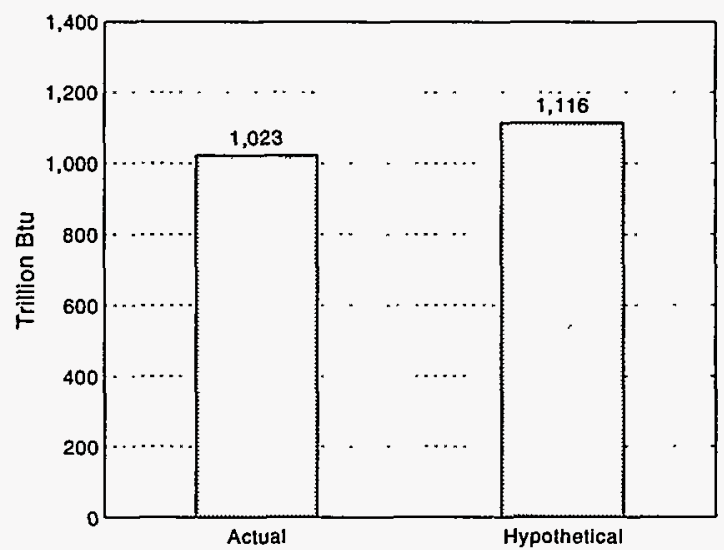

Note: Hypothetical consumption is that which would occur if all buildings consumed energy at the rate of 1980's buildings.

Sources: Energy Information Administration, Office of Energy Markets and End Use, Forms EIA-871A through F of the 1989 Commercial Buildings Energy Consumption Survey and statistically adjusted engineering end-use estimates.

- Lighting intensity can be expressed as the ratio of the energy consumed for lighting to the product of the number of square feet of floorspace lighted and operating hours.

- Figure 28 shows that if all buildings had the same lighting intensity as did buildings constructed in the 1980's, consumption of electricity for lighting would increase by 9 percent (94 trillion Btu). 
Figure 29. Actual and Hypothetical Consumption of Electricity for Office Equipment

a. Actual Intensity

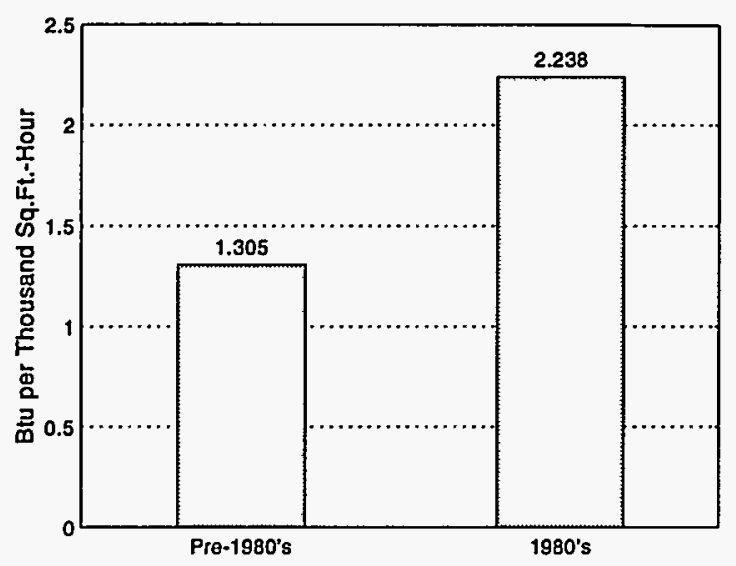

b. Consumption

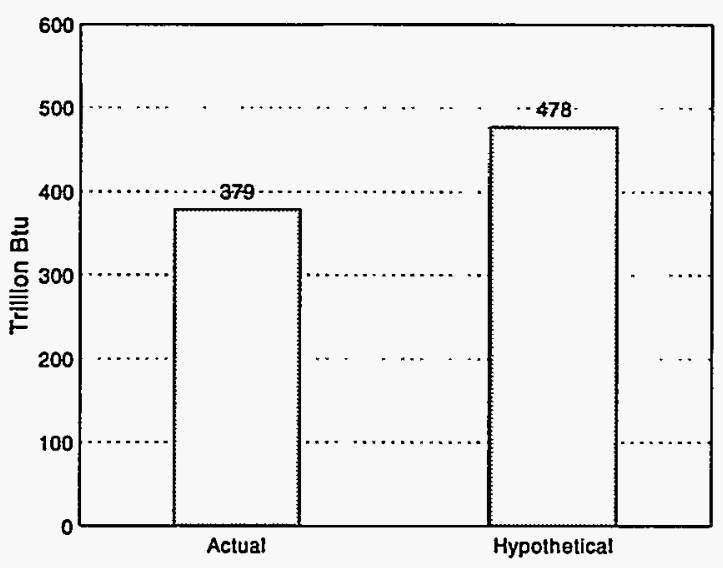

Note: Hypothetical consumption is that which would occur if all buildings consumed energy at the rate of 1980's buildings.

Sources: Energy Information Administration, Office of Energy Markets and End Use, Forms EIA-871A through F of the 1989 Commercial Buildings Energy Consumption Survey and statistically adjusted engineering end-use estimates.

- Energy intensity for office equipment can be expressed as the product of the square feet of floorspace and operating hours.

- Figure 29 shows that buildings constructed during the 1980's consumed electricity for office equipment much more intensively ( 71 percent more per floorspace-hour) than did older buildings.

- Applying the 1980 's intensities to all buildings, consumption of electricity for office equipment would increase by 26 percent ( 99 trillion Btu). 
Figure 30. Summary of Changes for Selected End-Use Energy Consumption and Expenditures that Would Occur If All Buildings Consumed at the Rate of 1980's Buildings

a. Change in Consumption (trillion Btu)

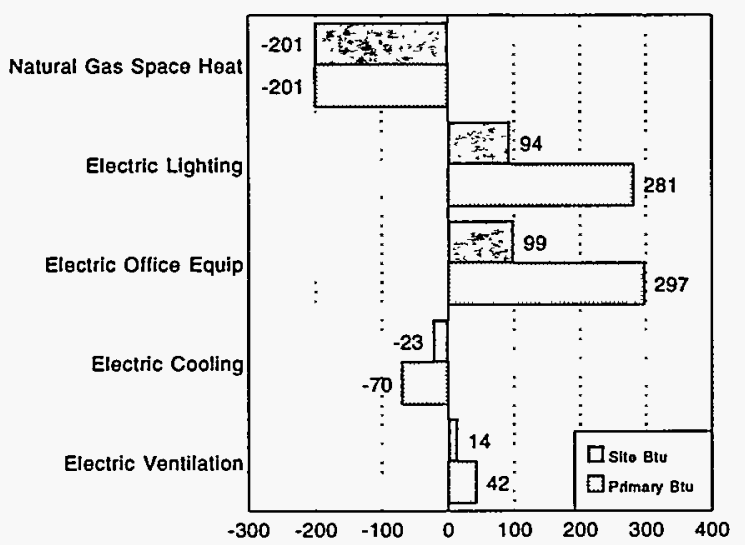

b. Change in Expenditures (million dollars)

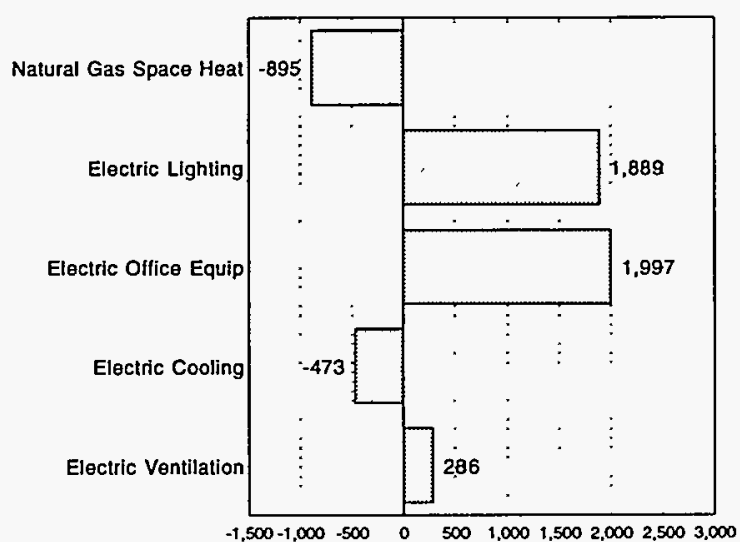

Sources: Energy Information Administration, Office of Energy Markets and End Use, Forms EIA-871A through F of the 1989 Commercial Buildings Energy Consumption Survey and statistically adjusted engineering end-use estimates.

- Figure 30 shows that, as the 1980 's buildings have shown, the demand for services is increasing, particularly in end uses served by electricity. It is important, for consumption in the 1990's and beyond, that these increasing demands be served by efficient technology.

- For the five selected end-uses, the changes in site energy consumption that would occur if all buildings consumed energy at the rate of the 1980's buildings would cancel each other out.

- The large decrease in natural gas consumption for space heating (201 trillion Btu) would be counterbalanced by increases in electricity consumption for lighting ( 94 trillion Btu) and office equipment (99 trillion Btu).

- Primary energy consumption would increase, driven by increases in intensity of electric lighting and office equipment use, despite gains in the efficiency of these technologies during the 1980's. Electricity end-use consumption would increase, offsetting decreases in fossil fuel end-use consumption.

- The increases in electricity consumption would have been greater if there had not been gains in the efficiency of technology during the 1980's. In particular, commercial lighting has been a prime target of commercial sector demand-side management programs sponsored by electric utilities, which were newly launched in 1989. 


\section{Appendix A \\ Development of End-Use Intensity Estimates}

The end-use estimates had two main sources: the 1989 Commercial Buildings Energy Consumption Survey (CBECS) and the Facility Energy Decision Screening (FEDS) system. The CBECS provided data on building characteristics and total energy consumption (i.e., for all end uses) for a national sample of commercial buildings. Using data collected by the CBECS, the FEDS engineering modules were used to produce estimates of energy consumption by end use. The FEDS engineering estimates were then statistically adjusted to match the CBECS total energy consumption.

This appendix briefly describes the 1989 CBECS, the FEDS load estimation methodology, the statistical adjustment procedure, and the remaining steps necessary to produce the final end-use estimates presented in this report.

\section{The Commercial Buildings Energy Consumption Survey}

The Energy Information Administration (EIA) is responsible for publishing national-level statistics on energy consumption by end users. Currently, the EIA publishes statistics for the residential, residential transportation (personal vehicles), commercial, and manufacturing sectors. For the commercial sector, consumption data are collected via a nationwide survey of commercial buildings, the CBECS.

The CBECS was designed to collect data on energy consumption, energy expenditures, and energy-related characteristics of commercial buildings. The sample consists of about 6,000 buildings, drawn from 119 locations nationwide. The survey is conducted in two stages: a Building Characteristics Survey and an Energy Suppliers Survey. The Building Characteristics Survey consists of personal interviews with knowledgeable respondents at each of the 6,000 buildings. The interview covers physical characteristics of the building, building occupancy patterns, major equipment, conservation practices, and the types and uses of energy in the building.

At the end of the interview, respondents are asked to provide the names and addresses of the companies that supply energy to their buildings in the form of electricity, natural gas, fuel oil, or district heating and cooling, and to sign a form authorizing the EIA to collect billing information directly from these energy supply companies. A separate mail survey, the Energy Suppliers Survey, asks these energy suppliers to provide data on the amounts and costs of energy delivered to the building during the survey year.

Additional details may be found in Appendix A, "How the Survey Was Conducted," of the two 1989 CBECS survey data reports.[3,4]

\section{The Facility Energy Decision Screening Engineering Estimates}

The energy consumption data provided by energy suppliers cover all end uses performed within commercial buildings. The total energy consumption can be disaggregated into end-use consumption by several approaches: engineering simulations, statistical modeling, or a hybrid approach known as statistically adjusted engineering (SAE). The 1989 CBECS end-use estimates were developed by using the SAE approach, with the FEDS system providing the initial engineering estimates.

The FEDS software was developed for the Department of Energy's Federal Energy Management Program (FEMP) and the U.S. Army Construction Engineering Research Laboratory as a tool for screening groups of buildings on 
federal facilities (such as Army bases) for energy efficiency retrofits.[2] The engineering modules, which estimate the energy load to be subjected to retrofit optimization, are the latest in a series of well-known building energy simulations, which include DOE-2 and ASEAM. In some ways FEDS is similar to Quick Input versions of ASEAM, in that it can operate from sparse data input. In other ways, such as its use of hourly load profiles rather than a bin method, FEDS is similar to DOE-2. The FEDS uses high-level installation information (number, age, size, and types of buildings and energy systems), an internal data base of typical energy-system configurations and performance data, and sophisticated energy simulation and optimization models to estimate the net present value of potential energy retrofits in federal installations. Release 1 of FEDS was used for the 1989 CBECS end-use estimates; Release 2 will be used for the 1992 CBECS end-use estimates.

The advantages of FEDS were that FEDS (without its retrofit optimization routines) can execute fairly quickly and that it does not demand a great deal of detailed data on each building. Both of these factors were important for the CBECS application, since there were nearly 6,000 buildings (with an average of two energy sources per building) and the only data available were those obtained from hour-long personal interviews.

The FEDS engineering models are designed to produce estimates for five end uses: space heating, cooling, ventilation, lighting, and water heating. Two other end uses, cooking and refrigeration, are also calculated internally by the model, although they are not part of the normal FEDS output. These seven end uses, plus an "other" end use, represent the FEDS accounting for total building end use. Estimates for office equipment energy use were not provided by the FEDS model.

Estimates for the first five end uses are based on detailed building engineering simulations. Estimates for the latter two are more sketchy and rely on parameters developed in the Regional End-Use Monitoring Program (REMP, formerly known as the End-Use Load and Consumer Assessment Program (ELCAP)) study.[6] REMP was a large end-use monitoring project sponsored by the Bonneville Power Administration. As designed to be used in facilities, only a general description of a building need be input for the building energy loads to be estimated interactively, relying on an extensive series of internal default values. Some of these defaults were based on data from the 1986 CBECS but many were based on REMP study. For use with the 1989 CBECS to produce the estimates in this report, the interface was changed from interactive to batch, with the 1989 CBECS data supplying as many values as possible.

Besides values relating to the building characteristics, the engineering estimates also required hourly weather profiles. For each calendar month, the average temperature during each hour of the day was calculated and input to the model.

\section{Statistically Adjusted Engineering Estimates}

The FEDS estimates were based on building characteristics and weather only. At the statistically adjusted engineering (SAE) stage, the consumption data were brought into play. The basic idea behind the SAE method is simple. Let $e u i_{b f u}$ be the end-use consumption per square foot estimated by the FEDS model for building $b$, fuel $f$, and end use $u$, and let $e u i_{b f}$ be the total energy consumption (from the CBECS Energy Suppliers Survey) per square foot for building $b$ and fuel $f$. Then a set of coefficients $a_{f u}$ can be estimated statistically, i.e., by multiple regression, such that

$$
e Q i_{b f}=\sum_{u} a_{f u} e u i_{b f u} .
$$

The coefficients adjust the FEDS engineering estimates upward or downward to match the reported energy use. The $e a i_{b f}$ are referred to as SAE estimates. 
In practice, this simple version of SAE proved difficult. Instead of following the simple model, energy use was partitioned into two parts, seasonal and nonseasonal. The two parts were then statistically adjusted separately.[1]

The SAE procedures were produced for aggregates of buildings by principal building activity. Only buildings with 12 months of reported 1989 billing data were used. The adjustments were performed separately for electricity and natural gas. Due to the limited number of cases, fuel oil and district heat SAE estimates were produced by using parameters estimated for natural gas.

\section{The Final End-Use Estimates}

Because the SAE procedure calibrated the engineering estimates to the reported data for aggregates of buildings, SAE estimates for individual buildings could still vary from the values on the 1989 CBECS Master File. For the final end-use estimates, those used in this report, the value on the CBECS Master File (whether reported or imputed) was prorated in proportion to the SAE estimates.

The office equipment estimate was also made after the SAE by using REMP estimates.[5] These estimates were based on data collected from mid-1986 to iate 1988 and so are roughly contemporaneous with the 1989 CBECS. The REMP estimates were used to estimate the office equipment share of the "other" end use energy consumption. Included in office equipment were large computer equipment (if the CBECS data indicated the presence of a computer area with a separate air-conditioning system), personal computer equipment, and general office equipment (typewriters, copiers, cash registers, etc.).

\section{The Calculation of Energy Intensities}

The energy intensities presented in this report for different sets of buildings are ratios of aggregate energy consumption to an aggregate measure of the demand for energy. The general equation was

$$
\text { Intensity }=\frac{\sum_{b} w_{b} e_{b}}{\sum_{b} w_{b} d_{b}},
$$

where $w_{b}$ is building $b$ 's weight (the number of buildings represented by building $b$ in the sample), $e_{b}$ is the amount of energy (total or end use) consumed by building $b$, and $d_{b}$ is the demand measure (discussed below) for building $b$. The quantities are summed for all buildings in a particular category before dividing.

For intensity per square foot, the demand $d_{b}$ is simply building $b$ 's total square footage. For more involved intensities, the measure of demand is calculated for each building before summing. For example, for intensity per square foot-hour, the demand $d_{b}$ calculated for each building $b$ is the product of the square footage and the annual operating hours for building $b$.

\section{References}

1. D. B. Belzer, L. E. Wrench, and T. L. Marsh, End-Use Energy Consumption Estimates for U. S. Commercial Buildings, 1989, PNL-8946 (Pacific Northwest Laboratory, Richland, WA, November 1993). 
2. J. A. Dirks and L. R. Wrench, "Federal Energy Decision Screening (FEDS) System Software," PNL-SA-22780 (Pacific Northwest Laboratory, Richland, WA, August 1993).

3. Energy Information Administration, Office of Energy Markets and End Use, Commercial Buildings Characteristics 1989, DOE/EIA-0246(89) (Washington, DC, June 1991).

4. Energy Information Administration, Office of Energy Markets and End Use, Commercial Buildings Energy Consumption and Expenditures 1989, DOE/EIA-0318(89) (Washington, DC, April 1992).

5. R. G. Pratt, M. A. Williamson, E. E. Richman, and N. E. Miller, Commercial Electric Loads: End-Use Load and Consumer Assessment Program (ELCAP), (Pacific Northwest Laboratory, Richland, WA, 1990).

6. Z. T. Taylor and R. G. Pratt, Description of Electric Energy Use in Commercial Buildings in the Pacific Northwest, DOE/BP-13795-22 (Pacific Northwest Laboratory, Richland, WA, 1989). 


\title{
Appendix B
}

\section{Detailed Tables}

\author{
Table Organization
}

The following 13 tables present detailed energy end-use consumption data from the 1989 CBECS. Summary tables for all major fuels (electricity, natural gas, fuel oil, and district heat) appear first, followed by separate tables for each of the four major fuels. Within each energy source's group of tables, there is a table showing end-use consumption, a table showing end-use intensities (consumption per square foot), and a table (except for fuel oil and district heat) showing the end-use shares of total consumption.

In the major fuels end-use intensity table, the intensity denominator is total commercial building floorspace. In the energy source-specific intensity tables, the intensity denominator is the total floorspace of all commercial buildings receiving that specific energy source.

\section{Row Stubs}

There is a standard set of row categories (stubs), which appears in all the summary tables. Depending on the specific table topic, the standard stub may be augmented with selected variables pertinent to that topic. The standard stub items always appear in the same order, with any additional stub items interspersed adjacent to the related standard stub items.

There are two types of row stubs, those that divide commercial buildings into exclusive, nonoverlapping categories and those that indicate nonexclusive, overlapping subsets. For example, "Climate Zone" is a set of exclusive categories; a given building belongs in only one of these. "Energy Sources," on the other hand, is a set of nonexclusive categories; a given building may be represented in more than one line under this stub, because the building may use more than one energy source. The phrase "Solely or in Combination" indicates that the categories under this row header are overlapping. Both exclusive and overlapping categories may be nonexhaustive; that is, there may be some buildings that do not fall into any of the listed categories.

\section{Relative Standard Errors}

Sampling error is inherent in any CBECS estimates, because the estimates are based on a relatively small sample randomly chosen to represent a large and variable population. Sampling errors are random differences between the survey estimate and the population value that occur because of the particular sample that was selected by chance. The average sampling error, averaged over all possible samples, would be zero. Although the sampling error is nonzero and unknown for the particular sample chosen, the sample design permits sampling errors to be estimated.

Due to the complexity of the sample design, the CBECS uses a jackknife replication method (with 40 collapsed strata) for variance estimation. To capture variation due to unit nonresponse, weight adjustment is performed separately within each replicate, as well as overall. The 40 sets of replicate weights are used to compute mean square errors about the full-sample point estimates.

The relative standard error (RSE) is the square root of the mean square error, expressed as a percent of the estimate. The RSE's which are displayed in the far-right columns of most tables are based only on the total energy 
consumption or intensity estimates only. No RSE estimates are provided for energy end-use estimates, since the enduse breakdown of total fuel consumption is completely modeled. For these derived estimates, the uncertainty of the engineering assumptions is generally greater than the sampling error. The RSE's for end-use energy are at least as large as that for the total fuel consumption. For more details about the derivation of the row and column RSE factors, see Appendix B, "Nonsampling and Sampling Errors," of the 1989 CBECS data reports.[1,2]

\section{References}

1. Energy Information Administration, Office of Energy Markets and End Use, Commercial Buildings Characteristics 1989, DOE/EIA-0246(89) (Washington, DC, June 1991).

2. Energy Information Administration, Office of Energy Markets and End Use, Commercial Buildings Energy Consumption and Expenditures 1989, DOE/EIA-0318(89) (Washington, DC, April 1992). 


\begin{tabular}{|c|c|c|c|c|c|c|c|c|c|c|c|}
\hline \multirow{3}{*}{$\begin{array}{c}\text { Building } \\
\text { Characteriatice } \\
\text { RSE Column Factor: }\end{array}$} & \multicolumn{10}{|c|}{$\begin{array}{c}\text { Sum of Major Fuel Consumption } \\
\text { (trillion Btu) }\end{array}$} & \multirow{3}{*}{$\begin{array}{l}\text { RSE } \\
\text { Row } \\
\text { Factor }\end{array}$} \\
\hline & \multirow[t]{2}{*}{ Total } & \multirow{2}{*}{$\begin{array}{c}\text { Space } \\
\text { Heating } \\
\text { NF }\end{array}$} & \multirow{2}{*}{$\begin{array}{c}\text { Cooling } \\
\text { NF }\end{array}$} & \multirow{2}{*}{$\begin{array}{c}\begin{array}{c}\text { Ventl- } \\
\text { ation }\end{array} \\
\text { NF }\end{array}$} & \multirow{2}{*}{$\begin{array}{c}\begin{array}{c}\text { Water } \\
\text { Heating }\end{array} \\
\mathrm{NF}\end{array}$} & \multirow{2}{*}{\begin{tabular}{|c|} 
Lighting \\
NF
\end{tabular}} & \multirow{2}{*}{\begin{tabular}{|c|} 
Cooking \\
NF
\end{tabular}} & \multirow{2}{*}{$\begin{array}{c}\begin{array}{l}\text { Retrig- } \\
\text { eration }\end{array} \\
\text { NF }\end{array}$} & \multirow{2}{*}{$\begin{array}{c}\text { Oftlce } \\
\text { Equip- } \\
\text { ment }\end{array}$} & \multirow{2}{*}{$\begin{array}{c}\text { Other } \\
\text { NF }\end{array}$} & \\
\hline & & & & & & & & & & & \\
\hline 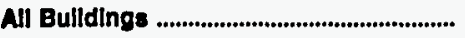 & 5,788 & 2,017 & 303 & 278 & 499 & 1,023 & 271 & 187 & 379 & 832 & 6.11 \\
\hline 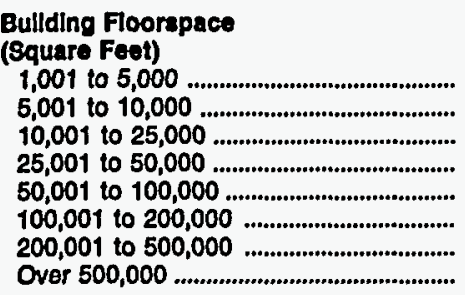 & $\begin{array}{l}692 \\
567 \\
791 \\
756 \\
855 \\
777 \\
698 \\
652\end{array}$ & $\begin{array}{l}256 \\
229 \\
327 \\
319 \\
299 \\
266 \\
200 \\
121\end{array}$ & $\begin{array}{l}44 \\
33 \\
40 \\
34 \\
37 \\
36 \\
36 \\
43\end{array}$ & $\begin{array}{l}29 \\
21 \\
27 \\
26 \\
40 \\
43 \\
50 \\
43\end{array}$ & $\begin{array}{l}76 \\
53 \\
57 \\
43 \\
70 \\
65 \\
57 \\
78\end{array}$ & $\begin{array}{r}96 \\
83 \\
124 \\
127 \\
161 \\
153 \\
151 \\
126\end{array}$ & $\begin{array}{l}50 \\
17 \\
20 \\
22 \\
24 \\
37 \\
22 \\
Q\end{array}$ & $\begin{array}{l}33 \\
16 \\
30 \\
27 \\
31 \\
19 \\
15 \\
15\end{array}$ & $\begin{array}{l}52 \\
41 \\
73 \\
46 \\
44 \\
47 \\
39 \\
37\end{array}$ & $\begin{array}{r}56 \\
75 \\
93 \\
113 \\
149 \\
109 \\
128 \\
109\end{array}$ & $\begin{array}{r}6.01 \\
9.94 \\
8.17 \\
10.13 \\
13.81 \\
13.60 \\
20.86 \\
27.21\end{array}$ \\
\hline 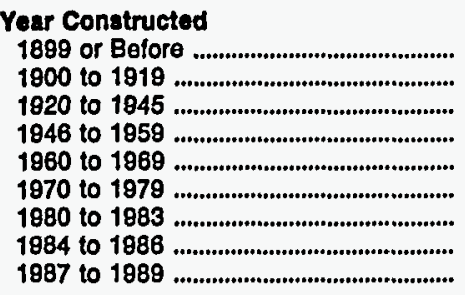 & $\begin{array}{r}128 \\
239 \\
636 \\
988 \\
1,275 \\
1,342 \\
432 \\
464 \\
284\end{array}$ & $\begin{array}{r}87 \\
111 \\
285 \\
395 \\
469 \\
399 \\
92 \\
102 \\
77\end{array}$ & $\begin{array}{r}2 \\
5 \\
26 \\
46 \\
65 \\
85 \\
31 \\
29 \\
15\end{array}$ & $\begin{array}{r}3 \\
7 \\
29 \\
42 \\
55 \\
70 \\
23 \\
37 \\
12\end{array}$ & $\begin{array}{r}12 \\
16 \\
64 \\
91 \\
115 \\
117 \\
26 \\
38 \\
21\end{array}$ & $\begin{array}{r}8 \\
29 \\
76 \\
158 \\
219 \\
276 \\
98 \\
104 \\
55\end{array}$ & $\begin{array}{r}4 \\
9 \\
28 \\
Q \\
38 \\
71 \\
17 \\
21 \\
13\end{array}$ & $\begin{array}{r}3 \\
4 \\
14 \\
21 \\
32 \\
51 \\
16 \\
25 \\
21\end{array}$ & $\begin{array}{r}3 \\
10 \\
23 \\
53 \\
72 \\
93 \\
47 \\
50 \\
28\end{array}$ & $\begin{array}{r}7 \\
Q \\
89 \\
114 \\
210 \\
179 \\
82 \\
59 \\
41\end{array}$ & $\begin{array}{r}19.55 \\
19.01 \\
19.00 \\
15.54 \\
11.41 \\
8.91 \\
14.15 \\
11.59 \\
20.61\end{array}$ \\
\hline $\begin{array}{l}\text { BUILDING USE } \\
\text { Principal Bullding Activity }\end{array}$ & & & & & & & & & & & \\
\hline 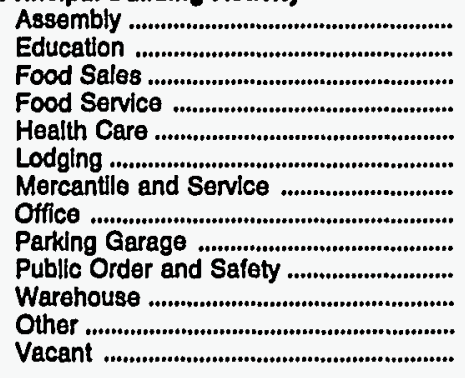 & $\begin{array}{r}441 \\
704 \\
139 \\
255 \\
449 \\
425 \\
1,048 \\
1,230 \\
42 \\
78 \\
536 \\
344 \\
98\end{array}$ & $\begin{array}{r}227 \\
377 \\
20 \\
48 \\
79 \\
137 \\
358 \\
363 \\
Q \\
42 \\
221 \\
86 \\
40\end{array}$ & $\begin{array}{r}25 \\
26 \\
16 \\
19 \\
33 \\
17 \\
53 \\
89 \\
\\
1 \\
6 \\
14 \\
3\end{array}$ & $\begin{array}{r}25 \\
24 \\
8 \\
13 \\
21 \\
20 \\
26 \\
133 \\
-2 \\
3 \\
1\end{array}$ & $\begin{array}{r}20 \\
40 \\
2 \\
49 \\
123 \\
125 \\
56 \\
47 \\
Q \\
Q \\
6 \\
15 \\
Q\end{array}$ & $\begin{array}{r}63 \\
114 \\
19 \\
27 \\
68 \\
47 \\
201 \\
272 \\
8 \\
13 \\
102 \\
76 \\
13\end{array}$ & $\begin{array}{c}18 \\
Q \\
21 \\
59 \\
45 \\
32 \\
23 \\
8 \\
Q \\
1 \\
1 \\
Q \\
1\end{array}$ & $\begin{array}{r}11 \\
10 \\
45 \\
16 \\
6 \\
16 \\
12 \\
35 \\
1 \\
2 \\
24 \\
7 \\
2\end{array}$ & $\begin{array}{r}6 \\
10 \\
1 \\
2 \\
5 \\
1 \\
115 \\
165 \\
1 \\
1 \\
57 \\
Q \\
2\end{array}$ & $\begin{array}{r}44 \\
49 \\
7 \\
21 \\
Q \\
31 \\
204 \\
117 \\
7 \\
11 \\
114 \\
126 \\
30\end{array}$ & $\begin{array}{r}12.63 \\
17.15 \\
21.52 \\
9.41 \\
25.01 \\
13.24 \\
10.23 \\
5.01 \\
25.95 \\
37.76 \\
18.24 \\
34.71 \\
29.15\end{array}$ \\
\hline 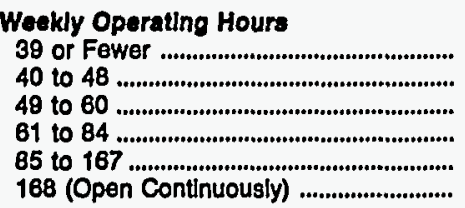 & $\begin{array}{r}203 \\
998 \\
925 \\
991 \\
998 \\
1,673\end{array}$ & $\begin{array}{l}121 \\
466 \\
371 \\
325 \\
324 \\
410\end{array}$ & $\begin{array}{l}7 \\
48 \\
46 \\
57 \\
58 \\
87\end{array}$ & $\begin{array}{l}10 \\
43 \\
52 \\
52 \\
38 \\
83\end{array}$ & $\begin{array}{r}10 \\
38 \\
32 \\
76 \\
62 \\
280\end{array}$ & $\begin{array}{r}23 \\
158 \\
169 \\
189 \\
186 \\
289\end{array}$ & $\begin{array}{r}3 \\
10 \\
15 \\
42 \\
101 \\
99\end{array}$ & $\begin{array}{r}6 \\
27 \\
24 \\
31 \\
44 \\
54\end{array}$ & $\begin{array}{l}7 \\
71 \\
93 \\
71 \\
56 \\
81\end{array}$ & $\begin{array}{r}17 \\
136 \\
122 \\
149 \\
119 \\
289\end{array}$ & $\begin{array}{r}8.37 \\
7.77 \\
6.94 \\
7.89 \\
15.24 \\
11.65\end{array}$ \\
\hline 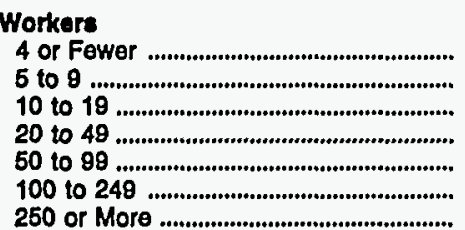 & $\begin{array}{r}697 \\
534 \\
540 \\
939 \\
701 \\
992 \\
1,386\end{array}$ & $\begin{array}{l}321 \\
205 \\
215 \\
395 \\
277 \\
321 \\
281\end{array}$ & $\begin{array}{l}34 \\
30 \\
30 \\
41 \\
37 \\
42 \\
89\end{array}$ & $\begin{array}{r}28 \\
21 \\
20 \\
27 \\
25 \\
43 \\
113\end{array}$ & $\begin{array}{r}68 \\
50 \\
47 \\
82 \\
28 \\
68 \\
155\end{array}$ & $\begin{array}{r}88 \\
83 \\
74 \\
156 \\
133 \\
201 \\
288\end{array}$ & $\begin{array}{r}23 \\
26 \\
26 \\
31 \\
21 \\
39 \\
105\end{array}$ & $\begin{array}{l}30 \\
17 \\
20 \\
33 \\
28 \\
29 \\
31\end{array}$ & $\begin{array}{l}44 \\
42 \\
42 \\
56 \\
39 \\
58 \\
99\end{array}$ & $\begin{array}{r}61 \\
61 \\
67 \\
117 \\
112 \\
190 \\
225\end{array}$ & $\begin{array}{r}4.94 \\
8.35 \\
7.34 \\
7.84 \\
11.18 \\
16.04 \\
18.30\end{array}$ \\
\hline
\end{tabular}

See footnotes at end of table. 
Table B1. Consumption of All Major Fuels by End Uses, 1989 (Continued)

\begin{tabular}{|c|c|c|c|c|c|c|c|c|c|c|c|}
\hline \multirow{3}{*}{$\begin{array}{c}\text { Bullding } \\
\text { Characteristlcs } \\
\text { RSE Column Factor: }\end{array}$} & \multicolumn{10}{|c|}{$\begin{array}{l}\text { Sum of Major Fuel Consumption } \\
\text { (trillion Btu) }\end{array}$} & \multirow{3}{*}{$\begin{array}{l}\text { RSE } \\
\text { Row } \\
\text { Factor }\end{array}$} \\
\hline & Total & $\begin{array}{c}\text { Space } \\
\text { Heating }\end{array}$ & Coollng & $\begin{array}{l}\text { Ventll- } \\
\text { atlon }\end{array}$ & $\begin{array}{c}\text { Water } \\
\text { Heating }\end{array}$ & Lighting & Cooking & $\begin{array}{l}\text { Refilg- } \\
\text { eration }\end{array}$ & $\begin{array}{c}\text { Ottlce } \\
\text { Equip- } \\
\text { ment }\end{array}$ & Other & \\
\hline & 1.0 & NF & NF & NF & NF & NF & NF & NF & NF & NF & \\
\hline \multicolumn{12}{|l|}{ Owhership and Occupancy } \\
\hline 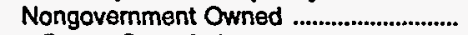 & 4,239 & 1,364 & 238 & 209 & 374 & 757 & 190 & 154 & 311 & 642 & 6.39 \\
\hline 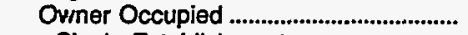 & 3,331 & 1,102 & 182 & 166 & 321 & 566 & 150 & 121 & 211 & 512 & 7.35 \\
\hline 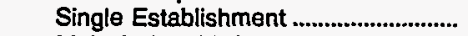 & 2,668 & 919 & 136 & 114 & 276 & 419 & 126 & 105 & 150 & 423 & 9.03 \\
\hline 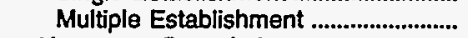 & 663 & 184 & 46 & 51 & 45 & 146 & 24 & 16 & 61 & 89 & 7.87 \\
\hline 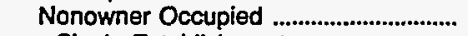 & 908 & 262 & 56 & 44 & 53 & 192 & 40 & 33 & 100 & 130 & 9.17 \\
\hline 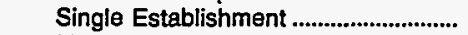 & 471 & 147 & 25 & 21 & 23 & 98 & 24 & 22 & 51 & 60 & 14.66 \\
\hline 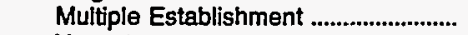 & 394 & 94 & 30 & 22 & 25 & 91 & 15 & 11 & 48 & 57 & 11.59 \\
\hline 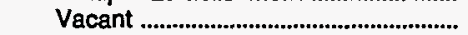 & $\mathbf{Q}$ & 21 & 1 & * & $\mathbf{Q}$ & 3 & $Q$ & . & . & $\ddot{a}$ & $\mathrm{NF}$ \\
\hline Government Owned ........................................ & 1,549 & 652 & 65 & 69 & 125 & 266 & 0 & 33 & 68 & 190 & 12.24 \\
\hline 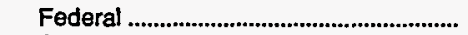 & Q & $\mathbf{Q}$ & $\mathbf{Q}$ & 17 & 11 & 60 & $Q$ & 5 & 16 & 28 & $\mathrm{NF}$ \\
\hline State & 585 & 255 & 20 & 22 & 56 & 83 & 13 & 14 & 29 & 83 & 20.97 \\
\hline Local & 692 & 327 & 27 & 31 & 58 & 122 & 20 & 15 & 23 & 69 & 11.73 \\
\hline \multicolumn{12}{|l|}{ Mutlbullding Facillty } \\
\hline Not on Multibuilding Facility ....................... & 2,887 & 1,055 & 153 & 146 & 219 & 510 & 116 & 114 & 213 & 361 & 4.76 \\
\hline $\begin{array}{l}\text { Part of Multibuilding Facility ........................... } \\
\text { On Facility with Central }\end{array}$ & 2,901 & 961 & 150 & 132 & 280 & 513 & 154 & 73 & 166 & 471 & 11.23 \\
\hline 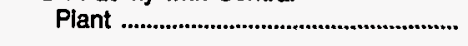 & 1,593 & 510 & 72 & 67 & 183 & 250 & 111 & 38 & 64 & 298 & 19.56 \\
\hline \multicolumn{12}{|l|}{$\begin{array}{l}\text { Percent Vacant at Least Three } \\
\text { Months }\end{array}$} \\
\hline 0 & 4,320 & 1,561 & 216 & 194 & 373 & 756 & 178 & 151 & 287 & 604 & 6.12 \\
\hline 1 to 50 & 1,086 & 302 & 69 & 71 & 95 & 217 & 39 & 25 & 80 & 188 & 12.86 \\
\hline 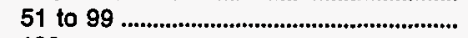 & 218 & 84 & $\mathbf{Q}$ & 7 & 11 & 28 & $\mathbf{Q}$ & 4 & 6 & 16 & 47.15 \\
\hline 100 & 164 & 70 & 6 & 6 & 21 & 21 & 3 & 6 & 6 & 24 & 17.52 \\
\hline \multicolumn{12}{|l|}{ Months In Use Out of Past 12 Months } \\
\hline 0 to 8 & 174 & 61 & 8 & 7 & 21 & 25 & 12 & 8 & 10 & 22 & 13.35 \\
\hline 9 to 11 & 272 & 162 & 7 & 11 & 22 & 32 & 3 & 4 & 4 & 26 & 14.63 \\
\hline 12 & 5,342 & 1,793 & 288 & 260 & 456 & 966 & 256 & 175 & 365 & 784 & 6.35 \\
\hline \multicolumn{12}{|l|}{$\begin{array}{l}\text { LOCATION } \\
\text { Census Reglon }\end{array}$} \\
\hline Northeast ........... & 1,354 & 567 & 42 & 57 & 115 & 227 & 47 & 35 & 92 & 172 & 11.10 \\
\hline 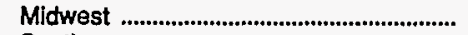 & 1,659 & 733 & 56 & 59 & 166 & 241 & 69 & 48 & 79 & 209 & 11.11 \\
\hline South & 1,648 & 423 & 136 & 93 & 113 & 341 & 63 & 65 & 122 & 291 & 11.08 \\
\hline West & 1,126 & 294 & 69 & 69 & 104 & 214 & $\mathbf{Q}$ & 38 & 86 & 159 & 12.85 \\
\hline Census Division & & & & & & & & & & & \\
\hline & & & & & & & & & & & \\
\hline 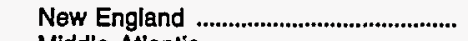 & 298 & 126 & 8 & 13 & 36 & 46 & 11 & 8 & 16 & 36 & 19.75 \\
\hline Middle Atlantic .......... & 1,056 & 441 & 34 & 44 & 80 & 182 & 36 & 28 & 76 & 137 & 13.34 \\
\hline Midwest & & & & & & & & & & & \\
\hline 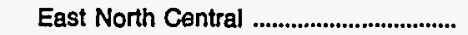 & 1,086 & 494 & 30 & 37 & 106 & 160 & 47 & 34 & 54 & 125 & 12.70 \\
\hline 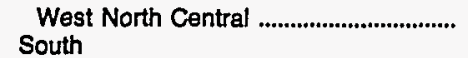 & 573 & 240 & 26 & 22 & 60 & 80 & 22 & 14 & 25 & 84 & 22.14 \\
\hline $\begin{array}{l}\text { South } \\
\text { South Atlantic ............................................... }\end{array}$ & 682 & 164 & 61 & 43 & 36 & 147 & 21 & 25 & & 130 & 16.43 \\
\hline 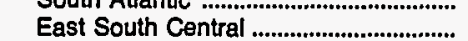 & 373 & 100 & 20 & 17 & 30 & 71 & 15 & 15 & 30 & 73 & 22.17 \\
\hline 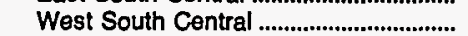 & 594 & 158 & 54 & 33 & 48 & 123 & 26 & 25 & 37 & 88 & 15.12 \\
\hline West & & & & & & & & & & & \\
\hline 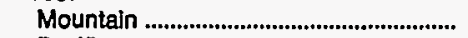 & 450 & 159 & 28 & 24 & 37 & 66 & $\mathbf{Q}$ & 12 & 24 & 38 & 28.59 \\
\hline 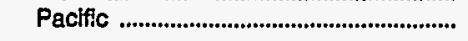 & 676 & 135 & 41 & 45 & 67 & 148 & 30 & 26 & 62 & 121 & 14.44 \\
\hline Motropolitan Status & & & & & & & & & & & \\
\hline 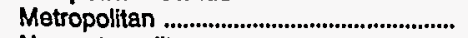 & 4,780 & 1,561 & 266 & 244 & 418 & 873 & 236 & 156 & 324 & 701 & 6.53 \\
\hline 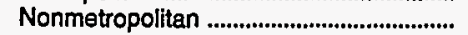 & 1,008 & 456 & 37 & 34 & 81 & 149 & 34 & 31 & 55 & 131 & 14.99 \\
\hline
\end{tabular}

See footnotes at end of table. 


\begin{tabular}{|c|c|c|c|c|c|c|c|c|c|c|c|}
\hline \multirow{3}{*}{$\begin{array}{c}\text { Building } \\
\text { Characteristics } \\
\text { RSE Column Factor: }\end{array}$} & \multicolumn{10}{|c|}{$\begin{array}{c}\text { Sum of Major Fuel Consumption } \\
\text { (trillion Btu) }\end{array}$} & \multirow{3}{*}{$\begin{array}{l}\text { RSE } \\
\text { Row } \\
\text { Factor }\end{array}$} \\
\hline & \multirow[t]{2}{*}{ Total } & \multirow{2}{*}{$\begin{array}{c}\text { Space } \\
\text { Heating } \\
\text { NF }\end{array}$} & \multirow{2}{*}{ Cooling } & \multirow{2}{*}{$\begin{array}{c}\begin{array}{c}\text { Ventil- } \\
\text { ation }\end{array} \\
\text { NF }\end{array}$} & \multirow{2}{*}{$\begin{array}{c}\begin{array}{c}\text { Water } \\
\text { Heating }\end{array} \\
\text { NF }\end{array}$} & \multirow{2}{*}{\begin{tabular}{|c|} 
Lghting \\
NF
\end{tabular}} & \multirow{2}{*}{$\begin{array}{c}\text { Cooking } \\
\text { NF }\end{array}$} & \multirow{2}{*}{$\begin{array}{l}\text { Refrig- } \\
\text { eration } \\
\text { NF }\end{array}$} & \multirow{2}{*}{$\begin{array}{c}\text { Offlce } \\
\text { Equip- } \\
\text { ment }\end{array}$} & \multirow{2}{*}{ Other } & \\
\hline & & & & & & & & & & & \\
\hline \multicolumn{12}{|l|}{$\begin{array}{l}\text { Climate Zone: 45-Year Average } \\
\text { Under 2,000 CDD and -- }\end{array}$} \\
\hline 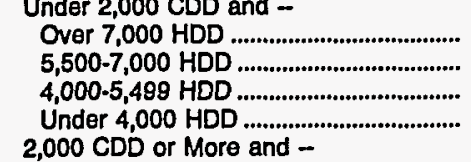 & $\begin{array}{r}617 \\
1,855 \\
1,393 \\
1,115\end{array}$ & $\begin{array}{l}298 \\
837 \\
452 \\
271\end{array}$ & $\begin{array}{l}10 \\
57 \\
67 \\
71\end{array}$ & $\begin{array}{l}16 \\
70 \\
72 \\
67\end{array}$ & $\begin{array}{r}67 \\
146 \\
128 \\
88\end{array}$ & $\begin{array}{r}84 \\
266 \\
279 \\
225\end{array}$ & $\begin{array}{r}18 \\
121 \\
52 \\
45\end{array}$ & $\begin{array}{l}23 \\
47 \\
42 \\
38\end{array}$ & $\begin{array}{r}30 \\
93 \\
102 \\
93\end{array}$ & $\begin{array}{r}70 \\
217 \\
198 \\
216\end{array}$ & $\begin{array}{l}20.74 \\
12.64 \\
13.62 \\
14.43\end{array}$ \\
\hline 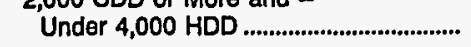 & 809 & 158 & 98 & 52 & 70 & 169 & 34 & 37 & 60 & 130 & 13.86 \\
\hline \multicolumn{12}{|l|}{$\begin{array}{l}1989 \text { Degree-Days } \\
\text { Under 2,000 CDD and -- }\end{array}$} \\
\hline 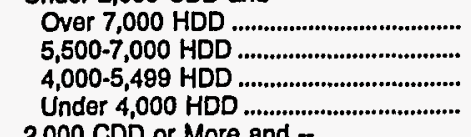 & $\begin{array}{r}875 \\
2,206 \\
917 \\
1,020\end{array}$ & $\begin{array}{l}448 \\
891 \\
280 \\
250\end{array}$ & $\begin{array}{l}14 \\
82 \\
45 \\
68\end{array}$ & $\begin{array}{l}23 \\
91 \\
54 \\
60\end{array}$ & $\begin{array}{r}78 \\
205 \\
69 \\
80\end{array}$ & $\begin{array}{l}117 \\
335 \\
212 \\
197\end{array}$ & $\begin{array}{r}30 \\
128 \\
41 \\
41\end{array}$ & $\begin{array}{l}29 \\
55 \\
34 \\
34\end{array}$ & $\begin{array}{r}38 \\
119 \\
81 \\
83\end{array}$ & $\begin{array}{r}98 \\
301 \\
102 \\
207\end{array}$ & $\begin{array}{l}18.16 \\
13.48 \\
10.88 \\
15.78\end{array}$ \\
\hline 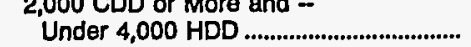 & 770 & 148 & 95 & 50 & 67 & 162 & 31 & 36 & 58 & 124 & 14.30 \\
\hline \multicolumn{12}{|l|}{$\begin{array}{l}\text { STRUCTURE } \\
\text { Floors }\end{array}$} \\
\hline $\begin{array}{l}1 \\
2 \\
4\end{array}$ & $\begin{array}{r}1,806 \\
1,532 \\
765 \\
893 \\
791\end{array}$ & $\begin{array}{l}637 \\
530 \\
340 \\
322 \\
187\end{array}$ & $\begin{array}{r}110 \\
70 \\
30 \\
45 \\
48\end{array}$ & $\begin{array}{l}71 \\
58 \\
35 \\
42 \\
73\end{array}$ & $\begin{array}{r}123 \\
94 \\
63 \\
111 \\
107\end{array}$ & $\begin{array}{l}322 \\
298 \\
107 \\
144 \\
151\end{array}$ & $\begin{array}{c}77 \\
44 \\
18 \\
Q \\
46\end{array}$ & $\begin{array}{l}74 \\
59 \\
15 \\
20 \\
19\end{array}$ & $\begin{array}{r}142 \\
114 \\
38 \\
35 \\
51\end{array}$ & $\begin{array}{r}251 \\
264 \\
120 \\
88 \\
109\end{array}$ & $\begin{array}{r}7.09 \\
9.40 \\
12.93 \\
17.64 \\
18.31\end{array}$ \\
\hline \multicolumn{12}{|l|}{ Wall Materials } \\
\hline 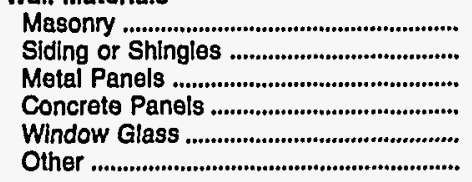 & $\begin{array}{r}3,919 \\
325 \\
457 \\
706 \\
224 \\
156\end{array}$ & $\begin{array}{r}1,466 \\
118 \\
162 \\
172 \\
53 \\
46\end{array}$ & $\begin{array}{r}200 \\
15 \\
21 \\
46 \\
13 \\
7\end{array}$ & $\begin{array}{r}168 \\
12 \\
19 \\
38 \\
26 \\
14\end{array}$ & $\begin{array}{r}381 \\
37 \\
18 \\
36 \\
17 \\
9\end{array}$ & $\begin{array}{r}634 \\
56 \\
97 \\
144 \\
54 \\
39\end{array}$ & $\begin{array}{r}174 \\
14 \\
6 \\
Q \\
Q \\
3\end{array}$ & $\begin{array}{r}127 \\
10 \\
15 \\
24 \\
7 \\
4\end{array}$ & $\begin{array}{r}230 \\
22 \\
42 \\
46 \\
24 \\
15\end{array}$ & $\begin{array}{r}539 \\
40 \\
79 \\
134 \\
20 \\
20\end{array}$ & $\begin{array}{r}6.15 \\
9.21 \\
23.07 \\
20.16 \\
21.66 \\
20.34\end{array}$ \\
\hline \multicolumn{12}{|l|}{ Roof Materials } \\
\hline 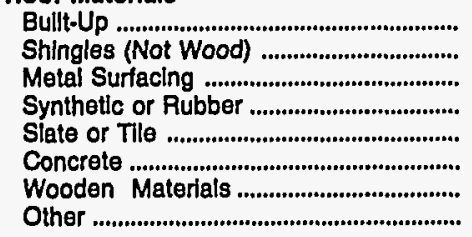 & $\begin{array}{r}3,019 \\
794 \\
597 \\
850 \\
206 \\
111 \\
63 \\
Q\end{array}$ & $\begin{array}{r}1,019 \\
327 \\
218 \\
288 \\
95 \\
20 \\
24 \\
27\end{array}$ & $\begin{array}{r}172 \\
40 \\
21 \\
42 \\
8 \\
8 \\
4 \\
0\end{array}$ & $\begin{array}{r}152 \\
35 \\
17 \\
45 \\
8 \\
12 \\
4 \\
6\end{array}$ & $\begin{array}{r}246 \\
76 \\
23 \\
77 \\
20 \\
14 \\
7 \\
0\end{array}$ & $\begin{array}{r}556 \\
119 \\
118 \\
151 \\
23 \\
28 \\
9 \\
19\end{array}$ & $\begin{array}{r}155 \\
36 \\
12 \\
30 \\
19 \\
Q \\
4 \\
Q\end{array}$ & $\begin{array}{r}92 \\
31 \\
20 \\
30 \\
6 \\
3 \\
2 \\
2\end{array}$ & $\begin{array}{r}191 \\
45 \\
59 \\
57 \\
Q \\
10 \\
3 \\
5\end{array}$ & $\begin{array}{r}435 \\
86 \\
107 \\
131 \\
19 \\
11 \\
7 \\
Q\end{array}$ & $\begin{array}{r}7.81 \\
9.59 \\
17.08 \\
15.49 \\
15.71 \\
29.02 \\
17.97 \\
\text { NF }\end{array}$ \\
\hline \multicolumn{12}{|l|}{$\begin{array}{l}\text { Buliding Shell Conservation } \\
\text { Features (Solely or In Combination) }\end{array}$} \\
\hline $\begin{array}{l}\text { Roof or Celling Insulation ....................... } \\
\text { Wall Insulation .......................................... } \\
\text { Storm or Multiple Glazing ........................ } \\
\text { Tlnted, Reflective, or Shading }\end{array}$ & $\begin{array}{l}4,486 \\
3,056 \\
2,557\end{array}$ & $\begin{array}{r}1,500 \\
941 \\
848\end{array}$ & $\begin{array}{l}252 \\
176 \\
130\end{array}$ & $\begin{array}{l}223 \\
148 \\
128\end{array}$ & $\begin{array}{l}407 \\
272 \\
287\end{array}$ & $\begin{array}{l}820 \\
585 \\
455\end{array}$ & $\begin{array}{l}221 \\
166 \\
126\end{array}$ & $\begin{array}{r}149 \\
105 \\
92\end{array}$ & $\begin{array}{l}306 \\
216 \\
180\end{array}$ & $\begin{array}{l}608 \\
445 \\
310\end{array}$ & $\begin{array}{l}7.23 \\
9.25 \\
7.91\end{array}$ \\
\hline 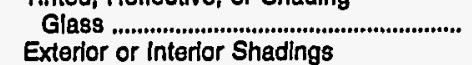 & 2,385 & 657 & 150 & 143 & 193 & 490 & 135 & 76 & 189 & 352 & 9.87 \\
\hline $\begin{array}{l}\text { or Awnings ......................................... } \\
\text { Weather Stripping or Caulking ............... } \\
\text { None of the Above .................................. }\end{array}$ & $\begin{array}{r}2,720 \\
4,549 \\
335\end{array}$ & $\begin{array}{r}848 \\
1,529 \\
126\end{array}$ & $\begin{array}{r}158 \\
251 \\
12\end{array}$ & $\begin{array}{r}149 \\
229 \\
12\end{array}$ & $\begin{array}{r}230 \\
404 \\
28\end{array}$ & $\begin{array}{r}496 \\
829 \\
51\end{array}$ & $\begin{array}{r}162 \\
233 \\
12\end{array}$ & $\begin{array}{r}82 \\
145 \\
11\end{array}$ & $\begin{array}{r}195 \\
310 \\
19\end{array}$ & $\begin{array}{r}399 \\
621 \\
64\end{array}$ & $\begin{array}{r}9.18 \\
6.66 \\
15.41\end{array}$ \\
\hline
\end{tabular}

See footnotes at end of table. 
Table B1. Consumption of All Major Fuels by End Use, 1989 (Continued)

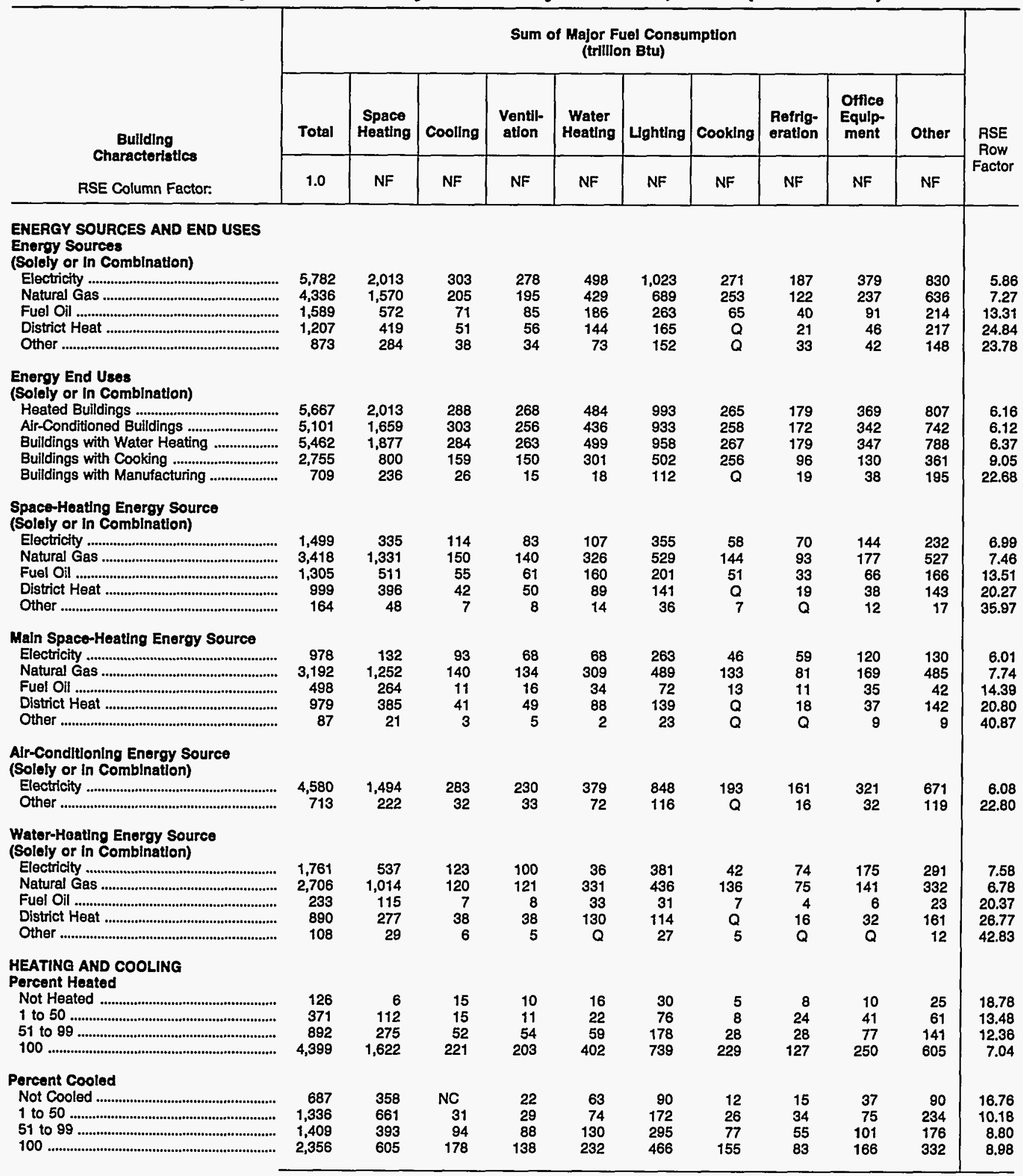

See footnotes at end of table. 


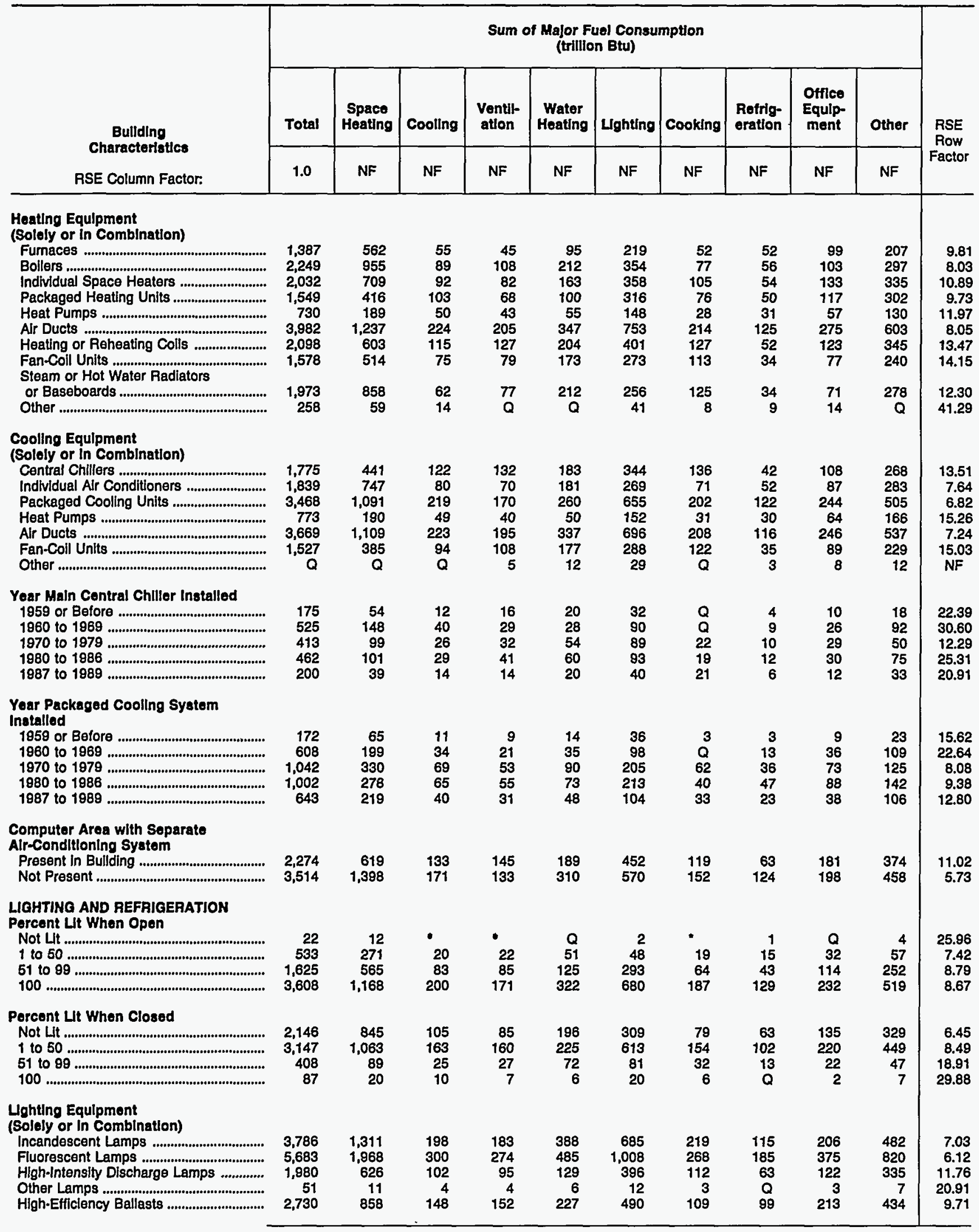

See footnotes at end of table. 
Table B1. Consumption of All Major Fuels by End Use, 1989 (Continued)

\begin{tabular}{|c|c|c|c|c|c|c|c|c|c|c|c|}
\hline \multirow{3}{*}{$\begin{array}{c}\text { Bullding } \\
\text { Characteristics } \\
\text { RSE Column Factor: }\end{array}$} & \multicolumn{10}{|c|}{$\begin{array}{l}\text { Sum of Major Fuel Consumption } \\
\text { (trillion Btu) }\end{array}$} & \multirow{3}{*}{$\begin{array}{l}\text { RSE } \\
\text { Row } \\
\text { Factor }\end{array}$} \\
\hline & Total & $\begin{array}{c}\text { Space } \\
\text { Heating }\end{array}$ & Cooling & $\begin{array}{l}\text { Ventll- } \\
\text { ation }\end{array}$ & $\begin{array}{l}\text { Water } \\
\text { Heating }\end{array}$ & Lghting & Cooking & $\begin{array}{l}\text { Retrig- } \\
\text { eration }\end{array}$ & $\begin{array}{l}\text { Offlice } \\
\text { Equlp- } \\
\text { ment }\end{array}$ & Other & \\
\hline & 1.0 & NF & NF & NF & NF & NF & NF & NF & NF & NF & \\
\hline \multicolumn{12}{|l|}{$\begin{array}{l}\text { Refrigeration Equlpment } \\
\text { (Solely or in Combination) } \\
\text { Commercial }\end{array}$} \\
\hline 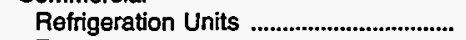 & 2,974 & 832 & 174 & 160 & 318 & 552 & 251 & 128 & 158 & 401 & 8.57 \\
\hline 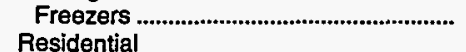 & 2,802 & 750 & 167 & 150 & 309 & 526 & 244 & 124 & 148 & 383 & 8.70 \\
\hline 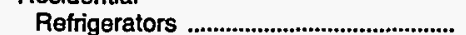 & 4,411 & 1,551 & 222 & 215 & 382 & 794 & 189 & 117 & 292 & 649 & 7.40 \\
\hline Freezers ............................................ & 1,479 & 448 & 84 & 63 & 172 & 248 & 129 & 42 & 65 & 227 & 13.72 \\
\hline 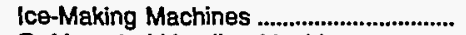 & 2,988 & 757 & 192 & 177 & 340 & 562 & 242 & 111 & 164 & 444 & 8.67 \\
\hline Refrigerated Vending Machines ............... & 4,347 & 1,388 & 234 & 222 & 391 & 816 & 224 & 141 & 283 & 651 & 7.38 \\
\hline 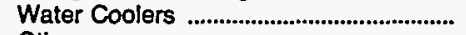 & 4,454 & 1,513 & 236 & 224 & 365 & 811 & 193 & 124 & 289 & 699 & 8.01 \\
\hline Other & 346 & 59 & 22 & 14 & $\mathbf{Q}$ & 55 & 17 & 10 & 22 & 103 & 35.63 \\
\hline \multicolumn{12}{|l|}{$\begin{array}{l}\text { ENERGY MANAGEMENT } \\
\text { Occupant Control }\end{array}$} \\
\hline 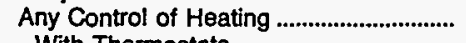 & 2,331 & 805 & 126 & 97 & 251 & 393 & 110 & 67 & 147 & 334 & 7.04 \\
\hline With Thermostats & 2,158 & 739 & 115 & 89 & 238 & 362 & 100 & 58 & 135 & 321 & 7.84 \\
\hline 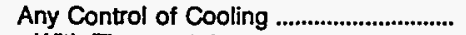 & 2,320 & 783 & 137 & 100 & 248 & 394 & 112 & 68 & 148 & 329 & 7.17 \\
\hline 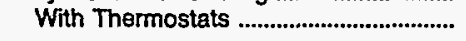 & 2,115 & 691 & 127 & 91 & 225 & 363 & 105 & 62 & 135 & 315 & 8.28 \\
\hline \multicolumn{12}{|l|}{ Reduced Use During Off-Hours } \\
\hline 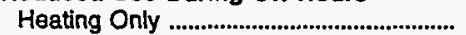 & 649 & 333 & 9 & 21 & 60 & 85 & 16 & 15 & 33 & 78 & 16.76 \\
\hline 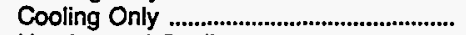 & 429 & 119 & 26 & 18 & 28 & 69 & 21 & 11 & 30 & 106 & 14.79 \\
\hline 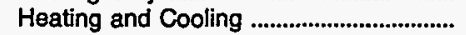 & 3,347 & 1,173 & 190 & 178 & 229 & 616 & 161 & 98 & 233 & 467 & 7.42 \\
\hline \multicolumn{12}{|l|}{$\begin{array}{l}\text { Computerized Energy Management } \\
\text { and Control System }\end{array}$} \\
\hline 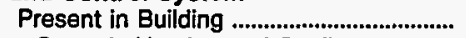 & 1,714 & 458 & 109 & 115 & 164 & 356 & 108 & 56 & 99 & 248 & 12.73 \\
\hline Controls Heating and Cooling ................ & 1,668 & 453 & 107 & 113 & 159 & 344 & 104 & 53 & 86 & 239 & 13.31 \\
\hline 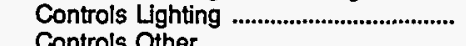 & 431 & 96 & 30 & 22 & 20 & 88 & 0 & 16 & 26 & 70 & 29.23 \\
\hline Controls Other ..... & 336 & 67 & 23 & 19 & $\mathbf{Q}$ & 62 & 14 & 10 & 16 & 74 & 35.31 \\
\hline \multicolumn{12}{|l|}{ Other Energy Management } \\
\hline Regular HVAC Maintenance ...................... & 4,773 & 1,594 & 255 & 241 & 413 & 870 & 221 & 153 & 307 & 717 & 6.86 \\
\hline 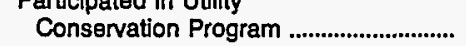 & 1,206 & 412 & 66 & 75 & 115 & 214 & 53 & 40 & 71 & 160 & 9.79 \\
\hline
\end{tabular}

$-=$ Value rounds to zero in the units displayed.

$\mathrm{NC}=$ No cases in responding sample.

$\mathrm{NF}=$ No applicable RSE row/column factor.

$Q=$ Data withheld because the Relative Standard Error (RSE) was greater than 50 percent, or data were reported for fewer than 20 bulldings.

Notes: - To obtain the RSE percentage for any table cell, multiply the corresponding RSE column and RSE row factors. - See Glossary for explanation of abbreviations and definitions of terms used in this report. - Because of rounding, date may not sum to totals.

Source: Energy Information Administration, Office of Energy Markets and End Use, Forms EIA-871A through F of the 1989 Commercial Buildings Energy Consumption Survey. 


\begin{tabular}{|c|c|c|c|c|c|c|c|c|c|c|c|}
\hline \multirow{3}{*}{$\begin{array}{c}\text { Bullding } \\
\text { Characteristics } \\
\text { RSE Column Factor: }\end{array}$} & \multicolumn{10}{|c|}{$\begin{array}{l}\text { Energy Intensity for Major Fuels } \\
\text { (thousand Btu per sq. ft.) }\end{array}$} & \multirow{3}{*}{$\begin{array}{l}\text { RSE } \\
\text { Row } \\
\text { Factor }\end{array}$} \\
\hline & Total & $\begin{array}{l}\text { Space } \\
\text { Heating }\end{array}$ & Cooling & $\begin{array}{l}\text { Ventil- } \\
\text { ation }\end{array}$ & $\begin{array}{c}\text { Water } \\
\text { Heating }\end{array}$ & Llghtling & Cooking & $\begin{array}{l}\text { Refrig- } \\
\text { eration }\end{array}$ & $\begin{array}{l}\text { Office } \\
\text { Equip- } \\
\text { ment }\end{array}$ & Other & \\
\hline & 1.0 & NF & NF & NF & NF & NF & NF & NF & NF & NF & \\
\hline All Bulldings & 91.6 & 31.9 & 4.8 & 4.4 & 7.9 & 16.2 & 4.3 & 3.0 & 6.0 & 13.2 & 4.95 \\
\hline 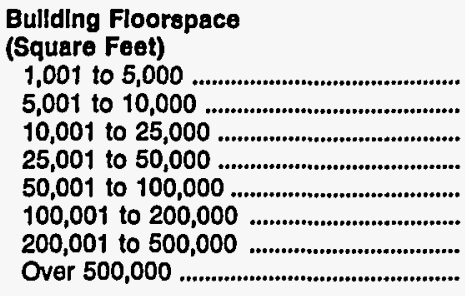 & $\begin{array}{r}101.9 \\
86.8 \\
76.1 \\
85.9 \\
93.7 \\
93.9 \\
99.4 \\
104.5\end{array}$ & $\begin{array}{l}37.7 \\
35.1 \\
31.5 \\
36.2 \\
32.8 \\
32.2 \\
28.5 \\
19.4\end{array}$ & $\begin{array}{l}6.5 \\
5.0 \\
3.9 \\
3.9 \\
4.0 \\
4.4 \\
5.1 \\
6.9\end{array}$ & $\begin{array}{l}4.2 \\
3.2 \\
2.6 \\
2.9 \\
4.3 \\
5.2 \\
7.1 \\
6.9\end{array}$ & $\begin{array}{r}11.1 \\
8.1 \\
5.5 \\
4.8 \\
7.7 \\
7.9 \\
8.1 \\
12.6\end{array}$ & $\begin{array}{l}14.2 \\
12.7 \\
12.0 \\
14.4 \\
17.7 \\
18.5 \\
21.5 \\
20.2\end{array}$ & $\begin{array}{l}7.3 \\
2.6 \\
2.0 \\
2.5 \\
2.7 \\
4.5 \\
3.1 \\
0\end{array}$ & $\begin{array}{l}4.9 \\
2.5 \\
2.9 \\
3.1 \\
3.4 \\
2.3 \\
2.2 \\
2.5\end{array}$ & $\begin{array}{l}7.7 \\
6.3 \\
7.0 \\
5.2 \\
4.9 \\
5.6 \\
5.6 \\
6.0\end{array}$ & $\begin{array}{r}8.3 \\
11.4 \\
8.9 \\
12.9 \\
16.3 \\
13.2 \\
18.2 \\
17.5\end{array}$ & $\begin{array}{r}4.76 \\
8.36 \\
5.88 \\
8.54 \\
12.68 \\
9.73 \\
16.68 \\
20.20\end{array}$ \\
\hline 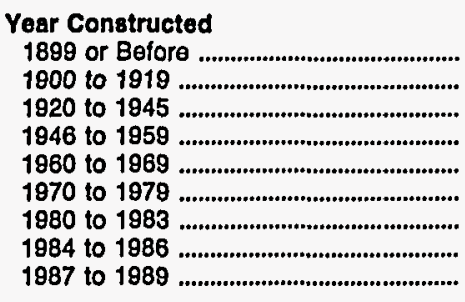 & $\begin{array}{r}77.2 \\
56.4 \\
78.5 \\
94.0 \\
104.8 \\
100.7 \\
101.2 \\
81.9 \\
87.7\end{array}$ & $\begin{array}{l}52.7 \\
26.1 \\
35.2 \\
37.5 \\
38.6 \\
29.9 \\
21.5 \\
18.0 \\
23.7\end{array}$ & $\begin{array}{l}1.1 \\
1.2 \\
3.2 \\
4.4 \\
5.3 \\
6.4 \\
7.3 \\
5.1 \\
4.5\end{array}$ & $\begin{array}{l}1.5 \\
1.5 \\
3.6 \\
4.0 \\
4.5 \\
5.3 \\
5.3 \\
6.5 \\
3.8\end{array}$ & $\begin{array}{l}7.2 \\
3.7 \\
7.9 \\
8.6 \\
9.5 \\
8.8 \\
6.1 \\
6.6 \\
6.4\end{array}$ & $\begin{array}{r}5.1 \\
6.7 \\
9.4 \\
15.0 \\
18.0 \\
20.7 \\
22.9 \\
18.3 \\
17.1\end{array}$ & $\begin{array}{r}2.2 \\
2.0 \\
3.5 \\
Q \\
3.1 \\
5.3 \\
4.0 \\
3.7 \\
4.1\end{array}$ & $\begin{array}{r}1.5 \\
.9 \\
1.7 \\
2.0 \\
2.7 \\
3.9 \\
3.8 \\
4.5 \\
6.5\end{array}$ & $\begin{array}{r}1.8 \\
2.3 \\
2.9 \\
5.1 \\
5.9 \\
7.0 \\
10.9 \\
8.9 \\
8.7\end{array}$ & $\begin{array}{r}4.0 \\
11.9 \\
11.0 \\
10.8 \\
17.3 \\
13.4 \\
19.3 \\
10.3 \\
12.8\end{array}$ & $\begin{array}{r}12.85 \\
25.02 \\
15.75 \\
11.13 \\
10.07 \\
7.18 \\
9.80 \\
10.75 \\
16.22\end{array}$ \\
\hline 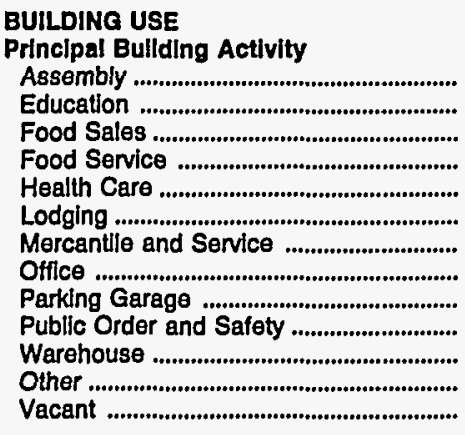 & $\begin{array}{r}63.8 \\
87.2 \\
175.6 \\
218.4 \\
218.7 \\
122.4 \\
84.8 \\
104.2 \\
42.6 \\
127.0 \\
57.9 \\
224.8 \\
23.5\end{array}$ & $\begin{array}{r}32.9 \\
46.7 \\
25.5 \\
41.5 \\
38.3 \\
39.3 \\
28.9 \\
30.7 \\
19.9 \\
67.6 \\
23.9 \\
56.0 \\
9.6\end{array}$ & $\begin{array}{r}3.7 \\
3.2 \\
20.0 \\
16.5 \\
16.1 \\
4.9 \\
4.3 \\
7.5 \\
.4 \\
2.3 \\
.6 \\
9.4 \\
.6\end{array}$ & $\begin{array}{r}3.7 \\
3.0 \\
10.1 \\
11.0 \\
10.2 \\
5.8 \\
2.1 \\
11.3 \\
.4 \\
3.7 \\
.4 \\
.4 . \\
.1\end{array}$ & $\begin{array}{r}2.9 \\
5.0 \\
2.8 \\
42.4 \\
59.9 \\
35.9 \\
4.5 \\
4.0 \\
Q \\
Q \\
.7 \\
9.7 \\
Q\end{array}$ & $\begin{array}{r}9.1 \\
14.1 \\
23.4 \\
23.0 \\
33.0 \\
13.5 \\
16.2 \\
23.1 \\
8.4 \\
20.5 \\
11.1 \\
49.4 \\
3.2\end{array}$ & $\begin{array}{r}2.6 \\
Q \\
27.0 \\
50.5 \\
21.9 \\
9.2 \\
1.9 \\
.7 \\
Q \\
1.3 \\
.1 \\
Q \\
Q\end{array}$ & $\begin{array}{r}1.6 \\
1.3 \\
56.5 \\
13.7 \\
3.0 \\
4.5 \\
1.0 \\
2.9 \\
.9 \\
2.9 \\
2.6 \\
4.5 \\
.5\end{array}$ & $\begin{array}{r}.9 \\
1.3 \\
1.3 \\
1.5 \\
2.6 \\
.4 \\
9.3 \\
13.9 \\
1.1 \\
2.2 \\
6.2 \\
Q \\
.5\end{array}$ & $\begin{array}{r}6.4 \\
6.1 \\
9.0 \\
18.3 \\
33.5 \\
8.8 \\
16.5 \\
9.9 \\
7.6 \\
18.6 \\
12.3 \\
82.7 \\
7.2\end{array}$ & $\begin{array}{r}10.48 \\
12.03 \\
16.05 \\
12.02 \\
10.85 \\
12.24 \\
8.37 \\
6.82 \\
23.00 \\
33.18 \\
14.29 \\
28.47 \\
33.85\end{array}$ \\
\hline 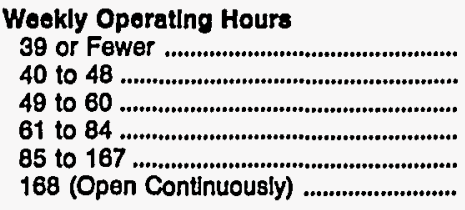 & $\begin{array}{r}33.5 \\
71.8 \\
68.6 \\
92.0 \\
106.3 \\
174.8\end{array}$ & $\begin{array}{l}19.9 \\
33.5 \\
27.6 \\
30.1 \\
34.5 \\
42.8\end{array}$ & $\begin{array}{l}1.2 \\
3.5 \\
3.4 \\
5.3 \\
6.2 \\
9.1\end{array}$ & $\begin{array}{l}1.6 \\
3.1 \\
3.9 \\
4.8 \\
4.0 \\
8.7\end{array}$ & $\begin{array}{r}1.7 \\
2.8 \\
2.4 \\
7.0 \\
6.6 \\
29.3\end{array}$ & $\begin{array}{r}3.8 \\
11.3 \\
12.5 \\
17.6 \\
20.8 \\
30.2\end{array}$ & $\begin{array}{r}.5 \\
.7 \\
1.1 \\
3.9 \\
10.8 \\
10.4\end{array}$ & $\begin{array}{l}1.0 \\
2.0 \\
1.8 \\
2.9 \\
4.7 \\
5.6\end{array}$ & $\begin{array}{l}1.1 \\
5.1 \\
6.9 \\
6.6 \\
6.0 \\
8.5\end{array}$ & $\begin{array}{r}2.8 \\
9.8 \\
9.1 \\
13.8 \\
12.7 \\
30.2\end{array}$ & $\begin{array}{r}6.43 \\
5.65 \\
6.68 \\
6.86 \\
11.77 \\
8.71\end{array}$ \\
\hline 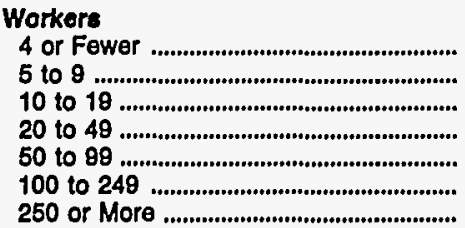 & $\begin{array}{r}46.0 \\
67.3 \\
83.8 \\
97.2 \\
94.8 \\
146.5 \\
141.0\end{array}$ & $\begin{array}{l}21.2 \\
25.8 \\
33.4 \\
40.9 \\
37.5 \\
47.4 \\
28.6\end{array}$ & $\begin{array}{l}2.2 \\
3.8 \\
4.6 \\
4.3 \\
5.0 \\
6.3 \\
9.1\end{array}$ & $\begin{array}{r}1.9 \\
2.6 \\
3.0 \\
2.8 \\
3.4 \\
6.4 \\
11.5\end{array}$ & $\begin{array}{r}4.5 \\
6.3 \\
7.3 \\
8.5 \\
3.8 \\
10.1 \\
15.7\end{array}$ & $\begin{array}{r}5.8 \\
10.4 \\
11.5 \\
16.2 \\
18.0 \\
29.7 \\
29.3\end{array}$ & $\begin{array}{r}1.5 \\
3.3 \\
4.0 \\
3.2 \\
2.8 \\
5.8 \\
10.7\end{array}$ & $\begin{array}{l}2.0 \\
2.2 \\
3.1 \\
3.4 \\
3.8 \\
4.2 \\
3.1\end{array}$ & $\begin{array}{r}2.9 \\
5.3 \\
6.6 \\
5.8 \\
5.2 \\
8.6 \\
10.1\end{array}$ & $\begin{array}{r}4.0 \\
7.7 \\
10.3 \\
12.1 \\
15.1 \\
28.0 \\
22.9\end{array}$ & $\begin{array}{r}7.15 \\
7.34 \\
7.59 \\
6.65 \\
9.85 \\
11.25 \\
10.67\end{array}$ \\
\hline
\end{tabular}

See footnote at end of table. 
Table B2. Energy End-Use Intensities for All Major Fuels, 1989 (Continued)

\begin{tabular}{|c|c|c|c|c|c|c|c|c|c|c|c|}
\hline \multirow{3}{*}{$\begin{array}{c}\text { Bullding } \\
\text { Characteristics } \\
\text { RSE Column Factor: }\end{array}$} & \multicolumn{10}{|c|}{$\begin{array}{l}\text { Energy Intensity for Major Fuels } \\
\text { (thousand Btu per sq. ft.) }\end{array}$} & \multirow{3}{*}{$\begin{array}{l}\text { RSE } \\
\text { Row } \\
\text { Factor }\end{array}$} \\
\hline & Total & $\begin{array}{c}\text { Space } \\
\text { Heating }\end{array}$ & Cooling & $\begin{array}{l}\text { Ventil- } \\
\text { ation }\end{array}$ & $\begin{array}{c}\text { Water } \\
\text { Heating }\end{array}$ & Lighting & Cooking & $\begin{array}{l}\text { Refrig- } \\
\text { eration }\end{array}$ & $\begin{array}{l}\text { Office } \\
\text { Equip- } \\
\text { ment }\end{array}$ & Other & \\
\hline & 1.0 & NF & NF & NF & NF & NF & NF & NF & NF & NF & \\
\hline \multicolumn{12}{|l|}{ Owmershlp and Occupancy } \\
\hline Nongovernment Owned ............................... & 86.8 & 27.9 & 4.9 & 4.3 & 7.7 & 15.5 & 3.9 & 3.1 & 6.4 & 13.1 & 5.15 \\
\hline 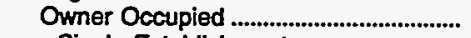 & 92.6 & 30.7 & 5.1 & 4.6 & 8.9 & 15.7 & 4.2 & 3.4 & 5.9 & 14.2 & 5.92 \\
\hline Single Establishment ............................... & 98.5 & 33.9 & 5.0 & 4.2 & 10.2 & 15.5 & 4.6 & 3.9 & 5.5 & 15.6 & 7.47 \\
\hline Multiple Establishment ............................. & 74.7 & 20.7 & 5.2 & 5.8 & 5.0 & 16.5 & 2.7 & 1.8 & 6.8 & 10.1 & 3.84 \\
\hline 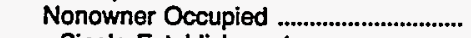 & 70.5 & 20.3 & 4.3 & 3.4 & 4.1 & 14.9 & 3.1 & 2.5 & 7.8 & 10.1 & 6.65 \\
\hline Single Establishment ................................ & 75.4 & 23.6 & 4.0 & 3.4 & 3.7 & 15.6 & 3.9 & 3.4 & 8.2 & 9.6 & 9.70 \\
\hline Multiple Establishment ........................... & 75.2 & 17.9 & 5.8 & 4.2 & 4.7 & 17.4 & 2.9 & 2.0 & 9.2 & 11.0 & 9.26 \\
\hline 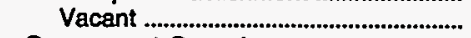 & $Q$ & 14.9 & .6 & .1 & $\mathbf{Q}$ & 2.2 & $\mathbf{Q}$ & .4 & .3 & Q & NF \\
\hline 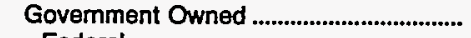 & 108.0 & 45.5 & 4.5 & 4.8 & 8.7 & 18.5 & $\mathbf{Q}$ & 2.3 & 4.8 & 13.3 & 9.84 \\
\hline 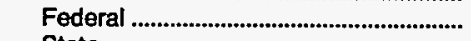 & 142.1 & 36.9 & 9.1 & 8.7 & 5.7 & 31.2 & $\mathbf{Q}$ & 2.6 & 8.5 & 14.4 & 34.01 \\
\hline State & 149.8 & 65.3 & 5.2 & 5.5 & 14.3 & 21.3 & 3.2 & 3.5 & 7.5 & 23.9 & 18.14 \\
\hline 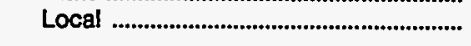 & 81.2 & 38.3 & 3.2 & 3.6 & 6.8 & 14.4 & 2.4 & 1.7 & 2.7 & 8.1 & 8.90 \\
\hline \multicolumn{12}{|l|}{ Multibullding Facility } \\
\hline Not on Multibuilding Facility ......................... & 77.5 & 28.3 & 4.1 & 3.9 & 5.9 & 13.7 & 3.1 & 3.1 & 5.7 & 9.7 & 4.11 \\
\hline $\begin{array}{l}\text { Part of Multibuilding Facility .......................... } \\
\text { On Facility with Central }\end{array}$ & 111.8 & 37.0 & 5.8 & 5.1 & 10.8 & 19.8 & 5.9 & 2.8 & 6.4 & 18.2 & 8.09 \\
\hline 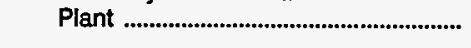 & 190.9 & 61.1 & 8.6 & 8.0 & 21.9 & 30.0 & 13.3 & 4.6 & 7.7 & 35.7 & 11.24 \\
\hline \multicolumn{12}{|l|}{$\begin{array}{l}\text { Percent Vacant at Least Three } \\
\text { Month3 }\end{array}$} \\
\hline 0 & 100.3 & 36.2 & 5.0 & 4.5 & 8.6 & 17.6 & 4.1 & 3.5 & 6.7 & 14.0 & 5.35 \\
\hline 1 to 50 & 87.3 & 24.3 & 5.6 & 5.7 & 7.6 & 17.4 & 3.2 & 2.0 & 6.4 & 15.1 & 8.84 \\
\hline 51 to 99 & 62.1 & 24.0 & $\mathbf{Q}$ & 1.9 & 3.1 & 8.0 & $\mathbf{Q}$ & 1.2 & 1.8 & 4.4 & 35.57 \\
\hline 100 & 39.5 & 16.8 & 1.5 & 1.5 & 5.0 & 5.2 & .7 & 1.5 & 1.4 & 5.9 & 17.47 \\
\hline \multicolumn{12}{|l|}{ Months in Use Out of Past 12 Months } \\
\hline 0 to 8 & 38.2 & 13.5 & 1.7 & 1.5 & 4.6 & 5.6 & 2.5 & 1.7 & 2.1 & 4.9 & 14.71 \\
\hline 9 to 11 & 72.0 & 43.0 & 1.9 & 3.0 & 5.8 & 8.4 & .8 & 1.1 & 1.1 & 6.9 & 13.70 \\
\hline 12 & 97.4 & 32.7 & 5.3 & 4.7 & 8.3 & 17.6 & 4.7 & 3.2 & 6.7 & 14.3 & 5.47 \\
\hline \multicolumn{12}{|l|}{$\begin{array}{l}\text { LOCATION } \\
\text { Census Reglon }\end{array}$} \\
\hline 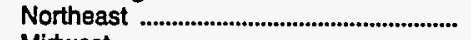 & 99.8 & 41.8 & 3.1 & 4.2 & 8.5 & 16.8 & 3.5 & 2.6 & 6.8 & 12.7 & 10.72 \\
\hline 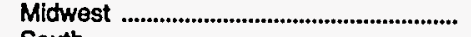 & 104.0 & 46.0 & 3.5 & 3.7 & 10.4 & 15.1 & 4.3 & 3.0 & 4.9 & 13.1 & 8.40 \\
\hline South & 74.8 & 19.2 & 6.2 & 4.2 & 5.1 & 15.5 & 2.8 & 3.0 & 5.5 & 13.2 & 7.20 \\
\hline West & 96.9 & 25.3 & 6.0 & 6.0 & 8.9 & 18.4 & $Q$ & 3.3 & 7.4 & 13.7 & 11.61 \\
\hline \multicolumn{12}{|l|}{$\begin{array}{l}\text { Census Divislon } \\
\text { Northeast }\end{array}$} \\
\hline 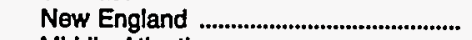 & $\mathbf{9 4 . 0}$ & 39.6 & 2.6 & 4.0 & 11.2 & 14.4 & 3.5 & 2.4 & 5.1 & 11.2 & 18.57 \\
\hline Middle Atlantic .................................................. & 101.6 & 42.4 & 3.3 & 4.2 & 7.7 & 17.5 & 3.4 & 2.7 & 7.3 & 13.1 & 12.82 \\
\hline Midwest & & & & & & & & & & & \\
\hline 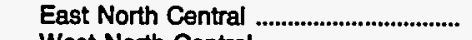 & 101.7 & 46.2 & 2.8 & 3.4 & 9.9 & 15.0 & 4.4 & 3.2 & 5.0 & 11.7 & 10.95 \\
\hline 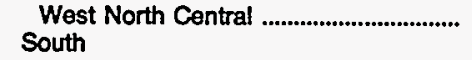 & 108.7 & 45.4 & 5.0 & 4.2 & 11.5 & 15.2 & 4.2 & 2.7 & 4.7 & 16.0 & 12.60 \\
\hline 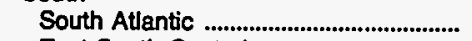 & 67.6 & 16.3 & 6.1 & 4.3 & 3.5 & 14.5 & 2.1 & 2.5 & 5.5 & 12.9 & 12.26 \\
\hline 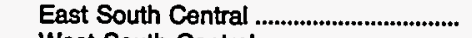 & 86.8 & 23.4 & 4.8 & 4.0 & 6.9 & 16.6 & 3.5 & 3.5 & 7.0 & 17.1 & 13.77 \\
\hline 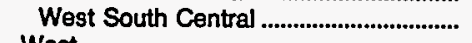 & 77.6 & 20.7 & 7.1 & 4.3 & 6.3 & 16.1 & 3.5 & 3.3 & 4.9 & 11.5 & 8.12 \\
\hline West & & & & & & & & & & & \\
\hline 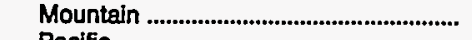 & 102.5 & 36.3 & 6.3 & 5.5 & 8.4 & 15.0 & Q & 2.7 & 5.6 & 8.7 & 18.04 \\
\hline$\ldots$ & 93.4 & 18.6 & 5.7 & 6.3 & 9.3 & 20.5 & 4.2 & 3.6 & 8.6 & 16.8 & 12.01 \\
\hline Metropolitan Status & & & & & & & & & & & \\
\hline Metropolitan ...................................................... & 94.1 & 30.7 & 5.2 & 4.8 & 8.2 & 17.2 & 4.7 & 3.1 & 6.4 & 13.8 & 5.28 \\
\hline 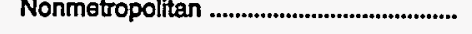 & 81.5 & 36.8 & 3.0 & 2.7 & 6.6 & 12.1 & 2.8 & 2.5 & 4.5 & 10.6 & 11.42 \\
\hline
\end{tabular}

See footnote at end of table. 
Table B2. Energy End-Use Intensities for All Major Fuels, 1989 (Continued)

\begin{tabular}{|c|c|c|c|c|c|c|c|c|c|c|c|}
\hline \multirow{3}{*}{$\begin{array}{c}\text { Bullding } \\
\text { Characteristics } \\
\text { RSE Column Factor: }\end{array}$} & \multicolumn{10}{|c|}{$\begin{array}{l}\text { Energy Intensity for Major Fuels } \\
\text { (thousand Btu per sq. ft) }\end{array}$} & \multirow{3}{*}{$\begin{array}{c}\text { RSE } \\
\text { Row } \\
\text { Factor }\end{array}$} \\
\hline & Total & $\begin{array}{c}\text { Space } \\
\text { Heating }\end{array}$ & Coollng & $\begin{array}{l}\text { Ventll- } \\
\text { ation }\end{array}$ & $\begin{array}{l}\text { Water } \\
\text { Heatling }\end{array}$ & Lighting & Cooking & $\begin{array}{l}\text { Refrig- } \\
\text { eration }\end{array}$ & $\begin{array}{l}\text { Otfice } \\
\text { Equip- } \\
\text { ment }\end{array}$ & Other & \\
\hline & 1.0 & NF & NF & NF & NF & NF & NF & NF & NF & NF & \\
\hline \multicolumn{12}{|l|}{$\begin{array}{l}\text { Climate Zone: 45-Year Average } \\
\text { Under } 2.000 \mathrm{CDD} \text { and -- }\end{array}$} \\
\hline 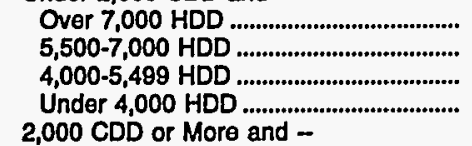 & $\begin{array}{r}121.8 \\
103.3 \\
90.5 \\
86.4\end{array}$ & $\begin{array}{l}58.9 \\
46.6 \\
29.4 \\
21.0\end{array}$ & $\begin{array}{l}2.0 \\
3.2 \\
4.3 \\
5.5\end{array}$ & $\begin{array}{l}3.2 \\
3.9 \\
4.7 \\
5.2\end{array}$ & $\begin{array}{r}13.2 \\
8.1 \\
8.3 \\
6.8\end{array}$ & $\begin{array}{l}16.6 \\
14.8 \\
18.1 \\
17.4\end{array}$ & $\begin{array}{l}3.6 \\
6.8 \\
3.4 \\
3.5\end{array}$ & $\begin{array}{l}4.5 \\
2.6 \\
2.8 \\
3.0\end{array}$ & $\begin{array}{l}6.0 \\
5.2 \\
6.6 \\
7.2\end{array}$ & $\begin{array}{l}13.9 \\
12.1 \\
12.9 \\
16.8\end{array}$ & $\begin{array}{r}13.94 \\
9.27 \\
9.34 \\
8.37\end{array}$ \\
\hline Under 4,000 HDD & 68.1 & 13.3 & 8.3 & 4.4 & 5.9 & 14.2 & 2.8 & 3.1 & 5.1 & 11.0 & 8.70 \\
\hline \multicolumn{12}{|l|}{$\begin{array}{l}1989 \text { Degreo-Days } \\
\text { Under } 2,000 \mathrm{CDD} \text { and - }\end{array}$} \\
\hline Over 7,000 HDD & 114.3 & 58.5 & 1.9 & 3.0 & 10.2 & 15.3 & 3.9 & 3.7 & 5.0 & 12.8 & 12.22 \\
\hline $\begin{array}{l}5,500-7,000 \text { HDD } \\
4,000-5,499 \text { HDD }\end{array}$ & 100.3 & 40.5 & 3.7 & 4.1 & $\begin{array}{l}9.3 \\
6.6\end{array}$ & 15.2 & $\begin{array}{l}5.8 \\
3.9\end{array}$ & 2.5 & $\begin{array}{l}5.4 \\
7.8\end{array}$ & $\begin{array}{r}13.7 \\
0.8\end{array}$ & $\begin{array}{l}8.10 \\
8.07\end{array}$ \\
\hline 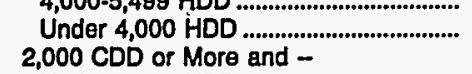 & 84.7 & $\begin{array}{l}20.0 \\
20.8\end{array}$ & 5.6 & 5.0 & 6.6 & 16.3 & 3.4 & 2.8 & 6.9 & 17.2 & 9.50 \\
\hline 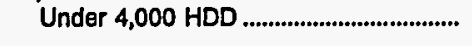 & 69.6 & 13.4 & 8.6 & 4.5 & 6.0 & 14.6 & 2.8 & 3.2 & 5.2 & 11.2 & 8.66 \\
\hline \multicolumn{12}{|l|}{$\begin{array}{l}\text { STRUCTURE } \\
\text { Floors }\end{array}$} \\
\hline 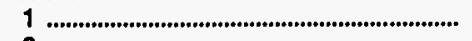 & 76.0 & 26.8 & 4.6 & 3.0 & 5.2 & 13.6 & 3.2 & 3.1 & 6.0 & 10.5 & 5.52 \\
\hline 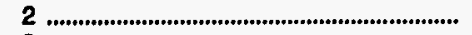 & 95.1 & 32.9 & 4.4 & 3.6 & 5.9 & 18.5 & 2.7 & 3.7 & 7.1 & 16.4 & 7.35 \\
\hline 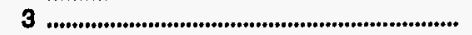 & 88.9 & 39.5 & 3.5 & 4.0 & 7.3 & 12.4 & 2.1 & 1.8 & 4.4 & 13.9 & 13.73 \\
\hline 4 to $6 \ldots$ & 107.5 & 38.7 & 5.4 & 5.1 & 13.4 & 17.3 & $\mathbf{Q}$ & 2.4 & 4.2 & 10.6 & 11.16 \\
\hline 7 or More & 123.7 & 29.2 & 7.5 & 11.4 & 16.8 & 23.7 & 7.2 & 3.0 & 7.9 & 17.0 & 11.84 \\
\hline \multicolumn{12}{|l|}{ Wall Materials } \\
\hline (n) & 93.2 & 34.8 & 4.7 & 4.0 & 9.1 & 15.1 & 4.1 & 3.0 & 5.5 & 12.8 & 4.96 \\
\hline 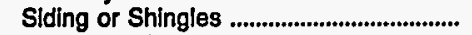 & 68.0 & 24.7 & 3.2 & 2.6 & 7.7 & 11.6 & 2.9 & 2.1 & 4.7 & 8.4 & 9.84 \\
\hline 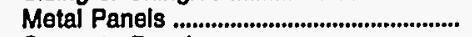 & 80.4 & 28.4 & 3.8 & 3.3 . & 3.2 & 17.0 & 1.0 & 2.6 & 7.3 & 13.8 & 20.05 \\
\hline 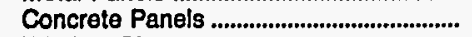 & 97.7 & 23.8 & 6.4 & $5.3^{\circ}$ & 5.0 & 20.0 & $\mathbf{Q}$ & 3.3 & 6.4 & 18.5 & 15.57 \\
\hline 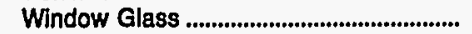 & 116.4 & 27.7 & 6.9 & 13.6 & 9.0 & 27.9 & $\mathbf{Q}$ & 3.8 & 12.6 & 10.2 & 14.95 \\
\hline 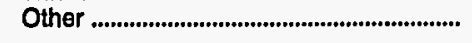 & 105.1 & 30.8 & 5.0 & 9.6 & 5.9 & 25.9 & 2.0 & 2.4 & 9.8 & 13.6 & 25.71 \\
\hline \multicolumn{12}{|l|}{ Roof Materials } \\
\hline Built-Up .......................... & 97.2 & 32.8 & 5.5 & 4.9 & 7.9 & 17.9 & 5.0 & 3.0 & 6.2 & $\begin{array}{r}14.0 \\
79\end{array}$ & $\begin{array}{r}7.03 \\
6.79\end{array}$ \\
\hline Shingles (Not Wood) ... & 72.8 & 29.9 & 3.7 & 3.2 & 7.0 & 10.9 & 3.3 & 2.9 & 4.1 & 7.9 & $\begin{array}{r}6.79 \\
1625\end{array}$ \\
\hline 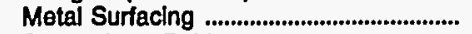 & 72.8 & 26.6 & 2.6 & 2.1 & 2.8 & 14.4 & 1.5 & 2.4 & 7.2 & 13.1 & 16.25 \\
\hline 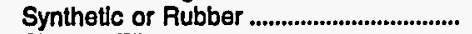 & 123.0 & 41.6 & 6.0 & 6.5 & 11.2 & 21.8 & 4.4 & 4.4 & 8.2 & 18.9 & 10.56 \\
\hline 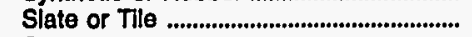 & 79.8 & 36.6 & 3.3 & 3.0 & 7.6 & 8.8 & 7.3 & 2.2 & $\mathbf{Q}$ & 7.2 & 15.64 \\
\hline 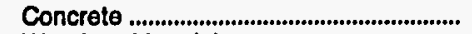 & 57.4 & 10.3 & 4.4 & 6.2 & 7.3 & 14.3 & Q & 1.7 & 5.0 & 5.5 & 13.60 \\
\hline 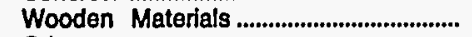 & B7.2 & 32.4 & 6.0 & 4.9 & 9.5 & 12.7 & 5.7 & 3.3 & 3.5 & 9.0 & 11.88 \\
\hline 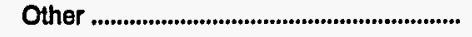 & 172.1 & 31.4 & 8.2 & 7.2 & 40.9 & 22.5 & 10.0 & 2.4 & 5.8 & $\mathbf{Q}$ & 28.73 \\
\hline \multicolumn{12}{|l|}{$\begin{array}{l}\text { Bullding Shell Conservation } \\
\text { Features (Solely or In Comblnation) }\end{array}$} \\
\hline Roof or Celling Insulation ........................... & 99.5 & 33.3 & 5.6 & 4.9 & 9.0 & 18.2 & 4.9 & 3.3 & 6.8 & 13.5 & 5.47 \\
\hline 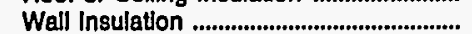 & 102.9 & 31.7 & 5.9 & 5.0 & 9.2 & 19.7 & 5.6 & 3.5 & 7.3 & 15.0 & 6.77 \\
\hline $\begin{array}{l}\text { Storm or Multiple Glazing ........ } \\
\text { Tinted, Reflectlve, or Shading }\end{array}$ & 106.2 & 35.2 & 5.4 & 5.3 & 11.9 & 18.9 & 5.2 & 3.8 & 7.5 & 12.9 & 5.24 \\
\hline 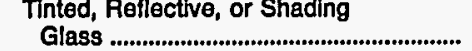 & 108.2 & 29.8 & 6.8 & 6.5 & 8.8 & 22.2 & 6.1 & 3.4 & 8.6 & 16.0 & 7.29 \\
\hline Exterior or Interior Shadings & & & & & & & & & & & \\
\hline 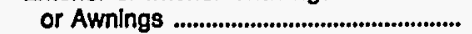 & 103.9 & 32.4 & 6.1 & 5.7 & 8.8 & 18.9 & 6.2 & 3.1 & 7.5 & 15.3 & 6.84 \\
\hline Weather Stripping or Caulking .................. & 101.8 & 34.2 & 5.6 & 5.1 & 9.0 & 18.5 & 5.2 & 3.2 & 6.9 & 13.9 & 4.76 \\
\hline 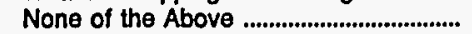 & 43.2 & 16.2 & 1.6 & 1.6 & 3.6 & 6.6 & 1.5 & 1.5 & 2.5 & 8.2 & 16.63 \\
\hline
\end{tabular}

See footnote at end of table. 
Table B2. Energy End-Use Intensities for All Major Fuels, 1989 (Continued)

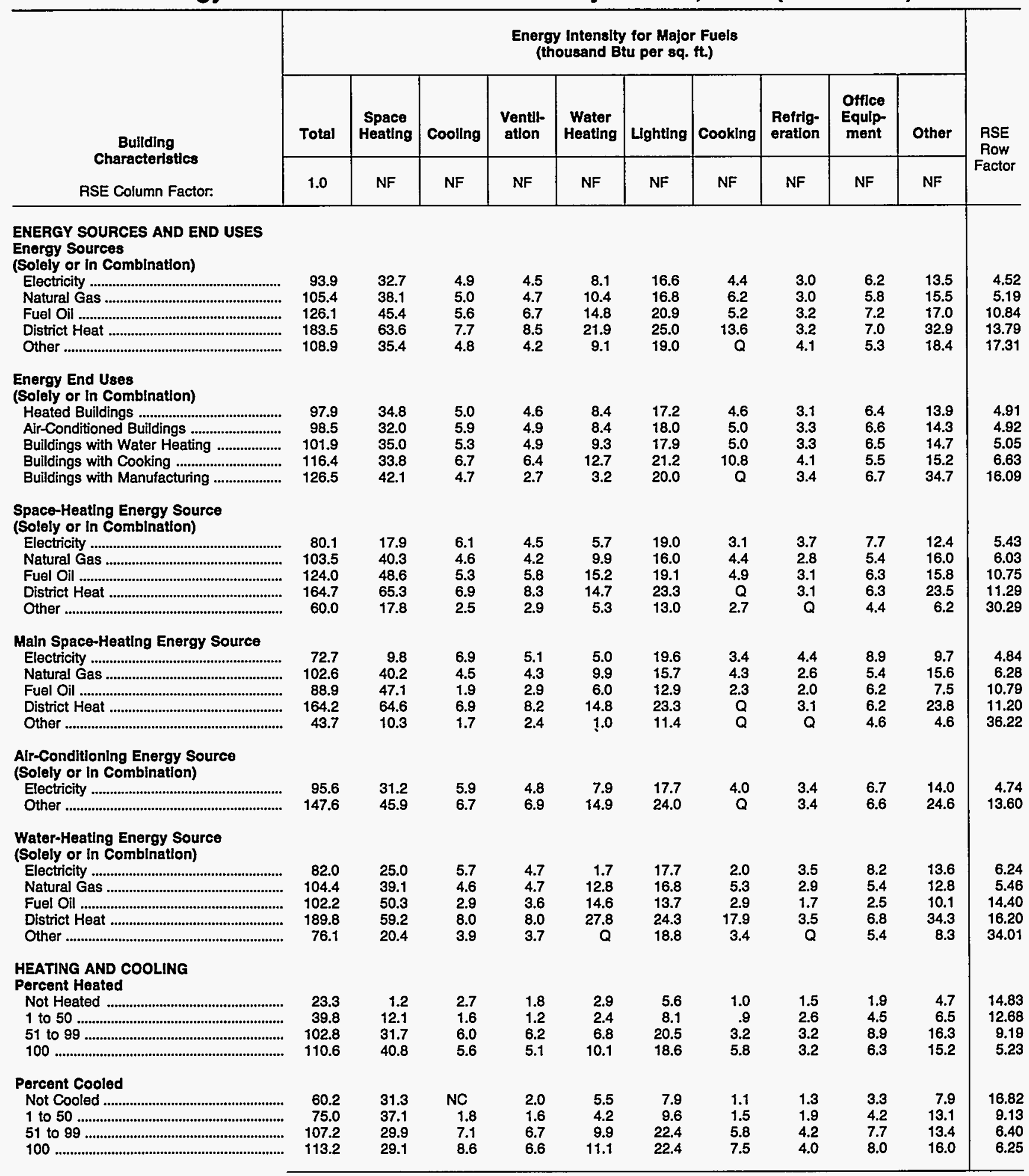

See footnotes at end of table. 
Table B2. Energy End-Use Intensities for All Major Fuels, 1989 (Continued)

\begin{tabular}{|c|c|c|c|c|c|c|c|c|c|c|c|}
\hline \multirow{3}{*}{$\begin{array}{c}\text { Bullding } \\
\text { Characteristics } \\
\text { RSE Column Factor: }\end{array}$} & \multicolumn{10}{|c|}{$\begin{array}{l}\text { Energy Intenslty for Major Fuels } \\
\text { (thousand Btu per sq. ft) }\end{array}$} & \multirow{3}{*}{$\begin{array}{c}\text { RSE } \\
\text { Row } \\
\text { Factor }\end{array}$} \\
\hline & Total & $\begin{array}{l}\text { Space } \\
\text { Heating }\end{array}$ & Coollng & $\begin{array}{l}\text { Ventil } \\
\text { ation }\end{array}$ & $\begin{array}{c}\text { Water } \\
\text { Heating }\end{array}$ & Llghting & Cooklng & $\begin{array}{l}\text { Refrlg- } \\
\text { eration }\end{array}$ & $\begin{array}{c}\text { Offlce } \\
\text { Equip- } \\
\text { ment }\end{array}$ & Other & \\
\hline & 1.0 & NF & NF & NF & NF & NF & NF & NF & NF & NF & \\
\hline \multicolumn{12}{|l|}{$\begin{array}{l}\text { Heating Equlpment } \\
\text { (Solely or in Combination) }\end{array}$} \\
\hline 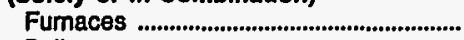 & 89.0 & 36.1 & 3.6 & 2.9 & 6.1 & 14.1 & 3.4 & 3.4 & 6.4 & 13.3 & 7.95 \\
\hline 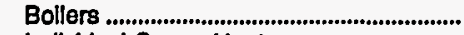 & 113.0 & 48.0 & 4.5 & 5.4 & 10.6 & 17.8 & 3.8 & 2.8 & 5.2 & 14.9 & 5.63 \\
\hline Individual Space Heaters ............................... & 90.1 & 31.4 & 4.1 & 3.6 & 7.2 & 15.9 & 4.6 & 2.4 & 5.9 & 14.9 & 7.90 \\
\hline 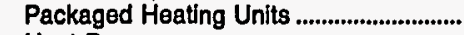 & 99.3 & 26.7 & 6.6 & 4.4 & 6.4 & 20.3 & 4.9 & 3.2 & 7.5 & 19.4 & 7.53 \\
\hline 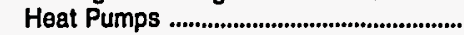 & 87.4 & 22.6 & 6.0 & 5.1 & 6.5 & 17.7 & 3.3 & 3.7 & 6.9 & 15.6 & 9.94 \\
\hline 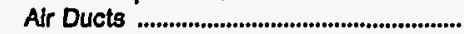 & 106.8 & 33.2 & 6.0 & 5.5 & 9.3 & 20.2 & 5.7 & 3.4 & 7.4 & 16.2 & 5.41 \\
\hline Heating or Reheating Coils .......................... & 133.7 & 38.4 & 7.3 & 8.1 & 13.0 & 25.6 & 8.1 & 3.3 & 7.9 & 22.0 & 7.57 \\
\hline 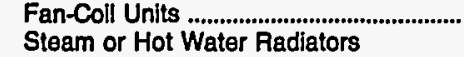 & 133.3 & 43.4 & 6.4 & 6.7 & 14.6 & 23.0 & 9.5 & 2.9 & 6.5 & 20.3 & 9.53 \\
\hline or Baseboards & $\begin{array}{l}124.7 \\
174.6\end{array}$ & $\begin{array}{l}54.2 \\
39.7\end{array}$ & $\begin{array}{l}3.9 \\
9.4\end{array}$ & $\begin{array}{r}4.9 \\
12.2\end{array}$ & $\begin{array}{l}13.4 \\
27.6\end{array}$ & $\begin{array}{l}16.2 \\
27.8\end{array}$ & $\begin{array}{l}7.9 \\
5.7\end{array}$ & $\begin{array}{l}2.2 \\
6.0\end{array}$ & $\begin{array}{l}4.5 \\
9.3\end{array}$ & $\begin{array}{l}17.6 \\
36.9\end{array}$ & $\begin{array}{r}9.10 \\
20.74\end{array}$ \\
\hline \multicolumn{12}{|l|}{$\begin{array}{l}\text { Cooling Equipment } \\
\text { (Solely or in Comblnation) }\end{array}$} \\
\hline 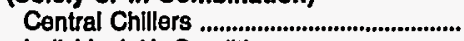 & 126.4 & 31.4 & 8.6 & 9.4 & 13.0 & 24.5 & 9.7 & 3.0 & 7.7 & 19.1 & 9.05 \\
\hline Individual Air Conditioners ............................ & 95.6 & 38.8 & 4.1 & 3.6 & 9.4 & 14.0 & 3.7 & 2.7 & 4.5 & 14.7 & 8.69 \\
\hline Packaged Cooling Units ................................... & 99.8 & 31.4 & 6.3 & 4.9 & 7.5 & 18.9 & 5.8 & 3.5 & 7.0 & 14.5 & 5.15 \\
\hline 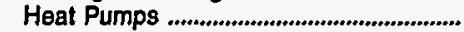 & 98.7 & 24.3 & 6.2 & 5.1 & 6.4 & 19.4 & 4.0 & 3.9 & 8.1 & 21.3 & 13.69 \\
\hline 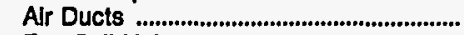 & 107.2 & 32.4 & 6.5 & 5.7 & 9.9 & 20.3 & 6.1 & 3.4 & 7.2 & 15.7 & 4.96 \\
\hline 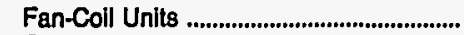 & 141.5 & 35.7 & 8.7 & 10.0 & 16.4 & 26.7 & 11.3 & 3.2 & 8.3 & 21.3 & 9.52 \\
\hline 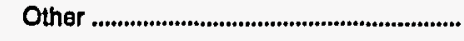 & 118.9 & 24.5 & 11.3 & 3.3 & $\mathbf{Q}$ & 19.7 & 35.7 & 1.8 & 5.7 & 8.3 & 24.32 \\
\hline \multicolumn{12}{|l|}{ Year Maln Contral Chllier Installed } \\
\hline 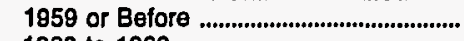 & 118.5 & 36.8 & 8.2 & 10.8 & 13.6 & 21.7 & 6.1 & 2.5 & 7.0 & 12.0 & 13.11 \\
\hline 1980 to 1969 & 141.3 & 39.8 & 10.7 & 7.7 & 7.4 & 24.2 & $\mathbf{Q}$ & 2.6 & 7.0 & 24.7 & 22.49 \\
\hline 1970 to 1979 & 116.6 & 28.0 & 7.4 & 9.2 & 15.3 & 25.2 & 6.2 & $\mathbf{3 . 0}$ & 8.1 & 14,2 & 9.02 \\
\hline 1880 to 1986 & 131.5 & 28.6 & 8.4 & 11.8 & 17.2 & 26.5 & 5.5 & 3.5 & 8.7 & 21.4 & 15.28 \\
\hline 1887 to 1889 & 111.3 & 21.9 & 7.7 & 7.8 & 11.3 & 22.1 & 11.9 & 3.6 & 6.6 & 18.4 & 19.39 \\
\hline \multicolumn{12}{|l|}{$\begin{array}{l}\text { Yoar Packaged Coollng System } \\
\text { Installed }\end{array}$} \\
\hline 1959 or Before & 99.3 & 37.2 & 6.1 & 5.3 & 7.9 & 20.7 & 2.0 & 1.8 & 5.3 & 13.1 & 12.62 \\
\hline 1980 to 1969 & 125.5 & 41.1 & 7.1 & 4.4 & 7.1 & 20.3 & $\mathbf{Q}$ & 2.6 & 7.5 & 22.5 & 16.95 \\
\hline 1970 to 1979 & 99.5 & 31.5 & 6.6 & 5.0 & 8.6 & 19.5 & 5.9 & 3.4 & 6.9 & 12.0 & 6.40 \\
\hline 1980 to 1986 & 88.4 & 24.5 & 5.8 & 4.9 & 6.4 & 18.8 & 3.6 & 4.2 & 7.8 & 12.5 & 8.17 \\
\hline 1987 to 1989 & 101.2 & 34.5 & 6.3 & 4.9 & 7.6 & 16.3 & 5.3 & 3.7 & 5.9 & 16.7 & 11.70 \\
\hline $\begin{array}{l}\text { Computer Area wlth Separate } \\
\text { Alr-Conditloning System }\end{array}$ & & & & & & & & & & & \\
\hline 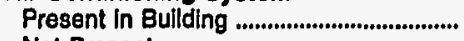 & 136.3 & 37.1 & 7.9 & 8.7 & 11.3 & 27.1 & 7.1 & 3.8 & 10.9 & 22.4 & 7.24 \\
\hline 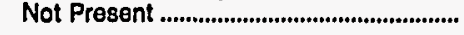 & 75.6 & 30.1 & 3.7 & 2.9 & 6.7 & 12.3 & 3.3 & 2.7 & 4.3 & 9.9 & 5.15 \\
\hline $\begin{array}{l}\text { LIGHTING AND REFRIQERATION } \\
\text { Porcent Lt When Open }\end{array}$ & & & & & & & & & & & \\
\hline 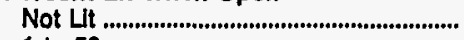 & 8.1 & 4.9 & .2 & .2 & Q & .8 & - & .3 & .5 & 1.9 & 22.17 \\
\hline 1 to 50 & 48.1 & 25.0 & 1.8 & 2.0 & 4.7 & 4.4 & 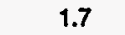 & 1.4 & 2.9 & 5.2 & 8.38 \\
\hline 61 to 89 & 85.8 & 33.4 & 4.9 & 5.0 & 7.4 & 17.3 & 3.8 & 2.5 & 6.7 & 14.9 & 7.53 \\
\hline 100 & 109.3 & 35.4 & 6.0 & 5.2 & 9.8 & 20.6 & 5.7 & 3.9 & 7.0 & 15.7 & 5.76 \\
\hline Percent Lit When Closed & & & & & & & & & & & \\
\hline 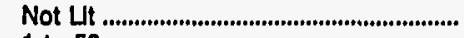 & 76.5 & 30.1 & 3.8 & 3.0 & 7.0 & 11.0 & 2.8 & 2.2 & 4.8 & 11.7 & 6.47 \\
\hline 1 to 50 & 98.9 & 33.4 & 5.1 & 5.0 & 7.1 & 19.3 & 4.8 & 3.2 & 6.9 & 14.1 & 6.04 \\
\hline 51 to 89 & 176.5 & 38.4 & 10.7 & 11.5 & 31.1 & 35.0 & 14.0 & 5.7 & 9.7 & 20.4 & 14.68 \\
\hline 100 & 87.3 & 20.1 & 10.2 & 6.6 & 5.9 & 20.0 & 5.8 & $\mathbf{Q}$ & 1.8 & 7.4 & 23.17 \\
\hline $\begin{array}{l}\text { Lightlng Equlpment } \\
\text { (Sololy or In Combination) }\end{array}$ & & & & & & & & & & & \\
\hline Incandescent Lamps ..................................... & 97.6 & 33.8 & 5.1 & 4.7 & 10.0 & 17.7 & 5.6 & 3.0 & 5.3 & 12.4 & 5.53 \\
\hline 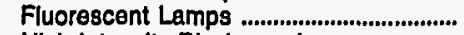 & 86.5 & 33.4 & 5.1 & 4.7 & 8.2 & 17.1 & 4.5 & 3.1 & 6.4 & 13.8 & 4.87 \\
\hline Hlgh-Intensity Discharge Lamps ............. & 108.9 & 34.4 & 5.6 & 5.2 & 7.1 & 21.7 & 6.2 & 3.5 & 6.7 & 18.4 & 7.76 \\
\hline 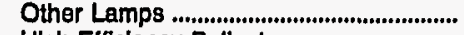 & 88.5 & 21.3 & 7.4 & 7.0 & 11.9 & 22.7 & 5.3 & $\mathbf{Q}$ & 5.2 & 13.4 & 16.93 \\
\hline Hlgh-Efficlency Ballasts ................................ & 112.7 & 35.4 & 6.1 & 6.3 & 8.4 & 20.2 & 4.5 & 4.1 & 8.8 & 17.9 & 6.61 \\
\hline
\end{tabular}

See footnotes at end of table. 
Table B2. Energy End-Use Intensities for All Major Fuels, 1989 (Continued)

\begin{tabular}{|c|c|c|c|c|c|c|c|c|c|c|c|}
\hline \multirow{3}{*}{$\begin{array}{c}\text { Bullding } \\
\text { Characteristics } \\
\text { RSE Column Factor: }\end{array}$} & \multicolumn{10}{|c|}{$\begin{array}{l}\text { Energy Intensity for Major Fuels } \\
\text { (thousand Btu per sq. ft.) }\end{array}$} & \multirow{3}{*}{$\begin{array}{l}\text { ASE } \\
\text { Pow } \\
\text { Factor }\end{array}$} \\
\hline & \multirow[t]{2}{*}{ Total } & \multirow{2}{*}{\begin{tabular}{|c|}
$\begin{array}{c}\text { Space } \\
\text { Heating }\end{array}$ \\
NF
\end{tabular}} & \multirow{2}{*}{\begin{tabular}{|c|} 
Cooling \\
NF
\end{tabular}} & \multirow{2}{*}{$\begin{array}{c}\begin{array}{c}\text { Ventll- } \\
\text { atlon }\end{array} \\
\text { NF }\end{array}$} & \multirow{2}{*}{$\begin{array}{c}\begin{array}{c}\text { Water } \\
\text { Heating }\end{array} \\
\text { NF }\end{array}$} & \multirow{2}{*}{$\begin{array}{c}\text { Lighting } \\
\text { NF }\end{array}$} & \multirow{2}{*}{\begin{tabular}{|c|} 
Cooklng \\
NF
\end{tabular}} & \multirow{2}{*}{$\begin{array}{c}\begin{array}{l}\text { Refrig- } \\
\text { eration }\end{array} \\
\text { NF }\end{array}$} & \multirow{2}{*}{$\begin{array}{c}\begin{array}{c}\text { Offlce } \\
\text { Equlp- } \\
\text { ment }\end{array} \\
\text { NF }\end{array}$} & \multirow{2}{*}{ Other } & \\
\hline & & & & & & & & & & & \\
\hline \multicolumn{12}{|l|}{$\begin{array}{l}\text { Refrigeration Equipment } \\
\text { (Solely or In Combination) } \\
\text { Commercial }\end{array}$} \\
\hline 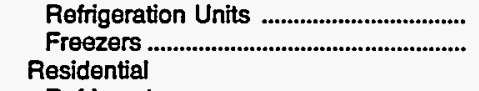 & $\begin{array}{l}120.6 \\
129.3\end{array}$ & $\begin{array}{l}33.8 \\
34.6\end{array}$ & $\begin{array}{l}7.0 \\
7.7\end{array}$ & $\begin{array}{l}6.5 \\
6.9\end{array}$ & $\begin{array}{l}12.9 \\
14.3\end{array}$ & $\begin{array}{l}22.4 \\
24.3\end{array}$ & $\begin{array}{l}10.2 \\
11.2\end{array}$ & $\begin{array}{l}5.2 \\
5.7\end{array}$ & $\begin{array}{l}6.4 \\
6.8\end{array}$ & $\begin{array}{l}16.3 \\
17.7\end{array}$ & $\begin{array}{l}5.99 \\
5.62\end{array}$ \\
\hline 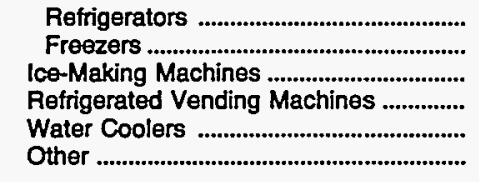 & $\begin{array}{r}99.6 \\
119.0 \\
127.5 \\
111.9 \\
103.9 \\
245.4\end{array}$ & $\begin{array}{l}35.0 \\
36.1 \\
32.3 \\
35.7 \\
35.3 \\
42.0\end{array}$ & $\begin{array}{r}5.0 \\
6.8 \\
8.2 \\
6.0 \\
5.5 \\
15.6\end{array}$ & $\begin{array}{l}4.9 \\
5.1 \\
7.6 \\
5.7 \\
5.2 \\
9.8\end{array}$ & $\begin{array}{r}8.6 \\
13.9 \\
14.5 \\
10.0 \\
8.5 \\
31.1\end{array}$ & $\begin{array}{l}17.9 \\
20.0 \\
24.0 \\
21.0 \\
18.9 \\
39.0\end{array}$ & $\begin{array}{r}4.3 \\
10.4 \\
10.3 \\
5.8 \\
4.5 \\
12.3\end{array}$ & $\begin{array}{l}2.6 \\
3.4 \\
4.7 \\
3.6 \\
2.9 \\
7.3\end{array}$ & $\begin{array}{r}6.6 \\
5.2 \\
7.0 \\
7.3 \\
6.7 \\
15.5\end{array}$ & $\begin{array}{l}14.7 \\
18.2 \\
18.9 \\
16.7 \\
16.3 \\
72.8\end{array}$ & $\begin{array}{r}6.13 \\
10.67 \\
5.94 \\
5.22 \\
5.65 \\
22.72\end{array}$ \\
\hline \multicolumn{12}{|l|}{$\begin{array}{l}\text { ENERGY MANAGEMENT } \\
\text { Occupant Control }\end{array}$} \\
\hline 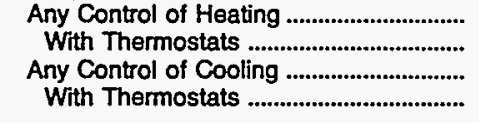 & $\begin{array}{l}86.2 \\
87.1 \\
88.2 \\
87.9\end{array}$ & $\begin{array}{l}29.8 \\
29.8 \\
29.8 \\
28.8\end{array}$ & $\begin{array}{l}4.6 \\
4.7 \\
5.2 \\
5.3\end{array}$ & $\begin{array}{l}3.6 \\
3.6 \\
3.8 \\
3.8\end{array}$ & $\begin{array}{l}9.3 \\
9.6 \\
9.4 \\
9.4\end{array}$ & $\begin{array}{l}14.5 \\
14.6 \\
15.0 \\
15.1\end{array}$ & $\begin{array}{l}4.1 \\
4.0 \\
4.3 \\
4.4\end{array}$ & $\begin{array}{l}2.5 \\
2.4 \\
2.6 \\
2.6\end{array}$ & $\begin{array}{l}5.4 \\
5.5 \\
5.6 \\
5.6\end{array}$ & $\begin{array}{l}12.4 \\
13.0 \\
12.5 \\
13.1\end{array}$ & $\begin{array}{l}7.08 \\
7.65 \\
6.94 \\
7.48\end{array}$ \\
\hline 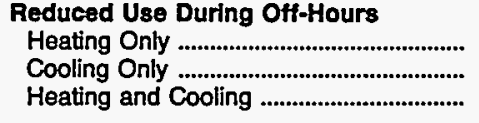 & $\begin{array}{r}90.8 \\
104.3 \\
86.5\end{array}$ & $\begin{array}{l}46.6 \\
29.0 \\
30.3\end{array}$ & $\begin{array}{l}1.3 \\
6.4 \\
4.9\end{array}$ & $\begin{array}{l}2.9 \\
4.3 \\
4.6\end{array}$ & $\begin{array}{l}8.3 \\
6.8 \\
5.9\end{array}$ & $\begin{array}{l}11.9 \\
16.8 \\
15.9\end{array}$ & $\begin{array}{l}2.2 \\
5.1 \\
4.2\end{array}$ & $\begin{array}{l}2.1 \\
2.7 \\
2.5\end{array}$ & $\begin{array}{l}4.6 \\
7.4 \\
6.0\end{array}$ & $\begin{array}{l}10.9 \\
25.8 \\
12.1\end{array}$ & $\begin{array}{r}15.32 \\
17.11 \\
6.66\end{array}$ \\
\hline \multicolumn{12}{|l|}{$\begin{array}{l}\text { Computerized Energy Management } \\
\text { and Control System }\end{array}$} \\
\hline 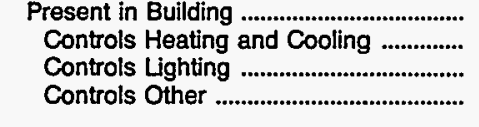 & $\begin{array}{l}119.5 \\
120.9 \\
111.9 \\
145.1\end{array}$ & $\begin{array}{l}32.0 \\
32.8 \\
24.8 \\
29.0\end{array}$ & $\begin{array}{l}7.6 \\
7.7 \\
7.8 \\
9.9\end{array}$ & $\begin{array}{l}8.0 \\
8.2 \\
5.7 \\
8.2\end{array}$ & $\begin{array}{r}11.4 \\
11.6 \\
5.2 \\
21.5\end{array}$ & $\begin{array}{l}24.8 \\
24.9 \\
22.9 \\
27.0\end{array}$ & $\begin{array}{c}7.5 \\
7.6 \\
Q \\
6.0\end{array}$ & $\begin{array}{l}3.9 \\
3.9 \\
4.1 \\
4.4\end{array}$ & $\begin{array}{l}6.9 \\
6.9 \\
6.7 \\
7.0\end{array}$ & $\begin{array}{l}17.3 \\
17.3 \\
18.2 \\
32.0\end{array}$ & $\begin{array}{r}8.29 \\
8.63 \\
13.87 \\
21.81\end{array}$ \\
\hline $\begin{array}{l}\text { Other Energy Management } \\
\text { Regular HVAC Maintenance .................... } \\
\text { Participated in Utility }\end{array}$ & 111.0 & 37.1 & 5.9 & 5.6 & 9.6 & 20.2 & 5.1 & 3.6 & 7.1 & 16.7 & 5.04 \\
\hline Conservation Program ............................. & 111.4 & 38.0 & 6.1 & 7.0 & 10.7 & 19.7 & 4.9 & 3.7 & 6.5 & 14.8 & 7.95 \\
\hline
\end{tabular}

- = Value rounds to zero in the units displayed.

$N C=$ No cases in responding sample.

$\mathrm{NF}=$ No applicable RSE row/column factor.

$Q=$ Data withheld because the Relative Standard Error (RSE) was greater than 50 percent, or data were reported for fewer than 20 buildings.

Notes: - To obtain the RSE percentage for any table cell, multiply the corresponding RSE column and RSE row factors. - See Glossary for explanation of abbreviations and definitions of terms used in this report. - Because of rounding, data may not sum to totals.

Source: Energy Information Administration, Office of Energy Markets and End Use, Forms EIA-871A through F of the 1989 Commercial Buildings Energy Consumption Survey. 


\begin{tabular}{|c|c|c|c|c|c|c|c|c|c|c|}
\hline \multirow[b]{2}{*}{$\begin{array}{l}\text { Bullding } \\
\text { Characteristics }\end{array}$} & \multicolumn{10}{|c|}{ Percent of Major Fuel Consumption } \\
\hline & Total & $\begin{array}{l}\text { Space } \\
\text { Heating }\end{array}$ & Cooling & $\begin{array}{l}\text { Ventl- } \\
\text { ation }\end{array}$ & $\begin{array}{c}\text { Water } \\
\text { Heating }\end{array}$ & | Lighting & Cooking & $\begin{array}{l}\text { Refrig- } \\
\text { eration }\end{array}$ & $\begin{array}{c}\text { Office } \\
\text { Equip- } \\
\text { ment }\end{array}$ & Other \\
\hline 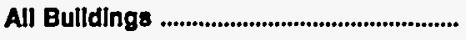 & 100 & 35 & 5 & 5 & 9 & 18 & 5 & 3 & 7 & 14 \\
\hline 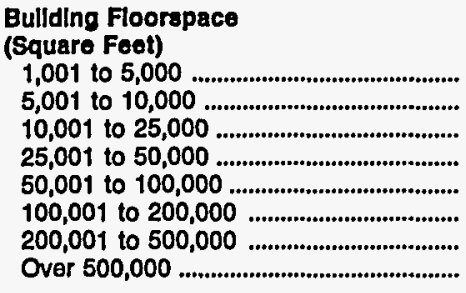 & $\begin{array}{l}100 \\
100 \\
100 \\
100 \\
100 \\
100 \\
100 \\
100\end{array}$ & $\begin{array}{l}37 \\
40 \\
41 \\
42 \\
35 \\
34 \\
29 \\
19\end{array}$ & $\begin{array}{l}6 \\
6 \\
5 \\
5 \\
4 \\
5 \\
5 \\
7\end{array}$ & $\begin{array}{l}4 \\
4 \\
3 \\
3 \\
5 \\
6 \\
7 \\
7\end{array}$ & $\begin{array}{r}11 \\
9 \\
7 \\
6 \\
8 \\
8 \\
8 \\
12\end{array}$ & $\begin{array}{l}14 \\
15 \\
16 \\
17 \\
19 \\
20 \\
22 \\
19\end{array}$ & $\begin{array}{r}7 \\
3 \\
3 \\
3 \\
3 \\
5 \\
3 \\
12\end{array}$ & $\begin{array}{l}5 \\
3 \\
4 \\
4 \\
4 \\
2 \\
2 \\
2\end{array}$ & $\begin{array}{l}8 \\
7 \\
9 \\
6 \\
5 \\
6 \\
6 \\
6\end{array}$ & $\begin{array}{r}8 \\
13 \\
12 \\
15 \\
17 \\
14 \\
18 \\
17\end{array}$ \\
\hline 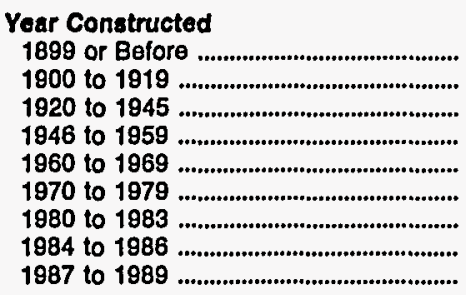 & $\begin{array}{l}100 \\
100 \\
100 \\
100 \\
100 \\
100 \\
100 \\
100 \\
100\end{array}$ & $\begin{array}{l}68 \\
46 \\
45 \\
40 \\
37 \\
30 \\
21 \\
22 \\
27\end{array}$ & $\begin{array}{l}1 \\
2 \\
4 \\
5 \\
5 \\
6 \\
7 \\
6 \\
5\end{array}$ & $\begin{array}{l}2 \\
3 \\
5 \\
4 \\
4 \\
5 \\
5 \\
8 \\
4\end{array}$ & $\begin{array}{r}9 \\
7 \\
10 \\
9 \\
9 \\
9 \\
6 \\
8 \\
7\end{array}$ & $\begin{array}{r}7 \\
12 \\
12 \\
16 \\
17 \\
21 \\
23 \\
22 \\
19\end{array}$ & $\begin{array}{l}3 \\
4 \\
4 \\
Q \\
3 \\
5 \\
4 \\
5 \\
5\end{array}$ & $\begin{array}{l}2 \\
2 \\
2 \\
2 \\
3 \\
4 \\
4 \\
5 \\
7\end{array}$ & $\begin{array}{r}2 \\
4 \\
4 \\
5 \\
6 \\
7 \\
11 \\
11 \\
10\end{array}$ & $\begin{array}{r}5 \\
21 \\
14 \\
12 \\
16 \\
13 \\
19 \\
13 \\
15\end{array}$ \\
\hline $\begin{array}{l}\text { BUILDING USE } \\
\text { Princlpal Bullding Activity }\end{array}$ & & & & & & & & & & \\
\hline $\begin{array}{l}\text { Assembly } \\
\text { Education } \\
\text { Food Sales } \\
\text { Food Service } \\
\text { Health Care } \\
\text { Lodging } \\
\text { Mercantile and Service } \\
\text { Offlce } \\
\text { Parking Garage } \\
\text { Public Order and Safety } \\
\text { Warehouse } \\
\text { Other } \\
\text { Vacant }\end{array}$ & $\begin{array}{l}100 \\
100 \\
100 \\
100 \\
100 \\
100 \\
100 \\
100 \\
100 \\
100 \\
100 \\
100 \\
100\end{array}$ & $\begin{array}{l}52 \\
54 \\
15 \\
19 \\
18 \\
32 \\
34 \\
30 \\
47 \\
53 \\
41 \\
25 \\
41\end{array}$ & $\begin{array}{r}6 \\
4 \\
11 \\
8 \\
7 \\
4 \\
5 \\
7 \\
1 \\
2 \\
1 \\
4 \\
3\end{array}$ & $\begin{array}{r}6 \\
3 \\
6 \\
5 \\
5 \\
5 \\
2 \\
11 \\
Q \\
3 \\
1 \\
-1 \\
1\end{array}$ & $\begin{array}{r}5 \\
6 \\
2 \\
19 \\
27 \\
29 \\
5 \\
4 \\
Q \\
6 \\
1 \\
4 \\
6\end{array}$ & $\begin{array}{l}14 \\
16 \\
13 \\
11 \\
15 \\
11 \\
19 \\
22 \\
20 \\
16 \\
19 \\
22 \\
14\end{array}$ & $\begin{array}{r}4 \\
Q \\
15 \\
23 \\
10 \\
8 \\
2 \\
1 \\
Q \\
1 \\
+\quad \\
2 \\
Q\end{array}$ & $\begin{array}{r}3 \\
1 \\
32 \\
6 \\
1 \\
4 \\
1 \\
3 \\
2 \\
2 \\
5 \\
2 \\
2\end{array}$ & $\begin{array}{r}1 \\
1 \\
1 \\
1 \\
1 \\
1 \\
11 \\
13 \\
3 \\
2 \\
11 \\
4 \\
2\end{array}$ & $\begin{array}{r}10 \\
7 \\
5 \\
8 \\
15 \\
7 \\
20 \\
10 \\
18 \\
15 \\
21 \\
37 \\
30\end{array}$ \\
\hline 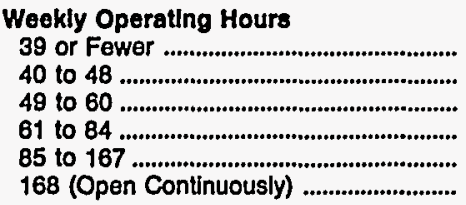 & $\begin{array}{l}100 \\
100 \\
100 \\
100 \\
100 \\
100\end{array}$ & $\begin{array}{l}60 \\
47 \\
40 \\
33 \\
32 \\
24\end{array}$ & $\begin{array}{l}3 \\
5 \\
5 \\
6 \\
6 \\
5\end{array}$ & $\begin{array}{l}5 \\
4 \\
6 \\
5 \\
4 \\
5\end{array}$ & $\begin{array}{r}5 \\
4 \\
3 \\
8 \\
6 \\
17\end{array}$ & $\begin{array}{l}11 \\
16 \\
18 \\
19 \\
20 \\
17\end{array}$ & $\begin{array}{r}2 \\
1 \\
2 \\
4 \\
10 \\
6\end{array}$ & $\begin{array}{l}3 \\
3 \\
3 \\
3 \\
4 \\
3\end{array}$ & $\begin{array}{r}3 \\
7 \\
10 \\
7 \\
6 \\
5\end{array}$ & $\begin{array}{r}8 \\
14 \\
13 \\
15 \\
12 \\
17\end{array}$ \\
\hline 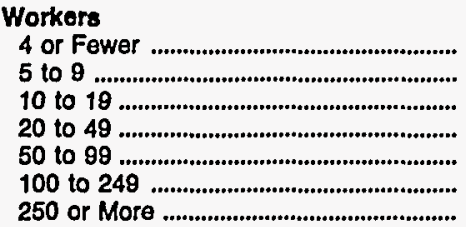 & $\begin{array}{l}100 \\
100 \\
100 \\
100 \\
100 \\
100 \\
100\end{array}$ & $\begin{array}{l}46 \\
38 \\
40 \\
42 \\
40 \\
32 \\
20\end{array}$ & $\begin{array}{l}5 \\
6 \\
6 \\
4 \\
5 \\
4 \\
6\end{array}$ & $\begin{array}{l}4 \\
4 \\
4 \\
3 \\
4 \\
4 \\
8\end{array}$ & $\begin{array}{r}10 \\
9 \\
9 \\
9 \\
4 \\
7 \\
11\end{array}$ & $\begin{array}{l}13 \\
16 \\
14 \\
17 \\
19 \\
20 \\
21\end{array}$ & $\begin{array}{l}3 \\
5 \\
5 \\
3 \\
3 \\
4 \\
8\end{array}$ & $\begin{array}{l}4 \\
3 \\
4 \\
4 \\
4 \\
3 \\
2\end{array}$ & $\begin{array}{l}6 \\
8 \\
8 \\
6 \\
6 \\
6 \\
7\end{array}$ & $\begin{array}{r}9 \\
11 \\
12 \\
12 \\
16 \\
19 \\
16\end{array}$ \\
\hline
\end{tabular}

See footnotes at end of table. 
Table B3. End-Use Consumption Percentages for All Major Fuels, 1989 (Continued)

\begin{tabular}{|c|c|c|c|c|c|c|c|c|c|c|}
\hline \multirow[b]{2}{*}{$\begin{array}{c}\text { Bullding } \\
\text { Characteristics }\end{array}$} & \multicolumn{10}{|c|}{ Percent of Major Fuel Consumption } \\
\hline & Total & $\begin{array}{l}\text { Space } \\
\text { Heating }\end{array}$ & Cooling & $\begin{array}{l}\text { Ventll- } \\
\text { ation }\end{array}$ & $\begin{array}{c}\text { Water } \\
\text { Heating }\end{array}$ & Lighting & Cooking & $\begin{array}{l}\text { Retrig- } \\
\text { eration }\end{array}$ & $\begin{array}{l}\text { Oftlce } \\
\text { Equip- } \\
\text { ment }\end{array}$ & Other \\
\hline \multicolumn{11}{|l|}{ Ownership and Occupancy } \\
\hline Nongovemment Owned ........................... & 100 & 32 & 6 & 5 & 9 & 18 & 4 & 4 & 7 & 15 \\
\hline 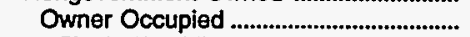 & 100 & 33 & 5 & 5 & 10 & 17 & 5 & 4 & 6 & 15 \\
\hline Single Establishment ............................. & 100 & 34 & 5 & 4 & 10 & 16 & 5 & 4 & 6 & 16 \\
\hline Multiple Establishment ........................... & 100 & 28 & 7 & 8 & 7 & 22 & 4 & 2 & 9 & 13 \\
\hline 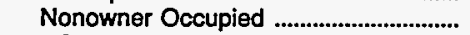 & 100 & 29 & 6 & 5 & 6 & 21 & 4 & 4 & 11 & 14 \\
\hline 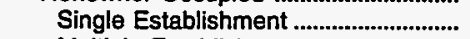 & 100 & 31 & 5 & 4 & 5 & 21 & 5 & 5 & 11 & 13 \\
\hline Multiple Establishment .......................... & 100 & 24 & 8 & 6 & 6 & 23 & 4 & 3 & 12 & 15 \\
\hline Vacant & 100 & 48 & 2 & $\cdot$ & 12 & 7 & $\mathbf{Q}$ & 1 & 1 & 28 \\
\hline 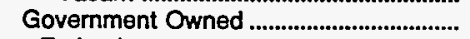 & 100 & 42 & 4 & 4 & 8 & 17 & 5 & 2 & 4 & 12 \\
\hline 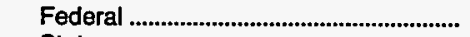 & 100 & 26 & 6 & 6 & 4 & 22 & 18 & 2 & 6 & 10 \\
\hline State & 100 & 44 & 3 & 4 & 10 & 14 & 2 & 2 & 5 & 16 \\
\hline 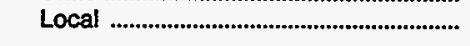 & 100 & 47 & 4 & 4 & 8 & 18 & 3 & 2 & 3 & 10 \\
\hline \multicolumn{11}{|l|}{ Multlbullding Facllity } \\
\hline Not on Multibuilding Facility ...................... & 100 & 37 & 5 & 5 & 8 & 18 & 4 & 4 & 7 & 13 \\
\hline $\begin{array}{l}\text { Part of Multibuilding Facility ........................ } \\
\text { On Facility with Central }\end{array}$ & 100 & 33 & 5 & 5 & 10 & 18 & 5 & 3 & 6 & 16 \\
\hline 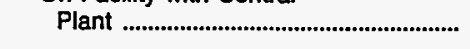 & 100 & 32 & 5 & 4 & 11 & 16 & 7 & 2 & 4 & 19 \\
\hline \multicolumn{11}{|l|}{$\begin{array}{l}\text { Percent Vacant at Least Three } \\
\text { Months }\end{array}$} \\
\hline 0 & 100 & 36 & 5 & 4 & 9 & 18 & 4 & 4 & 7 & 14 \\
\hline 1 to 50 & 100 & 28 & 6 & 7 & 9 & 20 & 4 & 2 & 7 & 17 \\
\hline 51 to 99 & 100 & 39 & 5 & 3 & 5 & 13 & 23 & $\overline{2}$ & 3 & 7 \\
\hline 100 & 100 & 43 & 4 & 4 & 13 & 13 & 2 & 4 & 4 & 15 \\
\hline \multicolumn{11}{|l|}{ Months In Use Out of Past 12 Months } \\
\hline 0 to 8 & 100 & 35 & 4 & 4 & 12 & 15 & 7 & 5 & 6 & 13 \\
\hline 9 to 11 & 100 & 60 & 3 & 4 & 8 & 12 & 1 & 2 & 1 & 10 \\
\hline 12 & 100 & 34 & 5 & 5 & 9 & 18 & 5 & 3 & 7 & 15 \\
\hline \multicolumn{11}{|l|}{$\begin{array}{l}\text { LOCATION } \\
\text { Census Reglon }\end{array}$} \\
\hline 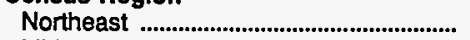 & 100 & 42 & 3 & 4 & 9 & 17 & 3 & 3 & 7 & 13 \\
\hline Midwest ............................................................... & 100 & 44 & 3 & 4 & 10 & 14 & 4 & 3 & 5 & 13 \\
\hline South & 100 & 26 & 8 & 6 & 7 & 21 & 4 & 4 & 7 & 18 \\
\hline 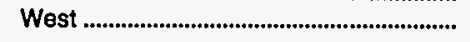 & 100 & 26 & 6 & 6 & 9 & 19 & 8 & 3 & 8 & 14 \\
\hline \multirow{2}{*}{\multicolumn{11}{|c|}{$\begin{array}{l}\text { Census Divislon } \\
\text { Northeast }\end{array}$}} \\
\hline & & & & & & & & & & \\
\hline 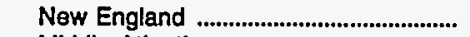 & 100 & 42 & 3 & 4 & 12 & 15 & 4 & 3 & 5 & 12 \\
\hline 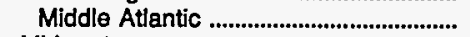 & 100 & 42 & 3 & 4 & 8 & 17 & 3 & 3 & 7 & 13 \\
\hline \multicolumn{11}{|l|}{ Midwest } \\
\hline 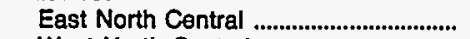 & 100 & 45 & 3 & 3 & 10 & 15 & 4 & 3 & 5 & 11 \\
\hline 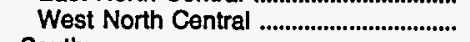 & 100 & 42 & 5 & 4 & 11 & 14 & 4 & 2 & 4 & 15 \\
\hline \multicolumn{11}{|l|}{ South } \\
\hline 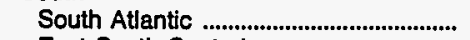 & 100 & 24 & 9 & 6 & 5 & 22 & 3 & 4 & 8 & 19 \\
\hline 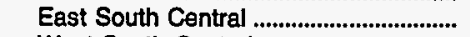 & 100 & 27 & 5 & 5 & 8 & 19 & 4 & 4 & 8 & 20 \\
\hline West South Central .................................. & 100 & 27 & 9 & 6 & 8 & 21 & 4 & 4 & 6 & 15 \\
\hline \multicolumn{11}{|l|}{ West } \\
\hline 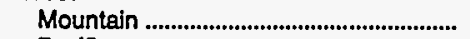 & 100 & 35 & 6 & 5 & 8 & 15 & 14 & 3 & 5 & 8 \\
\hline 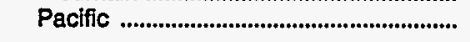 & 100 & 20 & 6 & 7 & 10 & 22 & 4 & 4 & 9 & 18 \\
\hline \multicolumn{11}{|l|}{ Metropolltan Status } \\
\hline 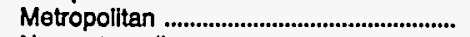 & 100 & 33 & 6 & 5 & 9 & 18 & 5 & 3 & 7 & 15 \\
\hline 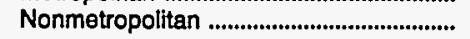 & 100 & 45 & 4 & 3 & 8 & 15 & 3 & 3 & 5 & 13 \\
\hline
\end{tabular}

See footnotes at end of table. 
Table B3. End-Use Consumption Percentages for All

Major Fuels, 1989 (Continued)

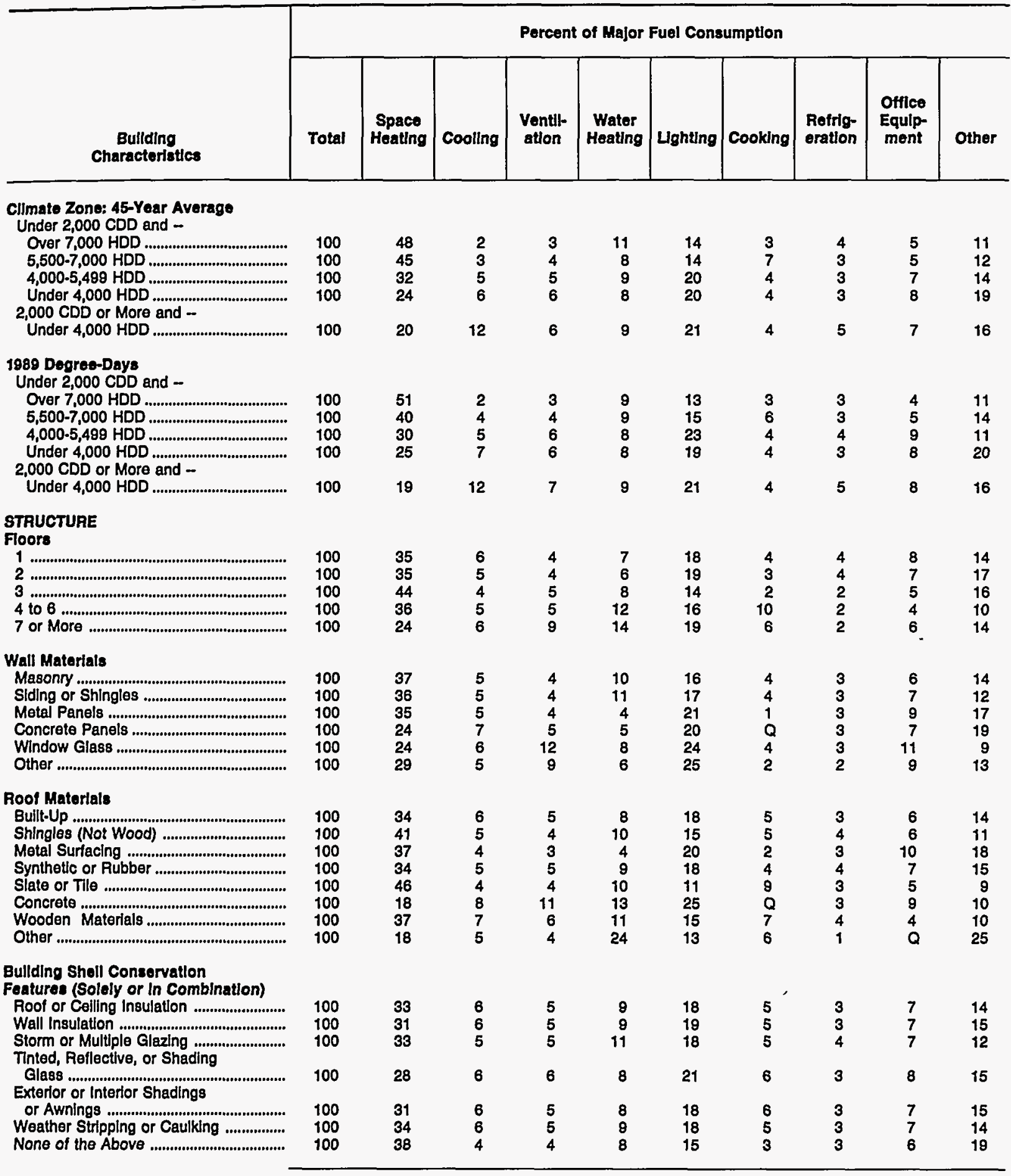

See footnotes at end of table. 
Table B3. End-Use Consumption Percentages for All Major Fuels, 1989 (Continued)

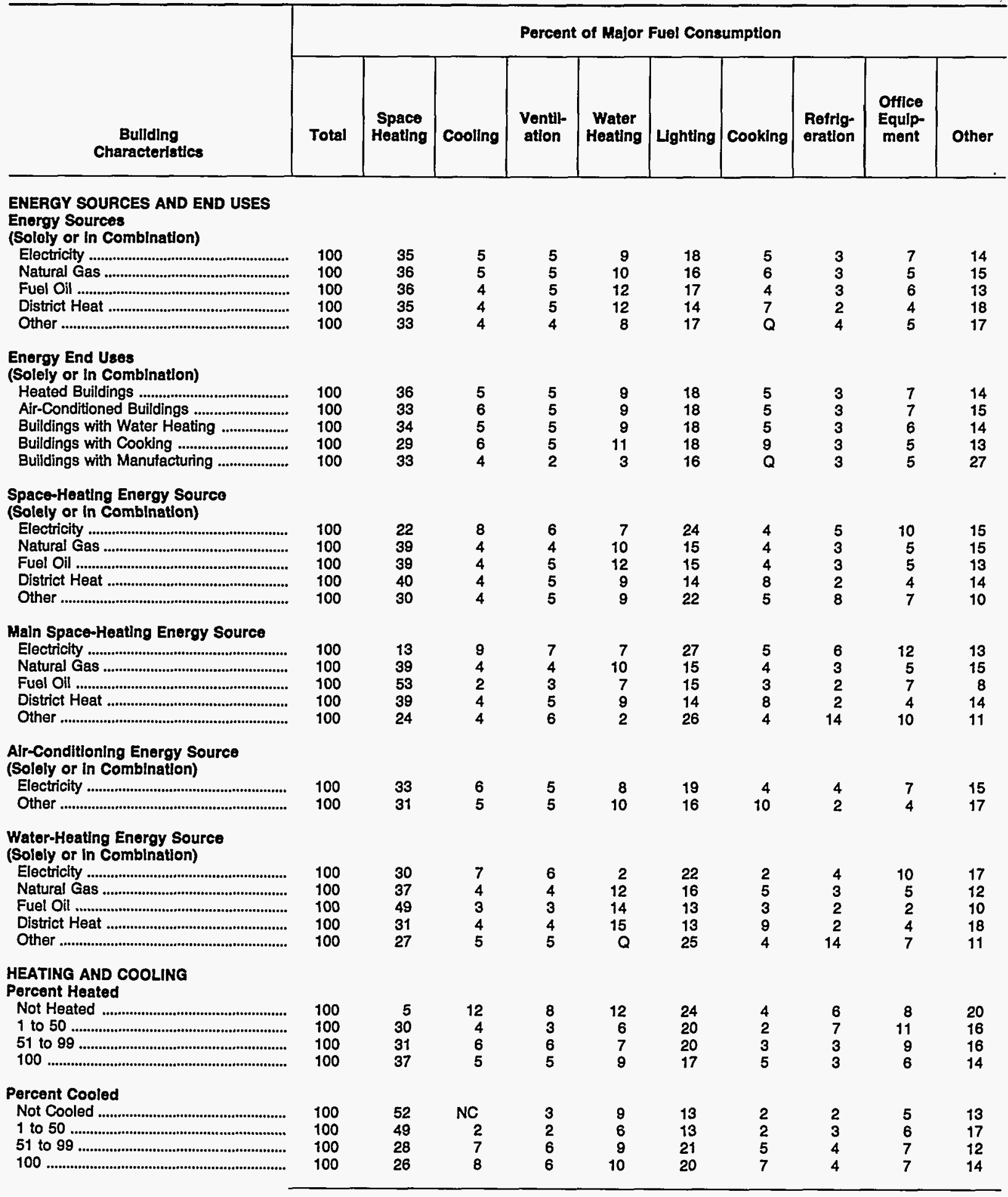

See footnotes at end of table. 
Table B3. End-Use Consumption Percentages for All Major Fuels, 1989 (Continued)

\begin{tabular}{|c|c|c|c|c|c|c|c|c|c|c|}
\hline \multirow[b]{2}{*}{$\begin{array}{c}\text { Bullding } \\
\text { Characteristlcs }\end{array}$} & \multicolumn{10}{|c|}{ Percent of Major Fuel Consumption } \\
\hline & Total & $\begin{array}{l}\text { Space } \\
\text { Heatlng }\end{array}$ & Coollng & $\begin{array}{l}\text { Yentll- } \\
\text { atlon }\end{array}$ & $\begin{array}{l}\text { Water } \\
\text { Heating }\end{array}$ & Lighting & Cooking & $\begin{array}{l}\text { Refrig- } \\
\text { eration }\end{array}$ & $\begin{array}{l}\text { Oftlce } \\
\text { Equlp- } \\
\text { ment }\end{array}$ & Other \\
\hline
\end{tabular}

Heating Equipment

(Solely or in Combination)

Fumaces

100

100

Packaged Heating Units ............................... 100

Heat Pumps ................................................... 100

Alr Ducts

Heating or Reheating Colls ..................... $\quad 100$

Fan-Coll Units .............................................. 100

Steam or Hot Water Radiators

or Baseboards ..... $\quad 100$

Other .............................................................. 100

$\begin{array}{lllllllll}41 & 4 & 3 & 7 & 16 & 4 & 4 & 7 & 15 \\ 42 & 4 & 5 & 9 & 16 & 3 & 2 & 5 & 13 \\ 35 & 5 & 4 & 8 & 18 & 5 & 3 & 7 & 17 \\ 27 & 7 & 4 & 6 & 20 & 5 & 3 & 8 & 20 \\ 26 & 7 & 6 & 7 & 20 & 4 & 4 & 8 & 18 \\ 31 & 6 & 5 & 9 & 19 & 5 & 3 & 7 & 15 \\ 29 & 5 & 6 & 10 & 19 & 6 & 2 & 6 & 16 \\ 33 & 5 & 5 & 11 & 17 & 7 & 2 & 5 & 15 \\ 43 & 3 & 4 & 11 & 13 & 6 & 2 & 4 & 14 \\ 23 & 5 & 7 & 16 & 16 & 3 & 3 & 5 & 21\end{array}$

Cooling Equipment

(Solely or in Combination)

Central Chillers ............................................ 100

Individual Air Conditioners ......................... 100

Packaged Cooling Units ............................ 100

Heat Pumps .................................................. 100

Alr Ducts .......................................................... 100

Fan-Coll Units ........................................... 100

Other 100

Year Main Central Chiller Installed

1959 or Before

100

1970 to 1979 ................................................. 100

1980 to $1986 \ldots$

1987 to 1989

100

Year Packaged Cooling System

Invtalled

1959 or Before

1960 to 1889

1970 to 1879

1980 to 1988

1987 to 1989

100

100

100

100

100

Computer Area with Separate

Alr-Conditloning System

Present in Building 100

Not Present ........................... 100

LIGHTINQ AND REFRIGERATION

Percent LIt Whon Open

Not Lt ............................................................. 100

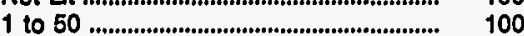

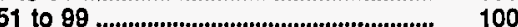

100

Percent Llt When Closed

Not LIt

1 to 50

100

100

100

100

Uahting Equlpment

(solely or In Combination)

incandescent Lamps .

100

.......... 100

Other Lamps

High-Efflciency Ballasts

See footnotes at end of table. 


\begin{tabular}{|c|c|c|c|c|c|c|c|c|c|c|}
\hline \multirow[b]{2}{*}{$\begin{array}{c}\text { Bullding } \\
\text { Characteristics }\end{array}$} & \multicolumn{10}{|c|}{ Percent of Major Fuel Consumption } \\
\hline & Total & $\begin{array}{l}\text { Space } \\
\text { Heating }\end{array}$ & Cooling & $\begin{array}{l}\text { Ventl- } \\
\text { ation }\end{array}$ & $\begin{array}{l}\text { Water } \\
\text { Heating }\end{array}$ & Llghting & Cooking & $\begin{array}{l}\text { Refrig- } \\
\text { eration }\end{array}$ & $\begin{array}{l}\text { Offlce } \\
\text { Equip- } \\
\text { ment }\end{array}$ & Other \\
\hline \multicolumn{11}{|l|}{$\begin{array}{l}\text { Retrigeration Equlpment } \\
\text { (Solely or in Comblnation) } \\
\text { Commercial }\end{array}$} \\
\hline 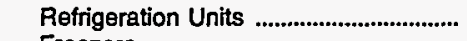 & 100 & 28 & 6 & 5 & 11 & 19 & 8 & 4 & 5 & 13 \\
\hline 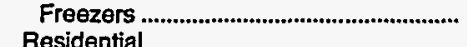 & 100 & 27 & 6 & 5 & 11 & 19 & 9 & 4 & 5 & 14 \\
\hline \multicolumn{11}{|l|}{ Residential } \\
\hline Refrigerators ................................................. & 100 & 35 & 5 & 5 & 9 & 18 & 4 & 3 & 7 & 15 \\
\hline Freezers ……................................................ & 100 & 30 & 6 & 4 & 12 & 17 & 9 & 3 & 4 & 15 \\
\hline 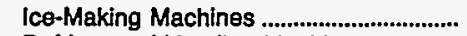 & 100 & 25 & 6 & 6 & 11 & 19 & 8 & 4 & 5 & 15 \\
\hline Refrigerated Vending Machines ............... & 100 & 32 & 5 & 5 & 9 & 19 & 5 & 3 & 7 & 15 \\
\hline 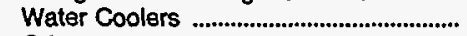 & 100 & 34 & 5 & 5 & 8 & 18 & 4 & 3 & 6 & 16 \\
\hline Other & 100 & 17 & 6 & 4 & 13 & 16 & 5 & 3 & 6 & 30 \\
\hline \multicolumn{11}{|l|}{$\begin{array}{l}\text { ENERGY MANAGEMENT } \\
\text { Occupant Control }\end{array}$} \\
\hline 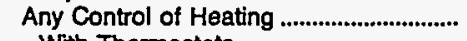 & 100 & 35 & 5 & 4 & 11 & 17 & 5 & 3 & 6 & 14 \\
\hline 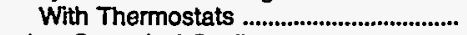 & 100 & 34 & 5 & 4 & 11 & 17 & 5 & 3 & 6 & 15 \\
\hline 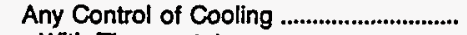 & 100 & 34 & 6 & 4 & 11 & 17 & 5 & 3 & 6 & 14 \\
\hline With Thermostats ...................................... & 100 & 33 & 6 & 4 & 11 & 17 & 5 & 3 & 6 & 15 \\
\hline \multicolumn{11}{|l|}{ Reduced Use During Off-Hours } \\
\hline 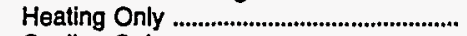 & 100 & 51 & 1 & 3 & 9 & 13 & 2 & 2 & 5 & 12 \\
\hline 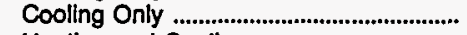 & 100 & 28 & 6 & 4 & 7 & 16 & 5 & 3 & 7 & 25 \\
\hline 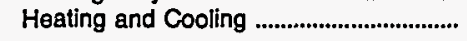 & 100 & 35 & 6 & 5 & 7 & 18 & 5 & 3 & 7 & 14 \\
\hline \multicolumn{11}{|l|}{$\begin{array}{l}\text { Computerized Energy Management } \\
\text { and Control System }\end{array}$} \\
\hline 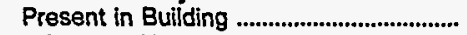 & 100 & 27 & 6 & 7 & 10 & 21 & 6 & 3 & 6 & 14 \\
\hline Controls Heating and Cooling ............... & 100 & 27 & 6 & 7 & 10 & 21 & 6 & 3 & 6 & 14 \\
\hline 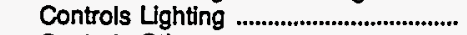 & 100 & 22 & 7 & 5 & 5 & 20 & 15 & 4 & 6 & 16 \\
\hline 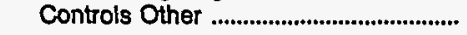 & 100 & 20 & 7 & 6 & 15 & 19 & 4 & 3 & 5 & 22 \\
\hline \multicolumn{11}{|l|}{ Other Energy Management } \\
\hline $\begin{array}{l}\text { Regular HVAC Maintenance ..................... } \\
\text { Participated in Utllity }\end{array}$ & 100 & 33 & 5 & 5 & 9 & 18 & 5 & 3 & 6 & 15 \\
\hline Conservation Program ............................... & 100 & 34 & 5 & 6 & io & 18 & 4 & 3 & 6 & 13 \\
\hline
\end{tabular}

: = Value rounds to zero in the units displayed.

$N C=$ No cases in responding sample.

$Q=$ Data withheld because the Relative Standard Error (RSE) was greater than 50 percent, or data were reported for fewer than 20 buildings.

Notes: - See Glossary for explanation of abbreviations and definitions of terms used in this report. - Because of rounding, data may not sum to totals.

Source: Energy Information Administration, Office of Energy Markets and End Use, Forms ElA-871A through F of the 1989 Commercial Buildings Energy Consumption Survey. 
Table B4. Consumption of Electricity by End Uses, 1989

\begin{tabular}{|c|c|c|c|c|c|c|c|c|c|c|c|}
\hline \multirow{3}{*}{$\begin{array}{c}\text { Buliding } \\
\text { Characteristles } \\
\text { RSE Column Factor: }\end{array}$} & \multicolumn{10}{|c|}{$\begin{array}{l}\text { Electricity Consumption } \\
\text { (trillion Btu) }\end{array}$} & \multirow{3}{*}{$\begin{array}{c}\text { RSE } \\
\text { Row } \\
\text { Factor }\end{array}$} \\
\hline & Total & $\begin{array}{l}\text { Space } \\
\text { Heating }\end{array}$ & Coolling & $\begin{array}{l}\text { Ventl- } \\
\text { ation }\end{array}$ & $\begin{array}{l}\text { Water } \\
\text { Heating }\end{array}$ & Lghting & Cooking| & $\begin{array}{l}\text { Refrig- } \\
\text { eration }\end{array}$ & $\begin{array}{c}\text { Offlce } \\
\text { Equip- } \\
\text { ment }\end{array}$ & Other & \\
\hline & 1.0 & NF & NF & NF & NF & NF & NF & NF & NF & NF & \\
\hline 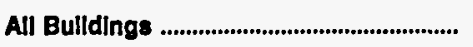 & 2,773 & 96 & 282 & 278 & 24 & 1,023 & 56 & 187 & 379 & 449 & 5.91 \\
\hline 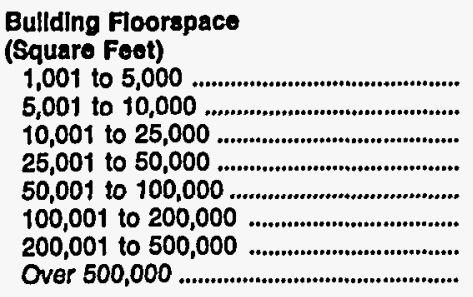 & $\begin{array}{l}326 \\
246 \\
381 \\
331 \\
433 \\
387 \\
366 \\
303\end{array}$ & $\begin{array}{r}15 \\
9 \\
25 \\
0 \\
9 \\
12 \\
7 \\
3\end{array}$ & $\begin{array}{l}44 \\
33 \\
39 \\
34 \\
36 \\
35 \\
35 \\
26\end{array}$ & $\begin{array}{l}29 \\
21 \\
27 \\
26 \\
40 \\
43 \\
50 \\
43\end{array}$ & $\begin{array}{l}8 \\
3 \\
4 \\
2 \\
2 \\
2 \\
2 \\
1\end{array}$ & $\begin{array}{r}96 \\
83 \\
124 \\
127 \\
161 \\
153 \\
151 \\
126\end{array}$ & $\begin{array}{r}15 \\
6 \\
8 \\
6 \\
6 \\
5 \\
5 \\
4\end{array}$ & $\begin{array}{l}33 \\
16 \\
30 \\
27 \\
31 \\
19 \\
15 \\
15\end{array}$ & $\begin{array}{l}52 \\
41 \\
73 \\
46 \\
44 \\
47 \\
39 \\
37\end{array}$ & $\begin{array}{r}33 \\
34 \\
52 \\
46 \\
104 \\
71 \\
62 \\
46\end{array}$ & $\begin{array}{r}6.53 \\
12.33 \\
10.32 \\
10.91 \\
16.23 \\
12.79 \\
17.96 \\
17.56\end{array}$ \\
\hline 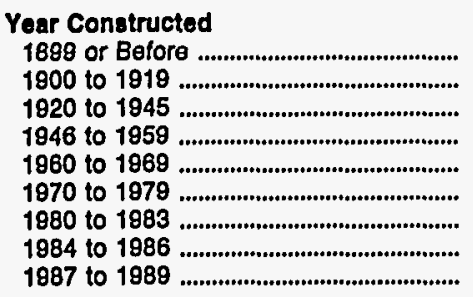 & $\begin{array}{r}25 \\
75 \\
211 \\
379 \\
589 \\
730 \\
295 \\
303 \\
167\end{array}$ & $\begin{array}{r}1 \\
2 \\
Q \\
5 \\
15 \\
31 \\
15 \\
11 \\
4\end{array}$ & $\begin{array}{r}2 \\
5 \\
24 \\
35 \\
60 \\
83 \\
30 \\
29 \\
14\end{array}$ & $\begin{array}{r}3 \\
7 \\
29 \\
42 \\
55 \\
70 \\
23 \\
37 \\
12\end{array}$ & $\begin{array}{l}Q \\
1 \\
1 \\
2 \\
5 \\
8 \\
3 \\
2 \\
1\end{array}$ & $\begin{array}{r}8 \\
29 \\
76 \\
158 \\
219 \\
276 \\
98 \\
104 \\
55\end{array}$ & $\begin{array}{r}1 \\
1 \\
5 \\
6 \\
10 \\
16 \\
7 \\
7 \\
3\end{array}$ & $\begin{array}{r}3 \\
4 \\
14 \\
21 \\
32 \\
51 \\
16 \\
25 \\
21\end{array}$ & $\begin{array}{r}3 \\
10 \\
23 \\
53 \\
72 \\
93 \\
47 \\
50 \\
28\end{array}$ & $\begin{array}{r}4 \\
18 \\
25 \\
56 \\
121 \\
101 \\
56 \\
39 \\
28\end{array}$ & $\begin{array}{r}17.03 \\
16.56 \\
12.34 \\
13.12 \\
15.03 \\
7.28 \\
16.84 \\
12.71 \\
19.81\end{array}$ \\
\hline 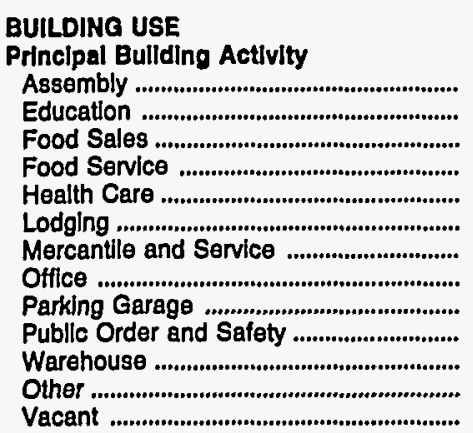 & $\begin{array}{r}186 \\
217 \\
105 \\
113 \\
154 \\
138 \\
550 \\
781 \\
18 \\
29 \\
243 \\
201 \\
39\end{array}$ & $\begin{array}{c}19 \\
10 \\
\cdot \quad \\
1 \\
3 \\
11 \\
12 \\
27 \\
Q \\
Q \\
Q \\
Q \\
Q \\
1\end{array}$ & $\begin{array}{r}25 \\
17 \\
16 \\
19 \\
31 \\
17 \\
51 \\
81 \\
-\quad \\
1 \\
6 \\
14 \\
3\end{array}$ & $\begin{array}{r}25 \\
24 \\
8 \\
13 \\
21 \\
20 \\
26 \\
133 \\
\\
2 \\
3 \\
1\end{array}$ & $\begin{array}{r}1 \\
2 \\
1 \\
3 \\
2 \\
5 \\
4 \\
4 \\
* \\
0 \\
0\end{array}$ & $\begin{array}{r}63 \\
114 \\
19 \\
27 \\
68 \\
47 \\
201 \\
272 \\
8 \\
13 \\
102 \\
76 \\
13\end{array}$ & $\begin{array}{r}1 \\
1 \\
9 \\
17 \\
5 \\
14 \\
4 \\
3 \\
* \quad \\
* \\
1 \\
1\end{array}$ & $\begin{array}{r}11 \\
10 \\
45 \\
16 \\
6 \\
16 \\
12 \\
35 \\
1 \\
2 \\
24 \\
7 \\
2\end{array}$ & $\begin{array}{r}6 \\
10 \\
1 \\
2 \\
5 \\
1 \\
115 \\
165 \\
1 \\
1 \\
57 \\
0 \\
2\end{array}$ & $\begin{array}{r}35 \\
28 \\
7 \\
15 \\
12 \\
6 \\
124 \\
62 \\
7 \\
8 \\
39 \\
0 \\
18\end{array}$ & $\begin{array}{r}17.84 \\
9.30 \\
22.55 \\
11.37 \\
13.95 \\
11.61 \\
10.77 \\
6.40 \\
25.81 \\
28.04 \\
18.91 \\
41.44 \\
19.68\end{array}$ \\
\hline 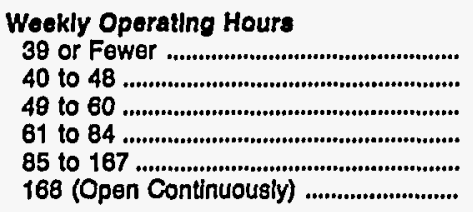 & $\begin{array}{r}71 \\
440 \\
478 \\
522 \\
485 \\
779\end{array}$ & $\begin{array}{r}5 \\
16 \\
22 \\
14 \\
12 \\
27\end{array}$ & $\begin{array}{r}7 \\
47 \\
44 \\
54 \\
47 \\
83\end{array}$ & $\begin{array}{l}10 \\
43 \\
52 \\
52 \\
38 \\
83\end{array}$ & $\begin{array}{l}1 \\
3 \\
3 \\
3 \\
4 \\
9\end{array}$ & $\begin{array}{r}23 \\
158 \\
169 \\
189 \\
186 \\
289\end{array}$ & $\begin{array}{r}1 \\
2 \\
3 \\
7 \\
14 \\
28\end{array}$ & $\begin{array}{r}6 \\
27 \\
24 \\
31 \\
44 \\
54\end{array}$ & $\begin{array}{l}7 \\
71 \\
93 \\
71 \\
56 \\
81\end{array}$ & $\begin{array}{r}12 \\
73 \\
69 \\
100 \\
72 \\
123\end{array}$ & $\begin{array}{r}11.94 \\
9.57 \\
7.93 \\
10.12 \\
10.27 \\
13.40\end{array}$ \\
\hline 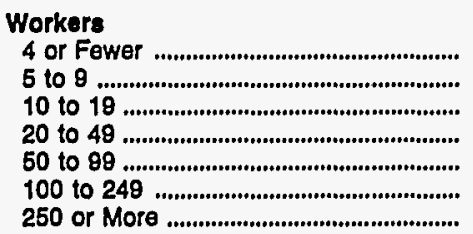 & $\begin{array}{l}294 \\
258 \\
238 \\
401 \\
348 \\
478 \\
758\end{array}$ & $\begin{array}{r}15 \\
16 \\
11 \\
14 \\
17 \\
9 \\
13\end{array}$ & $\begin{array}{l}34 \\
30 \\
30 \\
40 \\
36 \\
42 \\
71\end{array}$ & $\begin{array}{r}28 \\
21 \\
20 \\
27 \\
25 \\
43 \\
113\end{array}$ & $\begin{array}{l}6 \\
4 \\
3 \\
4 \\
2 \\
3 \\
2\end{array}$ & $\begin{array}{r}88 \\
83 \\
74 \\
156 \\
133 \\
201 \\
288\end{array}$ & $\begin{array}{r}11 \\
8 \\
8 \\
9 \\
7 \\
6 \\
8\end{array}$ & $\begin{array}{l}30 \\
17 \\
20 \\
33 \\
28 \\
29 \\
31\end{array}$ & $\begin{array}{l}44 \\
42 \\
42 \\
56 \\
39 \\
58 \\
99\end{array}$ & $\begin{array}{r}39 \\
37 \\
31 \\
61 \\
60 \\
87 \\
133\end{array}$ & $\begin{array}{r}5.56 \\
8.81 \\
11.44 \\
9.28 \\
10.76 \\
15.02 \\
17.20\end{array}$ \\
\hline
\end{tabular}

See footnotes at end of table. 


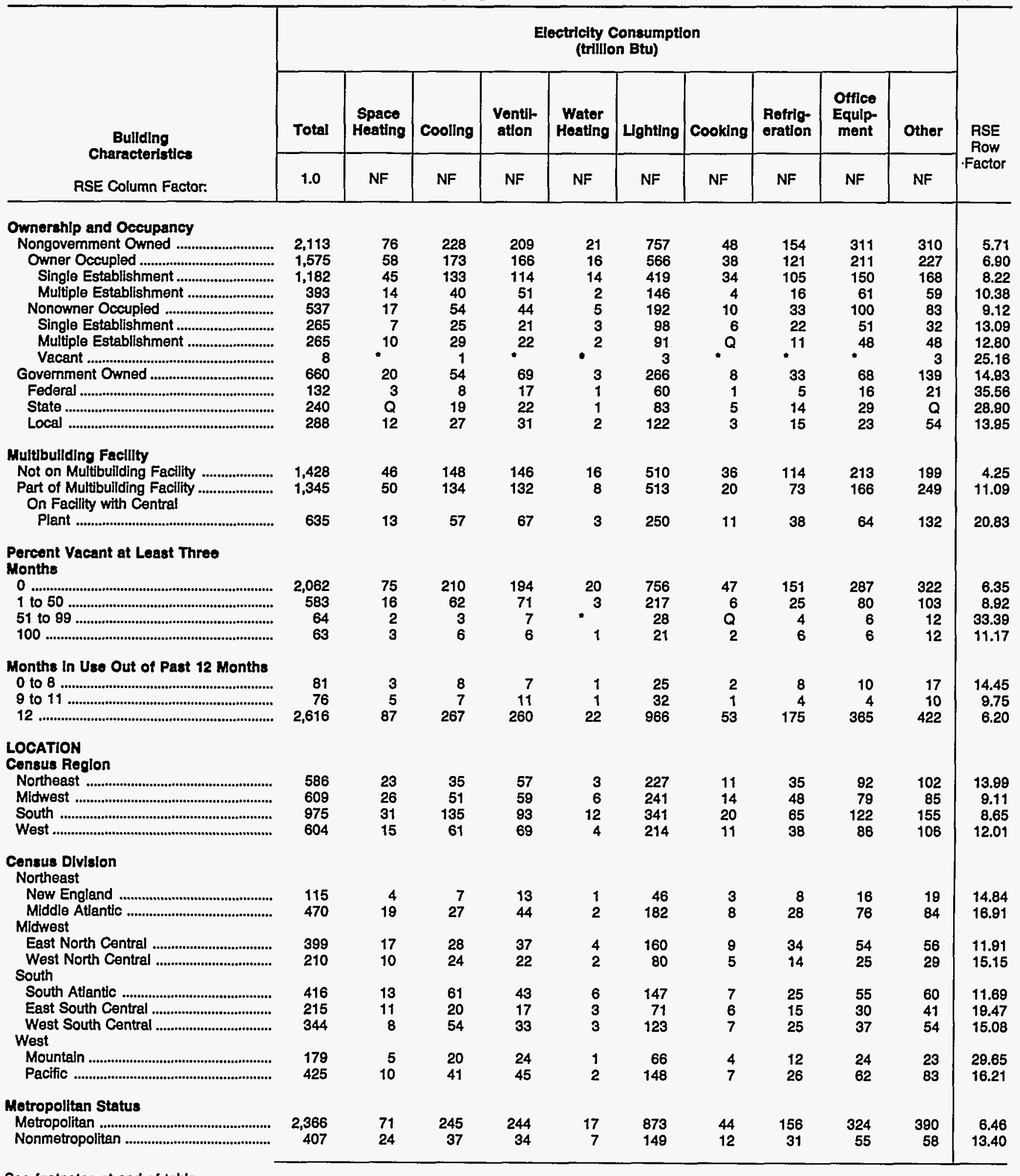

See footnotes at end of table. 
Table B4. Consumption of Electricity by End Uses, 1989 (Continued)

\begin{tabular}{|c|c|c|c|c|c|c|c|c|c|c|c|}
\hline \multirow{3}{*}{$\begin{array}{c}\text { Bullding } \\
\text { Characterlatlos } \\
\text { RSE Column Factor: }\end{array}$} & \multicolumn{10}{|c|}{$\begin{array}{l}\text { Electricity Consumption } \\
\text { (trillion Btu) }\end{array}$} & \multirow{3}{*}{$\begin{array}{c}\text { RSE } \\
\text { Row } \\
\text { Factor }\end{array}$} \\
\hline & Total & $\begin{array}{l}\text { Space } \\
\text { Heating }\end{array}$ & Cooling & $\begin{array}{l}\text { Ventl- } \\
\text { ation }\end{array}$ & $\begin{array}{c}\text { Water } \\
\text { Heating }\end{array}$ & LghtIng & Cooklng & $\begin{array}{l}\text { Refrig- } \\
\text { eration }\end{array}$ & $\begin{array}{l}\text { Offlce } \\
\text { Equip- } \\
\text { ment }\end{array}$ & Other & \\
\hline & 1.0 & NF & NF & NF & NF & NF & NF & $N F$ & NF & NF & \\
\hline 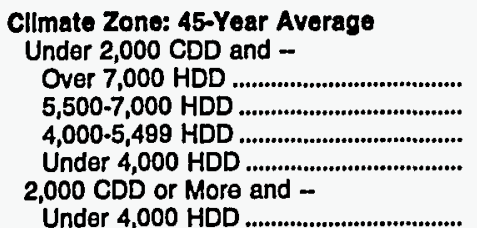 & $\begin{array}{l}211 \\
668 \\
706 \\
663 \\
525\end{array}$ & $\begin{array}{l}10 \\
25 \\
33 \\
17\end{array}$ & $\begin{array}{l}10 \\
45 \\
59 \\
70\end{array}$ & $\begin{array}{l}16 \\
70 \\
72 \\
67 \\
52\end{array}$ & $\begin{array}{l}2 \\
5 \\
7 \\
5\end{array}$ & $\begin{array}{r}84 \\
266 \\
279 \\
225\end{array}$ & $\begin{array}{r}6 \\
16 \\
11 \\
12\end{array}$ & $\begin{array}{l}23 \\
47 \\
42 \\
38 \\
37\end{array}$ & $\begin{array}{r}30 \\
93 \\
102 \\
93 \\
60\end{array}$ & $\begin{array}{r}31 \\
102 \\
101 \\
135 \\
80\end{array}$ & $\begin{array}{l}17.08 \\
14.48 \\
10.12 \\
16.48 \\
11.41\end{array}$ \\
\hline 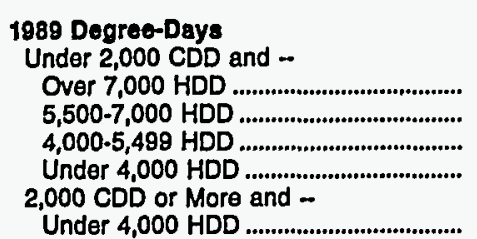 & $\begin{array}{l}286 \\
852 \\
539 \\
594 \\
503\end{array}$ & $\begin{array}{l}10 \\
34 \\
28 \\
13 \\
11\end{array}$ & $\begin{array}{l}14 \\
67 \\
38 \\
67\end{array}$ & $\begin{array}{l}23 \\
91 \\
54 \\
60\end{array}$ & $\begin{array}{l}2 \\
7 \\
5 \\
4\end{array}$ & $\begin{array}{l}117 \\
335 \\
212 \\
197\end{array}$ & $\begin{array}{r}8 \\
18 \\
9 \\
10\end{array}$ & $\begin{array}{l}29 \\
55 \\
34 \\
34\end{array}$ & $\begin{array}{r}38 \\
119 \\
81 \\
83\end{array}$ & $\begin{array}{r}44 \\
126 \\
79 \\
126\end{array}$ & $\begin{array}{l}15.40 \\
13.17 \\
13.41 \\
16.04\end{array}$ \\
\hline $\begin{array}{l}\text { STRUCTURE } \\
\text { Floore } \\
1 \\
2 \\
3 \\
4 \text { to } 6 \\
7 \text { or More }\end{array}$ & $\begin{array}{l}922 \\
793 \\
320 \\
342 \\
396\end{array}$ & $\begin{array}{r}31 \\
29 \\
17 \\
9 \\
10\end{array}$ & $\begin{array}{r}110 \\
69 \\
29 \\
34 \\
39\end{array}$ & $\begin{array}{l}71 \\
58 \\
35 \\
42 \\
73\end{array}$ & $\begin{array}{r}11 \\
5 \\
3 \\
2 \\
2\end{array}$ & $\begin{array}{l}322 \\
298 \\
107 \\
144 \\
151\end{array}$ & $\begin{array}{r}23 \\
13 \\
5 \\
8 \\
7\end{array}$ & $\begin{array}{l}74 \\
59 \\
15 \\
20 \\
19\end{array}$ & $\begin{array}{r}142 \\
114 \\
38 \\
35 \\
51\end{array}$ & $\begin{array}{r}138 \\
148 \\
Q \\
47 \\
44\end{array}$ & $\begin{array}{r}6.91 \\
10.14 \\
21.45 \\
13.89 \\
11.85\end{array}$ \\
\hline 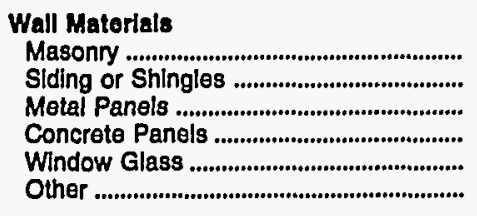 & $\begin{array}{r}1,740 \\
150 \\
246 \\
397 \\
146 \\
94\end{array}$ & $\begin{array}{r}54 \\
9 \\
10 \\
15 \\
6 \\
2\end{array}$ & $\begin{array}{r}191 \\
15 \\
21 \\
36 \\
12 \\
6\end{array}$ & $\begin{array}{r}168 \\
12 \\
19 \\
38 \\
26 \\
14\end{array}$ & $\begin{array}{r}16 \\
3 \\
2 \\
2 \\
1 \\
:\end{array}$ & $\begin{array}{r}634 \\
56 \\
97 \\
144 \\
54 \\
39\end{array}$ & $\begin{array}{r}40 \\
4 \\
2 \\
6 \\
0 \\
1\end{array}$ & $\begin{array}{r}127 \\
10 \\
15 \\
24 \\
7 \\
4\end{array}$ & $\begin{array}{r}230 \\
22 \\
42 \\
46 \\
24 \\
15\end{array}$ & $\begin{array}{r}279 \\
19 \\
40 \\
84 \\
13 \\
13\end{array}$ & $\begin{array}{r}6.33 \\
11.52 \\
19.67 \\
15.84 \\
22.39 \\
22.18\end{array}$ \\
\hline 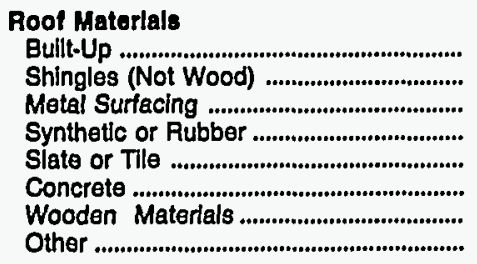 & $\begin{array}{r}1,484 \\
341 \\
314 \\
416 \\
70 \\
71 \\
29 \\
48\end{array}$ & $\begin{array}{r}48 \\
14 \\
10 \\
16 \\
3 \\
2 \\
1 \\
Q\end{array}$ & $\begin{array}{r}154 \\
40 \\
21 \\
40 \\
8 \\
6 \\
4 \\
7\end{array}$ & $\begin{array}{r}152 \\
35 \\
17 \\
45 \\
8 \\
12 \\
4 \\
0\end{array}$ & $\begin{array}{r}12 \\
5 \\
3 \\
2 \\
1 \\
Q \\
0 \\
Q\end{array}$ & $\begin{array}{r}556 \\
119 \\
118 \\
151 \\
23 \\
28 \\
9 \\
19\end{array}$ & $\begin{array}{r}29 \\
10 \\
4 \\
6 \\
3 \\
1 \\
1 \\
1\end{array}$ & $\begin{array}{r}92 \\
31 \\
20 \\
30 \\
6 \\
3 \\
2 \\
2\end{array}$ & $\begin{array}{r}191 \\
45 \\
59 \\
57 \\
Q \\
10 \\
3 \\
Q\end{array}$ & $\begin{array}{r}249 \\
43 \\
61 \\
68 \\
9 \\
9 \\
4 \\
5\end{array}$ & $\begin{array}{r}8.05 \\
8.20 \\
15.58 \\
15.18 \\
23.25 \\
32.42 \\
18.89 \\
38.31\end{array}$ \\
\hline $\begin{array}{l}\text { Bulleding Shell Conservation } \\
\text { Features (Solely or In Combinatlon) } \\
\text { Roof or Celling Insulation ......................... } \\
\text { Wall Insulation ............................................ } \\
\text { Storm or Multiple Glazing ...................... } \\
\text { Tinted, Reflective, or Shading }\end{array}$ & $\begin{array}{l}2,201 \\
1,547 \\
1,225\end{array}$ & $\begin{array}{l}79 \\
56 \\
43\end{array}$ & $\begin{array}{l}234 \\
162 \\
123\end{array}$ & $\begin{array}{l}223 \\
148 \\
128\end{array}$ & $\begin{array}{l}19 \\
14 \\
12\end{array}$ & $\begin{array}{l}820 \\
585 \\
455\end{array}$ & $\begin{array}{l}45 \\
30 \\
28\end{array}$ & $\begin{array}{r}149 \\
105 \\
92\end{array}$ & $\begin{array}{l}306 \\
216 \\
180\end{array}$ & $\begin{array}{l}326 \\
229 \\
163\end{array}$ & $\begin{array}{l}5.92 \\
7.36 \\
7.24\end{array}$ \\
\hline 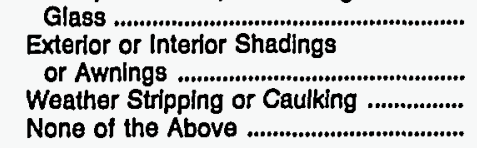 & $\begin{array}{r}1,381 \\
2,257 \\
134\end{array}$ & $\begin{array}{r}41 \\
80 \\
4\end{array}$ & $\begin{array}{r}140 \\
230 \\
12\end{array}$ & $\begin{array}{r}149 \\
229 \\
12\end{array}$ & $\begin{array}{r}12 \\
20 \\
1\end{array}$ & $\begin{array}{r}490 \\
496 \\
829 \\
51\end{array}$ & $\begin{array}{r}20 \\
27 \\
44 \\
3\end{array}$ & $\begin{array}{r}76 \\
82 \\
145 \\
11\end{array}$ & $\begin{array}{r}189 \\
195 \\
310 \\
19\end{array}$ & $\begin{array}{r}198 \\
239 \\
371 \\
20\end{array}$ & $\begin{array}{r}7.35 \\
8.24 \\
6.64 \\
10.78\end{array}$ \\
\hline
\end{tabular}

See footnotes at end of table. 


\begin{tabular}{|c|c|c|c|c|c|c|c|c|c|c|c|}
\hline \multirow{3}{*}{$\begin{array}{c}\text { Bullding } \\
\text { Characteristlcs } \\
\text { RSE Column Factor: }\end{array}$} & \multicolumn{10}{|c|}{$\begin{array}{l}\text { Electriclty Consumption } \\
\text { (trillion Btu) }\end{array}$} & \multirow{3}{*}{$\begin{array}{c}\text { RSE } \\
\text { Row } \\
\text { Factor }\end{array}$} \\
\hline & Total & $\begin{array}{l}\text { Space } \\
\text { Heating }\end{array}$ & Coolling & $\begin{array}{l}\text { Ventll- } \\
\text { ation }\end{array}$ & $\begin{array}{c}\text { Water } \\
\text { Heating }\end{array}$ & Lighting & Cooking & $\begin{array}{l}\text { Refrig- } \\
\text { eration }\end{array}$ & $\begin{array}{c}\text { Ottice } \\
\text { Equip- } \\
\text { ment }\end{array}$ & Other & \\
\hline & 1.0 & NF & NF & NF & NF & NF & NF & NF & NF & NF & \\
\hline 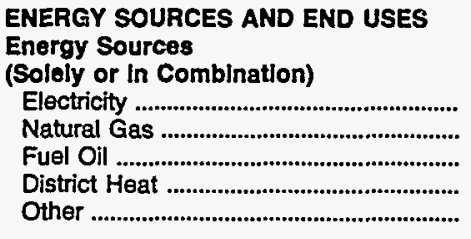 & $\begin{array}{r}2,773 \\
1,824 \\
662 \\
444 \\
402\end{array}$ & $\begin{array}{l}96 \\
29 \\
10 \\
Q \\
9\end{array}$ & $\begin{array}{r}282 \\
187 \\
66 \\
34 \\
30\end{array}$ & $\begin{array}{r}278 \\
195 \\
85 \\
56 \\
34\end{array}$ & $\begin{array}{r}24 \\
10 \\
5 \\
Q \\
4\end{array}$ & $\begin{array}{r}1,023 \\
689 \\
263 \\
165 \\
152\end{array}$ & $\begin{array}{r}56 \\
41 \\
11 \\
8 \\
9\end{array}$ & $\begin{array}{r}187 \\
122 \\
40 \\
21 \\
33\end{array}$ & $\begin{array}{r}379 \\
237 \\
91 \\
46 \\
42\end{array}$ & $\begin{array}{r}449 \\
313 \\
90 \\
98 \\
89\end{array}$ & $\begin{array}{r}5.91 \\
8.39 \\
15.56 \\
20.92 \\
15.70\end{array}$ \\
\hline 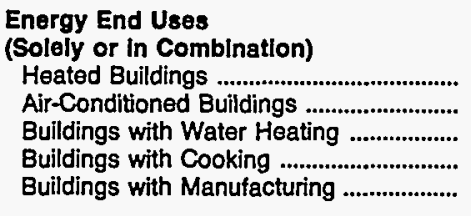 & $\begin{array}{r}2,676 \\
2,555 \\
2,602 \\
1,331 \\
291\end{array}$ & $\begin{array}{l}96 \\
91 \\
90 \\
32 \\
Q\end{array}$ & $\begin{array}{r}267 \\
282 \\
263 \\
142 \\
17\end{array}$ & $\begin{array}{r}268 \\
256 \\
263 \\
150 \\
15\end{array}$ & $\begin{array}{r}23 \\
23 \\
24 \\
12 \\
1\end{array}$ & $\begin{array}{l}993 \\
933 \\
958 \\
502 \\
112\end{array}$ & $\begin{array}{r}54 \\
51 \\
54 \\
41 \\
1\end{array}$ & $\begin{array}{r}179 \\
172 \\
179 \\
96 \\
19\end{array}$ & $\begin{array}{r}369 \\
342 \\
347 \\
130 \\
38\end{array}$ & $\begin{array}{r}427 \\
406 \\
422 \\
226 \\
81\end{array}$ & $\begin{array}{r}5.99 \\
5.78 \\
6.17 \\
9.79 \\
17.76\end{array}$ \\
\hline 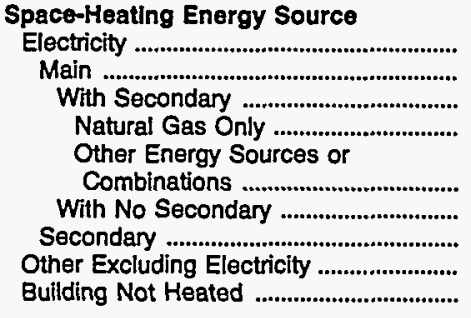 & $\begin{array}{r}1,039 \\
800 \\
118 \\
57 \\
54 \\
682 \\
239 \\
1,637 \\
97\end{array}$ & $\begin{array}{r}96 \\
75 \\
9 \\
3 \\
6 \\
66 \\
21 \\
\text { NC } \\
\text { NC }\end{array}$ & $\begin{array}{r}112 \\
92 \\
13 \\
6 \\
5 \\
78 \\
20 \\
155 \\
15\end{array}$ & $\begin{array}{r}83 \\
68 \\
10 \\
3 \\
0 \\
0 \\
58 \\
15 \\
185 \\
10\end{array}$ & $\begin{array}{r}15 \\
13 \\
2 \\
- \\
\\
2 \\
11 \\
1 \\
8 \\
2\end{array}$ & $\begin{array}{r}355 \\
263 \\
37 \\
18 \\
18 \\
226 \\
92 \\
637 \\
30\end{array}$ & $\begin{array}{r}20 \\
17 \\
3 \\
Q \\
1 \\
14 \\
3 \\
34 \\
2\end{array}$ & $\begin{array}{r}70 \\
59 \\
Q \\
Q \\
2 \\
46 \\
11 \\
109 \\
8\end{array}$ & $\begin{array}{r}144 \\
120 \\
14 \\
6 \\
\\
0 \\
106 \\
24 \\
225 \\
10\end{array}$ & $\begin{array}{r}144 \\
92 \\
16 \\
8 \\
7 \\
7 \\
76 \\
52 \\
284 \\
21\end{array}$ & $\begin{array}{r}6.02 \\
5.83 \\
21.04 \\
30.58 \\
38.11 \\
5.36 \\
15.24 \\
8.50 \\
22.85\end{array}$ \\
\hline 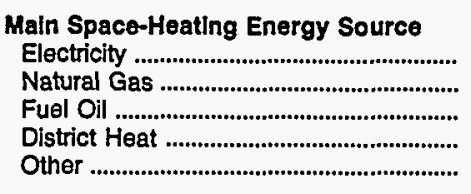 & $\begin{array}{r}800 \\
1,287 \\
182 \\
349 \\
62\end{array}$ & $\begin{array}{r}75 \\
9 \\
1 \\
Q \\
Q\end{array}$ & $\begin{array}{r}92 \\
136 \\
11 \\
25 \\
3\end{array}$ & $\begin{array}{r}68 \\
134 \\
16 \\
49 \\
5\end{array}$ & $\begin{array}{r}13 \\
6 \\
2 \\
1 \\
1\end{array}$ & $\begin{array}{r}263 \\
489 \\
72 \\
139 \\
23\end{array}$ & $\begin{array}{r}17 \\
26 \\
4 \\
7 \\
Q\end{array}$ & $\begin{array}{l}59 \\
81 \\
11 \\
18 \\
Q\end{array}$ & $\begin{array}{r}120 \\
169 \\
35 \\
37 \\
9\end{array}$ & $\begin{array}{r}92 \\
237 \\
30 \\
60 \\
8\end{array}$ & $\begin{array}{r}5.83 \\
8.70 \\
18.86 \\
17.80 \\
41.49\end{array}$ \\
\hline 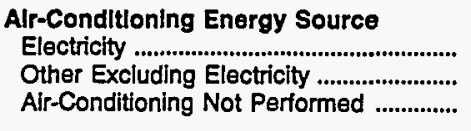 & $\begin{array}{r}2,373 \\
182 \\
218\end{array}$ & $\begin{array}{c}86 \\
Q \\
5\end{array}$ & $\begin{array}{l}282 \\
\text { NC } \\
\text { NC }\end{array}$ & $\begin{array}{r}230 \\
26 \\
22\end{array}$ & $\begin{array}{r}22 \\
1 \\
1\end{array}$ & $\begin{array}{r}848 \\
85 \\
90\end{array}$ & $\begin{array}{r}47 \\
5 \\
4\end{array}$ & $\begin{array}{r}161 \\
11 \\
15\end{array}$ & $\begin{array}{r}321 \\
21 \\
37\end{array}$ & $\begin{array}{r}376 \\
30 \\
43\end{array}$ & $\begin{array}{r}5.81 \\
17.62 \\
17.62\end{array}$ \\
\hline 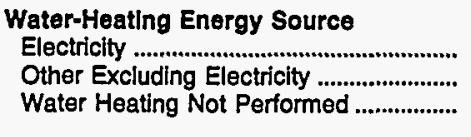 & $\begin{array}{r}1,135 \\
1,466 \\
171\end{array}$ & $\begin{array}{r}65 \\
25 \\
6\end{array}$ & $\begin{array}{r}122 \\
141 \\
18\end{array}$ & $\begin{array}{r}100 \\
163 \\
15\end{array}$ & $\begin{array}{l}24 \\
\text { NC } \\
\text { NC }\end{array}$ & $\begin{array}{r}381 \\
577 \\
65\end{array}$ & $\begin{array}{r}15 \\
39 \\
1\end{array}$ & $\begin{array}{r}74 \\
105 \\
8\end{array}$ & $\begin{array}{r}175 \\
172 \\
32\end{array}$ & $\begin{array}{r}178 \\
245 \\
26\end{array}$ & $\begin{array}{l}6.36 \\
8.57 \\
9.30\end{array}$ \\
\hline 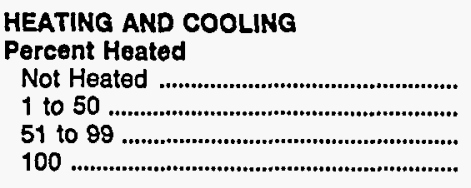 & $\begin{array}{r}98 \\
210 \\
496 \\
1,969\end{array}$ & $\begin{array}{r}Q \\
4 \\
13 \\
79\end{array}$ & $\begin{array}{r}15 \\
15 \\
51 \\
201\end{array}$ & $\begin{array}{r}10 \\
11 \\
54 \\
203\end{array}$ & $\begin{array}{r}2 \\
2 \\
4 \\
17\end{array}$ & $\begin{array}{r}30 \\
76 \\
178 \\
739\end{array}$ & $\begin{array}{r}2 \\
3 \\
9 \\
42\end{array}$ & $\begin{array}{r}8 \\
24 \\
28 \\
127\end{array}$ & $\begin{array}{r}10 \\
41 \\
77 \\
250\end{array}$ & $\begin{array}{r}21 \\
33 \\
83 \\
310\end{array}$ & $\begin{array}{r}22.55 \\
15.51 \\
11.17 \\
7.68\end{array}$ \\
\hline $\begin{array}{l}\text { Percent Cooled } \\
\text { Not Cooled } \\
1 \text { to } 50 \text { to } 99 \\
100\end{array}$ & $\begin{array}{r}218 \\
461 \\
783 \\
1,311\end{array}$ & $\begin{array}{r}5 \\
16 \\
22 \\
53\end{array}$ & $\begin{array}{l}\text { NC } \\
31 \\
91 \\
161\end{array}$ & $\begin{array}{r}22 \\
29 \\
88 \\
138\end{array}$ & $\begin{array}{r}1 \\
3 \\
7 \\
13\end{array}$ & $\begin{array}{r}90 \\
172 \\
295 \\
466\end{array}$ & $\begin{array}{r}4 \\
8 \\
16 \\
28\end{array}$ & $\begin{array}{l}15 \\
34 \\
55 \\
83\end{array}$ & $\begin{array}{r}37 \\
75 \\
101 \\
166\end{array}$ & $\begin{array}{r}43 \\
93 \\
109 \\
204\end{array}$ & $\begin{array}{r}17.62 \\
9.26 \\
7.78 \\
9.51\end{array}$ \\
\hline
\end{tabular}

See footnotes at end of table. 
Table B4. Consumption of Electricity by End Use, 1989 (Continued)

\begin{tabular}{|c|c|c|c|c|c|c|c|c|c|c|c|}
\hline \multirow{3}{*}{$\begin{array}{c}\text { Bullding } \\
\text { Characteristics } \\
\text { RSE Column Factor: }\end{array}$} & \multicolumn{10}{|c|}{$\begin{array}{l}\text { Electricity Consumption } \\
\text { (trillion Btu) }\end{array}$} & \multirow{3}{*}{$\begin{array}{c}\text { RSE } \\
\text { Row } \\
\text { Factor }\end{array}$} \\
\hline & Total & $\begin{array}{l}\text { Space } \\
\text { Heating }\end{array}$ & Cooling & $\begin{array}{l}\text { Ventil- } \\
\text { ation }\end{array}$ & $\begin{array}{l}\text { Water } \\
\text { Heatling }\end{array}$ & Lighting & Cooking & $\begin{array}{l}\text { Refrig- } \\
\text { eration }\end{array}$ & $\begin{array}{l}\text { Office } \\
\text { Equip- } \\
\text { ment }\end{array}$ & Other & \\
\hline & 1.0 & NF & NF & NF & NF & NF & NF & NF & NF & NF & \\
\hline \multicolumn{12}{|l|}{$\begin{array}{l}\text { Heating Equipment } \\
\text { (Solely or in Combination) }\end{array}$} \\
\hline 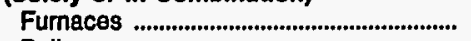 & 608 & 22 & 55 & 45 & 6 & 219 & 15 & 52 & 99 & 95 & 8.80 \\
\hline 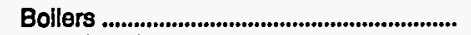 & 874 & 19 & 85 & 108 & 5 & 354 & 14 & 56 & 103 & 131 & 9.13 \\
\hline Individual Space Heaters .............................. & 942 & 50 & 80 & 82 & 9 & 358 & 15 & 54 & 133 & 160 & 9.04 \\
\hline Packaged Heating Units .............................. & 878 & 31 & 100 & 68 & 7 & 316 & 18 & 50 & 117 & 171 & 10.89 \\
\hline 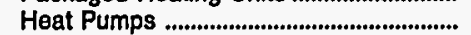 & 426 & 26 & 49 & 43 & 4 & 148 & 8 & 31 & 57 & 60 & 10.47 \\
\hline 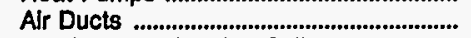 & 2,004 & 58 & 205 & 205 & 15 & 753 & 37 & 125 & 275 & 330 & 7.44 \\
\hline Heating or Reheating Coils ........................ & 1,040 & 28 & 97 & 127 & 5 & 401 & 16 & 52 & 123 & 191 & 13.64 \\
\hline $\begin{array}{l}\text { Fan-Coil Units ............................................. } \\
\text { Steam or Hot Water Radiators }\end{array}$ & 682 & $\mathbf{Q}$ & 59 & 79 & 3 & 273 & 10 & 34 & 77 & 129 & 15.07 \\
\hline 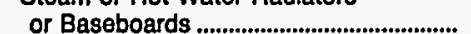 & 603 & 12 & 45 & 77 & 3 & 256 & 12 & 34 & 71 & 93 & 10.51 \\
\hline Other & 117 & $\bar{Q}$ & 14 & $\mathbf{Q}$ & 1 & 41 & 2 & 9 & 14 & 14 & 29.18 \\
\hline \multicolumn{12}{|l|}{$\begin{array}{l}\text { Cooling Equlpment } \\
\text { (Solely or In Combination) }\end{array}$} \\
\hline 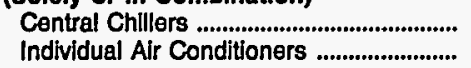 & $\begin{array}{l}922 \\
754\end{array}$ & $\begin{array}{l}28 \\
28\end{array}$ & $\begin{array}{r}101 \\
75\end{array}$ & $\begin{array}{r}132 \\
70\end{array}$ & $\begin{array}{l}5 \\
9\end{array}$ & $\begin{array}{l}344 \\
269\end{array}$ & $\begin{array}{l}13 \\
15\end{array}$ & $\begin{array}{l}42 \\
52\end{array}$ & $\begin{array}{r}108 \\
87\end{array}$ & $\begin{array}{l}150 \\
150\end{array}$ & $\begin{array}{l}13.05 \\
10.36\end{array}$ \\
\hline Packaged Coolling Units .................................. & 1,804 & 53 & 203 & 170 & 15 & 655 & 37 & 122 & 244 & 305 & 7.32 \\
\hline Heat Pumps …………................................................. & 467 & 26 & 48 & 40 & 5 & 152 & 7 & 30 & 64 & 96 & 16.32 \\
\hline 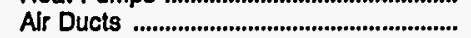 & 1,877 & 63 & 205 & 195 & 14 & 696 & 36 & 116 & 246 & 305 & 7.31 \\
\hline 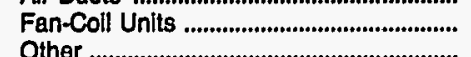 & $\begin{array}{r}753 \\
63\end{array}$ & 22 & $\begin{array}{r}76 \\
8\end{array}$ & $\begin{array}{r}108 \\
5\end{array}$ & 3 & $\begin{array}{r}288 \\
29\end{array}$ & 10 & 35 & 89 & $\begin{array}{r}121 \\
8\end{array}$ & $\begin{array}{l}14.29 \\
33.67\end{array}$ \\
\hline \multicolumn{12}{|l|}{ Year Maln Central Chiller Installed } \\
\hline 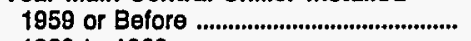 & 91 & Q & 11 & 16 & 1 & 32 & 1 & 4 & 10 & 8 & 24.98 \\
\hline 1960 to 1969 & 252 & 3 & 26 & 29 & & 90 & 2 & 9 & 26 & $\mathbf{Q}$ & 31.68 \\
\hline 1970 to 1979 & 231 & 5 & 24 & 32 & 2 & 89 & 3 & 10 & 29 & 36 & 15.03 \\
\hline 1980 to $1986 \ldots$ & 245 & 9 & 28 & 41 & 1 & 93 & 4 & 12 & 30 & 26 & 21.11 \\
\hline 1987 to 1889 & 103 & 2 & 12 & 14 & $\mathbf{Q}$ & 40 & 2 & 6 & 12 & 13 & 22.77 \\
\hline \multicolumn{12}{|l|}{$\begin{array}{l}\text { Year Packaged Coollng System } \\
\text { Installed }\end{array}$} \\
\hline 1959 or Before & 79 & 1 & 8 & 9 & - & 36 & 1 & 3 & 9 & 11 & 18.32 \\
\hline 1960 to 1869 & 276 & 6 & 25 & 21 & 1 & 98 & 3 & 13 & 36 & $\mathbf{Q}$ & 25.88 \\
\hline 1970 to 1979 & 547 & 24 & 67 & 53 & 6 & 205 & 12 & 36 & 73 & 73 & 8.37 \\
\hline 1980 to 1986 & 599 & 19 & 64 & 55 & 5 & 213 & 14 & 47 & 88 & 93 & 10.44 \\
\hline 1987 to 1989 & 303 & 5 & 37 & 31 & 2 & 104 & 7 & 23 & 38 & 56 & 10.68 \\
\hline \multicolumn{12}{|l|}{$\begin{array}{l}\text { Computer Area with Separato } \\
\text { Alr-Conditloning System }\end{array}$} \\
\hline 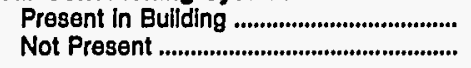 & $\begin{array}{l}1,194 \\
1,579\end{array}$ & $\begin{array}{l}24 \\
72\end{array}$ & $\begin{array}{l}114 \\
168\end{array}$ & $\begin{array}{l}145 \\
133\end{array}$ & $\begin{array}{r}5 \\
20\end{array}$ & $\begin{array}{l}452 \\
570\end{array}$ & $\begin{array}{l}13 \\
43\end{array}$ & $\begin{array}{r}63 \\
124\end{array}$ & $\begin{array}{l}181 \\
198\end{array}$ & $\begin{array}{l}198 \\
250\end{array}$ & $\begin{array}{r}10.59 \\
5.77\end{array}$ \\
\hline \multicolumn{12}{|l|}{ LIGHTING AND REFRIGERATION } \\
\hline 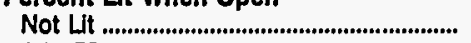 & & $Q$ & - & $\cdot$ & - & 2 & - & 1 & $\mathbf{Q}$ & 3 & 30.06 \\
\hline 1 to 50 & 192 & 14 & 20 & 22 & 4 & 48 & 5 & 15 & 32 & 35 & 9.45 \\
\hline 51 to 89 & 813 & 22 & 80 & 85 & 6 & 293 & 12 & 43 & 114 & 157 & 9.92 \\
\hline 100 & 1,760 & 59 & 182 & 171 & 14 & 680 & 39 & 129 & 232 & 253 & 7.78 \\
\hline Porcent Lit When Closed & & & & & & & & & & & \\
\hline 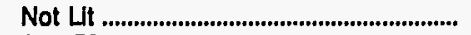 & 947 & 52 & 100 & 85 & 10 & 309 & 22 & 63 & 135 & 172 & 8.73 \\
\hline 1 to 50 & 1,566 & 42 & 149 & 160 & 11 & 613 & 25 & 102 & 220 & 244 & 6.46 \\
\hline 51 to 99 & $\begin{array}{r}203 \\
57\end{array}$ & $Q^{2}$ & $\begin{array}{l}23 \\
10\end{array}$ & $\begin{array}{r}27 \\
7\end{array}$ & $Q^{2}$ & $\begin{array}{l}81 \\
20\end{array}$ & $\stackrel{6}{Q}$ & $\begin{array}{c}13 \\
Q\end{array}$ & $\begin{array}{r}22 \\
2\end{array}$ & $\begin{array}{r}28 \\
5\end{array}$ & $\begin{array}{l}25.13 \\
36.11\end{array}$ \\
\hline $\begin{array}{l}\text { Llghting Equlpment } \\
\text { (Solely or In Combination) }\end{array}$ & & & & & & & & & & & \\
\hline 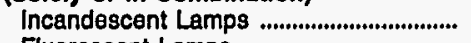 & 1,747 & 62 & 180 & 183 & 15 & 685 & 38 & 115 & 206 & 265 & 7.40 \\
\hline Fluorescent Lamps ......................................... & 2,735 & 84 & $\begin{array}{r}279 \\
85\end{array}$ & $\begin{array}{r}274 \\
95\end{array}$ & 24 & 1,008 & 55 & 185 & 375 & 440 & 5.93 \\
\hline High-Intensity Discharge Lamps .............. & $\begin{array}{r}982 \\
28\end{array}$ & $\begin{array}{l}31 \\
0\end{array}$ & $\begin{array}{r}85 \\
4\end{array}$ & $\begin{array}{r}95 \\
4\end{array}$ & .5 & $\begin{array}{r}396 \\
12\end{array}$ & $0^{73}$ & 63 & $\begin{array}{r}122 \\
3\end{array}$ & $\begin{array}{r}73 \\
3\end{array}$ & $\begin{array}{l}10.04 \\
24.45\end{array}$ \\
\hline HIgh-Efficlency Ballasts .............................. & 1,415 & 53 & 141 & 152 & 11 &, 490 & 28 & 99 & 213 & 228 & 8.82 \\
\hline
\end{tabular}

See tootnotes at end of table. 


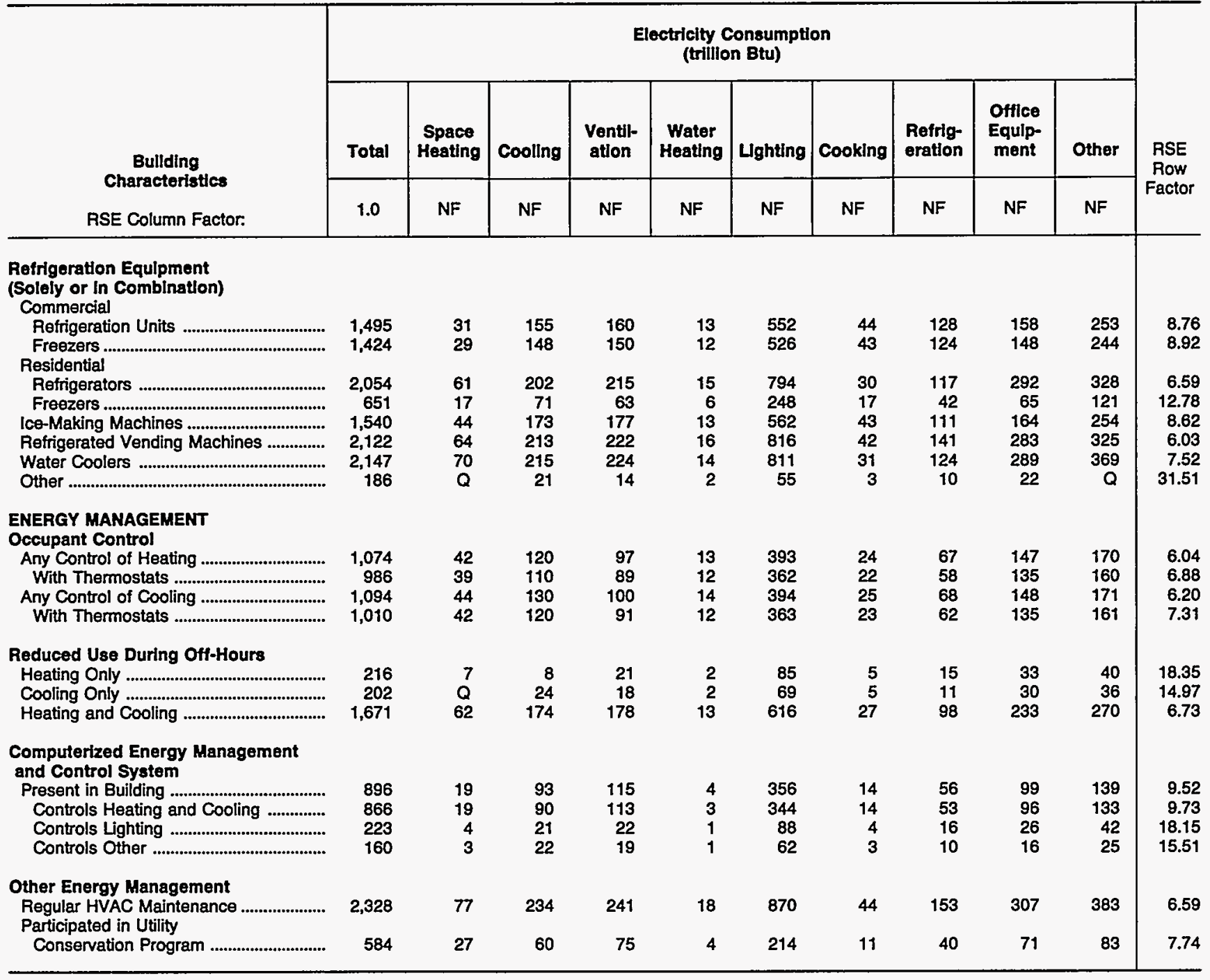

- = Value rounds to zero in the units displayed.

$\mathrm{NC}=$ No cases in responding sample.

NF $=$ No applicable RSE row/column factor.

$\mathrm{Q}=$ Data withheld because the Relative Standard Error (RSE) was greater than 50 percent, or data were reported for fewer than 20 buildings.

Notes: - To obtain the RSE percentage for any table cell, multiply the corresponding RSE column and RSE row factors. - See Glossary for explanation of abbreviations and definitions of terms used in this report. Because of rounding, data may not sum to totals.

Source: Energy Information Administration, Office of Energy Markets and End Use, Forms ElA-871A through F of the 1989 Commercial Buildings Energy Consumption Survey. 


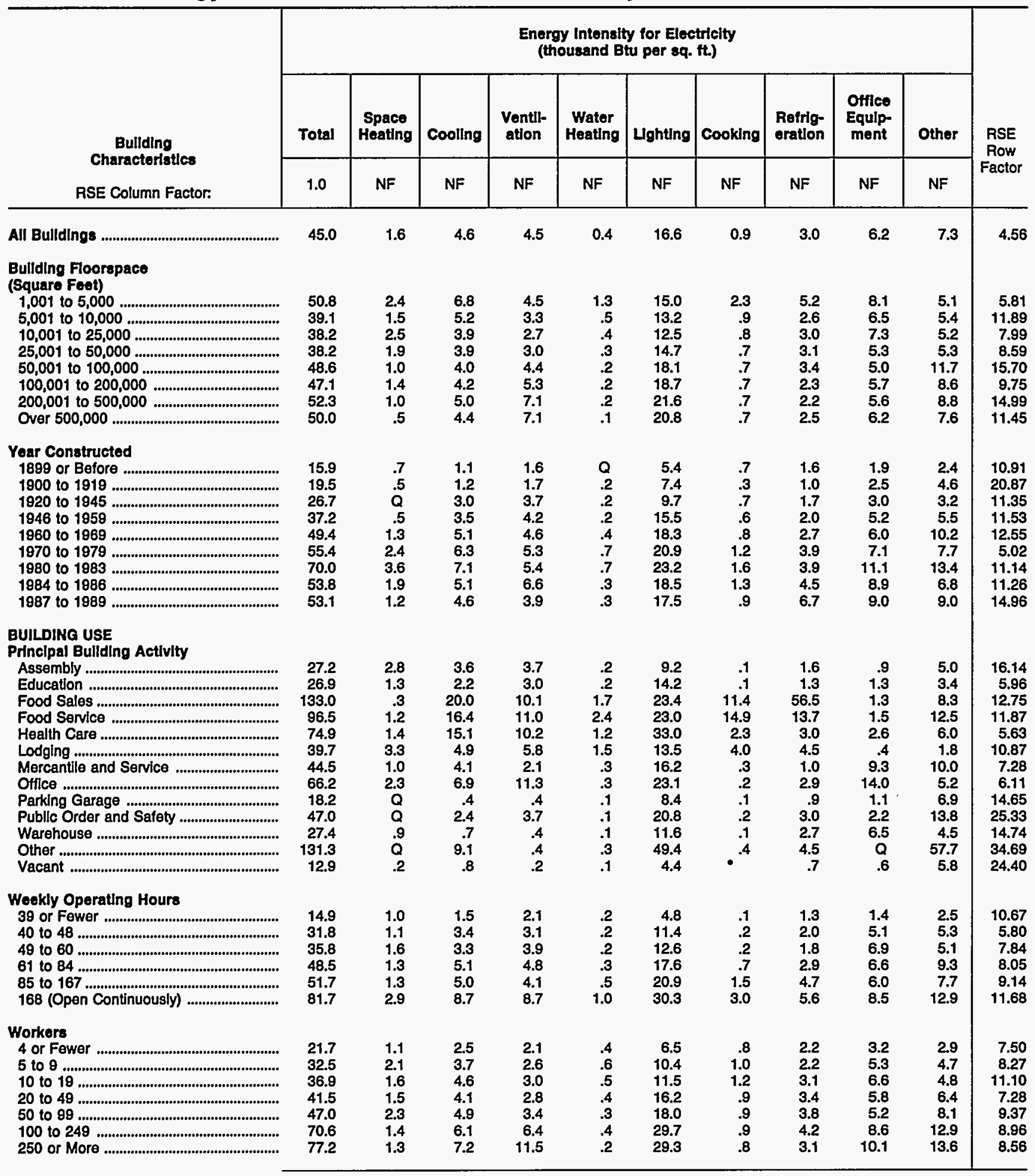

See footnote at end of table. 
Table B5. Energy End-Use Intensities for Electricity, 1989 (Continued)

\begin{tabular}{|c|c|c|c|c|c|c|c|c|c|c|c|}
\hline \multirow{3}{*}{$\begin{array}{c}\text { Bullding } \\
\text { Characteristics } \\
\text { RSE Column Factor: }\end{array}$} & \multicolumn{10}{|c|}{$\begin{array}{l}\text { Energy Intensity for Electricity } \\
\text { (thousand Btu per sq. } f t \text { ) }\end{array}$} & \multirow{3}{*}{$\begin{array}{c}\text { RSE } \\
\text { Row } \\
\text { Factor }\end{array}$} \\
\hline & Total & $\begin{array}{l}\text { Space } \\
\text { Heating }\end{array}$ & Coollng & $\begin{array}{l}\text { Ventil- } \\
\text { ation }\end{array}$ & $\begin{array}{l}\text { Water } \\
\text { Heatlng }\end{array}$ & Llghtlng & Cooklng & $\begin{array}{l}\text { Refrig- } \\
\text { eration }\end{array}$ & $\begin{array}{c}\text { Otflce } \\
\text { Equip- } \\
\text { ment }\end{array}$ & Other & \\
\hline & 1.0 & NF & NF & NF & NF & NF & NF & NF & NF & NF & \\
\hline \multicolumn{12}{|l|}{ Ownership and Occupancy } \\
\hline 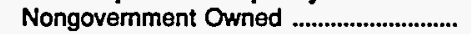 & 44.4 & 1.6 & 4.8 & 4.4 & 0.4 & 15.9 & 1.0 & 3.2 & 6.5 & 6.5 & 4.17 \\
\hline 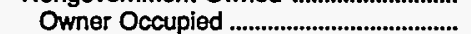 & 44.5 & 1.6 & 4.9 & 4.7 & .4 & 16.0 & 1.1 & 3.4 & 5.9 & 6.4 & 4.96 \\
\hline 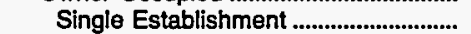 & 44.5 & 1.7 & 5.0 & 4.3 & .5 & 15.8 & 1.3 & 3.9 & 5.7 & 6.3 & 6.69 \\
\hline Multiple Establishment ........................... & 44.4 & 1.6 & 4.6 & 5.8 & .2 & 16.6 & .4 & 1.8 & 6.8 & 6.7 & 4.71 \\
\hline Nonowner Occupied ................................. & 44.4 & 1.4 & 4.5 & 3.6 & .4 & 15.8 & .8 & 2.7 & 8.3 & 6.8 & 7.03 \\
\hline Single Establishment ..................................... & 42.8 & 1.1 & 4.0 & 3.4 & .5 & 15.8 & 1.0 & 3.5 & 8.3 & 5.2 & 11.25 \\
\hline Multiple Establishment .......................... & 50.6 & 2.0 & 5.5 & 4.3 & .3 & 17.4 & .7 & 2.0 & 9.2 & 9.2 & 7.76 \\
\hline 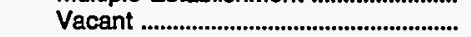 & 11.2 & .2 & 1.1 & .3 & .1 & 4.4 & 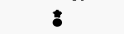 & .7 & .6 & 3.7 & 23.46 \\
\hline 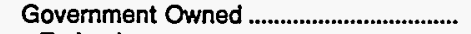 & 47.1 & 1.4 & 3.9 & 4.9 & .2 & 19.0 & .6 & 2.4 & 4.9 & 9.9 & 13.06 \\
\hline 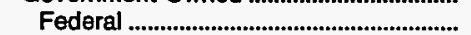 & 69.4 & 1.7 & 4.2 & 8.7 & .5 & 31.5 & .4 & 2.6 & 8.5 & 11.3 & $\$ 7.87$ \\
\hline 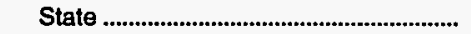 & 62.1 & 1.1 & 4.9 & 5.6 & .2 & 21.5 & 1.2 & 3.6 & 7.5 & $\mathbf{Q}$ & 27.97 \\
\hline 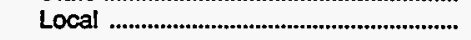 & 35.0 & 1.5 & 3.3 & 3.7 & .2 & 14.8 & .3 & 1.8 & 2.8 & 6.5 & 10.86 \\
\hline \multicolumn{12}{|l|}{ Multibuilding Facility } \\
\hline Not on Multibuilding Facility .......................... & 39.1 & 1.3 & 4.0 & 4.0 & .4 & 14.0 & 1.0 & 3.1 & 5.8 & 5.5 & 3.49 \\
\hline $\begin{array}{l}\text { Part of Multibuilding Facility ............................ } \\
\text { On Facility with Central }\end{array}$ & 53.7 & 2.0 & 5.4 & 5.3 & .3 & 20.5 & .8 & 2.9 & 6.6 & 10.0 & 8.23 \\
\hline 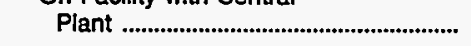 & 76.5 & 1.5 & 6.9 & 8.0 & .3 & 30.2 & 1.4 & 4.6 & 7.7 & 15.9 & 15.60 \\
\hline \multicolumn{12}{|l|}{$\begin{array}{l}\text { Percent Vacant at Least Three } \\
\text { Months }\end{array}$} \\
\hline 0 & 48.3 & 1.7 & 4.9 & 4.5 & .5 & 17.7 & 1.1 & 3.5 & 6.7 & 7.5 & 5.74 \\
\hline 1 to 50 & 47.0 & 1.3 & 5.0 & 5.7 & .2 & 17.5 & .5 & 2.0 & 6.4 & 8.3 & 4.88 \\
\hline 51 to 99 & 18.6 & $Q$ & 1.0 & 1.9 & .1 & 8.2 & $\mathrm{Q}$ & 1.3 & 1.8 & 3.4 & 24.80 \\
\hline 100 & 21.1 & 1.0 & 2.0 & 2.1 & .4 & 7.1 & .5 & 2.1 & 1.9 & 3.9 & 9.94 \\
\hline \multicolumn{12}{|l|}{ Months In Use Out of Past 12 Months } \\
\hline 0 to 8 & 24.4 & 1.0 & 2.3 & 2.1 & .3 & 7.7 & .7 & 2.4 & 2.9 & 5.0 & 15.78 \\
\hline 8 to 11 & 20.2 & 1.4 & 1.9 & 3.0 & .2 & 8.4 & .3 & 1.1 & 1.1 & 2.8 & 7.80 \\
\hline 12 & 48.0 & 1.6 & 4.9 & 4.8 & .4 & 17.7 & 1.0 & 3.2 & 6.7 & 7.7 & 4.88 \\
\hline \multicolumn{12}{|l|}{$\begin{array}{l}\text { LOCATION } \\
\text { Census Region }\end{array}$} \\
\hline 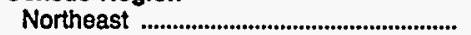 & 43.9 & 1.7 & 2.6 & 4.3 & .2 & 17.1 & .8 & 2.6 & 6.9 & 7.7 & 11.20 \\
\hline Midwest ............................................................... & 38.8 & 1.7 & 3.3 & 3.7 & .4 & 15.3 & .9 & 3.1 & 5.0 & 5.4 & 6.11 \\
\hline South & 45.9 & 1.5 & 6.4 & 4.4 & .6 & 16.1 & .9 & 3.1 & 5.8 & 7.3 & 4.32 \\
\hline 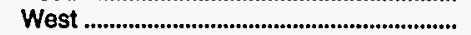 & 53.3 & 1.4 & 5.4 & 6.1 & 3 & 18.9 & 1.0 & 3.4 & 7.6 & 8.4 & 11.62 \\
\hline Census Division & & & & & & & & & & & \\
\hline & & & & & & & & & & & \\
\hline 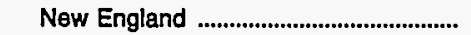 & 36.9 & 1.2 & 2.4 & 4.0 & .2 & 14.6 & .9 & 2.4 & 5.1 & 6.0 & 9.87 \\
\hline Middle Atlantic ...... & 46.1 & 1.9 & 2.7 & 4.3 & .2 & 17.8 & .8 & 2.7 & 7.4 & 8.2 & 13.83 \\
\hline Midwest & & & & & & & & & & & \\
\hline 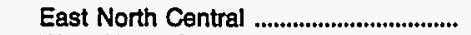 & 37.9 & 1.6 & 2.6 & 3.5 & .4 & 15.2 & .9 & 3.3 & 5.1 & 5.4 & 8.31 \\
\hline West North Central ................................ & 40.5 & 1.8 & 4.6 & 4.2 & .3 & 15.5 & 1.0 & 2.7 & 4.8 & 5.6 & 8.46 \\
\hline South & & & & & & & & & & & \\
\hline 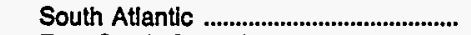 & 43.2 & 1.3 & 6.3 & 4.5 & .6 & 15.2 & .7 & 2.6 & 5.7 & 6.2 & 6.60 \\
\hline 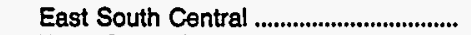 & 51.0 & 2.6 & 4.8 & 4.1 & .8 & 16.9 & 1.4 & 3.6 & 7.1 & 9.8 & 15.25 \\
\hline 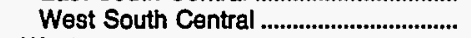 & 46.7 & 1.0 & 7.3 & 4.5 & .4 & 16.7 & 1.0 & 3.4 & 5.1 & 7.3 & 8.48 \\
\hline West & & & & & & & & & & & \\
\hline 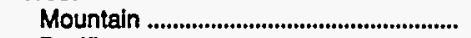 & 42.8 & 1.3 & 4.7 & 5.8 & .3 & 15.8 & .9 & 2.8 & 5.8 & 5.5 & 14.73 \\
\hline 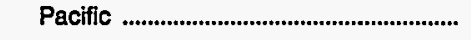 & 59.5 & 1.4 & 5.7 & 6.3 & .3 & 20.7 & 1.0 & 3.7 & 8.7 & 11.6 & 15.04 \\
\hline Itan Status & & & & & & & & & & & \\
\hline 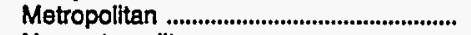 & 47.5 & 1.4 & 4.9 & 4.9 & .3 & 17.5 & .9 & 3.1 & 6.5 & 7.8 & 5.01 \\
\hline 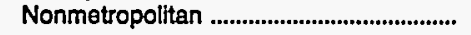 & 34.7 & 2.1 & 3.1 & 2.9 & .6 & 12.7 & 1.0 & 2.6 & 4.7 & 5.0 & 10.12 \\
\hline
\end{tabular}

See footnote at end of table. 
Table B5. Energy End-Use Intensities for Electricity, 1989 (Continued)

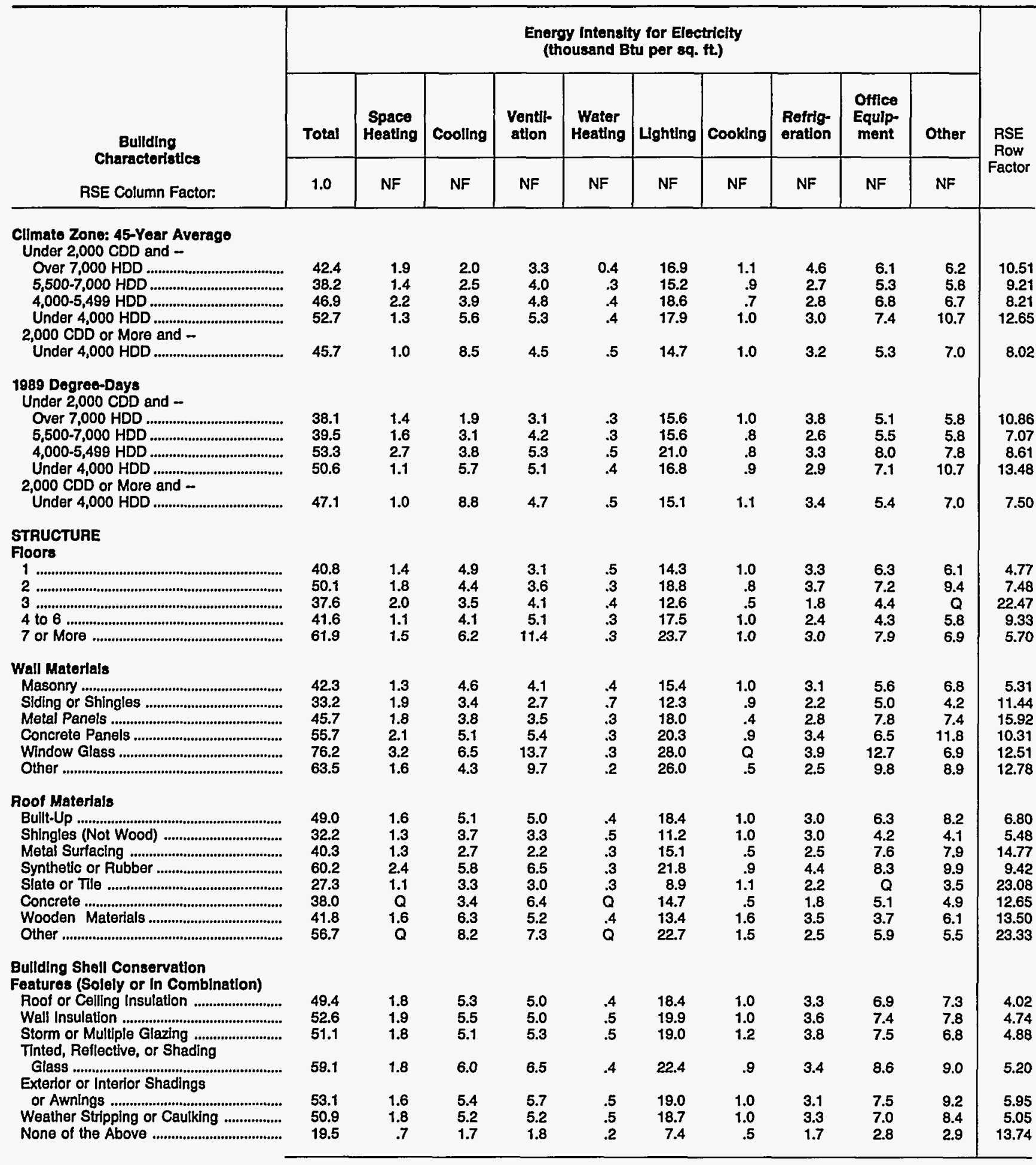

See footnote at end of table. 


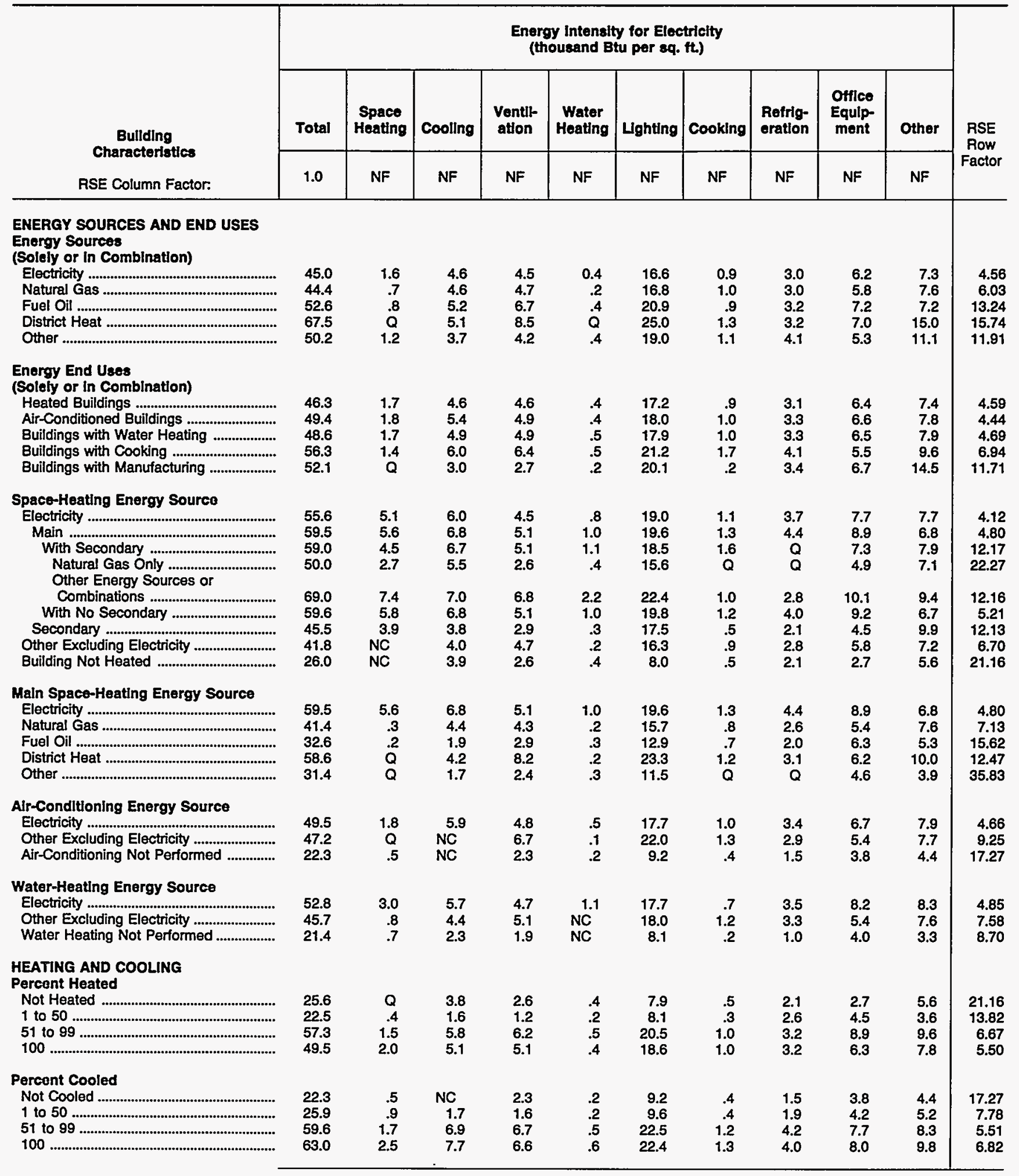

See tootnotes at end of table. 
Table B5. Energy End-Use Intensities for Electricity, 1989 (Continued)

\begin{tabular}{|c|c|c|c|c|c|c|c|c|c|c|c|}
\hline \multirow{3}{*}{$\begin{array}{c}\text { Bullding } \\
\text { Characteristics } \\
\text { RSE Column Factor: }\end{array}$} & \multicolumn{10}{|c|}{$\begin{array}{l}\text { Energy Intensity for Eloctriclty } \\
\text { (thousand Btu per sq. ft) }\end{array}$} & \multirow{3}{*}{$\begin{array}{l}\text { RSE } \\
\text { Row } \\
\text { Factor }\end{array}$} \\
\hline & \multirow{2}{*}{$\begin{array}{c}\text { Total } \\
1.0\end{array}$} & \multirow{2}{*}{$\begin{array}{c}\begin{array}{c}\text { Space } \\
\text { Heating }\end{array} \\
\text { NF }\end{array}$} & \multirow{2}{*}{\begin{tabular}{c|} 
Cooling \\
NF
\end{tabular}} & \multirow{2}{*}{$\begin{array}{c}\begin{array}{c}\text { Ventll- } \\
\text { ation }\end{array} \\
\mathrm{NF}\end{array}$} & \multirow{2}{*}{\begin{tabular}{|c|}
$\begin{array}{c}\text { Wator } \\
\text { Heating }\end{array}$ \\
NF \\
\end{tabular}} & \multirow{2}{*}{\begin{tabular}{|c|} 
Lighting \\
$\mathrm{NF}$
\end{tabular}} & \multirow{2}{*}{\begin{tabular}{|c|} 
Cooking \\
$\mathrm{NF}$
\end{tabular}} & \multirow{2}{*}{$\begin{array}{c}\begin{array}{c}\text { Refing- } \\
\text { eration }\end{array} \\
\text { NF }\end{array}$} & \multirow{2}{*}{$\begin{array}{c}\begin{array}{c}\text { Office } \\
\text { Equlp- } \\
\text { ment }\end{array} \\
\mathrm{NF}\end{array}$} & \multirow{2}{*}{$\frac{\text { Other }}{\text { NF }}$} & \\
\hline & & & & & & & & & & & \\
\hline \multicolumn{12}{|l|}{$\begin{array}{l}\text { Hoating Equipment } \\
\text { (Solely or In Combination) }\end{array}$} \\
\hline Furnaces ....................................... & 39.0 & 1.4 & 3.5 & 2.9 & 0.4 & 14.1 & 0.9 & 3.4 & 6.4 & 6.1 & 6.69 \\
\hline Bollers .............................................. & 44.0 & .9 & 4.3 & 5.5 & .2 & 17.8 & .7 & 2.8 & 5.2 & 6.6 & 7.21 \\
\hline Individual Space Heaters .... & 41.8 & 2.2 & 3.5 & 3.6 & .4 & 15.9 & .7 & 2.4 & 5.9 & 7.1 & 5.96 \\
\hline 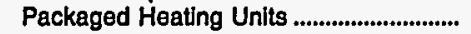 & 56.3 & 2.0 & 6.4 & 4.4 & .5 & 20.3 & 1.1 & 3.2 & 7.5 & 10.9 & 8.36 \\
\hline Heat Pumps ............................................................. & 51.0 & 3.1 & 5.9 & 5.1 & .5 & 17.7 & 1.0 & 3.7 & 6.9 & 7.2 & 7.31 \\
\hline Alr Ducts & 53.8 & 1.6 & 5.5 & 5.5 & .4 & 20.2 & 1.0 & 3.4 & 7.4 & 8.9 & 5.08 \\
\hline 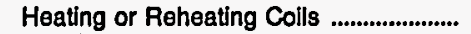 & 66.3 & 1.8 & 6.2 & 8.1 & .3 & 25.6 & 1.0 & 3.3 & 7.9 & 12.2 & 7.39 \\
\hline $\begin{array}{l}\text { Fan-Coil Units } \\
\text { Steam or Hot Water Radiators }\end{array}$ & 57.6 & $\mathbf{a}$ & 5.0 & 6.7 & .3 & 23.0 & .9 & 2.9 & 6.5 & 10.9 & 12.44 \\
\hline or Baseboards .................................................... & 38.2 & .8 & 2.9 & 4.9 & .2 & 16.2 & .7 & 2.2 & 4.5 & 5.9 & 8.42 \\
\hline Other & 79.5 & $\mathbf{Q}$ & 9.2 & 12.2 & .6 & 27.8 & 1.6 & 6.0 & 9.3 & 9.3 & 19.54 \\
\hline \multicolumn{12}{|l|}{$\begin{array}{l}\text { Cooling Equipment } \\
\text { (Sololy or In Combination) }\end{array}$} \\
\hline Central Chillers ............................ & 65.7 & 2.0 & 7.2 & 9.4 & .3 & 24.5 & .9 & 3.0 & 7.7 & 10.7 & 8.69 \\
\hline 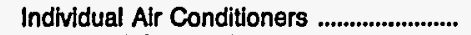 & 39.2 & 1.5 & 3.9 & 3.6 & .5 & 14.0 & .8 & 2.7 & 4.5 & 7.8 & 10.70 \\
\hline Packaged Cooling Units ................................ & 51.9 & 1.5 & 5.8 & 4.9 & .4 & 18.9 & 1.1 & 3.5 & 7.0 & 8.8 & 4.63 \\
\hline Heat Pumps ........................................................... & 59.7 & 3.3 & 6.1 & 5.1 & .6 & 19.4 & .9 & 3.9 & 8.1 & 12.2 & 16.07 \\
\hline 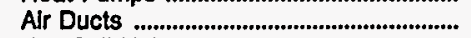 & 54.9 & 1.9 & 6.0 & 5.7 & .4 & 20.4 & 1.0 & 3.4 & 7.2 & 8.9 & 4.98 \\
\hline 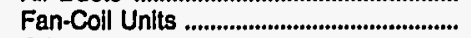 & 69.8 & 2.1 & 7.0 & 10.0 & .3 & 26.7 & 1.0 & 3.2 & 8.3 & 11.2 & 10.01 \\
\hline Other & 43.2 & .6 & 5.5 & 3.3 & .3 & 19.7 & .4 & 1.8 & 5.7 & 5.8 & 15.18 \\
\hline \multicolumn{12}{|l|}{ Year Main Central Chiller Installed } \\
\hline 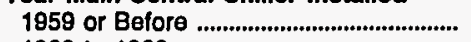 & 61.8 & $\mathbf{Q}$ & 7.1 & 10.8 & .3 & 21.7 & .8 & 2.5 & 7.0 & 5.6 & 17.17 \\
\hline 1960 to 1969 & 67.8 & .9 & 7.0 & 7.7 & .1 & 24.3 & 6 & 2.6 & 7.0 & $\mathbf{Q}$ & 29.29 \\
\hline 1970 to 1979 & 65.4 & 1.4 & 6.9 & 9.2 & .5 & 25.2 & .9 & 3.0 & 8.2 & 10.3 & 12.80 \\
\hline 1980 to 1986 & 69.6 & 2.6 & 7.9 & 11.8 & .3 & 26.5 & 1.1 & 3.5 & 8.7 & 7.4 & 11.59 \\
\hline 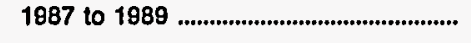 & 57.1 & 1.0 & 6.8 & 7.8 & $Q$ & 22.1 & 1.1 & 3.6 & 6.6 & 7.5 & 16.61 \\
\hline \multicolumn{12}{|l|}{$\begin{array}{l}\text { Year Packaged Cooling System } \\
\text { Installed }\end{array}$} \\
\hline 1859 or Before & 45.4 & .4 & 4.9 & 5.3 & .1 & 20.7 & .3 & 1.8 & 5.3 & 6.6 & 14.05 \\
\hline 1960 to $1969 \ldots$ & 57.0 & 1.1 & 5.2 & 4.4 & .3 & 20.3 & .7 & 2.6 & 7.5 & $\mathbf{Q}$ & 22.69 \\
\hline 1970 to 1979 & 52.3 & 2.2 & 6.4 & 5.0 & .5 & 19.5 & 1.2 & 3.4 & 6.9 & 7.0 & 6.37 \\
\hline 1980 to 1986 ....... & 52.9 & 1.7 & 5.7 & 4.9 & .4 & 18.8 & 1.2 & 4.2 & 7.8 & 8.2 & 8.89 \\
\hline 1987 to 1989 & 47.7 & .7 & 5.8 & 4.9 & .4 & 16.3 & 1.1 & 3.7 & 5.9 & 8.8 & 8.70 \\
\hline $\begin{array}{l}\text { Computer Area with Separate } \\
\text { Alr-Condltioning System }\end{array}$ & & & & & & & & & & & \\
\hline 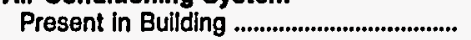 & 71.6 & 1.4 & 6.8 & 8.7 & .3 & 27.1 & .8 & 3.8 & 10.9 & 11.9 & 6.79 \\
\hline 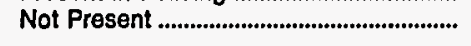 & $35 ?$ & 1.6 & 3.7 & 3.0 & .4 & 12.7 & 1.0 & 2.8 & 4.4 & 5.6 & 4.58 \\
\hline $\begin{array}{l}\text { LIGHTING AND REFRIGERATION } \\
\text { Percent LIt When Open }\end{array}$ & & & & & & & & & & & \\
\hline Not Lit & 10.5 & $\mathbf{Q}$ & .6 & .5 & .1 & 2.5 & .1 & .8 & 1.6 & 4.3 & 26.00 \\
\hline 1 to 50 & 17.7 & 1.3 & 1.8 & 2.0 & .3 & 4.4 & .4 & 1.4 & 2.9 & 3.2 & 9.47 \\
\hline 51 to 99 & 48.0 & 1.3 & 4.7 & 5.0 & .4 & 17.3 & .7 & 2.5 & 6.7 & 9.3 & 7.88 \\
\hline 100 & 53.3 & 1.8 & 5.5 & 5.2 & .4 & 20.6 & 1.2 & 3.9 & 7.0 & 7.7 & 4.72 \\
\hline Percent Llt When Closed & & & & & & & & & & & \\
\hline & 35.8 & 1.9 & 3.8 & 3.2 & .4 & 11.7 & .8 & 2.4 & 5.1 & 6.5 & 8.84 \\
\hline 1 to 50 & 49.2 & 1.3 & 4.7 & 5.0 & .4 & 19.3 & .8 & 3.2 & 6.9 & 7.7 & 4.53 \\
\hline 51 to 99 & 88.1 & $\mathbf{Q}$ & 10.1 & 11.5 & .7 & 35.0 & 2.5 & 5.7 & 9.7 & 12.0 & 18.57 \\
\hline 100 & 57.0 & .3 & 10.2 & 6.6 & Q & 20.0 & $\mathbf{Q}$ & $\mathbf{Q}$ & 1.8 & 5.2 & 29.63 \\
\hline $\begin{array}{l}\text { Lghting Equlpment } \\
\text { (Solely or in Comblnation) }\end{array}$ & & & & & & & & & & & \\
\hline 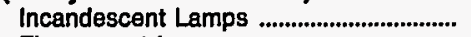 & 45.1 & 1.6 & 4.6 & 4.7 & .4 & 17.7 & 1.0 & 3.0 & 5.3 & 6.8 & 5.60 \\
\hline 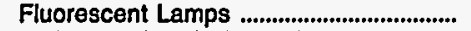 & 46.4 & 1.6 & 4.7 & 4.7 & .4 & 17.1 & .9 & 3.1 & 6.4 & 7.5 & 4.57 \\
\hline High-Intensity Discharge Lamps ............. & 54.0 & 1.7 & 4.7 & 5.2 & .2 & 21.8 & .7 & 3.5 & 6.7 & 9.5 & 7.76 \\
\hline 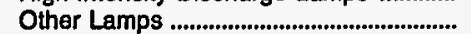 & 55.1 & $\mathbf{Q}$ & 7.2 & 7.0 & .6 & 22.7 & $\mathbf{Q}$ & $\mathbf{Q}$ & 5.2 & 5.9 & 15.06 \\
\hline 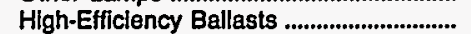 & 58.6 & 2.2 & 5.8 & 6.3 & .4 & 20.3 & 1.2 & 4.1 & 8.8 & 9.5 & 6.27 \\
\hline
\end{tabular}

See footnotes at end of table. 
Table B5. Energy End-Use Intensities for Electricity, 1989 (Continued)

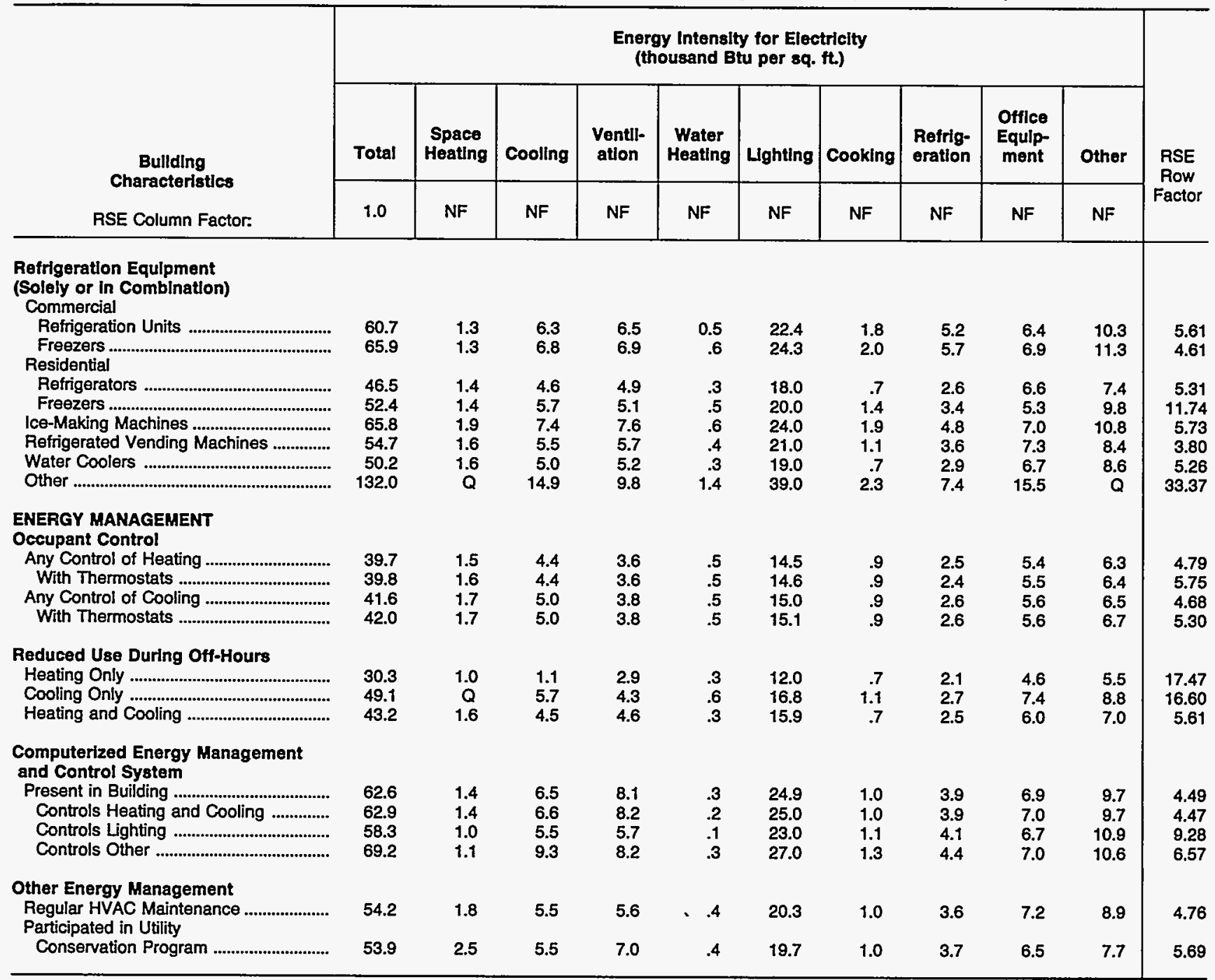

* = Value rounds to zero in the units displayed.

$\mathrm{NC}=$ No cases in responding sample.

NF $=$ No applicable RSE row/column factor.

$Q=$ Data withheld because the Relative Standard Error (RSE) was greater than 50 percent, or data were reported for fewer than 20 buildings.

Notes: - To obtain the RSE percentage for any table cell, multiply the corresponding RSE column and RSE row factors. - See Glossary for explanation of abbreviations and definitions of terms used in this report. - Because of rounding, data may not sum to totals.

Source: Energy Information Administration, Office of Energy Markets and End Use, Forms ElA-871A through F of the 1989 Commercial Buildings Energy Consumption Survey. 
Table B6. End-Use Consumption Percentages for Electricity, 1989

\begin{tabular}{|c|c|c|c|c|c|c|c|c|c|c|}
\hline \multirow[b]{2}{*}{$\begin{array}{c}\text { Bullding } \\
\text { Characterlstics }\end{array}$} & \multicolumn{10}{|c|}{ Percent of Electricity Consumption } \\
\hline & Total & $\begin{array}{c}\text { Space } \\
\text { Heating }\end{array}$ & Coollng & $\begin{array}{l}\text { Ventll- } \\
\text { ation }\end{array}$ & $\begin{array}{c}\text { Water } \\
\text { Heating }\end{array}$ & Llghting & Cooking & $\begin{array}{l}\text { Refrlg- } \\
\text { eration }\end{array}$ & $\begin{array}{c}\text { Office } \\
\text { Equilp- } \\
\text { ment }\end{array}$ & Other \\
\hline 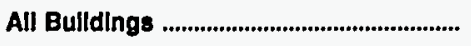 & 100 & 3 & 10 & 10 & 1 & 37 & 2 & 7 & 14 & 16 \\
\hline 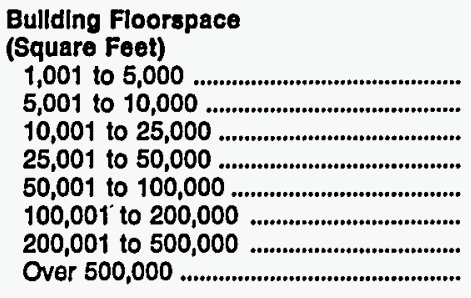 & $\begin{array}{l}100 \\
100 \\
100 \\
100 \\
100 \\
100 \\
100 \\
100\end{array}$ & $\begin{array}{l}5 \\
4 \\
6 \\
5 \\
2 \\
3 \\
2 \\
1\end{array}$ & $\begin{array}{r}13 \\
13 \\
10 \\
10 \\
8 \\
9 \\
10 \\
9\end{array}$ & $\begin{array}{r}9 \\
9 \\
7 \\
8 \\
9 \\
11 \\
14 \\
14\end{array}$ & $\begin{array}{r}3 \\
1 \\
1 \\
1 \\
+\quad 1 \\
0\end{array}$ & $\begin{array}{l}30 \\
34 \\
33 \\
38 \\
37 \\
40 \\
41 \\
42\end{array}$ & $\begin{array}{l}5 \\
2 \\
2 \\
2 \\
1 \\
1 \\
1 \\
1\end{array}$ & $\begin{array}{r}10 \\
7 \\
8 \\
8 \\
7 \\
5 \\
4 \\
5\end{array}$ & $\begin{array}{l}16 \\
17 \\
19 \\
14 \\
10 \\
12 \\
11 \\
12\end{array}$ & $\begin{array}{l}10 \\
14 \\
14 \\
14 \\
24 \\
18 \\
17 \\
15\end{array}$ \\
\hline 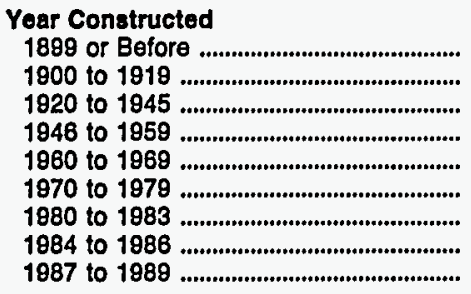 & $\begin{array}{l}100 \\
100 \\
100 \\
100 \\
100 \\
100 \\
100 \\
100 \\
100\end{array}$ & $\begin{array}{l}4 \\
3 \\
Q \\
1 \\
3 \\
4 \\
5 \\
4 \\
2\end{array}$ & $\begin{array}{r}7 \\
6 \\
11 \\
9 \\
10 \\
11 \\
10 \\
9 \\
9\end{array}$ & $\begin{array}{r}10 \\
9 \\
14 \\
11 \\
9 \\
10 \\
8 \\
12 \\
7\end{array}$ & $\begin{array}{l}3 \\
1 \\
1 \\
1 \\
1 \\
1 \\
1 \\
1 \\
1\end{array}$ & $\begin{array}{l}34 \\
38 \\
36 \\
42 \\
37 \\
38 \\
33 \\
34 \\
33\end{array}$ & $\begin{array}{l}4 \\
1 \\
3 \\
1 \\
2 \\
2 \\
2 \\
2 \\
2\end{array}$ & $\begin{array}{r}10 \\
5 \\
6 \\
5 \\
5 \\
7 \\
6 \\
8 \\
13\end{array}$ & $\begin{array}{l}12 \\
13 \\
11 \\
14 \\
12 \\
13 \\
16 \\
17 \\
17\end{array}$ & $\begin{array}{l}15 \\
24 \\
12 \\
15 \\
21 \\
14 \\
19 \\
13 \\
17\end{array}$ \\
\hline 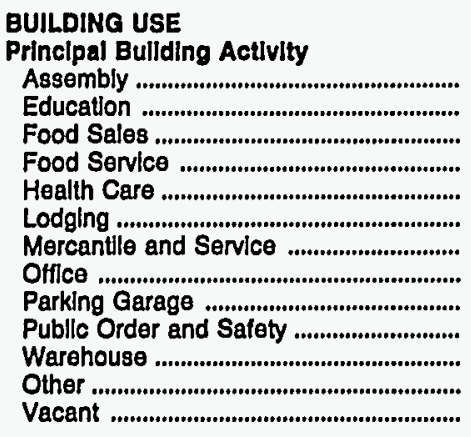 & $\begin{array}{l}100 \\
100 \\
100 \\
100 \\
100 \\
100 \\
100 \\
100 \\
100 \\
100 \\
100 \\
100 \\
100\end{array}$ & $\begin{array}{r}10 \\
5 \\
. \\
1 \\
2 \\
8 \\
2 \\
3 \\
Q \\
Q \\
3 \\
Q \\
2\end{array}$ & $\begin{array}{r}13 \\
8 \\
15 \\
17 \\
20 \\
12 \\
9 \\
10 \\
2 \\
5 \\
2 \\
7 \\
6\end{array}$ & $\begin{array}{r}14 \\
11 \\
8 \\
11 \\
14 \\
15 \\
5 \\
17 \\
2 \\
8 \\
1 \\
1 \\
1\end{array}$ & $\begin{array}{l}1 \\
1 \\
1 \\
2 \\
2 \\
4 \\
1 \\
0 \\
\vdots \\
\vdots \\
\vdots\end{array}$ & $\begin{array}{l}34 \\
53 \\
18 \\
24 \\
44 \\
34 \\
37 \\
35 \\
46 \\
44 \\
42 \\
38 \\
34\end{array}$ & $\begin{array}{r}: \\
9 \\
15 \\
3 \\
10 \\
1 \\
: \\
: \\
: 1\end{array}$ & $\begin{array}{r}6 \\
5 \\
42 \\
14 \\
4 \\
11 \\
2 \\
4 \\
5 \\
6 \\
10 \\
3 \\
6\end{array}$ & $\begin{array}{r}3 \\
5 \\
1 \\
2 \\
4 \\
1 \\
21 \\
21 \\
6 \\
5 \\
24 \\
6 \\
5\end{array}$ & $\begin{array}{r}19 \\
13 \\
6 \\
13 \\
8 \\
5 \\
23 \\
8 \\
38 \\
29 \\
16 \\
44 \\
45\end{array}$ \\
\hline 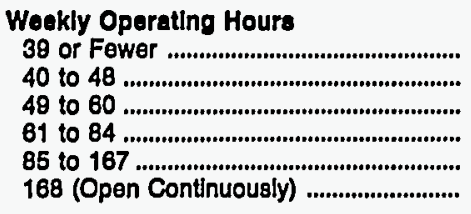 & $\begin{array}{l}100 \\
100 \\
100 \\
100 \\
100 \\
100\end{array}$ & $\begin{array}{l}7 \\
4 \\
5 \\
3 \\
3 \\
4\end{array}$ & $\begin{array}{r}10 \\
11 \\
9 \\
10 \\
10 \\
11\end{array}$ & $\begin{array}{r}14 \\
10 \\
11 \\
10 \\
8 \\
11\end{array}$ & $\begin{array}{l}2 \\
1 \\
1 \\
1 \\
1 \\
1\end{array}$ & $\begin{array}{l}32 \\
36 \\
35 \\
36 \\
40 \\
37\end{array}$ & $\begin{array}{l}1 \\
1 \\
1 \\
1 \\
3 \\
4\end{array}$ & $\begin{array}{l}9 \\
6 \\
5 \\
6 \\
9 \\
7\end{array}$ & $\begin{array}{r}9 \\
16 \\
19 \\
14 \\
12 \\
10\end{array}$ & $\begin{array}{l}17 \\
17 \\
14 \\
19 \\
15 \\
16\end{array}$ \\
\hline 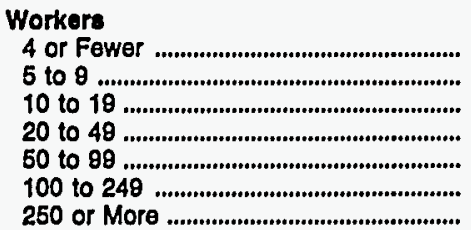 & $\begin{array}{l}100 \\
100 \\
100 \\
100 \\
100 \\
100 \\
100\end{array}$ & $\begin{array}{l}5 \\
6 \\
4 \\
4 \\
5 \\
2 \\
2\end{array}$ & $\begin{array}{r}11 \\
11 \\
13 \\
10 \\
10 \\
9 \\
9\end{array}$ & $\begin{array}{r}10 \\
8 \\
8 \\
7 \\
7 \\
9 \\
15\end{array}$ & $\begin{array}{r}2 \\
2 \\
1 \\
1 \\
1 \\
1 \\
.\end{array}$ & $\begin{array}{l}30 \\
32 \\
31 \\
39 \\
38 \\
42 \\
38\end{array}$ & $\begin{array}{l}4 \\
3 \\
3 \\
2 \\
2 \\
1 \\
1\end{array}$ & $\begin{array}{r}10 \\
7 \\
8 \\
8 \\
8 \\
6 \\
4\end{array}$ & $\begin{array}{l}15 \\
16 \\
18 \\
14 \\
11 \\
12 \\
13\end{array}$ & $\begin{array}{l}13 \\
14 \\
13 \\
15 \\
17 \\
18 \\
18\end{array}$ \\
\hline
\end{tabular}

See footnotes at end of table. 
Table B6. End-Use Consumption Percentages for Electricity, 1989 (Continued)

\begin{tabular}{|c|c|c|c|c|c|c|c|c|c|c|}
\hline \multirow[b]{2}{*}{$\begin{array}{l}\text { Building } \\
\text { Characteristlcs }\end{array}$} & \multicolumn{10}{|c|}{ Percent of Electricity Consumption } \\
\hline & Total & $\begin{array}{l}\text { Space } \\
\text { Heating }\end{array}$ & Cooling & $\begin{array}{l}\text { Ventl- } \\
\text { atton }\end{array}$ & $\begin{array}{l}\text { Water } \\
\text { Heating }\end{array}$ & Lghting & Cooklng & $\begin{array}{l}\text { Refrig- } \\
\text { eration }\end{array}$ & $\begin{array}{l}\text { Ottice } \\
\text { Equip- } \\
\text { ment }\end{array}$ & Other \\
\hline \multicolumn{11}{|l|}{ Ownershlp and Occupancy } \\
\hline 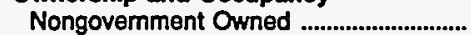 & 100 & 4 & 11 & 10 & 1 & 36 & 2 & 7 & 15 & 15 \\
\hline 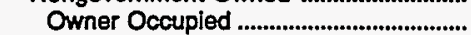 & 100 & 4 & 11 & 11 & $i$ & 36 & 2 & 8 & 13 & 14 \\
\hline 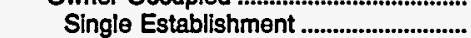 & 100 & 4 & 11 & 10 & $i$ & 35 & 3 & 9 & 13 & 14 \\
\hline 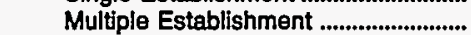 & 100 & 4 & 10 & 13 & 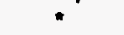 & 37 & 1 & 4 & 15 & 15 \\
\hline 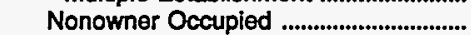 & 100 & 3 & 10 & 8 & 1 & 36 & 2 & 6 & 19 & 15 \\
\hline Single Establishment .............................. & 100 & 3 & 9 & 8 & 1 & 37 & 2 & 8 & 19 & 12 \\
\hline 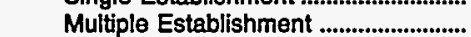 & 100 & 4 & 11 & 8 & $i$ & 34 & 1 & 4 & 18 & 18 \\
\hline 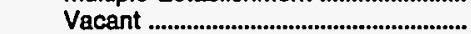 & 100 & 2 & 10 & 2 & 1 & 39 & $*$ & 6 & 6 & 33 \\
\hline 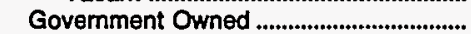 & 100 & 3 & 8 & 10 & $i$ & 40 & 1 & 5 & 10 & 21 \\
\hline 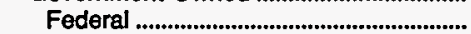 & 100 & $\mathbf{Q}$ & 6 & 13 & $\dot{Q}$ & 45 & 1 & 4 & 12 & 16 \\
\hline 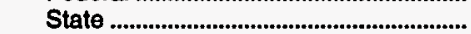 & 100 & 2 & 8 & 9 & 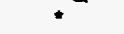 & 35 & 2 & 6 & 12 & 26 \\
\hline 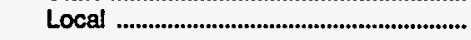 & 100 & 4 & 9 & 11 & 1 & 42 & 1 & 5 & 8 & 19 \\
\hline \multicolumn{11}{|l|}{ Multibuilding Facility } \\
\hline Not on Multibuilding Facility ......................... & 100 & 3 & 10 & 10 & 1 & 36 & 3 & 8 & 15 & 14 \\
\hline $\begin{array}{l}\text { Part of Multibuilding Facility .............................. } \\
\text { On Facility with Contral }\end{array}$ & 100 & 4 & 10 & 10 & 1 & 38 & 1 & 5 & 12 & 19 \\
\hline 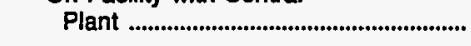 & 100 & 2 & 9 & 11 & * & 39 & 2 & 6 & 10 & 21 \\
\hline \multicolumn{11}{|l|}{$\begin{array}{l}\text { Percent Vacant at Least Three } \\
\text { Months }\end{array}$} \\
\hline 0 & 100 & 4 & 10 & 9 & 1 & 37 & 2 & 7 & 14 & 16 \\
\hline 1 to 50 & 100 & 3 & 11 & 12 & 1 & 37 & 1 & 4 & 14 & 18 \\
\hline 51 to 99 & 100 & 3 & 5 & 10 & - & 44 & $Q$ & 7 & 10 & 18 \\
\hline 100 & 100 & 5 & 10 & 10 & 2 & 34 & 3 & 10 & 9 & 19 \\
\hline \multicolumn{11}{|l|}{ Months in Use Out of Past 12 Months } \\
\hline 0 to 8 & 100 & 4 & 10 & 9 & 1 & 31 & 3 & 10 & 12 & 20 \\
\hline 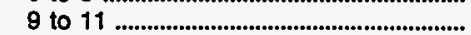 & 100 & 7 & 9 & 15 & $i$ & 42 & 1 & 6 & 5 & 14 \\
\hline 12 & 100 & 3 & 10 & 10 & 1 & 37 & 2 & 7 & 14 & 16 \\
\hline \multicolumn{11}{|l|}{$\begin{array}{l}\text { LOCATION } \\
\text { Census Region }\end{array}$} \\
\hline 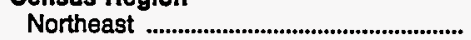 & 100 & 4 & 6 & 10 & 1 & 39 & 2 & 6 & 16 & 17 \\
\hline 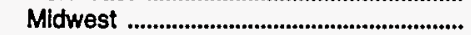 & 100 & 4 & 8 & 10 & 1 & 39 & 2 & 8 & 13 & 14 \\
\hline South & 100 & 3 & 14 & 10 & 1 & 35 & 2 & 7 & 13 & 16 \\
\hline West & 100 & 3 & 10 & 11 & 1 & 35 & 2 & 6 & 14 & 18 \\
\hline \multirow{2}{*}{\multicolumn{11}{|c|}{ Census Division }} \\
\hline \multicolumn{10}{|l|}{$\begin{array}{l}\text { Census Division } \\
\text { Northeast }\end{array}$} & \\
\hline 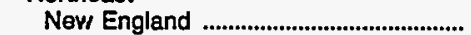 & 100 & 3 & 6 & 11 & 1 & 40 & 2 & 7 & 14 & 16 \\
\hline \multicolumn{11}{|l|}{ Midwest } \\
\hline 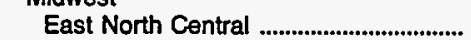 & 100 & 4 & 7 & 9 & 1 & 40 & 2 & 9 & 13 & 14 \\
\hline 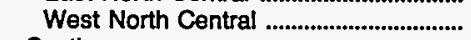 & 100 & 5 & 11 & 10 & $i$ & 38 & 2 & 7 & 12 & 14 \\
\hline \multicolumn{11}{|l|}{ South } \\
\hline 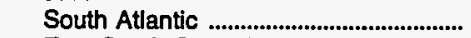 & 100 & 3 & 15 & 10 & 1 & 35 & 2 & 6 & 13 & 14 \\
\hline 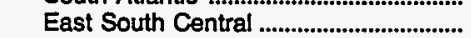 & 100 & 5 & 9 & 8 & 2 & 33 & 3 & 7 & 14 & 10 \\
\hline 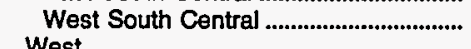 & 100 & 2 & 16 & 10 & 1 & 36 & 2 & 7 & 11 & 16 \\
\hline \multicolumn{11}{|l|}{ West } \\
\hline 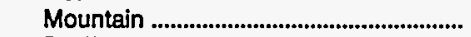 & 100 & 3 & 11 & 13 & 1 & 37 & 2 & 7 & 14 & 13 \\
\hline 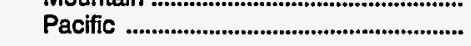 & 100 & 2 & 10 & 11 & $i$ & 35 & 2 & 6 & $\begin{array}{l}14 \\
15\end{array}$ & 20 \\
\hline \multicolumn{11}{|l|}{ Metropolltan Status } \\
\hline 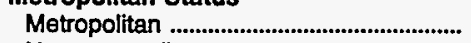 & 100 & 3 & 10 & 10 & 1 & 37 & 2 & 7 & 14 & 17 \\
\hline 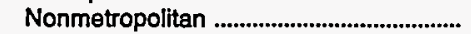 & 100 & 6 & 9 & 8 & 2 & 37 & 3 & 8 & 14 & 14 \\
\hline
\end{tabular}

See footnotes at end of table. 
Table B6. End-Use Consumption Percentages for Electricity, 1989 (Continued)

\begin{tabular}{|c|c|c|c|c|c|c|c|c|c|c|}
\hline \multirow[b]{2}{*}{$\begin{array}{l}\text { Bullding } \\
\text { Characterlatics }\end{array}$} & \multicolumn{10}{|c|}{ Percent of Electricity Consumption } \\
\hline & Total & $\begin{array}{l}\text { Space } \\
\text { Heating }\end{array}$ & Cooling & $\begin{array}{l}\text { Ventir } \\
\text { ation }\end{array}$ & $\begin{array}{c}\text { Water } \\
\text { Heating }\end{array}$ & Llghting & Cooklng & $\begin{array}{l}\text { Refrig- } \\
\text { eration }\end{array}$ & $\begin{array}{c}\text { Otflce } \\
\text { Equip- } \\
\text { ment }\end{array}$ & Other \\
\hline \multicolumn{11}{|l|}{$\begin{array}{l}\text { Cllmate Zone: 45-Year Averago } \\
\text { Under 2,000 CDD and -- }\end{array}$} \\
\hline Over 7,000 HDD & 100 & 5 & 5 & 8 & 1 & 40 & 3 & 11 & 14 & 15 \\
\hline $5,500-7,000 \mathrm{HDD}$ & 100 & 4 & 7 & 11 & 1 & 40 & 2 & 7 & 14 & 15 \\
\hline 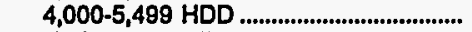 & 100 & 5 & 8 & 10 & 1 & 40 & 2 & 6 & 14 & 14 \\
\hline $\begin{array}{l}\text { Under 4,000 HDD } \\
2,000 \text { CDD or More and - }\end{array}$ & 100 & 2 & 11 & 10 & 1 & 34 & 2 & 6 & 14 & 20 \\
\hline 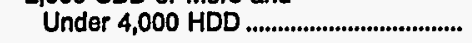 & 100 & 2 & 19 & 10 & 1 & 32 & 2 & 7 & 12 & 15 \\
\hline \multicolumn{11}{|l|}{$\begin{array}{l}1989 \text { Degree-Days } \\
\text { Under } 2,000 \text { CDD and - }\end{array}$} \\
\hline Over 7,000 HDD & $\begin{array}{l}100 \\
100\end{array}$ & $\begin{array}{l}4 \\
4\end{array}$ & $\begin{array}{l}5 \\
8\end{array}$ & $\begin{array}{r}8 \\
11\end{array}$ & $\begin{array}{l}1 \\
1\end{array}$ & $\begin{array}{l}41 \\
39\end{array}$ & $\begin{array}{l}3 \\
2\end{array}$ & $\begin{array}{r}10 \\
6\end{array}$ & $\begin{array}{l}13 \\
14\end{array}$ & $\begin{array}{l}15 \\
15\end{array}$ \\
\hline 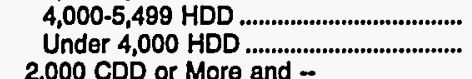 & $\begin{array}{l}100 \\
100\end{array}$ & $\begin{array}{l}5 \\
2\end{array}$ & $\begin{array}{r}7 \\
11\end{array}$ & $\begin{array}{l}10 \\
10\end{array}$ & $\begin{array}{l}1 \\
1\end{array}$ & $\begin{array}{l}39 \\
33\end{array}$ & $\begin{array}{l}2 \\
2\end{array}$ & $\begin{array}{l}6 \\
6\end{array}$ & $\begin{array}{l}15 \\
14\end{array}$ & $\begin{array}{l}15 \\
21\end{array}$ \\
\hline 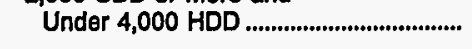 & 100 & 2 & 19 & 10 & 1 & 32 & 2 & 7 & 12 & 15 \\
\hline \multicolumn{11}{|l|}{ STRUCTURE } \\
\hline 1 & 100 & 3 & 12 & 8 & 1 & 35 & 3 & 8 & 15 & 15 \\
\hline 2 & 100 & 4 & 9 & 7 & 1 & 38 & 2 & 7 & 14 & 19 \\
\hline 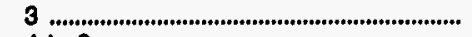 & 100 & 5 & 9 & 11 & 1 & 33 & 1 & 5 & 12 & 22 \\
\hline 4 to 6 & 100 & 3 & 10 & 12 & 1 & 42 & 2 & 6 & 10 & 14 \\
\hline 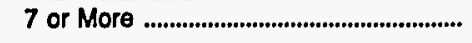 & 100 & 2 & 10 & 18 & 1 & 38 & 2 & 5 & 13 & 11 \\
\hline \multicolumn{11}{|l|}{ Wall Materlals } \\
\hline Masonry ........... & 100 & 3 & 11 & 10 & 1 & 36 & 2 & 7 & 13 & 16 \\
\hline 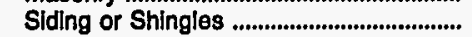 & 100 & 6 & 10 & 8 & 2 & 37 & 3 & 7 & 15 & 13 \\
\hline Metal Panels ................................................ & 100 & 4 & 8 & 8 & 1 & 39 & 1 & 6 & 17 & 16 \\
\hline 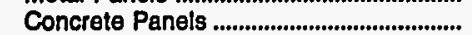 & 100 & 4 & 9 & 10 & 1 & 36 & 2 & 6 & 12 & 21 \\
\hline Window Glass ........................................................... & 100 & 4 & $\boldsymbol{9}$ & 18 & - & 37 & $Q$ & 5 & 17 & $\mathbf{9}$ \\
\hline 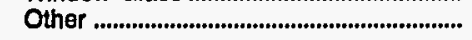 & 100 & 3 & 7 & 15 & - & 41 & $\overline{1}$ & 4 & 15 & 14 \\
\hline \multicolumn{11}{|l|}{ Roof Materials } \\
\hline Built-Up ............... & 100 & 3 & 10 & 10 & 1 & 38 & 2 & 6 & 13 & 17 \\
\hline 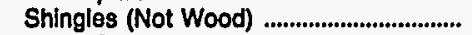 & 100 & 4 & 12 & 10 & 2 & 35 & 3 & 9 & 13 & 13 \\
\hline Metal Surfacing ........................................................ & 100 & 3 & 7 & 6 & 1 & 38 & 1 & 6 & 19 & 20 \\
\hline 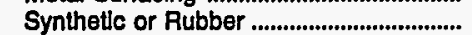 & 100 & 4 & 10 & 11 & 1 & 36 & 1 & 7 & 14 & 16 \\
\hline 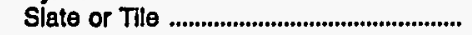 & 100 & 4 & 12 & 11 & 1 & 33 & 4 & 8 & 14 & 13 \\
\hline 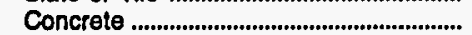 & 100 & $\dot{Q}$ & 9 & 17 & $\mathbf{Q}$ & 39 & $i$ & 5 & 13 & 13 \\
\hline 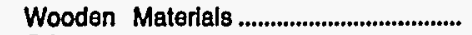 & 100 & 4 & 15 & 12 & 1 & 32 & 4 & 8 & 9 & 15 \\
\hline 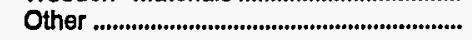 & 100 & $\mathbf{Q}$ & 14 & 13 & $\mathbf{Q}$ & 40 & 3 & 4 & 10 & 10 \\
\hline \multicolumn{11}{|l|}{$\begin{array}{l}\text { Bullding Shell Conservation } \\
\text { Features (Solely or In Combination) }\end{array}$} \\
\hline Roof or Ceilling Insulation ......................... & 100 & 4 & 11 & 10 & 1 & 37 & 2 & 7 & 14 & 15 \\
\hline Wall Insulation ................................................ & 100 & 4 & 10 & 10 & $i$ & 38 & 2 & 7 & 14 & 15 \\
\hline Storm or Multiple Glazing ............................. & 100 & 4 & 10 & 10 & 1 & 37 & 2 & 8 & 15 & 13 \\
\hline Tinted, Reflectlve, or Shading & & & & & & & & & & \\
\hline 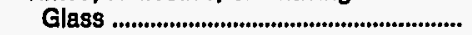 & 100 & 3 & 10 & 11 & 1 & 38 & 2 & 6 & 15 & 15 \\
\hline \multicolumn{11}{|l|}{ Exterior or Interior Shadings } \\
\hline 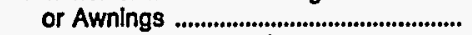 & 100 & 3 & 10 & 11 & 1 & 36 & 2 & 6 & 14 & 17 \\
\hline 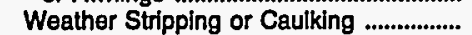 & 100 & 4 & 10 & 10 & 1 & 37 & 2 & 6 & 14 & 16 \\
\hline 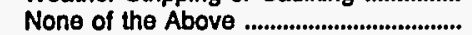 & 100 & 3 & 9 & $\mathbf{9}$ & 1 & 38 & 2 & $\mathbf{9}$ & 14 & 15 \\
\hline
\end{tabular}

See footnotes at end of table. 
Table B6. End-Use Consumption Percentages for Electricity, 1989 (Continued)

\begin{tabular}{|c|c|c|c|c|c|c|c|c|c|c|}
\hline \multirow[b]{2}{*}{$\begin{array}{c}\text { Bullding } \\
\text { Characteristics }\end{array}$} & \multicolumn{10}{|c|}{ Percent of Electricity Consumption } \\
\hline & Total & $\begin{array}{l}\text { Space } \\
\text { Heating }\end{array}$ & Coollng & $\begin{array}{l}\text { Ventil- } \\
\text { ation }\end{array}$ & $\begin{array}{l}\text { Water } \\
\text { Heating }\end{array}$ & Llghting & Cooking & $\begin{array}{l}\text { Refrig- } \\
\text { eration }\end{array}$ & $\begin{array}{l}\text { Otfice } \\
\text { Equip- } \\
\text { ment }\end{array}$ & Other \\
\hline \multicolumn{11}{|l|}{$\begin{array}{l}\text { ENERGY SOURCES AND END USES } \\
\text { Energy Sources } \\
\text { (Solely or In Combination) }\end{array}$} \\
\hline 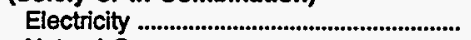 & 100 & 3 & 10 & 10 & 1 & 37 & 2 & 7 & 14 & 16 \\
\hline 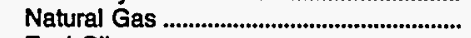 & 100 & 2 & 10 & 11 & 1 & 38 & 2 & 7 & 13 & 17 \\
\hline 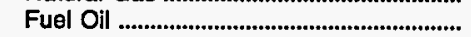 & 100 & 2 & 10 & 13 & $i$ & 40 & 2 & 6 & 14 & 14 \\
\hline 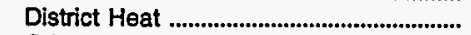 & 100 & $\vec{Q}$ & 8 & 13 & *' & 37 & 2 & 5 & 10 & 22 \\
\hline Other & 100 & 2 & 7 & 8 & 1 & 38 & 2 & 8 & 10 & 22 \\
\hline \multicolumn{11}{|l|}{$\begin{array}{l}\text { Energy End Usos } \\
\text { (Solely or in Combination) }\end{array}$} \\
\hline 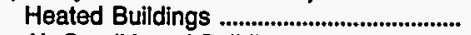 & 100 & 4 & 10 & 10 & 1 & 37 & 2 & 7 & 14 & 16 \\
\hline 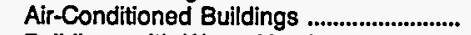 & 100 & 4 & 11 & 10 & 1 & 37 & 2 & 7 & 13 & 16 \\
\hline Buildings with Water Heating ........................ & 100 & 3 & 10 & 10 & 1 & 37 & 2 & 7 & 13 & 16 \\
\hline 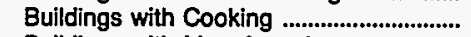 & 100 & 2 & 11 & 11 & 1 & 38 & 3 & 7 & 10 & 17 \\
\hline Buildings with Manufacturing ..................... & 100 & 3 & 6 & 5 & $*$ & 38 & * & 7 & 13 & 28 \\
\hline \multicolumn{11}{|l|}{ Space-Heating Energy Source } \\
\hline 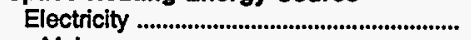 & 100 & 9 & 11 & 8 & 1 & 34 & 2 & 7 & 14 & 14 \\
\hline 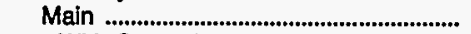 & 100 & $\boldsymbol{9}$ & 11 & 9 & 2 & 33 & 2 & 7 & 15 & 12 \\
\hline 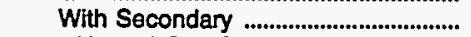 & 100 & 8 & 11 & 9 & 2 & 31 & 3 & 11 & 12 & 13 \\
\hline Natural Gas Only .................................. & 100 & 5 & 11 & 5 & 1 & 31 & $\mathbf{Q}$ & 18 & 10 & 14 \\
\hline Combinations ..................................... & 100 & 11 & 10 & 10 & 3 & 32 & 2 & 4 & 15 & 14 \\
\hline 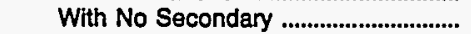 & 100 & 10 & 11 & 8 & 2 & 33 & 2 & 7 & 15 & 11 \\
\hline 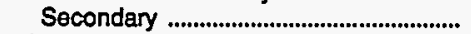 & 100 & 9 & 8 & 6 & 1 & 39 & 1 & 5 & 10 & 22 \\
\hline 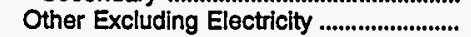 & 100 & NC & 9 & 11 & * & 39 & 2 & 7 & 14 & $\overline{17}$ \\
\hline 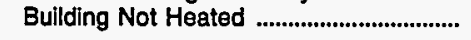 & 100 & NC & 15 & 10 & 2 & 31 & 2 & 8 & 10 & 22 \\
\hline \multicolumn{11}{|l|}{ Main Spaco-Heating Energy Source } \\
\hline 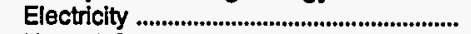 & 100 & 9 & 11 & 9 & 2 & 33 & 2 & 7 & 15 & 12 \\
\hline 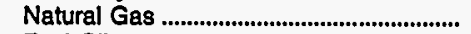 & 100 & 1 & 11 & 10 & - & 38 & 2 & 6 & 13 & 18 \\
\hline 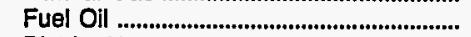 & 100 & 1 & 6 & 9 & 1 & 40 & 2 & 6 & 19 & 16 \\
\hline 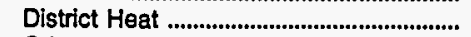 & 100 & $\dot{Q}$ & 7 & 14 & * & 40 & 2 & 5 & 11 & 17 \\
\hline Other & 100 & $\hat{Q}$ & 5 & 8 & $\cdot 1$ & 36 & $\mathbf{Q}$ & 19 & 15 & 13 \\
\hline \multicolumn{11}{|l|}{ Alr-Conditloning Energy Source } \\
\hline 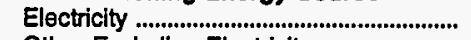 & 100 & 4 & 12 & 10 & 1 & 36 & 2 & 7 & 14 & 16 \\
\hline 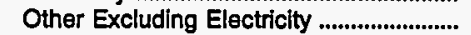 & 100 & 2 & NC & 14 & •" & 47 & 3 & 6 & 11 & 16 \\
\hline Alr-Conditioning Not Performed ................ & 100 & 2 & NC & 10 & 1 & 41 & 2 & 7 & 17 & 20 \\
\hline \multicolumn{11}{|l|}{ Water-Heating Energy Source } \\
\hline Electricity ........................................ & 100 & 6 & 11 & $\boldsymbol{\theta}$ & 2 & 34 & 1 & 7 & 15 & 16 \\
\hline 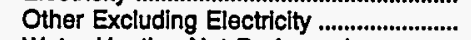 & 100 & 2 & 10 & 11 & NC & 39 & 3 & 7 & 12 & 17 \\
\hline Water Heating Not Performed .................... & 100 & 3 & 11 & $\theta$ & NC & 38 & 1 & 5 & 19 & 15 \\
\hline \multicolumn{11}{|l|}{$\begin{array}{l}\text { MEATING AND COOLING } \\
\text { Porcent Heated }\end{array}$} \\
\hline 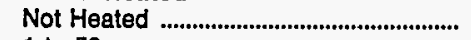 & 100 & $\mathbf{Q}$ & 15 & 10 & 2 & 31 & 2 & 8 & 11 & 22 \\
\hline 1 to 50 & 100 & 2 & 7 & 5 & 1 & 36 & 2 & 12 & 20 & 16 \\
\hline 51 to 99 & 100 & 3 & 10 & 11 & 1 & 36 & 2 & 6 & 16 & 17 \\
\hline 100 & 100 & 4 & 10 & 10 & 1 & 38 & 2 & 6 & 13 & 16 \\
\hline \multicolumn{11}{|l|}{ Percent Cooled } \\
\hline Not Cooled .......... & 100 & 2 & NC & 10 & 1 & 41 & 2 & 7 & 17 & 20 \\
\hline 1 to 50 & 100 & 3 & 7 & 6 & 1 & 37 & 2 & 7 & 16 & 20 \\
\hline 51 to 99 & 100 & 3 & 12 & 11 & 1 & 38 & 2 & 7 & 13 & 14 \\
\hline 100 & 100 & 4 & 12 & 11 & $i$ & 36 & 2 & 6 & 13 & 16 \\
\hline
\end{tabular}

See footnotes at end of table. 
Table B6. End-Use Consumption Percentages for Electricity, 1989 (Continued)

\begin{tabular}{|c|c|c|c|c|c|c|c|c|c|c|}
\hline \multirow[b]{2}{*}{$\begin{array}{l}\text { Bullding } \\
\text { Characterlstles }\end{array}$} & \multicolumn{10}{|c|}{ Percent of Electricity Consumption } \\
\hline & Total & $\begin{array}{l}\text { Space } \\
\text { Heatlng }\end{array}$ & Cooling & $\begin{array}{l}\text { Ventll- } \\
\text { ation }\end{array}$ & $\begin{array}{l}\text { Water } \\
\text { Heating }\end{array}$ & Ughting & Cooking & $\begin{array}{l}\text { Retrig- } \\
\text { eration }\end{array}$ & $\begin{array}{l}\text { Offlce } \\
\text { Equip- } \\
\text { ment }\end{array}$ & Other \\
\hline \multicolumn{11}{|l|}{$\begin{array}{l}\text { Heatling Equipment } \\
\text { (Solely or in Comblnation) }\end{array}$} \\
\hline 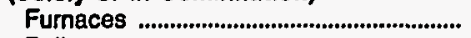 & 100 & 4 & 9 & 7 & 1 & 36 & 2 & 9 & 16 & 16 \\
\hline 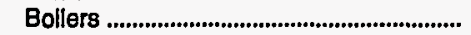 & 100 & 2 & 10 & 12 & 1 & 41 & 2 & 6 & 12 & 15 \\
\hline Individual Space Heaters ........................... & 100 & 5 & 8 & 9 & 1 & 38 & 2 & 6 & 14 & 17 \\
\hline 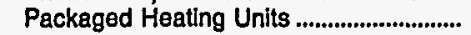 & 100 & 3 & 11 & 8 & 1 & 36 & $\overline{2}$ & 6 & 13 & 19 \\
\hline Heat Pumps ................................................... & 100 & 6 & 12 & 10 & 1 & 35 & 2 & 7 & 13 & 14 \\
\hline Alr Ducts .......................................................... & 100 & 3 & 10 & 10 & $i$ & 38 & 2 & 6 & 14 & 16 \\
\hline Heating or Reheating Coils .......................... & 100 & 3 & 9 & 12 & • & 39 & $i$ & 5 & 12 & 18 \\
\hline $\begin{array}{l}\text { Fan-Coll Units } \\
\text { Steam or Hot Water Radiators }\end{array}$ & 100 & 2 & 9 & 12 & - & 40 & 1 & 5 & 11 & 19 \\
\hline or Baseboards ............................................ & 100 & 2 & 7 & 13 & - & 42 & 2 & 6 & 12 & 15 \\
\hline Other & 100 & $\mathbf{Q}$ & 12 & 15 & 1 & 35 & 2 & 8 & 12 & 12 \\
\hline \multicolumn{11}{|l|}{$\begin{array}{l}\text { Coolling Equ/pment } \\
\text { (Solely or In Combination) }\end{array}$} \\
\hline Central Chillers .................................................. & 100 & 3 & 11 & 14 & 1 & 37 & 1 & 5 & 12 & 16 \\
\hline Individual Air Conditioners ............................. & 100 & 4 & 10 & 9 & 1 & 36 & 2 & 7 & 12 & 20 \\
\hline Packaged Coolling Units .................................. & 100 & 3 & 11 & 9 & $i$ & 36 & 2 & 7 & 14 & 17 \\
\hline Heat Pumps ..................................................... & 100 & 6 & 10 & 9 & 1 & 32 & 2 & 7 & 14 & 20 \\
\hline Alr Ducts ............................................................ & 100 & 3 & 11 & 10 & $i$ & 37 & $\overline{2}$ & 6 & 13 & 16 \\
\hline 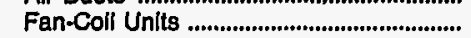 & 100 & 3 & 10 & 14 & - & 38 & 1 & 5 & 12 & 16 \\
\hline Other & 100 & 1 & 13 & 8 & 1 & 46 & 1 & 4 & 13 & 13 \\
\hline \multicolumn{11}{|l|}{ Yoar Maln Central Chller Installed } \\
\hline 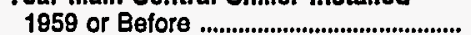 & 100 & $\mathbf{Q}$ & 12 & 18 & 1 & 35 & 1 & 4 & 11 & 9 \\
\hline 1860 to 1969 & 100 & 1 & 10 & 11 & - & 36 & 1 & 4 & 10 & 26 \\
\hline 1970 to 1979 & 100 & 2 & 11 & 14 & 1 & 39 & $i$ & 5 & 12 & 16 \\
\hline 1980 to 1888 & $\begin{array}{l}100 \\
100\end{array}$ & 4 & $\begin{array}{l}11 \\
12\end{array}$ & 17 & 10 & $\begin{array}{l}38 \\
39\end{array}$ & 2 & 5 & 12 & 11 \\
\hline \multicolumn{11}{|l|}{$\begin{array}{l}\text { Year Packaged Coolling System } \\
\text { Installed }\end{array}$} \\
\hline 1959 or Before .................................................... & 100 & $\mathbf{Q}$ & 11 & 12 & $\mathbf{Q}$ & 45 & 1 & 4 & 12 & 15 \\
\hline 1880 to 1869 & 100 & 2 & $\theta$ & 8 & $\overline{1}$ & 36 & $i$ & 5 & 13 & 26 \\
\hline 1970 to 1979 & 100 & 4 & 12 & 10 & 1 & 37 & 2 & 7 & 13 & 13 \\
\hline 1980 to 1888 & 100 & 3 & 11 & 9 & $i$ & 36 & 2 & 8 & 15 & 16 \\
\hline 1987 to 1989 & 100 & 1 & 12 & 10 & 1 & 34 & 2 & 8 & 12 & 18 \\
\hline \multicolumn{11}{|l|}{$\begin{array}{l}\text { Computer Area with Soparate } \\
\text { Alr-Conditloning System }\end{array}$} \\
\hline Present in Buliding ....................................... & 100 & 2 & 10 & 12 & - & 38 & 1 & 5 & 15 & 17 \\
\hline 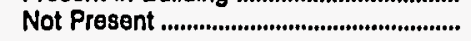 & 100 & 5 & 11 & 8 & 1 & 36 & 3 & 8 & 13 & 16 \\
\hline \multicolumn{11}{|l|}{$\begin{array}{l}\text { LIOHTING ANO REFRIGERATION } \\
\text { Percent Lt When OPen }\end{array}$} \\
\hline 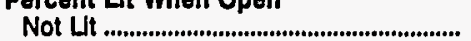 & 100 & $\mathbf{Q}$ & 5 & 5 & 1 & 24 & 1 & 8 & 15 & 41 \\
\hline 1 to 50 & 100 & 7 & 10 & 11 & 2 & 25 & 2 & 8 & 16 & 18 \\
\hline 51 to 99 & 100 & 3 & 10 & 10 & $\overline{1}$ & 36 & 2 & 5 & 14 & 19 \\
\hline 100 & 100 & 3 & 10 & 10 & 1 & 39 & $\overline{2}$ & 7 & 13 & 14 \\
\hline \multicolumn{11}{|l|}{ Percent Lit When Closed } \\
\hline 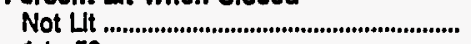 & 100 & 5 & 11 & $\theta$ & 1 & 33 & 2 & 7 & 14 & 18 \\
\hline 1 to 50 & 100 & 3 & 8 & 10 & $i$ & 39 & 2 & 7 & 14 & 16 \\
\hline 61 to 99 & 100 & 1 & 11 & 13 & 1 & 40 & 3 & 6 & 11 & 14 \\
\hline 100 & 100 & $Q$ & 18 & 12 & $\mathbf{Q}$ & 35 & 5 & $\mathbf{Q}$ & 3 & 9 \\
\hline \multicolumn{11}{|l|}{$\begin{array}{l}\text { Lghtling Equlpment } \\
\text { (Sololy or in Combinetlon) }\end{array}$} \\
\hline Incandescent Lamps ................................... & 100 & 4 & 10 & 10 & 1 & 39 & 2 & 7 & 12 & 15 \\
\hline 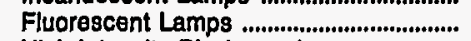 & 100 & 3 & 10 & 10 & $i$ & 37 & 2 & 7 & 14 & 16 \\
\hline Hlgh-Intensity Dlscharge Lamps ............ & 100 & 3 & 9 & 10 & - & 40 & 1 & 6 & 12 & 18 \\
\hline Other Lamps ...................................................... & 100 & 2 & 13 & 13 & 1 & 41 & 2 & 8 & 9 & 11 \\
\hline Hligh-Effleiency Ballasts ............................... & 100 & 4 & 10 & 11 & 1 & 35 & 2 & 7 & 15 & 16 \\
\hline
\end{tabular}

See footnotes at end of table. 
Table B6. End-Use Consumption Percentages for Electricity, 1989 (Continued)

\begin{tabular}{|c|c|c|c|c|c|c|c|c|c|c|}
\hline \multirow[b]{2}{*}{$\begin{array}{l}\text { Building } \\
\text { Characteristics }\end{array}$} & \multicolumn{10}{|c|}{ Percent of Electricity Consumption } \\
\hline & Total & $\begin{array}{l}\text { Space } \\
\text { Heating }\end{array}$ & Coollng & $\begin{array}{l}\text { Ventil- } \\
\text { ation }\end{array}$ & $\begin{array}{l}\text { Water } \\
\text { Heating }\end{array}$ & Lighting & Cooking & $\begin{array}{l}\text { Refrig- } \\
\text { eration }\end{array}$ & $\begin{array}{l}\text { Offlce } \\
\text { Equip- } \\
\text { ment }\end{array}$ & Other \\
\hline \multicolumn{11}{|l|}{$\begin{array}{l}\text { Refrigeration Equipment } \\
\text { (Solely or In Combination) } \\
\text { Commercial }\end{array}$} \\
\hline Refrigeration Units ...................................... & 100 & 2 & 10 & 11 & 1 & 37 & $\mathbf{3}$ & 9 & 11 & 17 \\
\hline \multicolumn{10}{|l|}{ Residential } & 17 \\
\hline 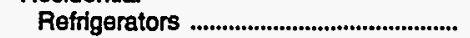 & 100 & 3 & 10 & 10 & 1 & 39 & 1 & 6 & 14 & 16 \\
\hline 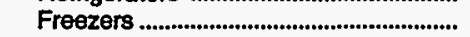 & 100 & $\mathbf{3}$ & 11 & 10 & 1 & 38 & 3 & 6 & 10 & 19 \\
\hline 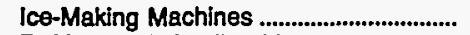 & 100 & 3 & 11 & 11 & 1 & 36 & 3 & 7 & 11 & 16 \\
\hline Refrigerated Vending Machines ............... & 100 & 3 & 10 & 10 & $i$ & 38 & 2 & 7 & 13 & 15 \\
\hline 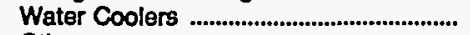 & 100 & 3 & 10 & 10 & 1 & 38 & 1 & 6 & 13 & 17 \\
\hline Other & 100 & $\mathbf{Q}$ & 11 & 7 & 1 & 30 & 2 & 6 & 12 & $\mathbf{Q}$ \\
\hline \multicolumn{11}{|l|}{$\begin{array}{l}\text { ENERGV MANAGEMENT } \\
\text { Occupant Control }\end{array}$} \\
\hline 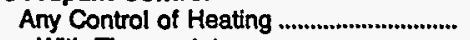 & 100 & 4 & 11 & 9 & 1 & 37 & 2 & 6 & 14 & 16 \\
\hline 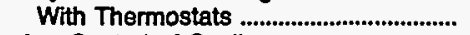 & 100 & 4 & 11 & 9 & 1 & 37 & 2 & 6 & 14 & 16 \\
\hline 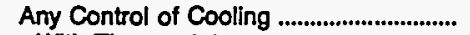 & 100 & 4 & 12 & 9 & 1 & 36 & 2 & 6 & 14 & 16 \\
\hline 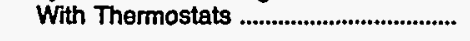 & 100 & 4 & 12 & 9 & 1 & 36 & 2 & 6 & 13 & 16 \\
\hline \multicolumn{11}{|l|}{ Reduced Use Durting Ott-Hours } \\
\hline 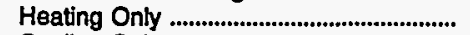 & 100 & 3 & 4 & 10 & 1 & 40 & 2 & 7 & 15 & 18 \\
\hline 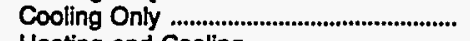 & 100 & 4 & 12 & 9 & 1 & 34 & 2 & 5 & 15 & 18 \\
\hline 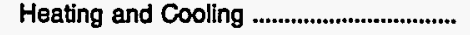 & 100 & 4 & 10 & 11 & 1 & 37 & 2 & 6 & 14 & 16 \\
\hline \multicolumn{11}{|l|}{$\begin{array}{l}\text { Computerized Energy Management } \\
\text { and Control System }\end{array}$} \\
\hline 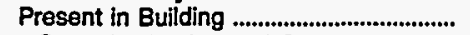 & 100 & 2 & 10 & 13 & * & 40 & 2 & 6 & 11 & 15 \\
\hline Controls Heating and Cooling ............... & 100 & 2 & 10 & 13 & * & 40 & 2 & 6 & 11 & 15 \\
\hline 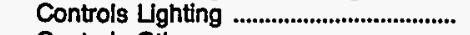 & 100 & 2 & 9 & 10 & " & 40 & 2 & 7 & 11 & 19 \\
\hline 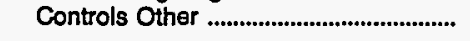 & 100 & 2 & 13 & 12 & * & 39 & 2 & 6 & 10 & 15 \\
\hline \multicolumn{11}{|l|}{ Other Energy Management } \\
\hline $\begin{array}{l}\text { Regular HVAC Maintenance .............................. } \\
\text { Participated in Utility }\end{array}$ & 100 & 3 & 10 & 10 & 1 & 37 & 2 & 7 & 13 & 16 \\
\hline 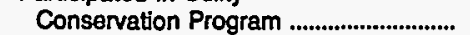 & 100 & 5 & 10 & 13 & 1 & 37 & 2 & 7 & 12 & 14 \\
\hline
\end{tabular}

$-=$ Value rounds to zero in the units displayed.

NC $=$ No cases in responding sample.

$Q=$ Data withheld because the Relative Standard Error (RSE) was greater than 50 percent, or data were reported for fewer than 20 buildings.

Notes: - See Glossary for explanation of abbreviations and definitions of terms used in this report. - Because of rounding, data may not sum to totals.

Source: Energy Information Administration, Office of Energy Markets and End Use, Forms ElA-871A through F of the 1989 Commercial Buildings Energy Consumption Survey. 


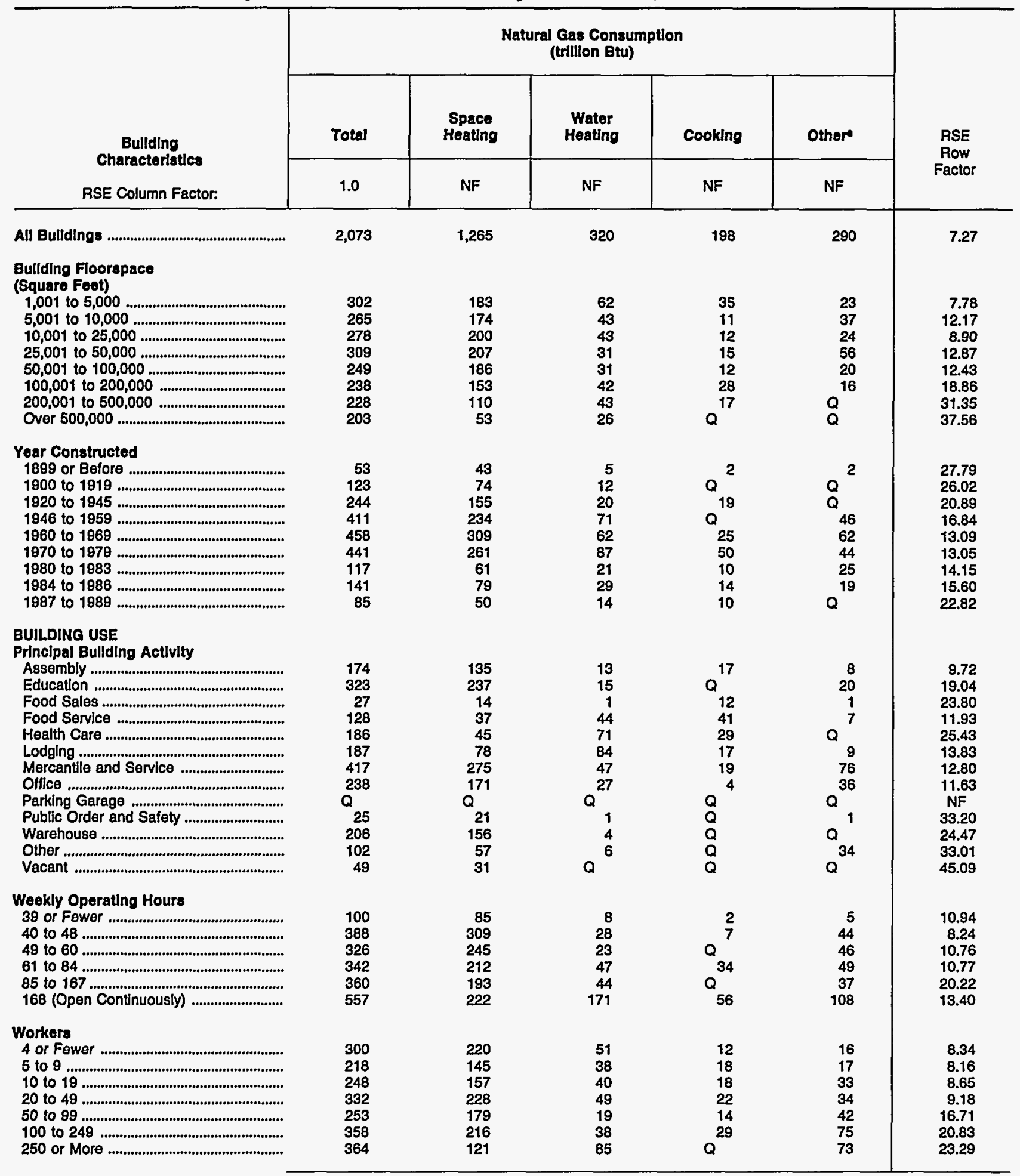

See footnotes at end of table. 
Table B7. Consumption of Natural Gas by End Uses, 1989 (Continued)

\begin{tabular}{|c|c|c|c|c|c|c|}
\hline \multirow{3}{*}{$\begin{array}{c}\text { Building } \\
\text { Characteristlos } \\
\text { RSE Column Factor: }\end{array}$} & \multicolumn{5}{|c|}{$\begin{array}{l}\text { Natural Gas Consumption } \\
\text { (trillion Btu) }\end{array}$} & \multirow{3}{*}{$\begin{array}{l}\text { RSE } \\
\text { Row } \\
\text { Factor }\end{array}$} \\
\hline & Total & $\begin{array}{c}\text { Space } \\
\text { Heating }\end{array}$ & $\begin{array}{c}\text { Water } \\
\text { Heating }\end{array}$ & Cooking & Other" & \\
\hline & 1.0 & NF & NF & NF & NF & \\
\hline \multicolumn{7}{|l|}{$\begin{array}{l}\text { Ownershlp and Occupancy } \\
\text { Nongovernment Owned }\end{array}$} \\
\hline Nongovernment Owned .................................. & 1,601 & 950 & 257 & 130 & 264 & 8.01 \\
\hline 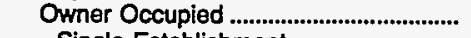 & 1,292 & 749 & 213 & 103 & 228 & 8.64 \\
\hline Single Establishment ................................ & 1,083 & 622 & 181 & 83 & 197 & 10.40 \\
\hline Multiple Establishment .............................. & 210 & 127 & 32 & 20 & 31 & 11.20 \\
\hline Nonowner Occupied .................................. & 309 & 201 & 44 & 27 & 36 & 14.72 \\
\hline 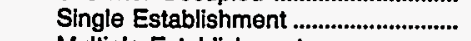 & 176 & 123 & 18 & 15 & 20 & 22.17 \\
\hline 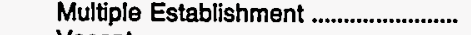 & 102 & 62 & 21 & 12 & 7 & 16.89 \\
\hline Vacant & & 17 & $\mathbf{Q}$ & $\mathbf{Q}$ & $\mathbf{Q}$ & $\mathrm{NF}$ \\
\hline 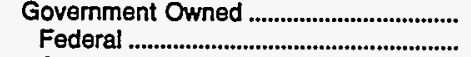 & $a^{472}$ & $\begin{array}{r}315 \\
16\end{array}$ & $\begin{array}{r}63 \\
5\end{array}$ & & $Q^{26}$ & $\begin{array}{c}15.34 \\
\mathrm{NF}\end{array}$ \\
\hline State & 112 & 79 & 18 & 4 & 11 & 21.52 \\
\hline 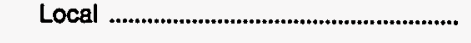 & & 220 & 40 & 17 & 13 & 12.64 \\
\hline \multicolumn{7}{|l|}{ Multibullding Facllity } \\
\hline Not on Multibuilding Facility ....................... & 1,168 & 765 & 175 & 80 & 147 & 7.35 \\
\hline $\begin{array}{l}\text { Part of Multibuilding Facility ......................... } \\
\text { On Facility with Central }\end{array}$ & 905 & 500 & 145 & 118 & 143 & 12.68 \\
\hline 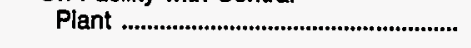 & 423 & 182 & 66 & Q & 90 & 24.78 \\
\hline \multicolumn{7}{|l|}{$\begin{array}{l}\text { Percent Vacant at Least Three } \\
\text { Months }\end{array}$} \\
\hline 0 & 1,562 & 988 & 257 & 119 & 198 & 7.65 \\
\hline 1 to 50 & $\mathbf{Q}^{324}$ & $\begin{array}{r}178 \\
47\end{array}$ & $\begin{array}{r}41 \\
5\end{array}$ & $Q^{29}$ & $\begin{array}{r}76 \\
4\end{array}$ & $\begin{array}{c}17.42 \\
\mathrm{NF}\end{array}$ \\
\hline 100 & 82 & 52 & 16 & 1 & $\mathbf{Q}$ & 28.05 \\
\hline \multicolumn{7}{|l|}{ Months In Use Out of Past 12 Months } \\
\hline 0 to 8 & 72 & 42 & 16 & 9 & 4 & 18.41 \\
\hline 9 to 11 & 138 & 106 & 16 & 2 & Q & 21.43 \\
\hline 12 & 1,863 & 1,117 & 288 & 187 & 271 & 7.43 \\
\hline \multicolumn{7}{|l|}{$\begin{array}{l}\text { LOCATION } \\
\text { Census Reglon } \\
\text { Northeast }\end{array}$} \\
\hline 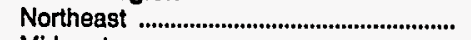 & 353 & 235 & 52 & 30 & $\mathbf{Q}$ & 17.15 \\
\hline 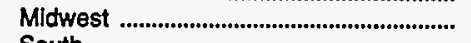 & 831 & 579 & 101 & 46 & 105 & 10.12 \\
\hline South & 498 & 263 & 84 & 42 & 108 & 17.83 \\
\hline 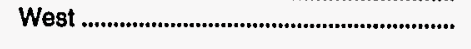 & 391 & 189 & 82 & $\mathbf{Q}$ & 41 & 14.77 \\
\hline \multirow{2}{*}{\multicolumn{7}{|c|}{$\begin{array}{l}\text { Census Division } \\
\text { Northeast }\end{array}$}} \\
\hline & 39 & 19 & $\mathbf{Q}$ & & & \\
\hline 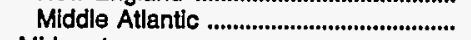 & 314 & 215 & 40 & 26 & $0^{4}$ & $\begin{array}{l}22.84 \\
19.38\end{array}$ \\
\hline \multicolumn{7}{|l|}{ Midwest } \\
\hline 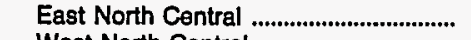 & 561 & 398 & 73 & 36 & 54 & 12.18 \\
\hline 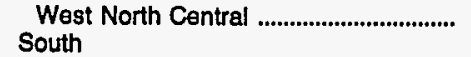 & 270 & \multirow{2}{*}{\multicolumn{4}{|c|}{ South }} & 20.01 \\
\hline 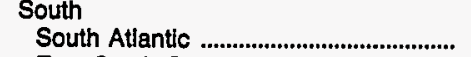 & 198 & 106 & 24 & & & \\
\hline 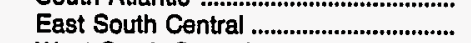 & 126 & 64 & $Q$ & $\begin{array}{r}10 \\
9\end{array}$ & 28 & $\begin{array}{l}31.50 \\
29.22\end{array}$ \\
\hline 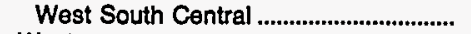 & 174 & 94 & 34 & 19 & 27 & 19.57 \\
\hline \multicolumn{7}{|l|}{ West } \\
\hline 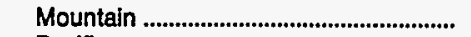 & 197 & 97 & 28 & $\mathbf{Q}$ & 13 & 25.75 \\
\hline 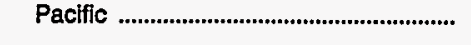 & 195 & 91 & 54 & 21 & 28 & 14.77 \\
\hline \multicolumn{7}{|l|}{ Metropolltan Status } \\
\hline Metropolitan & 1,608 & 939 & 254 & 176 & 239 & 7.68 \\
\hline 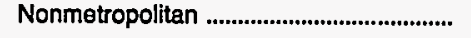 & 465 & 326 & 66 & 22 & 51 & 18.83 \\
\hline
\end{tabular}

See footnotes at end of table. 
Table B7. Consumption of Natural Gas by End Uses, 1989 (Continued)

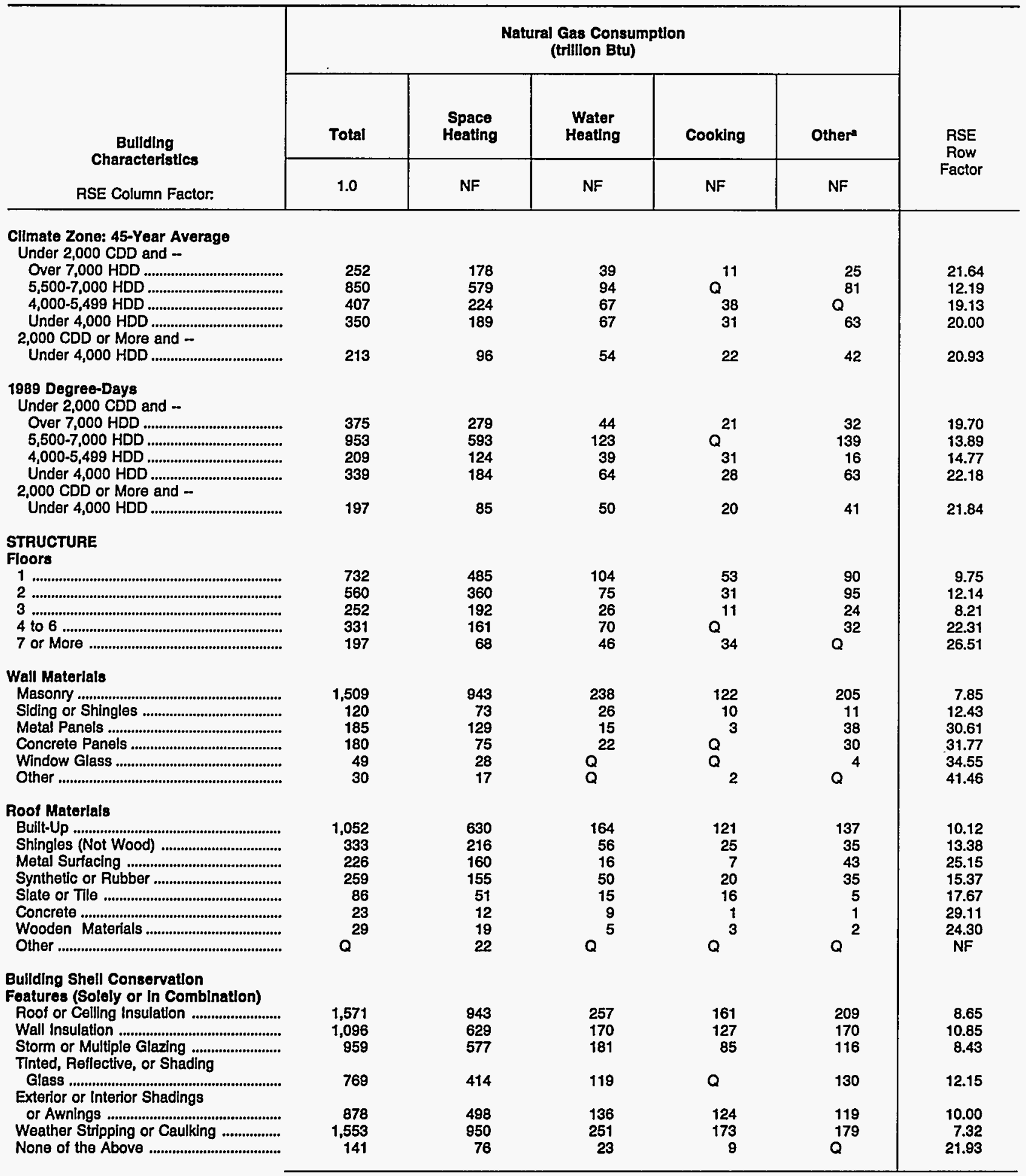

See footnotes at end of table. 
Table B7. Consumption of Natural Gas by End Use, 1989 (Continued)

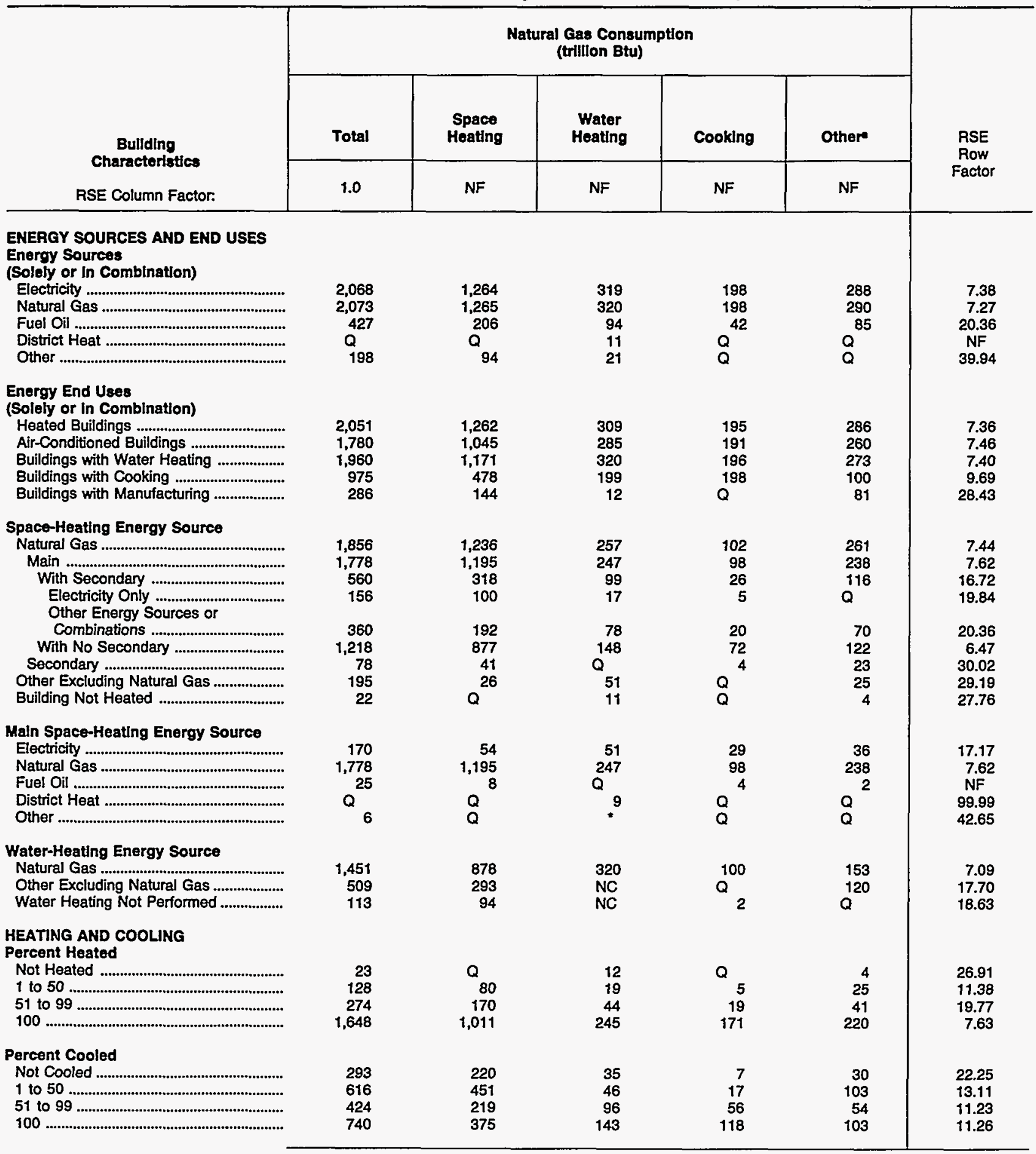

See footnotes at end of table. 
Table B7. Consumption of Natural Gas by End Use, 1989 (Continued)

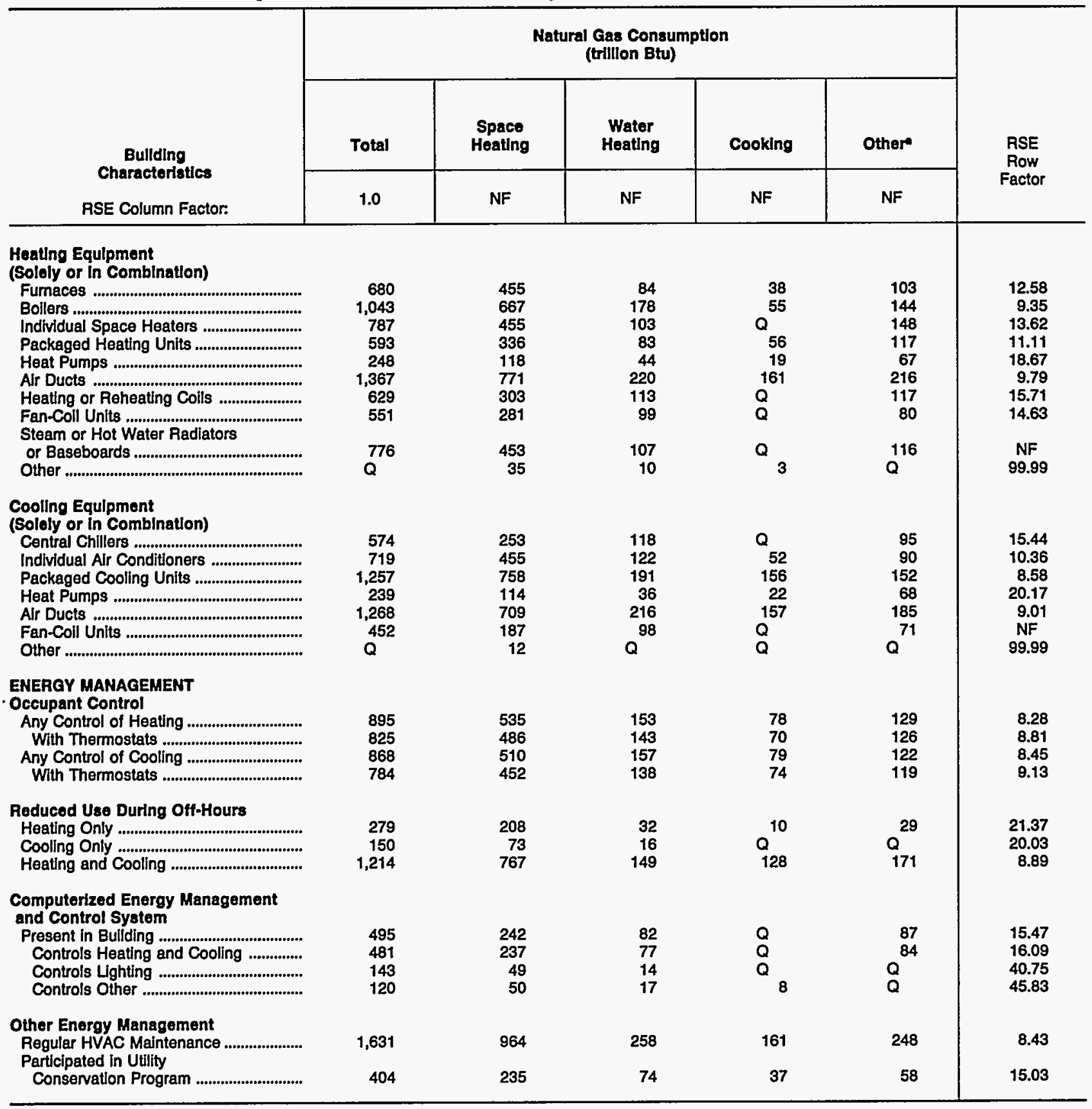

- Includes cooling.

- = Value rounds to zero in the units displayed.

$\mathrm{NC}=$ No cases in responding sample.

NF = No applicable RSE row/column factor.

$Q=$ Data withheld because the Relative Standard Error (RSE) was greater than 50 percent, or data were reported for fewer than 20 buildings.

Notes: - To obtain the RSE percentage for any table cell, multiply the corresponding RSE column and RSE row factors. - See Glossary for explanation of abbreviations and definitions of terms used in this report. Because of rounding, data may not sum to totals.

Source: Energy Information Administration, Office of Energy Markets and End Use, Forms EIA-871A through F of the 1989 Commercial Buildings Energy Consumption Survey. 


\begin{tabular}{|c|c|c|c|c|c|c|}
\hline \multirow{3}{*}{$\begin{array}{c}\text { Building } \\
\text { Characteristics } \\
\text { RSE Column Factor: }\end{array}$} & \multicolumn{5}{|c|}{$\begin{array}{l}\text { Energy Intensity for Natural Gas } \\
\text { (thousand Btu per sq. ft.) }\end{array}$} & \multirow{3}{*}{$\begin{array}{l}\text { RSE } \\
\text { Row } \\
\text { Factor }\end{array}$} \\
\hline & \multirow[t]{2}{*}{ Total } & \multirow{2}{*}{$\begin{array}{c}\begin{array}{c}\text { Space } \\
\text { Heating }\end{array} \\
\text { NF }\end{array}$} & \multirow{2}{*}{$\begin{array}{c}\begin{array}{c}\text { Water } \\
\text { Heating }\end{array} \\
\text { NF }\end{array}$} & \multirow{2}{*}{$\begin{array}{c}\text { Cooking } \\
\text { NF }\end{array}$} & \multirow{2}{*}{$\begin{array}{c}\text { Other } \\
\text { NF }\end{array}$} & \\
\hline & & & & & & \\
\hline All Buildings & 50.4 & 30.7 & 7.8 & 4.8 & 7.0 & 5.91 \\
\hline $\begin{array}{l}\text { Bullding Floorspace } \\
\text { (Square Feet) } \\
1,001 \text { to } 5,000 \\
5,001 \text { to } 10,000 \\
10,001 \text { to } 25,000 \\
25,001 \text { to } 50,000 \\
50,001 \text { to } 100,000 \\
100,001 \text { to } 200,000 \\
200,001 \text { to } 500,000 \\
\text { Over } 500,000\end{array}$ & $\begin{array}{l}88.3 \\
67.1 \\
48.3 \\
56.7 \\
40.1 \\
39.6 \\
45.2 \\
38.5\end{array}$ & $\begin{array}{l}53.5 \\
43.9 \\
34.7 \\
38.0 \\
29.9 \\
25.4 \\
21.8 \\
10.0\end{array}$ & $\begin{array}{r}18.0 \\
11.0 \\
7.4 \\
5.7 \\
5.0 \\
7.0 \\
8.4 \\
4.9\end{array}$ & $\begin{array}{r}10.2 \\
2.9 \\
2.0 \\
2.7 \\
1.9 \\
4.6 \\
3.3 \\
Q^{3}\end{array}$ & $\begin{array}{r}6.7 \\
9.3 \\
4.1 \\
10.2 \\
3.3 \\
2.6 \\
Q \\
Q\end{array}$ & $\begin{array}{r}4.96 \\
11.63 \\
6.51 \\
11.69 \\
9.67 \\
18.17 \\
26.13 \\
29.95\end{array}$ \\
\hline $\begin{array}{l}\text { Year Constructed } \\
1899 \text { or Before } \\
1900 \text { to } 1919 \\
1920 \text { to } 1945 \\
1946 \text { to } 1959 \\
1960 \text { to } 1969 \\
1970 \text { to } 1979 \\
1980 \text { to } 1983 \\
1984 \text { to } 1986 \\
1987 \text { to } 1989\end{array}$ & $\begin{array}{l}52.3 \\
40.2 \\
42.5 \\
56.8 \\
54.0 \\
54.4 \\
53.6 \\
40.9 \\
45.4\end{array}$ & $\begin{array}{l}42.6 \\
24.0 \\
27.0 \\
32.3 \\
36.4 \\
32.2 \\
27.8 \\
22.9 \\
26.8\end{array}$ & $\begin{array}{r}5.1 \\
3.8 \\
3.4 \\
9.8 \\
7.3 \\
10.7 \\
9.4 \\
8.4 \\
7.7\end{array}$ & $\begin{array}{c}Q^{2.3} \\
Q^{3.4} \\
3.0 \\
6.1 \\
4.8 \\
4.0 \\
5.5\end{array}$ & $\begin{array}{l}Q^{2.3} \\
Q \\
6.4 \\
7.3 \\
5.4 \\
11.6 \\
Q^{5.6}\end{array}$ & $\begin{array}{l}19.97 \\
30.69 \\
15.03 \\
11.28 \\
10.14 \\
13.87 \\
12.66 \\
18.94 \\
19.37\end{array}$ \\
\hline 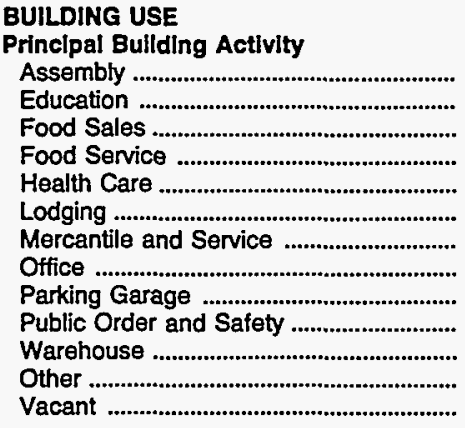 & $\begin{array}{r}40.4 \\
48.7 \\
49.8 \\
156.4 \\
116.3 \\
73.7 \\
47.4 \\
33.0 \\
37.6 \\
55.7 \\
40.2 \\
109.1 \\
Q\end{array}$ & $\begin{array}{l}31.4 \\
35.6 \\
24.6 \\
44.8 \\
28.4 \\
30.7 \\
31.3 \\
23.7 \\
28.2 \\
48.8 \\
30.5 \\
61.2 \\
16.2\end{array}$ & \begin{tabular}{r}
3.0 \\
2.2 \\
1.5 \\
53.3 \\
44.5 \\
33.0 \\
5.4 \\
3.7 \\
$Q^{Q}$ \\
$Q$ \\
\multicolumn{2}{c}{.7} \\
$Q^{6.3}$
\end{tabular} & $\begin{array}{l}Q^{4.0} \\
22.6 \\
49.9 \\
18.2 \\
6.6 \\
2.2 \\
.6 \\
Q \\
Q \\
Q \\
Q \\
Q\end{array}$ & $\begin{array}{l}1.9 \\
3.0 \\
1.0 \\
8.5 \\
Q^{3.4} \\
8.6 \\
4.9 \\
Q^{2.6} \\
Q \\
36.9 \\
Q\end{array}$ & $\begin{array}{r}11.33 \\
11.47 \\
16.88 \\
9.53 \\
10.48 \\
11.94 \\
10.21 \\
11.19 \\
47.02 \\
33.93 \\
22.86 \\
18.36 \\
\text { NF }\end{array}$ \\
\hline 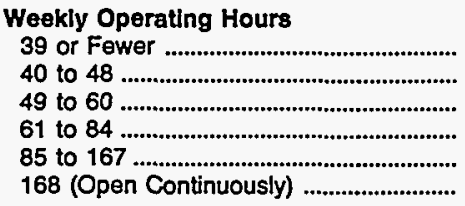 & $\begin{array}{l}38.0 \\
42.3 \\
38.5 \\
43.0 \\
54.8 \\
87.7\end{array}$ & $\begin{array}{l}32.4 \\
33.7 \\
28.9 \\
26.6 \\
29.3 \\
34.9\end{array}$ & $\begin{array}{r}2.9 \\
3.0 \\
2.7 \\
5.9 \\
6.7 \\
26.8\end{array}$ & $\begin{array}{r}.9 \\
Q^{.8} \\
4.3 \\
13.1 \\
8.8\end{array}$ & $\begin{array}{r}1.8 \\
4.8 \\
5.5 \\
6.2 \\
5.7 \\
17.1\end{array}$ & $\begin{array}{r}9.06 \\
8.15 \\
9.40 \\
11.28 \\
16.39 \\
11.18\end{array}$ \\
\hline 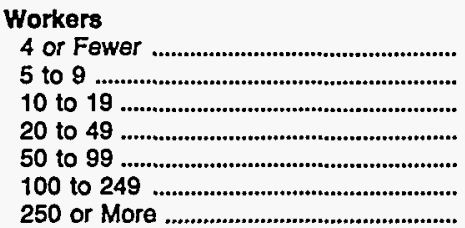 & $\begin{array}{l}44.6 \\
42.0 \\
55.7 \\
50.2 \\
47.3 \\
71.0 \\
46.9\end{array}$ & $\begin{array}{l}32.7 \\
28.0 \\
35.3 \\
34.4 \\
33.4 \\
42.8 \\
15.5\end{array}$ & $\begin{array}{r}7.6 \\
7.3 \\
8.9 \\
7.3 \\
3.6 \\
7.6 \\
10.9\end{array}$ & $\begin{array}{l}1.8 \\
3.4 \\
4.0 \\
3.3 \\
2.6 \\
5.8 \\
Q\end{array}$ & $\begin{array}{r}2.4 \\
3.3 \\
7.4 \\
5.2 \\
7.8 \\
14.8 \\
9.4\end{array}$ & $\begin{array}{r}11.29 \\
9.37 \\
9.10 \\
9.29 \\
16.09 \\
14.03 \\
14.93\end{array}$ \\
\hline
\end{tabular}

See footnote at end of table. 


\begin{tabular}{|c|c|c|c|c|c|c|}
\hline \multirow{3}{*}{$\begin{array}{c}\text { Building } \\
\text { Characteristics } \\
\text { RSE Column Factor: }\end{array}$} & \multicolumn{5}{|c|}{$\begin{array}{l}\text { Energy Intensity for Natural Gas } \\
\text { (thousand Btu per sq. ft.) }\end{array}$} & \multirow{3}{*}{$\begin{array}{l}\text { RSE } \\
\text { Row } \\
\text { Factor }\end{array}$} \\
\hline & Total & $\begin{array}{l}\text { Space } \\
\text { Heatling }\end{array}$ & $\begin{array}{l}\text { Water } \\
\text { Heating }\end{array}$ & Cooking & Other" & \\
\hline & 1.0 & NF & NF & NF & NF & \\
\hline 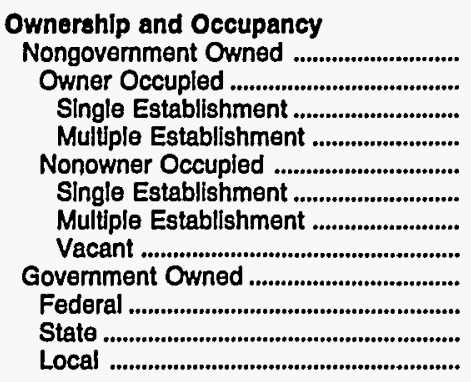 & $\begin{array}{l}50.5 \\
54.7 \\
63.1 \\
32.4 \\
38.3 \\
41.0 \\
29.0 \\
Q \\
50.0 \\
76.3 \\
48.3 \\
46.8\end{array}$ & $\begin{array}{l}30.0 \\
31.7 \\
36.2 \\
19.6 \\
24.9 \\
28.7 \\
17.6 \\
60.2 \\
33.4 \\
17.3 \\
34.0 \\
35.5\end{array}$ & $\begin{array}{r}8.1 \\
9.0 \\
10.6 \\
4.9 \\
5.4 \\
4.3 \\
5.9 \\
0 \\
6.7 \\
5.4 \\
7.8 \\
6.4\end{array}$ & $\begin{array}{l}4.1 \\
4.4 \\
4.8 \\
3.1 \\
3.4 \\
3.5 \\
3.4 \\
Q \\
Q \\
51.8 \\
1.7 \\
2.7\end{array}$ & $\begin{array}{r}8.3 \\
9.6 \\
11.5 \\
4.7 \\
4.5 \\
4.6 \\
2.1 \\
Q \\
2.8 \\
Q \\
4.8 \\
2.1\end{array}$ & $\begin{array}{r}6.61 \\
7.13 \\
8.68 \\
13.59 \\
14.39 \\
20.79 \\
15.42 \\
\mathrm{NF} \\
10.45 \\
17.88 \\
18.59 \\
10.58\end{array}$ \\
\hline $\begin{array}{l}\text { Multubullding Facllity } \\
\text { Not on Multibullding Facility ...................... } \\
\text { Part of Multibuilding Facility ...................... } \\
\text { On Facillty with Central }\end{array}$ & $\begin{array}{l}44.7 \\
60.3 \\
91.4\end{array}$ & $\begin{array}{l}29.3 \\
33.3 \\
39.4\end{array}$ & $\begin{array}{r}6.7 \\
9.6 \\
14.3\end{array}$ & $\begin{array}{l}3.1 \\
7.9\end{array}$ & $\begin{array}{r}5.6 \\
9.5 \\
19.5\end{array}$ & $\begin{array}{r}7.06 \\
7.85 \\
13.32\end{array}$ \\
\hline $\begin{array}{l}\text { Porcent Vacent at Least Three } \\
\text { Months } \\
0 \\
100 \\
51 \text { to } 99\end{array}$ & $\begin{array}{l}56.9 \\
35.1 \\
36.8 \\
51.6\end{array}$ & $\begin{array}{l}36.0 \\
19.3 \\
16.6 \\
32.9\end{array}$ & $\begin{array}{r}9.4 \\
4.5 \\
1.7 \\
10.3\end{array}$ & $\begin{array}{l}4.3 \\
Q^{3.1} \\
.7\end{array}$ & $\begin{array}{l}7.2 \\
8.3 \\
1.3 \\
Q\end{array}$ & $\begin{array}{r}6.21 \\
15.18 \\
36.19 \\
27.59\end{array}$ \\
\hline 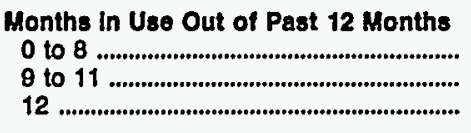 & $\begin{array}{l}41.3 \\
52.4 \\
50.7\end{array}$ & $\begin{array}{l}24.2 \\
40.2 \\
30.4\end{array}$ & $\begin{array}{l}9.4 \\
5.9 \\
7.8\end{array}$ & $\begin{array}{r}5.3 \\
.7 \\
5.1\end{array}$ & $Q_{7.4}^{2.4}$ & $\begin{array}{r}16.22 \\
17.23 \\
6.57\end{array}$ \\
\hline 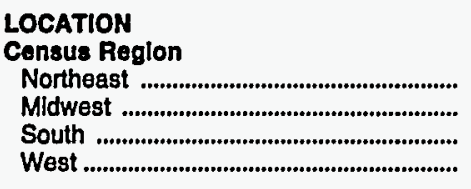 & $\begin{array}{l}41.5 \\
64.8 \\
42.7 \\
48.0\end{array}$ & $\begin{array}{l}27.5 \\
45.1 \\
22.6 \\
23.1\end{array}$ & $\begin{array}{r}6.1 \\
7.9 \\
7.2 \\
10.1\end{array}$ & $\begin{array}{l}3.5 \\
3.6 \\
\mathbf{Q}\end{array}$ & $\begin{array}{l}4.2 \\
8.2 \\
9.3 \\
5.1\end{array}$ & $\begin{array}{r}15.13 \\
8.71 \\
13.83 \\
7.97\end{array}$ \\
\hline $\begin{array}{l}\text { Census Divislon } \\
\text { Northeast }\end{array}$ & & & & & & \\
\hline $\begin{array}{l}\text { New England .............................................. } \\
\text { Mlddle Attantic .......................................... } \\
\text { Midwest }\end{array}$ & $\begin{array}{l}31.8 \\
43.1\end{array}$ & $\begin{array}{l}15.7 \\
29.5\end{array}$ & $Q_{5.6}$ & $\mathrm{Q}_{3.6}$ & $\begin{array}{l}3.6 \\
4.3\end{array}$ & $\begin{array}{l}24.95 \\
17.07\end{array}$ \\
\hline 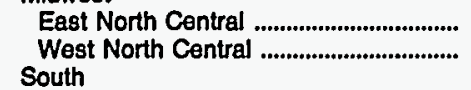 & $\begin{array}{l}63.8 \\
67.2\end{array}$ & $\begin{array}{l}45.3 \\
44.9\end{array}$ & $\begin{array}{l}8.3 \\
7.1\end{array}$ & $\begin{array}{l}4.1 \\
2.7\end{array}$ & $\begin{array}{r}6.2 \\
12.5\end{array}$ & $\begin{array}{l}11.99 \\
11.90\end{array}$ \\
\hline 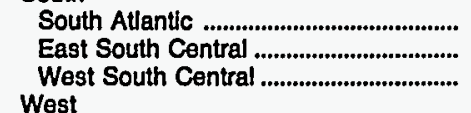 & $\begin{array}{l}46.7 \\
61.8 \\
32.3\end{array}$ & $\begin{array}{l}24.9 \\
31.4 \\
17.4\end{array}$ & $Q_{6.3}^{5.8}$ & $\begin{array}{l}3.4 \\
4.3 \\
3.5\end{array}$ & $\begin{array}{r}12.6 \\
13.6 \\
5.0\end{array}$ & $\begin{array}{l}22.34 \\
22.35 \\
18.59\end{array}$ \\
\hline 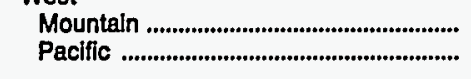 & $\begin{array}{l}63.0 \\
38.7\end{array}$ & $\begin{array}{l}31.1 \\
18.2\end{array}$ & $\begin{array}{r}9.0 \\
10.8\end{array}$ & $Q$ & $\begin{array}{l}4.2 \\
5.6\end{array}$ & $\begin{array}{r}10.42 \\
8.85\end{array}$ \\
\hline 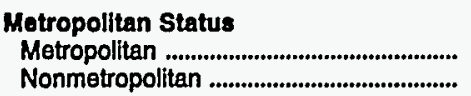 & $\begin{array}{l}46.9 \\
67.7\end{array}$ & $\begin{array}{l}27.4 \\
47.5\end{array}$ & $\begin{array}{l}7.4 \\
9.7\end{array}$ & $\begin{array}{l}5.1 \\
3.2\end{array}$ & $\begin{array}{l}7.0 \\
7.4\end{array}$ & $\begin{array}{l}6.90 \\
9.34\end{array}$ \\
\hline
\end{tabular}

See footnote at end of table. 
Table B8. Energy End-Use Intensities for Natural Gas, 1989 (Continued)

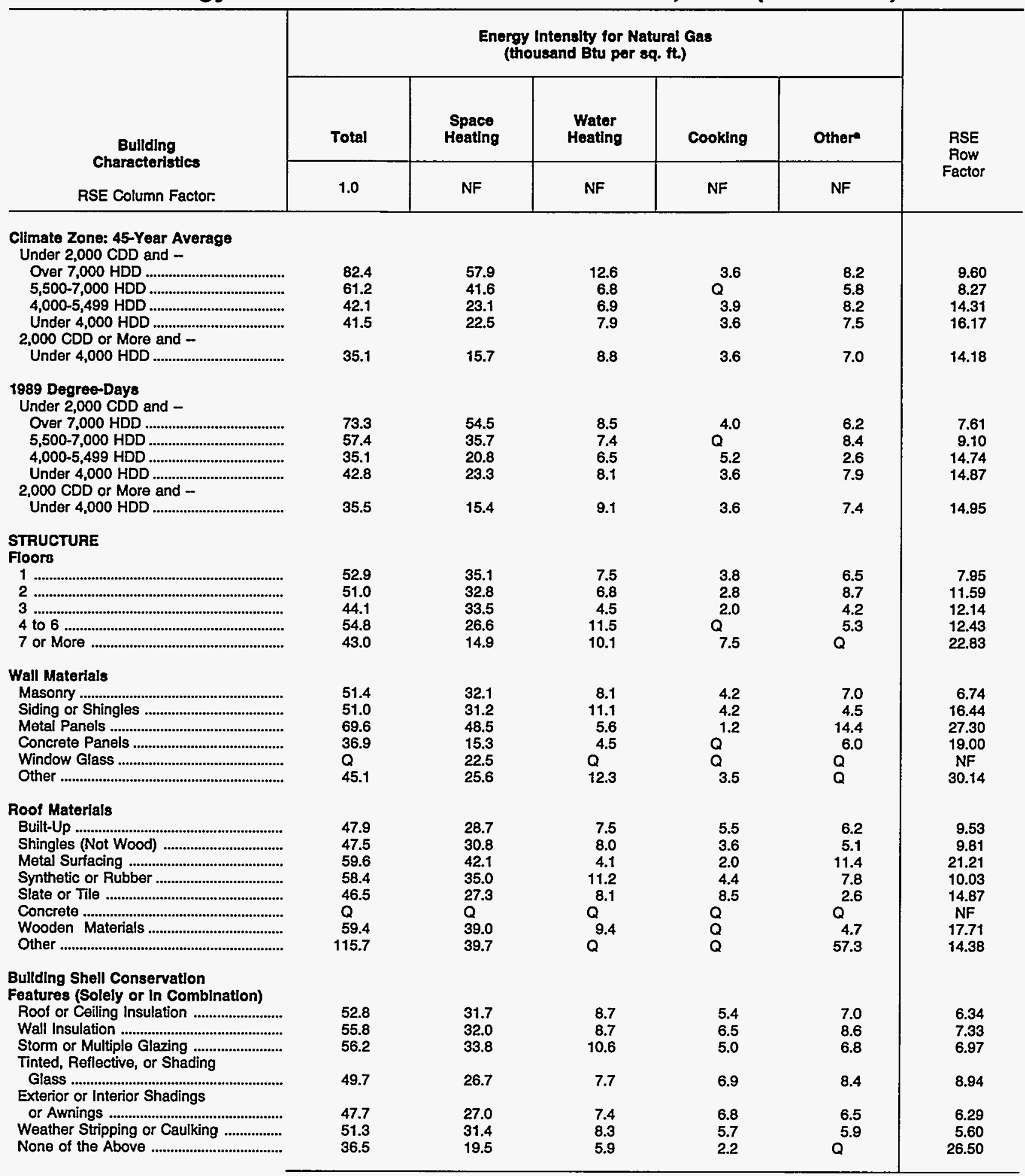

See footnote at end of table. 
Table B8. Energy End-Use Intensities for Natural Gas, 1989 (Continued)

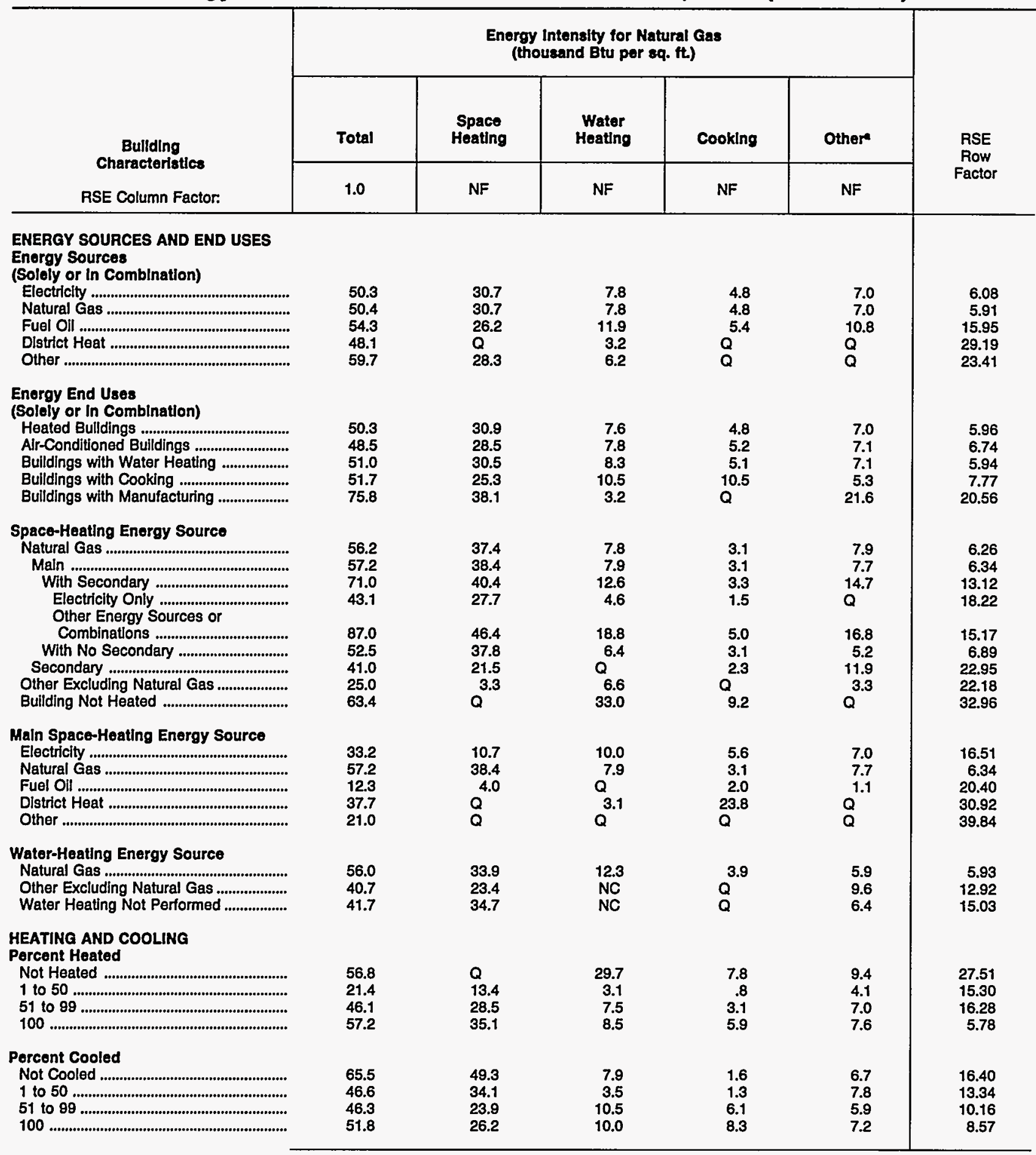

See footnotes at end of table. 
Table B8. Energy End-Use Intensities for Natural Gas, 1989 (Continued)

\begin{tabular}{|c|c|c|c|c|c|c|}
\hline \multirow{3}{*}{$\begin{array}{c}\text { Building } \\
\text { Characteristics } \\
\text { RSE Column Factor: }\end{array}$} & \multicolumn{5}{|c|}{$\begin{array}{l}\text { Energy Intensity for Natural Gas } \\
\text { (thousand Btu per sq. ft.) }\end{array}$} & \multirow{3}{*}{$\begin{array}{l}\text { RSE } \\
\text { Row } \\
\text { Factor }\end{array}$} \\
\hline & \multirow[t]{2}{*}{ Total } & \multirow{2}{*}{$\begin{array}{c}\begin{array}{c}\text { Space } \\
\text { Heating }\end{array} \\
\text { NF }\end{array}$} & \multirow{2}{*}{$\begin{array}{c}\begin{array}{c}\text { Water } \\
\text { Heating }\end{array} \\
\text { NF }\end{array}$} & \multirow{2}{*}{$\frac{\text { Cooking }}{\text { NF }}$} & \multirow{2}{*}{ Other* } & \\
\hline & & & & & & \\
\hline \multicolumn{7}{|l|}{$\begin{array}{l}\text { Heating Equipment } \\
\text { (Solely or in Comblnation) }\end{array}$} \\
\hline 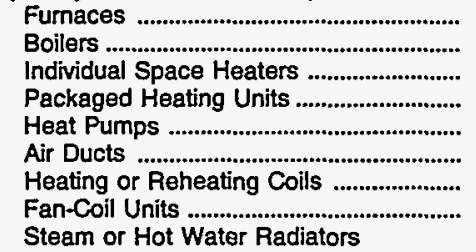 & $\begin{array}{l}54.3 \\
64.0 \\
48.3 \\
49.5 \\
50.6 \\
50.4 \\
55.2 \\
56.9\end{array}$ & $\begin{array}{l}36.3 \\
40.9 \\
27.9 \\
28.1 \\
24.0 \\
28.4 \\
26.6 \\
29.0\end{array}$ & $\begin{array}{r}6.7 \\
10.9 \\
6.3 \\
7.0 \\
8.9 \\
8.1 \\
9.9 \\
10.2\end{array}$ & $\begin{array}{l}3.0 \\
Q^{3.4} \\
4.7 \\
3.9 \\
Q^{5.9} \\
Q\end{array}$ & $\begin{array}{r}8.3 \\
8.8 \\
9.1 \\
9.8 \\
13.7 \\
8.0 \\
10.3 \\
8.3\end{array}$ & $\begin{array}{r}9.16 \\
7.63 \\
10.28 \\
10.39 \\
19.19 \\
7.20 \\
10.94 \\
9.84\end{array}$ \\
\hline 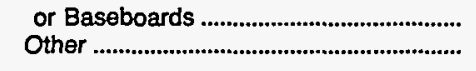 & $\begin{array}{l}62.7 \\
76.5\end{array}$ & $\begin{array}{l}36.6 \\
32.0\end{array}$ & $\begin{array}{l}8.6 \\
8.8\end{array}$ & Q 2.8 & $\mathrm{Q}^{9.4}$ & $\begin{array}{l}11.87 \\
27.31\end{array}$ \\
\hline \multicolumn{7}{|l|}{$\begin{array}{l}\text { Cooling Equipment } \\
\text { (Solely or In Comblnation) }\end{array}$} \\
\hline 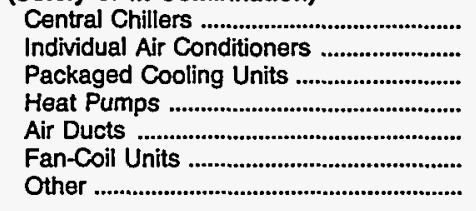 & $\begin{array}{l}51.9 \\
49.6 \\
47.6 \\
52.6 \\
50.4 \\
54.8 \\
71.2\end{array}$ & $\begin{array}{l}22.8 \\
31.4 \\
28.7 \\
25.0 \\
28.2 \\
22.6 \\
11.3\end{array}$ & $\begin{array}{r}10.6 \\
8.4 \\
7.2 \\
7.9 \\
8.6 \\
11.9 \\
Q\end{array}$ & $\begin{array}{r}9.9 \\
3.6 \\
5.9 \\
4.8 \\
6.3 \\
11.8 \\
46.6\end{array}$ & $\begin{array}{r}8.6 \\
6.2 \\
5.8 \\
14.9 \\
7.4 \\
8.6 \\
Q\end{array}$ & $\begin{array}{r}11.23 \\
12.42 \\
8.07 \\
16.86 \\
7.27 \\
11.97 \\
16.81\end{array}$ \\
\hline \multicolumn{7}{|l|}{$\begin{array}{l}\text { ENERGY MANAGENENT } \\
\text { Occupant Control }\end{array}$} \\
\hline 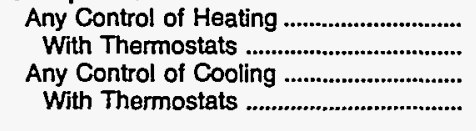 & $\begin{array}{l}46.9 \\
47.2 \\
45.7 \\
45.5\end{array}$ & $\begin{array}{l}28.0 \\
27.7 \\
26.8 \\
26.2\end{array}$ & $\begin{array}{l}8.0 \\
8.2 \\
8.3 \\
8.0\end{array}$ & $\begin{array}{l}4.1 \\
4.0 \\
4.2 \\
4.3\end{array}$ & $\begin{array}{l}6.8 \\
7.2 \\
6.4 \\
6.9\end{array}$ & $\begin{array}{r}9.26 \\
9.69 \\
9.70 \\
10.05\end{array}$ \\
\hline \multicolumn{7}{|l|}{ Reduced Use During Otf-Hours } \\
\hline 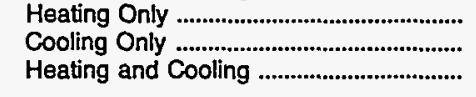 & $\begin{array}{l}63.6 \\
62.5 \\
42.9\end{array}$ & $\begin{array}{l}47.5 \\
30.5 \\
27.1\end{array}$ & $\begin{array}{l}7.3 \\
6.5 \\
5.2\end{array}$ & $Q_{4.5}^{2.3}$ & $Q^{6.6}$ & $\begin{array}{r}14.68 \\
26.27 \\
8.07\end{array}$ \\
\hline \multicolumn{7}{|l|}{$\begin{array}{l}\text { Computerized Energy Management } \\
\text { and Control System }\end{array}$} \\
\hline 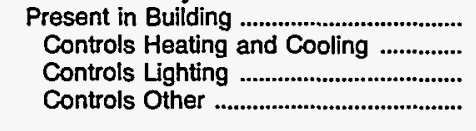 & $\begin{array}{l}46.6 \\
47.3 \\
45.5 \\
62.1\end{array}$ & $\begin{array}{l}22.8 \\
23.4 \\
15.7 \\
25.6\end{array}$ & $\begin{array}{l}7.7 \\
7.6 \\
4.6 \\
8.5\end{array}$ & $\begin{array}{l}\mathrm{Q} \\
\mathrm{Q} \\
\mathrm{Q} \\
3.9\end{array}$ & $\begin{array}{l}8.2 \\
Q^{8.3} \\
24.1\end{array}$ & $\begin{array}{r}10.11 \\
9.93 \\
19.83 \\
25.24\end{array}$ \\
\hline $\begin{array}{l}\text { Other Energy Management } \\
\text { Regular HVAC Maintenance ..................... } \\
\text { Participated in Utility }\end{array}$ & 54.7 & 32.3 & 8.7 & 5.4 & 8.3 & 6.21 \\
\hline Conservation Program .............................. & 55.7 & 32.5 & 10.2 & 5.1 & 8.0 & 12.22 \\
\hline
\end{tabular}

- Includes cooling.

$N C=$ No cases in responding sample.

NF $=$ No applicable RSE row/column factor.

$\mathrm{Q}=$ Data withheld because the Relative Standard Error (RSE) was greater than 50 percent, or data were reported for fewer than 20 buildings.

Notes: - To obtain the RSE percentage for any table cell, multiply the corresponding RSE column and RSE row factors. $\bullet$ See Glossary for explanation of abbreviations and definitions of terms used in this report. Because of rounding, data may not sum to totals.

Source: Energy Information Administration, Office of Energy Markets and End Use, Forms ElA-871A through F of the 1989 Commercial Buildings Energy Consumption Survey. 
Table B9. End-Use Consumption Percentages for Natural Gas, 1989

\begin{tabular}{|c|c|c|c|c|c|}
\hline \multirow[b]{2}{*}{$\begin{array}{l}\text { Bullding } \\
\text { Characteristics }\end{array}$} & \multicolumn{5}{|c|}{ Percent of Natural Gas Consumption } \\
\hline & Total & $\begin{array}{c}\text { Space } \\
\text { Heating }\end{array}$ & $\begin{array}{l}\text { Water } \\
\text { Heating }\end{array}$ & Cooking & Othere \\
\hline 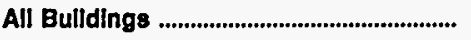 & 100 & 61 & 15 & 10 & 14 \\
\hline 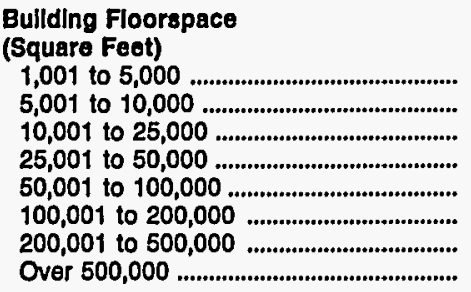 & $\begin{array}{l}100 \\
100 \\
100 \\
100 \\
100 \\
100 \\
100 \\
100\end{array}$ & $\begin{array}{l}61 \\
65 \\
72 \\
67 \\
75 \\
64 \\
48 \\
26\end{array}$ & $\begin{array}{l}20 \\
16 \\
15 \\
10 \\
12 \\
18 \\
19 \\
13\end{array}$ & $\begin{array}{r}12 \\
4 \\
4 \\
5 \\
5 \\
12 \\
7 \\
34\end{array}$ & $\begin{array}{r}8 \\
14 \\
9 \\
18 \\
8 \\
7 \\
26 \\
27\end{array}$ \\
\hline 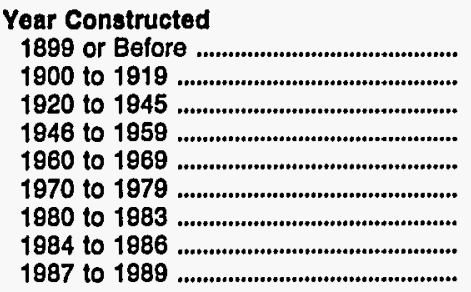 & $\begin{array}{l}100 \\
100 \\
100 \\
100 \\
100 \\
100 \\
100 \\
100 \\
100\end{array}$ & $\begin{array}{l}81 \\
60 \\
64 \\
57 \\
67 \\
59 \\
52 \\
56 \\
59\end{array}$ & $\begin{array}{r}10 \\
10 \\
8 \\
17 \\
14 \\
20 \\
18 \\
21 \\
17\end{array}$ & $\begin{array}{r}4 \\
6 \\
8 \\
Q \\
5 \\
11 \\
9 \\
10 \\
12\end{array}$ & $\begin{array}{l}Q^{4} \\
20 \\
11 \\
14 \\
10 \\
22 \\
14 \\
12\end{array}$ \\
\hline 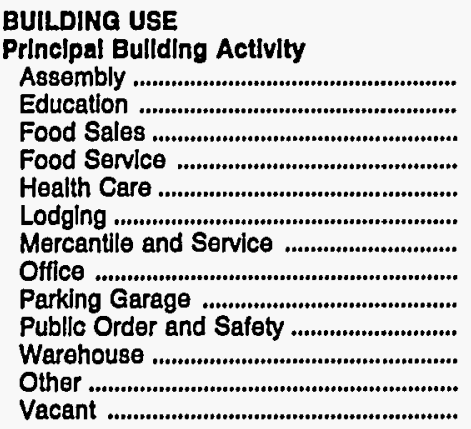 & $\begin{array}{l}100 \\
100 \\
100 \\
100 \\
100 \\
100 \\
100 \\
100 \\
100 \\
100 \\
100 \\
100 \\
100\end{array}$ & $\begin{array}{l}78 \\
73 \\
49 \\
29 \\
24 \\
42 \\
66 \\
72 \\
75 \\
88 \\
76 \\
56 \\
63\end{array}$ & $\begin{array}{r}7 \\
5 \\
3 \\
34 \\
38 \\
45 \\
11 \\
11 \\
Q \\
5 \\
2 \\
6 \\
12\end{array}$ & $\begin{array}{l}10 \\
Q \\
45 \\
32 \\
16 \\
9 \\
5 \\
2 \\
Q \\
Q \\
Q \\
Q \\
Q\end{array}$ & $\begin{array}{c}5 \\
6 \\
2 \\
5 \\
Q \\
5 \\
18 \\
15 \\
Q \\
5 \\
22 \\
34 \\
23\end{array}$ \\
\hline 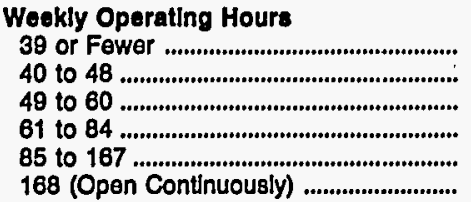 & $\begin{array}{l}100 \\
100 \\
100 \\
100 \\
100 \\
100\end{array}$ & $\begin{array}{l}85 \\
80 \\
75 \\
62 \\
53 \\
40\end{array}$ & $\begin{array}{r}8 \\
7 \\
7 \\
14 \\
12 \\
31\end{array}$ & $\begin{array}{r}2 \\
2 \\
4 \\
10 \\
24 \\
10\end{array}$ & $\begin{array}{r}5 \\
11 \\
14 \\
14 \\
10 \\
19\end{array}$ \\
\hline 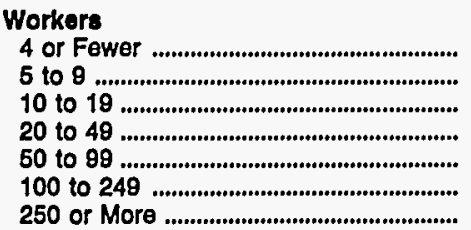 & $\begin{array}{l}100 \\
100 \\
100 \\
100 \\
100 \\
100 \\
100\end{array}$ & $\begin{array}{l}73 \\
67 \\
63 \\
69 \\
71 \\
60 \\
33\end{array}$ & $\begin{array}{r}17 \\
17 \\
16 \\
15 \\
8 \\
11 \\
23\end{array}$ & $\begin{array}{r}4 \\
8 \\
7 \\
6 \\
5 \\
8 \\
24\end{array}$ & $\begin{array}{r}5 \\
8 \\
13 \\
10 \\
16 \\
21 \\
20\end{array}$ \\
\hline
\end{tabular}

See footnotes at end of table. 
Table B9. End-Use Consumption Percentages for Natural Gas, 1989 (Continued)

\begin{tabular}{|c|c|c|c|c|c|}
\hline \multirow[b]{2}{*}{$\begin{array}{l}\text { Bullding } \\
\text { Characteristics }\end{array}$} & \multicolumn{5}{|c|}{ Percent of Natural Gas Consumption } \\
\hline & Total & $\begin{array}{l}\text { Space } \\
\text { Heating }\end{array}$ & $\begin{array}{l}\text { Water } \\
\text { Heating }\end{array}$ & Cooking & Other" \\
\hline \multicolumn{6}{|l|}{ Ownershlp and Occupancy } \\
\hline 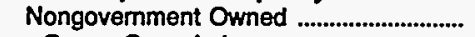 & 100 & 59 & 16 & 8 & 16 \\
\hline 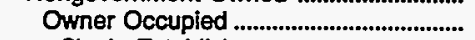 & 100 & 58 & 16 & 8 & 18 \\
\hline Single Establishment .................................. & 100 & 57 & 17 & 8 & 18 \\
\hline Multiple Establishment ........................... & 100 & 61 & 15 & 10 & 15 \\
\hline 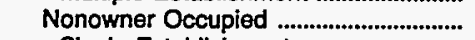 & 100 & 65 & 14 & 9 & 12 \\
\hline 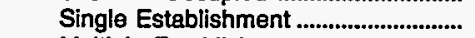 & 100 & 70 & 10 & $\mathbf{9}$ & 11 \\
\hline Multiple Establishment ............................ & 100 & 61 & 20 & 12 & 7 \\
\hline 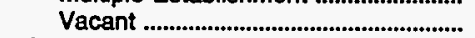 & 100 & 54 & 16 & $\mathbf{Q}$ & 30 \\
\hline 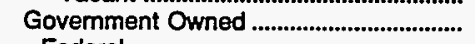 & 100 & 67 & 13 & $\mathbf{Q}$ & 6 \\
\hline 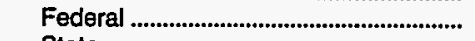 & 100 & 23 & $\mathbf{Q}$ & 68 & $Q$ \\
\hline 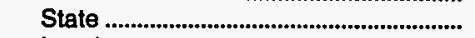 & 100 & 70 & 16 & $\mathbf{Q}$ & 10 \\
\hline 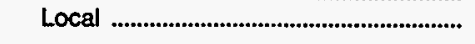 & 100 & 76 & 14 & 6 & 5 \\
\hline \multicolumn{6}{|l|}{ Multibullding Facility } \\
\hline Not on Multibuilding Facility ...................... & 100 & 66 & 15 & 7 & 13 \\
\hline $\begin{array}{l}\text { Part of Multibuilding Facility ................................. } \\
\text { On Facility with Central }\end{array}$ & 100 & 55 & 16 & 13 & 16 \\
\hline 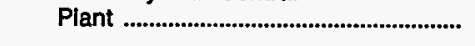 & 100 & 43 & 16 & 20 & 21 \\
\hline \multicolumn{6}{|l|}{$\begin{array}{l}\text { Percent Vacant at Least Three } \\
\text { Months }\end{array}$} \\
\hline 0 & 100 & 63 & 16 & 8 & 13 \\
\hline 1 to 50 & 100 & 55 & 13 & 9 & 23 \\
\hline 51 to 99 & 100 & 45 & 5 & 47 & 4 \\
\hline 100 & 100 & 64 & 20 & 1 & 15 \\
\hline \multicolumn{6}{|l|}{ Months in Use Out of Past 12 Months } \\
\hline 0 to 8 & 100 & 59 & 23 & 13 & 6 \\
\hline 9 to 11 & 100 & 77 & 11 & 1 & 11 \\
\hline 12 & 100 & 60 & 15 & 10 & 15 \\
\hline \multicolumn{6}{|l|}{$\begin{array}{l}\text { LOCATION } \\
\text { Census Region }\end{array}$} \\
\hline 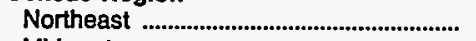 & 100 & 66 & 15 & 9 & 10 \\
\hline 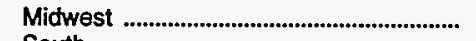 & 100 & 70 & 12 & 6 & 13 \\
\hline 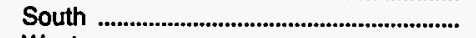 & 100 & 53 & 17 & $\mathbf{9}$ & 22 \\
\hline 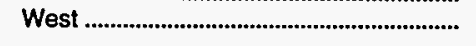 & 100 & 48 & 21 & 20 & 11 \\
\hline \multirow{2}{*}{\multicolumn{6}{|c|}{$\begin{array}{l}\text { Census Divlsion } \\
\text { Northeast }\end{array}$}} \\
\hline & & & & & \\
\hline 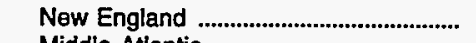 & 100 & 49 & 30 & 9 & 11 \\
\hline & 13 & 8 & 10 \\
\hline 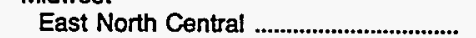 & 100 & 71 & 13 & & 10 \\
\hline 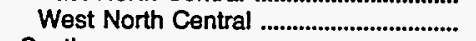 & 100 & 67 & 11 & 4 & $\begin{array}{l}10 \\
19\end{array}$ \\
\hline \multicolumn{6}{|l|}{ South } \\
\hline 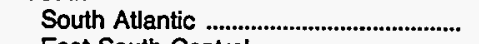 & 100 & 53 & 12 & 7 & 27 \\
\hline 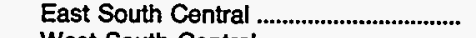 & 100 & 51 & 20 & 7 & 22 \\
\hline 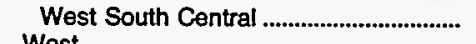 & 100 & 54 & 19 & 11 & 16 \\
\hline \multicolumn{6}{|l|}{ West } \\
\hline 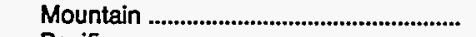 & 100 & 49 & 14 & Q & 7 \\
\hline 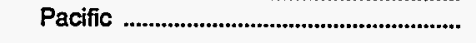 & 100 & 47 & 28 & 11 & 14 \\
\hline \multicolumn{6}{|l|}{ Metropolltan Status } \\
\hline Metropolitan & 100 & 58 & 16 & 11 & 15 \\
\hline 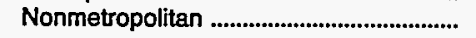 & 100 & 70 & 14 & 5 & 11 \\
\hline
\end{tabular}

See footnotes at end of table. 


\begin{tabular}{|c|c|c|c|c|c|}
\hline \multirow[b]{2}{*}{$\begin{array}{l}\text { Bullding } \\
\text { Characteristics }\end{array}$} & \multicolumn{5}{|c|}{ Percent of Natural Gas Consumption } \\
\hline & Total & $\begin{array}{l}\text { Space } \\
\text { Heating }\end{array}$ & $\begin{array}{l}\text { Water } \\
\text { Heating }\end{array}$ & Cooking & Othere \\
\hline \multicolumn{6}{|l|}{$\begin{array}{l}\text { Cllmate Zone: 45-Year Average } \\
\text { Under 2,000 CDD and - }\end{array}$} \\
\hline 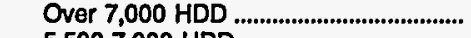 & 100 & 70 & 15 & 4 & 10 \\
\hline 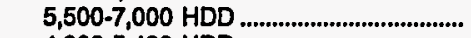 & 100 & 68 & 11 & 11 & 9 \\
\hline 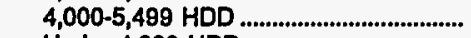 & 100 & 55 & 16 & 9 & 19 \\
\hline $\begin{array}{l}\text { Under 4,000 HDD ...................................... } \\
2,000 \text { CDD or More and - }\end{array}$ & 100 & 54 & 19 & 9 & 18 \\
\hline 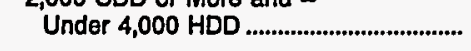 & 100 & 45 & 25 & 10 & 20 \\
\hline \multicolumn{6}{|l|}{$\begin{array}{l}1989 \text { Degree-Days } \\
\text { Under } 2,000 \text { CDD and -- }\end{array}$} \\
\hline Over 7,000 HDD .............................................. & 100 & 74 & 12 & 5 & 9 \\
\hline 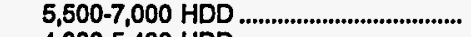 & 100 & 62 & 13 & 10 & 15 \\
\hline 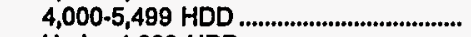 & 100 & 59 & 19 & 15 & 7 \\
\hline $\begin{array}{l}\text { Under 4,000 HDD ........................................... } \\
\text { 2,000 CDD or More and - }\end{array}$ & 100 & 54 & 19 & 8 & 18 \\
\hline 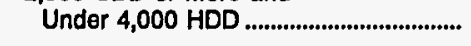 & 100 & 43 & 26 & 10 & 21 \\
\hline \multicolumn{6}{|l|}{$\begin{array}{l}\text { STRUCTURE } \\
\text { Floors }\end{array}$} \\
\hline 1 & 100 & 66 & 14 & 7 & 12 \\
\hline 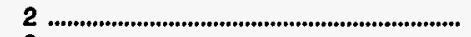 & 100 & 64 & 13 & 5 & 17 \\
\hline (1) & 100 & 76 & 10 & 4 & 9 \\
\hline 4 to 6 & 100 & 49 & 21 & $Q$ & 10 \\
\hline 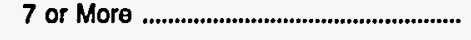 & 100 & 35 & 23 & 17 & 25 \\
\hline \multicolumn{6}{|l|}{ Wall Materials } \\
\hline Masonry & 100 & 62 & 16 & 8 & 14 \\
\hline Siding or Shingles ............................................... & 100 & 61 & 22 & 8 & 9 \\
\hline 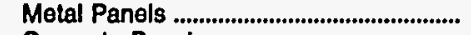 & 100 & 70 & 8 & 2 & 21 \\
\hline 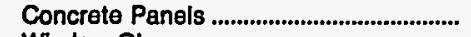 & 100 & 41 & 12 & 30 & 16 \\
\hline Window Glass ......................................................... & 100 & 58 & $\mathbf{Q}$ & 13 & 8 \\
\hline 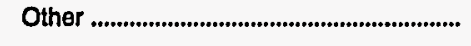 & 100 & 57 & 27 & $Q$ & $Q$ \\
\hline \multicolumn{6}{|l|}{ Roof Materlals } \\
\hline 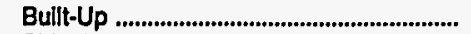 & 100 & 60 & 16 & 12 & 13 \\
\hline 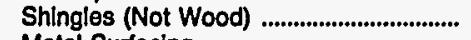 & 100 & 65 & 17 & 8 & 11 \\
\hline Metal Surfacing ................................................... & 100 & 71 & 7 & 3 & 19 \\
\hline 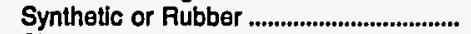 & 100 & 60 & 19 & 8 & 13 \\
\hline 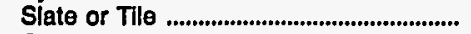 & 100 & 59 & 17 & 18 & 6 \\
\hline 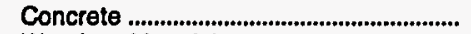 & 100 & 53 & 37 & 5 & 4 \\
\hline Wooden Materials ........................................... & 100 & 66 & 16 & $\mathbf{Q}$ & 8 \\
\hline Other & 100 & 34 & $\mathbf{Q}$ & $\mathbf{Q}$ & 49 \\
\hline \multicolumn{6}{|l|}{$\begin{array}{l}\text { Bullding Shell Conservation } \\
\text { Features (Solely or In Combination) }\end{array}$} \\
\hline Roof or Celling Insulation ........................... & 100 & 60 & 16 & 10 & 13 \\
\hline 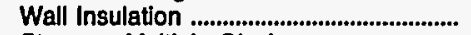 & 100 & 57 & 16 & 12 & 15 \\
\hline $\begin{array}{l}\text { Storm or Multiple Glazing .............................. } \\
\text { Tinted, Reflective, or Shading }\end{array}$ & 100 & 60 & 19 & 9 & 12 \\
\hline 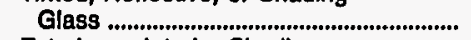 & 100 & 54 & 15 & 14 & 17 \\
\hline Exterior or Interior Shadings & & & & & \\
\hline 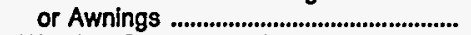 & 100 & 57 & 16 & 14 & 14 \\
\hline Weather Stripping or Caulking ................. & 100 & 61 & 16 & 11 & 11 \\
\hline None of the Above & 100 & 53 & 16 & 6 & Q \\
\hline
\end{tabular}

See footnotes at end of table. 


\begin{tabular}{|c|c|c|c|c|c|}
\hline \multirow[b]{2}{*}{$\begin{array}{c}\text { Bullding } \\
\text { Characteristics }\end{array}$} & \multicolumn{5}{|c|}{ Percent of Natural Gas Consumption } \\
\hline & Total & $\begin{array}{c}\text { Space } \\
\text { Heating }\end{array}$ & $\begin{array}{l}\text { Water } \\
\text { Heating }\end{array}$ & Cooking & Other \\
\hline
\end{tabular}

\section{ENERGY SOURCES AND END USES}

Energy Sources

(Solely or In Combination)

Electricity .

Fuol Oil

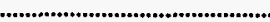

Other .

100

100

100

100

100

\section{Energy End Uses}

(Solely or In Comblnation)

Heated Buildings

Air-Conditioned Buildings

Buildings with Water Heating ...................

Buildings with Cooking ...

Buildings with Menufacturing

Space-Heating Energy Source

Natural Gas

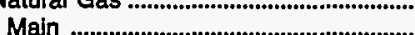

With Secondary

Electricity Only

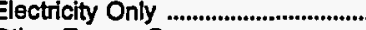

Other Energy Sources or

Combinations .....

With No Secondary

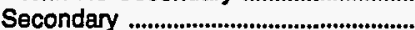

Other Excluding Natural Gas .....................

Building Not Heated

\section{Main Space-Heating Energy Source}

Electricity .

Natural Gas

Fuel Oil

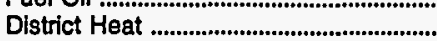

Other

Water-Heating Energy Source

Natural Gas

Other Excluding Natural Ges

Water Heating Not Performed

\section{HEATING AND COOLING}

Percent Heated

Not Heated

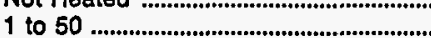

51 to 99

100

Percent Cooled

Not Cooled

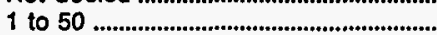

51 to 99

100 .

Heating Equipment

Heating Equipment
(Solely or In Combination)

Furnaces

Builers ........................................................

Individual Space Heaters ..........................

Packaged Heating Units

Heat Pumps

Air Ducts

Heating or Reheating Coils ....................

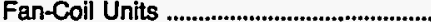

Steam or Hot Water Radiators

or Baseboards .

Other

See footnotes at end of table.

100

100

100

100

100

100

100

100

100

100

100

100

100

100

100

100

100

100

100

100

100

100

100

100

100

100

100
100

100

100

100

100
100
100

100

100

100

100

100

100

100
61

61

48

25
47

15
15
22
7
10

10
10
10
42
$Q$

14

26

14
58

83

61

59

60

49
50

67

67

53

72
52

13

32

67

32

15
16
16
20
4

$\begin{array}{rr}9 & 14 \\ 11 & 15 \\ 10 & 14 \\ 20 & 10 \\ Q & 28\end{array}$

14

14
14
18

18

22

22

$\mathbf{Q}$

26

52

30

14

44

Q

22

NC

$\begin{array}{rl}6 & 14 \\ 6 & 13 \\ 5 & 21 \\ 3 & 0 \\ & \\ 6 & 19 \\ 6 & 10 \\ 6 & 29 \\ 47 & 13 \\ 14 & 17\end{array}$

17
6
16
63
0

21

13

9

10

60

83

17

63

62

52

15

16

7

$\mathrm{Q}$

1

24
15

75
73
52
51

12

7

23

$\begin{array}{rr}14 & 17 \\ 4 & 19 \\ 7 & 15 \\ 10 & 13\end{array}$

$\begin{array}{rr}2 & 10 \\ 3 & 17 \\ 13 & 13 \\ 16 & 14\end{array}$

14
7
10
9
9
10

(1)


Table B9. End-Use Consumption Percentages for Natural Gas, 1989 (Continued)

\begin{tabular}{|c|c|c|c|c|c|}
\hline \multirow[b]{2}{*}{$\begin{array}{c}\text { Bullding } \\
\text { Characteristics }\end{array}$} & \multicolumn{5}{|c|}{ Percent of Natural Gas Consumption } \\
\hline & Total & $\begin{array}{l}\text { Space } \\
\text { Heating }\end{array}$ & $\begin{array}{l}\text { Water } \\
\text { Heating }\end{array}$ & Cooking & Other" \\
\hline 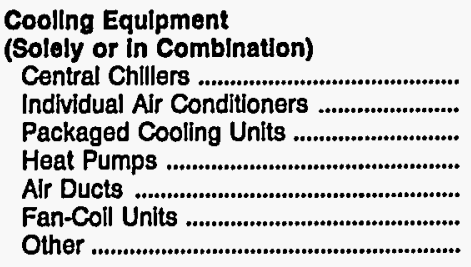 & $\begin{array}{l}100 \\
100 \\
100 \\
100 \\
100 \\
100 \\
100\end{array}$ & $\begin{array}{l}44 \\
63 \\
60 \\
48 \\
56 \\
41 \\
16\end{array}$ & $\begin{array}{l}20 \\
17 \\
15 \\
15 \\
17 \\
22 \\
Q\end{array}$ & $\begin{array}{r}19 \\
7 \\
12 \\
9 \\
12 \\
21 \\
65\end{array}$ & $\begin{array}{l}17 \\
12 \\
12 \\
28 \\
15 \\
16 \\
Q\end{array}$ \\
\hline 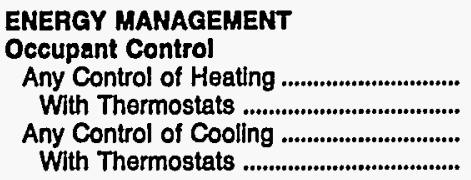 & $\begin{array}{l}100 \\
100 \\
100 \\
100\end{array}$ & $\begin{array}{l}60 \\
59 \\
59 \\
58\end{array}$ & $\begin{array}{l}17 \\
17 \\
18 \\
18\end{array}$ & $\begin{array}{l}9 \\
8 \\
9 \\
9\end{array}$ & $\begin{array}{l}14 \\
15 \\
14 \\
15\end{array}$ \\
\hline 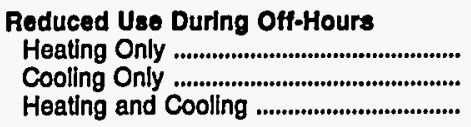 & $\begin{array}{l}100 \\
100 \\
100\end{array}$ & $\begin{array}{l}75 \\
49 \\
63\end{array}$ & $\begin{array}{l}11 \\
10 \\
12\end{array}$ & $\begin{array}{r}4 \\
11 \\
11\end{array}$ & $\begin{array}{l}10 \\
30 \\
14\end{array}$ \\
\hline 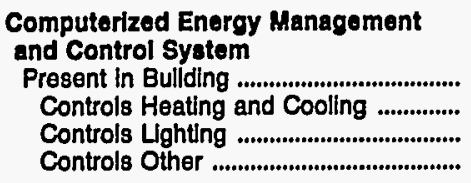 & $\begin{array}{l}100 \\
100 \\
100 \\
100\end{array}$ & $\begin{array}{l}49 \\
49 \\
35 \\
41\end{array}$ & $\begin{array}{l}16 \\
16 \\
10 \\
14\end{array}$ & $\begin{array}{r}17 \\
17 \\
41 \\
6\end{array}$ & $\begin{array}{l}18 \\
18 \\
14 \\
39\end{array}$ \\
\hline $\begin{array}{l}\text { Other Energy Management } \\
\text { Regular HVAC Malntenance ..................... } \\
\text { Particlpated in Utillty } \\
\text { Conservation Program }\end{array}$ & $\begin{array}{l}100 \\
100\end{array}$ & $\begin{array}{l}59 \\
58\end{array}$ & $\begin{array}{l}16 \\
18\end{array}$ & 10 & 15 \\
\hline
\end{tabular}

- Includes cooling.

- = Value rounds to zero In the units displayed.

NC $=$ No cases in responding sample.

$Q=$ Data withheld because the Relative Standard Error (RSE) was greater than 50 percent, or data were reported for fewer than 20 buildings.

Notes: - See Glossary for explanation of abbreviations and definitions of terms used in this report. - Because of rounding, data may not sum to totals.

Source: Energy Information Administration, Office of Energy Markets and End Use, Forms ElA-871A through F of the 1989 Commercial Bulldings Energy Consumption Survey. 


\begin{tabular}{|c|c|c|c|c|c|}
\hline \multirow{3}{*}{$\begin{array}{c}\text { Building } \\
\text { Characteristics } \\
\text { RSE Column Factor: }\end{array}$} & \multicolumn{4}{|c|}{$\begin{array}{l}\text { Fuel Oll Consumption } \\
\text { (trillion Btu) }\end{array}$} & \multirow{3}{*}{$\begin{array}{c}\text { RSE } \\
\text { Row } \\
\text { Factor }\end{array}$} \\
\hline & Total & $\begin{array}{l}\text { Space } \\
\text { Heating }\end{array}$ & $\begin{array}{l}\text { Water } \\
\text { Heating }\end{array}$ & Othere & \\
\hline & 1.0 & NF & NF & NF & \\
\hline 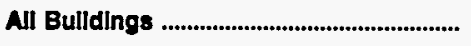 & 357 & 301 & 26 & 30 & 13.28 \\
\hline $\begin{array}{l}\text { Bullding Foorspace } \\
\text { (Square Foet) } \\
1,001 \text { to } 10,000 \\
10,001 \text { to } 25,000 \\
25,001 \text { to } 50,000 \ldots \ldots \ldots \ldots \\
50,001 \text { to } 100,000 \\
100,001 \text { to } 200,000 \\
\text { Over } 200,000\end{array}$ & $\begin{array}{r}101 \\
69 \\
47 \\
54 \\
46 \\
40\end{array}$ & $\begin{array}{l}91 \\
61 \\
44 \\
47 \\
31 \\
27\end{array}$ & $\begin{array}{l}9 \\
Q^{2} \\
Q^{2} \\
Q\end{array}$ & $\begin{array}{l}\mathbf{Q} \\
\mathbf{Q} \\
\mathbf{Q} \\
\mathbf{Q} \\
\mathbf{Q} \\
\mathbf{Q}\end{array}$ & $\begin{array}{l}23.83 \\
17.35 \\
19.69 \\
46.65 \\
39.53 \\
29.27\end{array}$ \\
\hline $\begin{array}{l}\text { Year Constructed } \\
1945 \text { or Before } \\
1946 \text { to } 1959 \\
1960 \text { to } 1969 \\
1970 \text { to } 1979 \\
1980 \text { to } 1989\end{array}$ & $\begin{array}{r}112 \\
77 \\
73 \\
61 \\
34\end{array}$ & $\begin{array}{r}101 \\
73 \\
60 \\
40 \\
27\end{array}$ & $\begin{array}{r}8 \\
3 \\
6 \\
6 \\
\end{array}$ & $\begin{array}{l}\mathbf{Q} \\
\mathbf{Q} \\
\mathbf{Q} \\
\mathbf{Q} \\
\mathbf{Q}\end{array}$ & $\begin{array}{l}13.63 \\
24.41 \\
36.42 \\
30.29 \\
33.98\end{array}$ \\
\hline 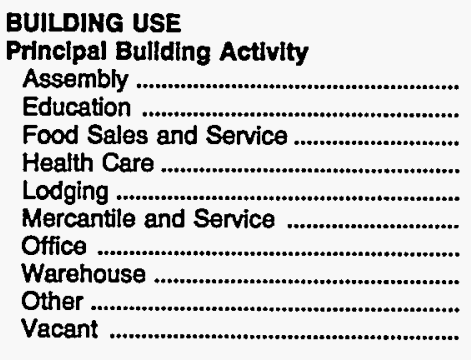 & $\begin{array}{r}31 \\
71 \\
18 \\
17 \\
10 \\
75 \\
43 \\
53 \\
30 \\
7\end{array}$ & $\begin{array}{r}29 \\
68 \\
15 \\
7 \\
6 \\
68 \\
38 \\
39 \\
24 \\
7\end{array}$ & $\begin{array}{ll} & \\
Q^{2} & \\
Q & \\
Q & \\
& 4 \\
& 4 \\
& 2 \\
Q^{2} & \\
Q & \\
Q & \end{array}$ & $\begin{array}{l}Q \\
Q \\
Q \\
Q \\
Q \\
Q \\
Q \\
Q \\
Q \\
Q\end{array}$ & $\begin{array}{l}20.25 \\
24.77 \\
28.86 \\
42.28 \\
33.61 \\
22.11 \\
27.63 \\
38.96 \\
44.52 \\
34.88\end{array}$ \\
\hline 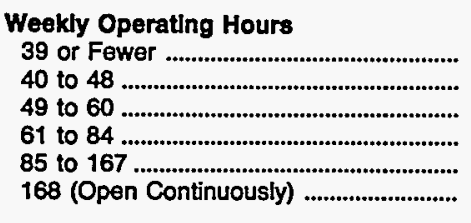 & $\begin{array}{l}26 \\
65 \\
54 \\
68 \\
80 \\
65\end{array}$ & $\begin{array}{l}25 \\
63 \\
49 \\
61 \\
67 \\
37\end{array}$ & $Q_{11} \begin{array}{r}1 \\
3 \\
6\end{array}$ & $\begin{array}{l}Q \\
Q \\
Q \\
Q \\
Q \\
Q\end{array}$ & $\begin{array}{l}23.63 \\
19.31 \\
18.95 \\
20.54 \\
32.60 \\
30.29\end{array}$ \\
\hline $\begin{array}{l}\text { Workers } \\
4 \text { or Feiver } \\
5 \text { to } 9 \\
10 \text { to } 19 \\
20 \text { to } 49 \\
50 \text { to } 99 \\
100 \text { to } 249 \\
250 \text { or More }\end{array}$ & $\begin{array}{l}83 \\
35 \\
38 \\
72 \\
34 \\
57 \\
39\end{array}$ & $\begin{array}{l}75 \\
29 \\
37 \\
66 \\
30 \\
42 \\
21\end{array}$ & $\begin{array}{l}7 \\
3 \\
\\
1 \\
\\
Q^{4} \\
Q^{4} \\
Q\end{array}$ & $\begin{array}{l}Q \\
Q \\
Q \\
Q \\
Q \\
Q \\
Q \\
Q \\
Q\end{array}$ & $\begin{array}{l}19.56 \\
21.87 \\
25.85 \\
17.57 \\
27.13 \\
42.47 \\
35.13\end{array}$ \\
\hline 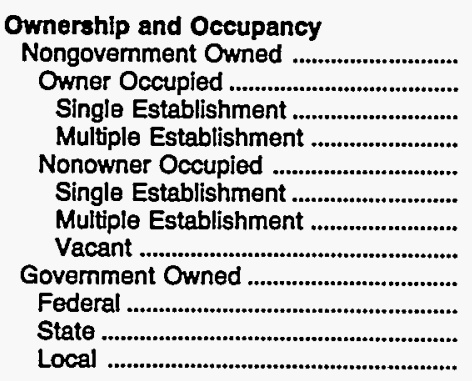 & $\begin{array}{r}242 \\
200 \\
176 \\
24 \\
42 \\
Q^{19} \\
Q^{115} \\
Q^{31} \\
79\end{array}$ & $\begin{array}{r}197 \\
165 \\
144 \\
21 \\
32 \\
11 \\
17 \\
Q^{103} \\
Q^{10} \\
25 \\
75\end{array}$ & $\begin{array}{r}19 \\
17 \\
15 \\
1 \\
2 \\
Q^{2} \\
Q^{2} \\
Q^{2} \\
Q^{3} \\
Q_{3}\end{array}$ & $\begin{array}{l}Q \\
Q \\
Q \\
Q \\
Q \\
Q \\
Q \\
N C \\
Q \\
Q \\
Q \\
Q\end{array}$ & $\begin{array}{c}14.28 \\
17.06 \\
18.40 \\
15.14 \\
29.37 \\
\text { NF } \\
29.73 \\
\text { NF } \\
22.24 \\
\text { NF } \\
42.68 \\
23.52\end{array}$ \\
\hline
\end{tabular}

See footnotes at end of table. 
Table B10. Consumption of Fuel Oil by End Use, 1989 (Continued)

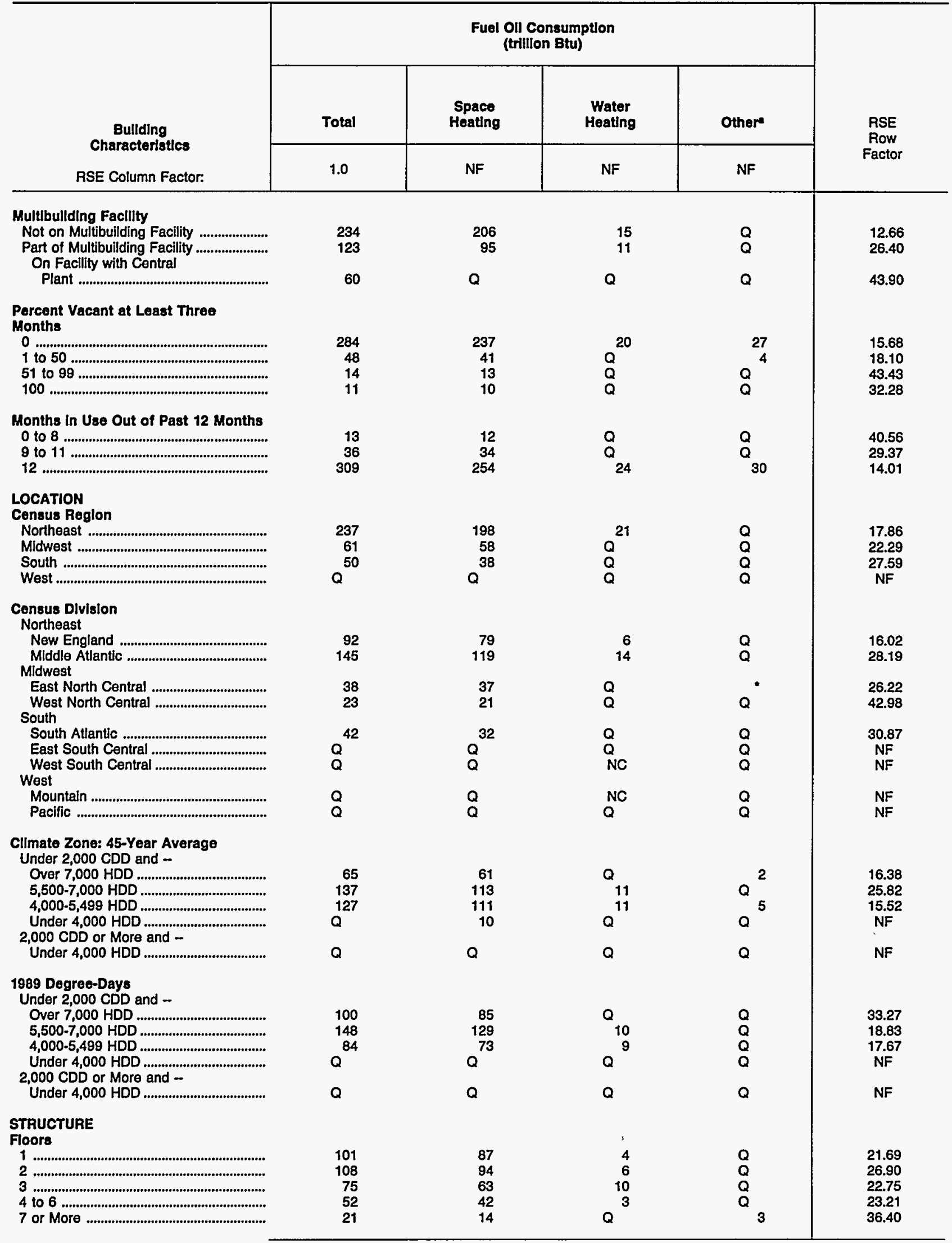

See footnotes at end of table. 


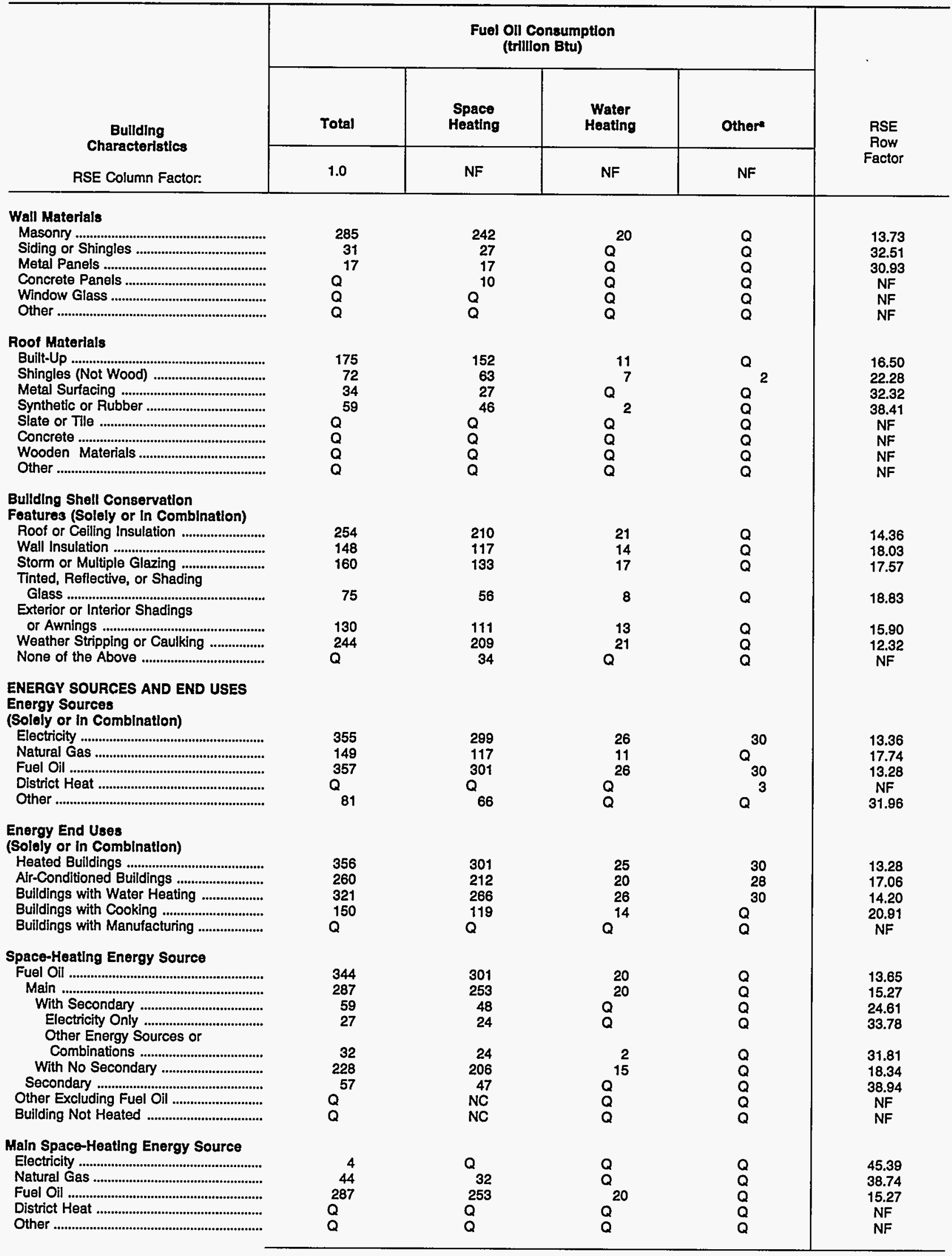

See footnotes at end of table. 
Table B10. Consumption of Fuel Oil by End Use, 1989 (Continued)

\begin{tabular}{|c|c|c|c|c|c|}
\hline \multirow{3}{*}{$\begin{array}{c}\begin{array}{c}\text { Building } \\
\text { Characteristics }\end{array} \\
\text { RSE Column Factor: }\end{array}$} & \multicolumn{4}{|c|}{$\begin{array}{l}\text { Fuel Oll Consumption } \\
\text { (trillion Btu) }\end{array}$} & \multirow{3}{*}{$\begin{array}{l}\text { RSE } \\
\text { Row } \\
\text { Factor }\end{array}$} \\
\hline & Total & $\begin{array}{l}\text { Space } \\
\text { Heating }\end{array}$ & $\begin{array}{l}\text { Water } \\
\text { Heating }\end{array}$ & Other" & \\
\hline & 1.0 & NF & NF & NF & \\
\hline 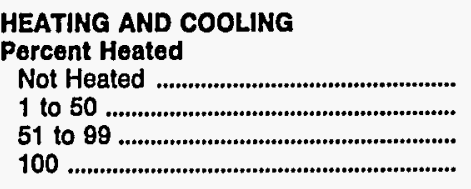 & $\begin{array}{r}Q \\
27 \\
62 \\
264\end{array}$ & $\begin{array}{r}Q \\
25 \\
45 \\
228\end{array}$ & $\begin{array}{l}\mathbf{Q} \\
\mathbf{Q} \\
22\end{array}$ & $\begin{array}{l}\mathbf{Q} \\
\mathbf{Q} \\
\mathbf{Q} \\
\mathbf{Q}\end{array}$ & $\begin{array}{l}\text { NF } \\
26.51 \\
35.05 \\
12.05\end{array}$ \\
\hline $\begin{array}{l}\text { Percent Cooled } \\
\text { Not Cooled } \\
1 \text { to } 50 \\
51 \text { to } 99 . \ldots \ldots \ldots \\
100\end{array}$ & $\begin{array}{r}97 \\
146 \\
65 \\
50\end{array}$ & $\begin{array}{r}89 \\
119 \\
52 \\
40\end{array}$ & $\begin{array}{l}6 \\
9 \\
6 \\
5\end{array}$ & $\begin{array}{l}Q^{2} \\
Q \\
Q\end{array}$ & $\begin{array}{l}10.68 \\
23.47 \\
26.17 \\
23.02\end{array}$ \\
\hline 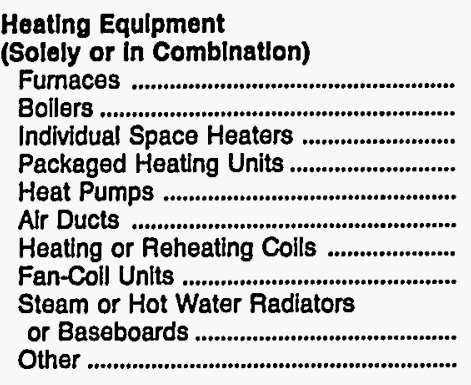 & $\begin{array}{r}99 \\
282 \\
113 \\
28 \\
16 \\
181 \\
89 \\
78 \\
\\
216 \\
Q\end{array}$ & $\begin{array}{r}85 \\
235 \\
89 \\
18 \\
12 \\
143 \\
63 \\
60 \\
\\
174 \\
Q\end{array}$ & $\begin{array}{l}5^{5} \\
Q^{7} \\
Q^{11} \\
Q^{11} \\
Q^{18}\end{array}$ & $\begin{array}{l}Q_{27} \\
Q^{27} \\
Q^{3} \\
Q^{27} \\
Q^{27} \\
Q^{24}\end{array}$ & $\begin{array}{c}21.30 \\
14.83 \\
26.89 \\
43.40 \\
30.08 \\
21.00 \\
32.68 \\
19.99 \\
\\
17.38 \\
\text { NF }\end{array}$ \\
\hline 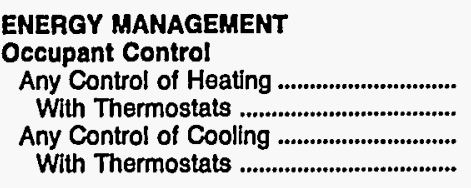 & $\begin{array}{l}157 \\
143 \\
134 \\
104\end{array}$ & $\begin{array}{r}137 \\
125 \\
116 \\
89\end{array}$ & $\begin{array}{r}10 \\
9 \\
10 \\
8\end{array}$ & $\begin{array}{l}Q \\
Q \\
Q \\
Q\end{array}$ & $\begin{array}{l}12.55 \\
13.87 \\
17.02 \\
17.67\end{array}$ \\
\hline 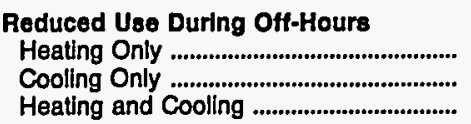 & $\begin{array}{r}83 \\
24 \\
172\end{array}$ & $\begin{array}{r}78 \\
20 \\
143\end{array}$ & $\begin{array}{r}4 \\
3 \\
14\end{array}$ & $\begin{array}{l}\mathbf{Q} \\
\mathbf{Q} \\
\mathbf{Q}\end{array}$ & $\begin{array}{l}12.39 \\
33.78 \\
16.04\end{array}$ \\
\hline 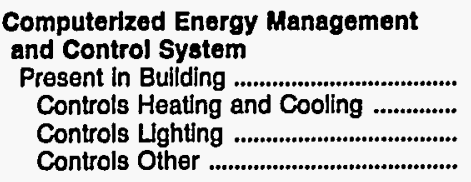 & $\begin{array}{l}51 \\
\mathbf{5 0} \\
Q^{\mathbf{5}}\end{array}$ & $\mathrm{Q}^{31}$ & $\begin{array}{l}Q \\
Q \\
Q \\
Q\end{array}$ & $\begin{array}{l}Q \\
Q \\
Q \\
Q\end{array}$ & $\begin{array}{l}27.14 \\
27.24 \\
\text { NF } \\
\text { NF }\end{array}$ \\
\hline 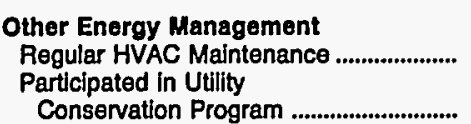 & $\begin{array}{r}283 \\
64\end{array}$ & $\begin{array}{r}235 \\
53\end{array}$ & $\begin{array}{r}20 \\
2\end{array}$ & $\begin{array}{r}28 \\
Q^{28}\end{array}$ & $\begin{array}{l}14.16 \\
25.62\end{array}$ \\
\hline
\end{tabular}

- Includes cookling and cooling.

- = Value rounds to zero in the units displayed.

$N C=$ No ceses in responding sample.

NF $=$ No applicable RSE row/column factor.

$Q=$ Data withheld because the Relative Standard Error (RSE) was greater than 50 percent, or data were reported for fewer than 20 buildings.

Notes: - To obtain the RSE percentage for any table cell, multiply the corresponding RSE column and RSE row factors. - See Glossary for explanation of abbreviations and definitions of terms used in this report. Because of rounding, data may not sum to totals.

Source: Energy Information Administration, Office of Energy Markets and End Use, Forms EIA-871A through F of the 1989 Commercial Buildings Energy Consumption Survey. 
Table B11. Energy End-Use Intensities for Fuel Oil, 1989

\begin{tabular}{|c|c|c|c|c|c|}
\hline \multirow{3}{*}{$\begin{array}{c}\text { Bullding } \\
\text { Characteristics } \\
\text { RSE Column Factor: }\end{array}$} & \multicolumn{4}{|c|}{$\begin{array}{l}\text { Energy Intensity for Fuel Oll } \\
\text { (thousand Btu per sq. ft) }\end{array}$} & \multirow{3}{*}{$\begin{array}{c}\text { RSE } \\
\text { Row } \\
\text { Factor. }\end{array}$} \\
\hline & Total & $\begin{array}{l}\text { Space } \\
\text { Heating }\end{array}$ & $\begin{array}{l}\text { Water } \\
\text { Heating }\end{array}$ & Other & \\
\hline & 1.0 & NF & NF & NF & \\
\hline 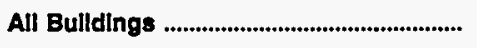 & 28.3 & 23.9 & 2.0 & 2.4 & 11.84 \\
\hline 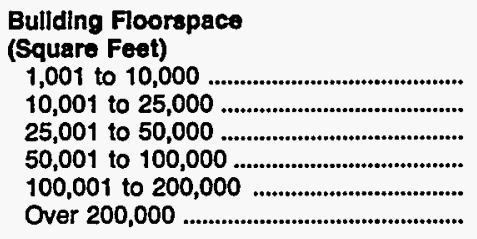 & $\begin{array}{r}66.1 \\
45.9 \\
35.5 \\
32.5 \\
20.7 \\
9.2\end{array}$ & $\begin{array}{r}59.9 \\
40.8 \\
33.0 \\
27.8 \\
13.9 \\
6.3\end{array}$ & $\begin{array}{l}5.6 \\
3.6 \\
Q \\
Q \\
Q \\
Q\end{array}$ & $\begin{array}{l}\mathbf{Q} \\
\mathbf{Q} \\
\mathbf{Q} \\
\mathbf{Q} \\
\mathbf{Q} \\
\mathbf{Q}\end{array}$ & $\begin{array}{l}14.22 \\
13.54 \\
16.34 \\
47.93 \\
35.67 \\
26.37\end{array}$ \\
\hline 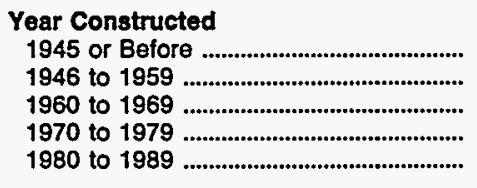 & $\begin{array}{l}30.4 \\
37.3 \\
32.0 \\
25.4 \\
15.7\end{array}$ & $\begin{array}{l}27.4 \\
35.3 \\
26.2 \\
16.9 \\
12.4\end{array}$ & $\begin{array}{l}2.1 \\
1.5 \\
2.4 \\
2.5 \\
Q\end{array}$ & $\begin{array}{l}\mathbf{Q} \\
\mathbf{Q} \\
\mathbf{Q} \\
\mathbf{Q} \\
\mathbf{Q}\end{array}$ & $\begin{array}{l}11.98 \\
16.67 \\
39.13 \\
29.64 \\
30.76\end{array}$ \\
\hline 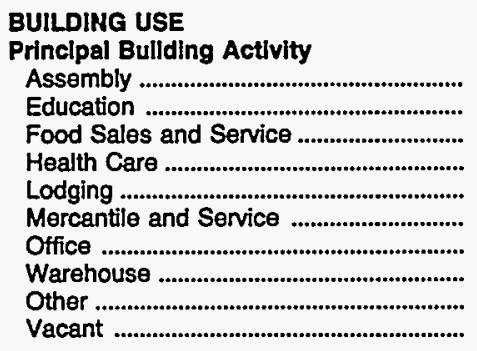 & $\begin{array}{l}29.4 \\
32.1 \\
Q \\
12.2 \\
17.4 \\
46.7 \\
14.8 \\
37.3 \\
41.3 \\
22.3\end{array}$ & $\begin{array}{l}27.2 \\
30.8 \\
43.2 \\
5.1 \\
Q \\
42.1 \\
13.1 \\
27.2 \\
32.6 \\
21.7\end{array}$ & $\begin{array}{l}2.0 \\
1.1 \\
Q^{1.1} \\
Q \\
7.1 \\
2.7 \\
{ }^{.8} \\
Q \\
Q \\
Q\end{array}$ & 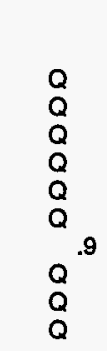 & $\begin{array}{c}16.41 \\
22.62 \\
\text { NF } \\
40.09 \\
44.71 \\
16.22 \\
27.57 \\
39.10 \\
38.51 \\
40.94\end{array}$ \\
\hline 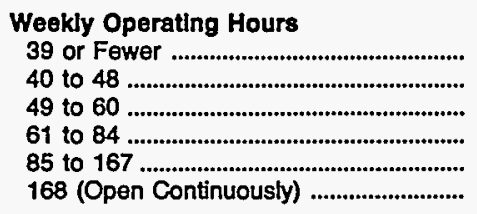 & $\begin{array}{l}33.7 \\
24.5 \\
22.8 \\
38.0 \\
36.1 \\
22.9\end{array}$ & $\begin{array}{l}32.6 \\
23.6 \\
20.8 \\
34.1 \\
30.3 \\
12.9\end{array}$ & $\begin{array}{r}1.1 \\
.5 \\
1.2 \\
3.4 \\
Q \\
3.7\end{array}$ & $\begin{array}{l}Q \\
Q \\
Q \\
Q \\
Q \\
Q \\
Q\end{array}$ & $\begin{array}{l}13.51 \\
25.32 \\
17.77 \\
17.97 \\
21.88 \\
28.19\end{array}$ \\
\hline $\begin{array}{l}\text { Workers } \\
4 \text { or Fower } \\
5 \text { to } 9 \\
10 \text { to } 19 \\
20 \text { to } 49 \\
50 \text { to } 99 \\
100 \text { to } 249 \\
250 \text { or More }\end{array}$ & $\begin{array}{l}47.9 \\
31.9 \\
42.9 \\
47.8 \\
18.6 \\
33.7 \\
10.0\end{array}$ & $\begin{array}{r}43.3 \\
27.1 \\
41.5 \\
44.2 \\
16.6 \\
24.6 \\
5.5\end{array}$ & $\begin{array}{l}4.0 \\
3.2 \\
1.3 \\
2.9 \\
\mathbf{Q} \\
\mathbf{Q} \\
\mathbf{Q}\end{array}$ & $\begin{array}{l}Q \\
Q \\
Q \\
Q \\
Q \\
Q \\
Q \\
Q\end{array}$ & $\begin{array}{l}14.17 \\
16.14 \\
22.75 \\
16.52 \\
35.92 \\
38.58 \\
31.74\end{array}$ \\
\hline 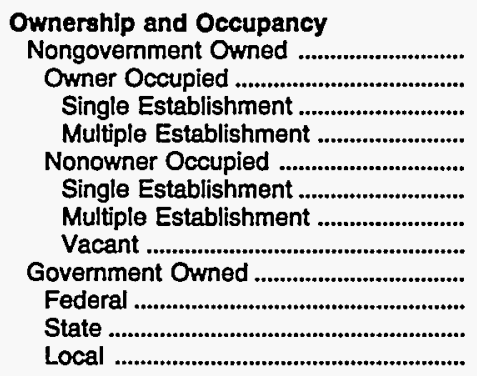 & $\begin{array}{l}27.6 \\
28.8 \\
31.7 \\
17.5 \\
22.9 \\
17.5 \\
30.8 \\
33.9 \\
29.9 \\
Q \\
32.5 \\
31.6\end{array}$ & $\begin{array}{l}22.5 \\
23.8 \\
25.9 \\
15.3 \\
17.5 \\
10.1 \\
28.0 \\
33.1 \\
26.9 \\
Q \\
Q \\
30.0\end{array}$ & $\begin{array}{l}2.1 \\
2.4 \\
2.8 \\
.9 \\
1.2 \\
Q \\
2.6 \\
Q \\
Q \\
Q \\
Q \\
1.2\end{array}$ & $\begin{array}{l}Q \\
\mathbf{Q} \\
\mathbf{Q} \\
\mathbf{Q} \\
\mathbf{Q} \\
\mathbf{Q} \\
\mathbf{Q} \\
\mathrm{NC} \\
\mathbf{Q} \\
\mathbf{Q} \\
\mathbf{Q} \\
\mathbf{Q}\end{array}$ & $\begin{array}{l}12.91 \\
14.00 \\
15.84 \\
14.94 \\
28.05 \\
47.37 \\
17.28 \\
40.46 \\
19.04 \\
\text { NF } \\
25.35 \\
22.93\end{array}$ \\
\hline
\end{tabular}

See footnote at end of table. 
Table B11. Energy End-Use Intensities for Fuel Oll, 1989 (Continued)

\begin{tabular}{|c|c|c|c|c|c|}
\hline \multirow{3}{*}{$\begin{array}{c}\text { Bullding } \\
\text { Characteristlcs } \\
\text { RSE Column Factor: }\end{array}$} & \multicolumn{4}{|c|}{$\begin{array}{l}\text { Energy Intensity for Fuel Oll } \\
\text { (thousand Btu per sq. tt.) }\end{array}$} & \multirow{3}{*}{$\begin{array}{c}\text { RSE } \\
\text { Row } \\
\text { Factor }\end{array}$} \\
\hline & Total & $\begin{array}{l}\text { Space } \\
\text { Heating }\end{array}$ & $\begin{array}{l}\text { Water } \\
\text { Heating }\end{array}$ & Other" & \\
\hline & 1.0 & NF & NF & NF & \\
\hline \multicolumn{6}{|l|}{ Multlbullding Facillty } \\
\hline Not on Multibuilding Facility ......................... & 30.0 & 26.4 & 1.9 & $\mathbf{Q}$ & 11.27 \\
\hline $\begin{array}{l}\text { Part of Multibuilding Facility .......................... } \\
\text { On Facillty with Central }\end{array}$ & 25.6 & 19.7 & 2.3 & $\overline{\mathbf{Q}}$ & 25.11 \\
\hline 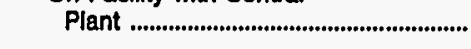 & 26.2 & $\mathbf{Q}$ & $\mathbf{Q}$ & $\mathbf{Q}$ & 43.16 \\
\hline \multicolumn{6}{|l|}{$\begin{array}{l}\text { Percent Vacant at Least Three } \\
\text { Months }\end{array}$} \\
\hline 0 & 31.7 & 26.4 & 2.3 & 3.0 & 13.89 \\
\hline 1 to 50 & 19.3 & 16.5 & $\mathbf{Q}$ & 1.4 & 16.96 \\
\hline 51 to 99 & $\begin{array}{l}19.6 \\
25.4\end{array}$ & $\begin{array}{l}17.4 \\
24.8\end{array}$ & $0^{2.1}$ & $\mathbf{Q}$ & 32.38 \\
\hline \multicolumn{6}{|l|}{ Months In Use Out of Past 12 Months } \\
\hline 0 to 8 . & $\begin{array}{l}23.2 \\
40.1\end{array}$ & 22.2 & $Q_{Q}^{Q}$ & $Q^{Q}$ & $\begin{array}{l}26.40 \\
33.23\end{array}$ \\
\hline 12 & 27.6 & 22.8 & 2.2 & 2.7 & 12.73 \\
\hline \multicolumn{6}{|l|}{$\begin{array}{l}\text { LOCATION } \\
\text { Census Reglon }\end{array}$} \\
\hline 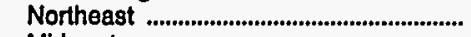 & 46.1 & 38.6 & 4.0 & 3.5 & 13.01 \\
\hline Mldwest ...................................................................... & 19.0 & 18.3 & $\mathbf{Q}$ & Q & 26.27 \\
\hline South & 17.7 & 13.5 & $Q$ & $Q$ & 32.63 \\
\hline West & $\mathbf{Q}$ & $\mathbf{Q}$ & $\mathbf{Q}$ & $\mathbf{Q}$ & NF \\
\hline \multirow{2}{*}{\multicolumn{6}{|c|}{$\begin{array}{l}\text { Cenaus Divislon } \\
\text { Northeast }\end{array}$}} \\
\hline & & & & & \\
\hline 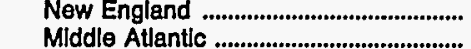 & 48.5 & 41.6 & 3.4 & $\mathbf{Q}$ & 11.05 \\
\hline \multicolumn{6}{|l|}{ 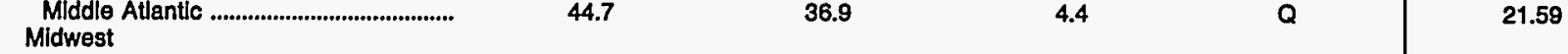 } \\
\hline 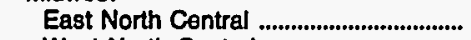 & 19.5 & 19.0 & $\mathbf{Q}$ & $\mathbf{Q}$ & 33.26 \\
\hline 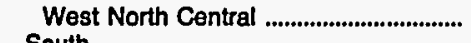 & 18.2 & $\mathbf{Q}$ & $\vec{Q}$ & $\vec{Q}$ & 48.24 \\
\hline \multicolumn{6}{|l|}{ South } \\
\hline South Atlantic ................................................ & 20.8 & 15.5 & $\mathbf{Q}$ & $\mathbf{Q}$ & 39.11 \\
\hline $\begin{array}{l}\text { East South Central } \\
\text { West South Central }\end{array}$ & 16.5 & Q & $\mathbf{Q}$ & $\mathbf{Q}$ & 49.69 \\
\hline 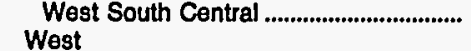 & $\mathbf{Q}$ & $Q$ & NC & $\mathbf{Q}$ & NF \\
\hline \multicolumn{6}{|l|}{ West } \\
\hline 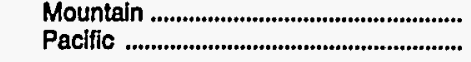 & $\begin{array}{l}\mathbf{Q} \\
\mathbf{Q}\end{array}$ & $\begin{array}{l}\mathbf{Q} \\
\mathbf{Q}\end{array}$ & $\stackrel{N}{Q}^{N C}$ & $\begin{array}{l}\mathbf{Q} \\
\mathbf{Q}\end{array}$ & $\begin{array}{l}\mathrm{NF} \\
\mathrm{NF}\end{array}$ \\
\hline \multirow{2}{*}{\multicolumn{6}{|c|}{$\begin{array}{l}\text { Cllmate Zone: } 45-Y e a r \text { Average } \\
\text { Under 2,000 CDD and - }\end{array}$}} \\
\hline & & & & & \\
\hline 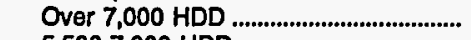 & 35.0 & 32.6 & $Q$ & 1.1 & 15.35 \\
\hline 5,500-7,000 HDD ............................................ & 34.9 & 28.8 & 2.7 & $\mathbf{Q}$ & 23.26 \\
\hline 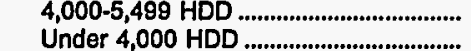 & 29.8 & 25.9 & 2.7 & 1.3 & 10.81 \\
\hline 2,000 CDD or More and - & & & & & \\
\hline Under 4,000 HDD ........................................ & 10.6 & $Q$ & Q & $\mathbf{Q}$ & 49.99 \\
\hline \multicolumn{6}{|l|}{$\begin{array}{l}1989 \text { Degree-Days } \\
\text { Under 2,000 CDD and - }\end{array}$} \\
\hline Over $7,000 \mathrm{HDD}$ & 39.0 & 33.0 & Q & $\mathbf{Q}$ & 28.27 \\
\hline 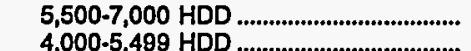 & 28.8 & 25.2 & 1.9 & $\mathbf{Q}$ & 14.97 \\
\hline 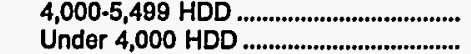 & 32.3 & 28.1 & 3.4 & $\mathbf{Q}$ & 17.07 \\
\hline $2,000 \mathrm{CDD}$ or More and - & & & & $Q$ & $\mathbf{N F}$ \\
\hline 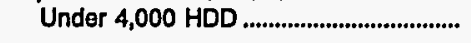 & $\mathbf{Q}$ & Q & $\mathbf{Q}$ & $\mathbf{Q}$ & NF \\
\hline \multicolumn{6}{|l|}{$\begin{array}{l}\text { STRUCTURE } \\
\text { Floors }\end{array}$} \\
\hline $1, \ldots$ & 44.4 & 38.4 & 1.6 & $\mathbf{Q}$ & 18.11 \\
\hline 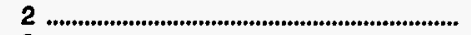 & 35.3 & 30.7 & 1.8 & $\overline{0}$ & 25.45 \\
\hline 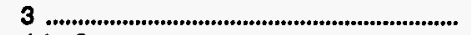 & 36.7 & 30.9 & 4.8 & $\bar{Q}$ & 20.50 \\
\hline 4 to 6 & 19.3 & 15.7 & 1.0 & $\hat{Q}$ & 21.14 \\
\hline 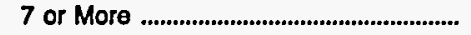 & 8.3 & 5.4 & $\mathbf{Q}$ & 1.2 & 36.46 \\
\hline
\end{tabular}

See footnote at end of table. 
Table B11. Energy End-Use Intensities for Fuel Oil, 1989 (Continued)

\begin{tabular}{|c|c|c|c|c|c|}
\hline \multirow{3}{*}{$\begin{array}{c}\text { Bullding } \\
\text { Characteriatics } \\
\text { RSE Column Factor: }\end{array}$} & \multicolumn{4}{|c|}{$\begin{array}{l}\text { Energy Intensity for Fuel Oll } \\
\text { (thousand Btu per } 8 q . \mathrm{tt} \text { ) }\end{array}$} & \multirow{3}{*}{$\begin{array}{l}\text { RSE } \\
\text { Row } \\
\text { Factor }\end{array}$} \\
\hline & \multirow{2}{*}{$\begin{array}{c}\text { Total } \\
1.0\end{array}$} & \multirow{2}{*}{$\begin{array}{c}\begin{array}{c}\text { Space } \\
\text { Heating }\end{array} \\
\text { NF }\end{array}$} & \multirow{2}{*}{$\begin{array}{c}\begin{array}{c}\text { Water } \\
\text { Heating }\end{array} \\
\text { NF }\end{array}$} & \multirow{2}{*}{ Other" } & \\
\hline & & & & & \\
\hline 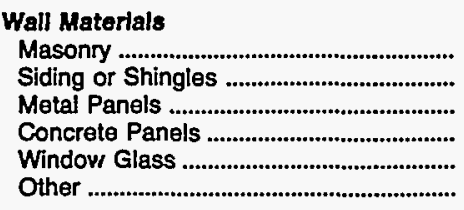 & $\begin{array}{l}30.4 \\
43.9 \\
26.0 \\
13.0 \\
13.9 \\
Q\end{array}$ & $\begin{array}{l}25.8 \\
37.9 \\
25.0 \\
7.8 \\
Q \\
Q\end{array}$ & $\begin{array}{l}2.1 \\
Q \\
Q \\
Q \\
Q \\
Q\end{array}$ & $\begin{array}{l}\mathbf{Q} \\
\mathbf{Q} \\
\mathbf{Q} \\
\mathbf{Q} \\
\mathbf{Q} \\
\mathbf{Q}\end{array}$ & $\begin{array}{l}11.59 \\
24.19 \\
21.54 \\
46.24 \\
45.23 \\
\mathrm{NF}\end{array}$ \\
\hline 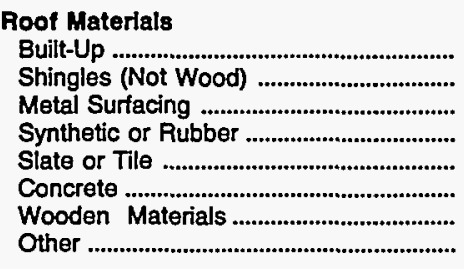 & $\begin{array}{l}26.7 \\
33.7 \\
41.0 \\
33.2 \\
Q \\
7.3 \\
Q \\
Q\end{array}$ & $\begin{array}{l}23.1 \\
29.5 \\
32.8 \\
25.7 \\
Q \\
5.1 \\
Q \\
Q\end{array}$ & $\begin{array}{l}1.7 \\
3.1 \\
Q \\
Q^{1.2} \\
Q \\
Q \\
Q\end{array}$ & $\begin{array}{l}Q \\
Q^{1.1} \\
Q \\
Q \\
Q \\
Q \\
Q\end{array}$ & $\begin{array}{l}14.49 \\
19.54 \\
15.56 \\
37.48 \\
\text { NF } \\
36.98 \\
\text { NF } \\
\text { NF }\end{array}$ \\
\hline 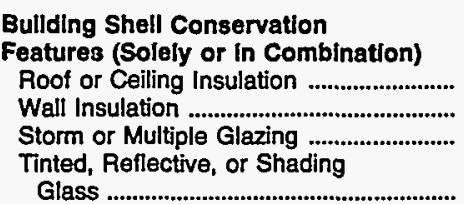 & $\begin{array}{l}26.5 \\
23.2 \\
23.9 \\
15.6\end{array}$ & $\begin{array}{l}21.9 \\
18.3 \\
19.7 \\
11.6\end{array}$ & $\begin{array}{l}2.2 \\
2.1 \\
2.5 \\
1.7\end{array}$ & $\begin{array}{l}Q \\
Q \\
Q \\
Q\end{array}$ & $\begin{array}{l}11.81 \\
15.56 \\
14.28 \\
18.14\end{array}$ \\
\hline 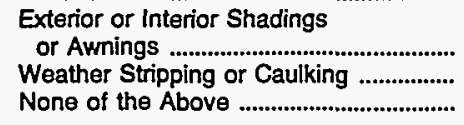 & $\begin{array}{l}22.6 \\
25.9 \\
Q\end{array}$ & $\begin{array}{l}19.2 \\
22.2 \\
29.5\end{array}$ & $Q^{2.2}$ & $\begin{array}{l}Q \\
Q \\
Q\end{array}$ & $\begin{array}{c}13.38 \\
10.91 \\
\mathrm{NF}\end{array}$ \\
\hline 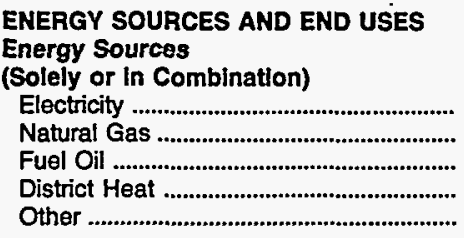 & $\begin{array}{l}28.2 \\
18.9 \\
28.3 \\
Q \\
35.8\end{array}$ & $\begin{array}{l}23.8 \\
14.9 \\
23.9 \\
Q \\
29.2\end{array}$ & $\begin{array}{l}2.1 \\
1.4 \\
2.0 \\
0 \\
2.5\end{array}$ & $\begin{array}{l}Q \\
Q \\
2.4 \\
Q \\
Q\end{array}$ & $\begin{array}{c}12.46 \\
14.78 \\
11.84 \\
\mathrm{NF} \\
19.32\end{array}$ \\
\hline 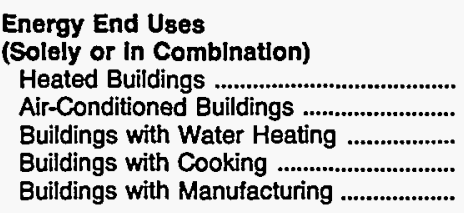 & $\begin{array}{l}28.4 \\
24.8 \\
27.1 \\
24.1 \\
Q\end{array}$ & $\begin{array}{l}24.0 \\
20.2 \\
22.5 \\
19.0 \\
Q\end{array}$ & $\begin{array}{l}2.0 \\
1.9 \\
2.2 \\
2.3 \\
Q\end{array}$ & $\begin{array}{l}2.4 \\
2.7 \\
2.5 \\
Q \\
Q\end{array}$ & $\begin{array}{l}11.87 \\
15.79 \\
12.43 \\
20.01 \\
\mathrm{NF}\end{array}$ \\
\hline 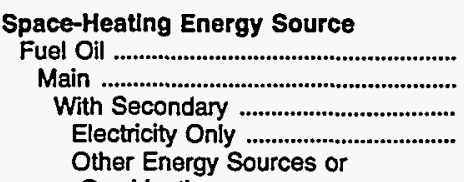 & $\begin{array}{l}32.7 \\
51.3 \\
51.5 \\
57.6\end{array}$ & $\begin{array}{l}28.6 \\
45.3 \\
41.7 \\
51.8\end{array}$ & $\begin{array}{r}1.9 \\
3.5 \\
4.2\end{array}$ & $\begin{array}{l}Q \\
Q \\
Q \\
Q\end{array}$ & $\begin{array}{l}12.21 \\
11.80 \\
16.14 \\
23.48\end{array}$ \\
\hline 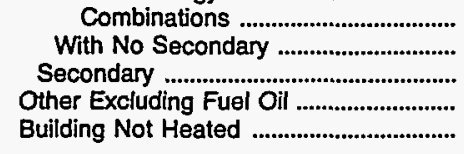 & $\begin{array}{l}47.4 \\
51.3 \\
11.5 \\
Q \\
14.6\end{array}$ & $\begin{array}{r}34.9 \\
46.2 \\
9.6 \\
\text { NC } \\
\text { NC }\end{array}$ & $\begin{array}{l}3.5 \\
3.3 \\
Q \\
Q \\
10.8\end{array}$ & $\begin{array}{l}Q \\
Q \\
Q \\
Q \\
Q\end{array}$ & $\begin{array}{c}18.81 \\
13.83 \\
32.89 \\
N F \\
11.75\end{array}$ \\
\hline 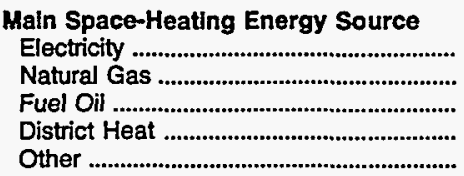 & $\begin{array}{l}3.8 \\
9.3 \\
51.3 \\
Q \\
61.6\end{array}$ & $\begin{array}{r}1.5 \\
6.7 \\
45.3 \\
Q \\
59.5\end{array}$ & $\begin{array}{l}Q \\
Q \\
3.5 \\
Q \\
Q\end{array}$ & $\begin{array}{l}Q \\
Q \\
Q \\
Q \\
Q\end{array}$ & $\begin{array}{c}45.19 \\
35.62 \\
11.80 \\
\mathrm{NF} \\
12.98\end{array}$ \\
\hline
\end{tabular}

See footnote at end of table. 
Table B11. Energy End-Use Intensities for Fuel Oil, 1989 (Continued)

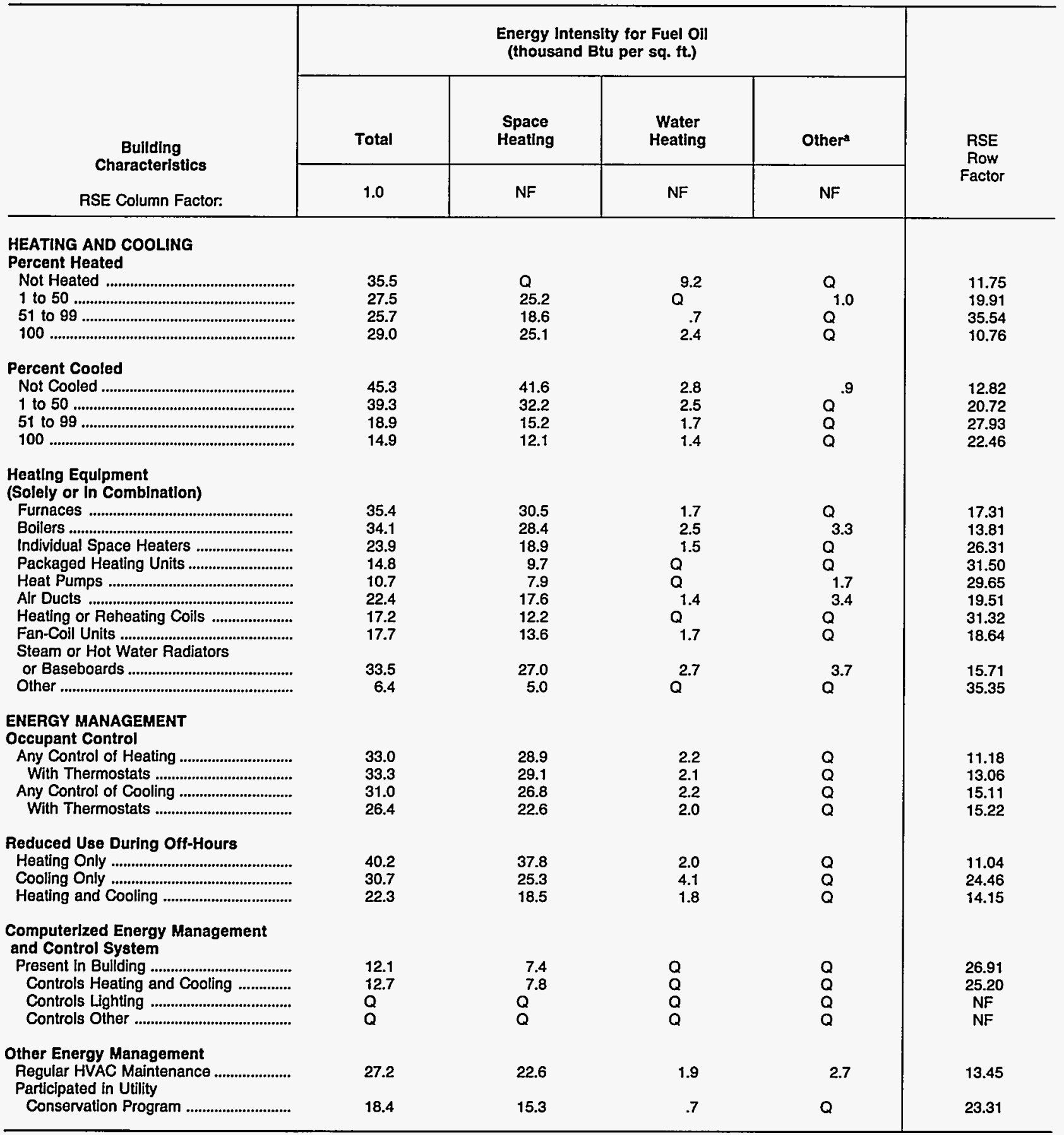

- Includes cooking and cooling.

NC $=$ No cases in responding sample.

$\mathrm{NF}=$ No applicable RSE row/column factor.

$Q=$ Data withheld because the Relative Standard Error (RSE) was greater than 50 percent, or data were reported for fewer than 20 buildings.

Notes: - To obtain the RSE percentage for any table cell, multiply the corresponding RSE column and RSE row factors. - See Glossary for explanation of abbreviations and definitions of terms used in this report. - Because of rounding, data may not sum to totals.

Source: Energy Information Administration, Office of Energy Markets and End Use, Forms EIA-871A through F of the 1989 Commercial Buildings Energy Consumption Survey. 
Table B12. Consumption of District Heat by End Use, 1989

\begin{tabular}{|c|c|c|c|c|c|}
\hline \multirow{3}{*}{$\begin{array}{c}\text { Building } \\
\text { Characteristics } \\
\text { RSE Column Factor: }\end{array}$} & \multicolumn{4}{|c|}{$\begin{array}{l}\text { District Heat Consumption } \\
\text { (trillion Btu) }\end{array}$} & \multirow{3}{*}{$\begin{array}{l}\text { RSE } \\
\text { Row } \\
\text { Factor }\end{array}$} \\
\hline & Total & $\begin{array}{l}\text { Space } \\
\text { Heating }\end{array}$ & $\begin{array}{l}\text { Water } \\
\text { Heating }\end{array}$ & Other" & \\
\hline & 1.0 & NF & $\mathrm{NF}$ & NF & \\
\hline All Buildings & 585 & 355 & 129 & 101 & 21.30 \\
\hline $\begin{array}{l}\text { Bullding Floorspace } \\
\text { (Square Feet) } \\
1,001 \text { to } 10,000 \\
10,001 \text { to } 25,000 \\
25,001 \text { to } 50,000 \\
50,001 \text { to } 100,000 \\
100,001 \text { to } 200,000 \\
\text { Over } 200,000\end{array}$ & $\begin{array}{l}Q \\
Q^{63} \\
Q_{119} \\
106 \\
209\end{array}$ & $\begin{array}{l}Q^{42} \\
Q^{58} \\
71 \\
120\end{array}$ & $\begin{array}{l}\mathbf{Q} \\
\mathbf{Q} \\
\mathbf{Q} \\
\mathbf{Q} \\
\mathbf{Q} \\
\mathbf{Q}\end{array}$ & $\begin{array}{l}Q \\
Q \\
Q_{25} \\
Q_{31}\end{array}$ & $\begin{array}{c}N F \\
44.51 \\
N F \\
49.81 \\
38.40 \\
36.55\end{array}$ \\
\hline $\begin{array}{l}\text { Year Constructed } \\
1945 \text { or Before } \\
1946 \text { to } 1959 \\
1960 \text { to } 1969 \\
1970 \text { to } 1979 \\
1980 \text { to } 1989\end{array}$ & $\begin{array}{l}161 \\
Q^{156} \\
110 \\
Q\end{array}$ & $\begin{array}{l}Q^{96} \\
86 \\
67 \\
24\end{array}$ & $Q^{15}$ & $\begin{array}{l}Q^{20} \\
26 \\
Q^{28}\end{array}$ & $\begin{array}{c}42.01 \\
\mathrm{NF} \\
39.95 \\
33.59 \\
\mathrm{NF}\end{array}$ \\
\hline 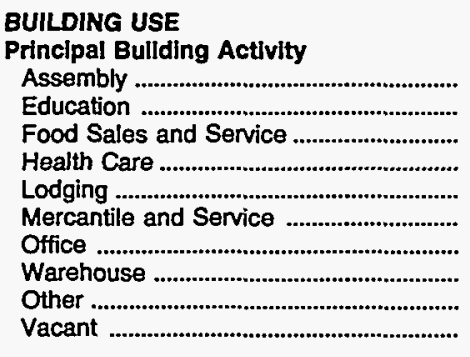 & $\begin{array}{l}Q^{49} \\
Q_{92} \\
Q^{92} \\
6 \\
{ }^{167} \\
Q^{49} \\
Q^{49}\end{array}$ & $\begin{array}{l}Q^{44} \\
Q^{23} \\
Q^{23} \\
Q^{127} \\
Q^{33} \\
Q^{33}\end{array}$ & $\begin{array}{l}Q \\
Q \\
Q \\
Q \\
Q \\
Q \\
{ }^{15} \\
Q \\
Q \\
Q\end{array}$ & $\begin{array}{l}Q \\
Q \\
Q \\
Q \\
Q \\
Q \\
Q \\
{ }^{26} \\
Q^{26} \\
Q \\
Q\end{array}$ & $\begin{array}{c}35.38 \\
N F \\
N F \\
48.56 \\
N F \\
46.28 \\
22.80 \\
N F \\
41.02 \\
N F\end{array}$ \\
\hline $\begin{array}{l}\text { Weekly Operating Hours } \\
39 \text { or Fewer } \\
40 \text { to } 48 \\
49 \text { to } 60 \\
61 \text { to } 84 \\
85 \text { to } 167 \\
168 \text { (Open Continuously) }\end{array}$ & $\begin{array}{l}Q \\
105 \\
66 \\
60 \\
Q \\
272\end{array}$ & $\begin{array}{l}Q_{79} \\
55 \\
39 \\
Q^{124}\end{array}$ & $\stackrel{Q}{Q}_{Q_{90}^{Q}}^{Q}$ & $\begin{array}{l}Q_{20} \\
Q^{8} \\
Q_{59}\end{array}$ & $\begin{array}{c}\text { NF } \\
34.46 \\
25.76 \\
38.50 \\
\text { NF } \\
33.33\end{array}$ \\
\hline $\begin{array}{l}\text { Workers } \\
4 \text { or Fewer } \\
5 \text { to } 9 \\
10 \text { to } 19 \\
20 \text { to } 49 \\
50 \text { to } 99 \\
100 \text { to } 249 \\
250 \text { or More }\end{array}$ & $\begin{array}{l}Q^{Q} \\
Q_{16} \\
Q^{16} \\
Q_{99} \\
225\end{array}$ & 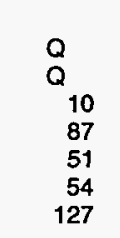 & $\begin{array}{l}\mathbf{Q} \\
\mathbf{Q} \\
\mathbf{Q} \\
\mathbf{Q} \\
\mathbf{Q} \\
\mathbf{Q} \\
\mathbf{Q}\end{array}$ & $\begin{array}{l}Q \\
Q \\
Q \\
Q \\
Q \\
Q \\
Q_{38}\end{array}$ & $\begin{array}{c}\mathrm{NF} \\
\mathrm{NF} \\
32.36 \\
\mathrm{NF} \\
\mathrm{NF} \\
34.67 \\
34.30\end{array}$ \\
\hline 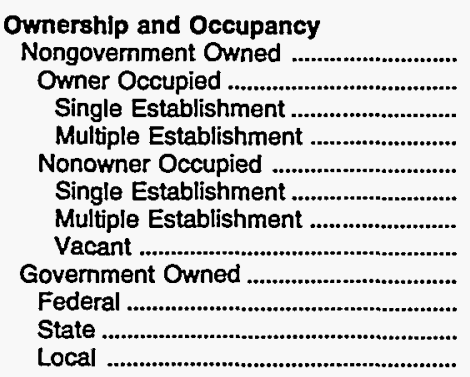 & $\begin{array}{l}284 \\
263 \\
228 \\
35 \\
Q \\
Q \\
Q \\
Q \\
301 \\
Q \\
201 \\
35\end{array}$ & $\begin{array}{l}141 \\
130 \\
108 \\
21 \\
Q \\
Q \\
Q^{21} \\
214 \\
Q \\
146 \\
19\end{array}$ & $\begin{array}{l}Q \\
Q \\
Q \\
Q \\
Q \\
Q \\
Q \\
N C \\
\mathbf{5 1} \\
Q \\
Q \\
Q\end{array}$ & $\begin{array}{l}65 \\
58 \\
53 \\
Q^{4} \\
Q \\
Q \\
Q \\
Q^{36} \\
Q^{21}\end{array}$ & $\begin{array}{l}27.16 \\
28.65 \\
33.34 \\
23.86 \\
\mathrm{NF} \\
\mathrm{NF} \\
\mathrm{NF} \\
\mathrm{NF} \\
28.07 \\
\mathrm{NF} \\
35.62 \\
42.67\end{array}$ \\
\hline
\end{tabular}

See footnotes at end of table. 
Table B12. Consumption of District Heat by End Use, 1989 (Continued)

\begin{tabular}{|c|c|c|c|c|c|}
\hline \multirow{3}{*}{$\begin{array}{c}\begin{array}{c}\text { Bullding } \\
\text { Characterlstics }\end{array} \\
\text { RSE Column Factor: }\end{array}$} & \multicolumn{4}{|c|}{$\begin{array}{l}\text { District Heat Consumption } \\
\text { (trillion Btu) }\end{array}$} & \multirow{3}{*}{$\begin{array}{l}\text { RSE } \\
\text { Row } \\
\text { Factor }\end{array}$} \\
\hline & Total & $\begin{array}{l}\text { Space } \\
\text { Heating }\end{array}$ & $\begin{array}{c}\text { Water } \\
\text { Heating }\end{array}$ & Othera & \\
\hline & 1.0 & NF & NF & NF & \\
\hline \multicolumn{6}{|l|}{ Multlbullding Facility } \\
\hline 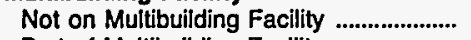 & 58 & 38 & $\mathbf{Q}$ & 7 & 30.76 \\
\hline $\begin{array}{l}\text { Part of Multibuilding Facility ....................... } \\
\text { On Facility with Central }\end{array}$ & 527 & 317 & 116 & 94 & 24.33 \\
\hline 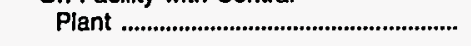 & 476 & 277 & 108 & 91 & 25.31 \\
\hline \multicolumn{6}{|l|}{$\begin{array}{l}\text { Percent Vacant at Least Three } \\
\text { Months }\end{array}$} \\
\hline 0 & 412 & 261 & 75 & 75 & 22.89 \\
\hline 1 to 50 & 131 & 67 & $\mathrm{Q}$ & 17 & 38.91 \\
\hline 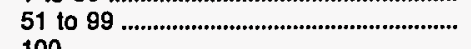 & $\mathbf{Q}$ & $Q$ & $\vec{Q}$ & $Q$ & $\mathrm{NF}$ \\
\hline 100 & & & $\mathbf{Q}$ & $\mathbf{Q}$ & NF \\
\hline \multicolumn{6}{|l|}{ Months in Use Out of Past 12 Months } \\
\hline $\begin{array}{l}0 \text { to } 8 \\
9 \text { to } 11 \\
12\end{array}$ & $\begin{array}{l}Q \\
Q \\
555\end{array}$ & $\begin{array}{l}Q \\
Q \\
335\end{array}$ & $\begin{array}{l}Q \\
Q \\
121\end{array}$ & $\begin{array}{l}Q_{99} \\
Q_{99}\end{array}$ & $\begin{array}{l}\mathrm{NF} \\
\mathrm{NF} \\
21.28\end{array}$ \\
\hline \multicolumn{6}{|l|}{$\begin{array}{l}\text { LOCATION } \\
\text { Census Reglon }\end{array}$} \\
\hline 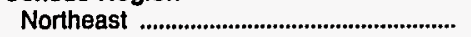 & 179 & 111 & 39 & 29 & 34.50 \\
\hline Midwest & 159 & 70 & $\mathbf{Q}$ & 30 & 39.48 \\
\hline South & 126 & 90 & & 21 & 44.55 \\
\hline 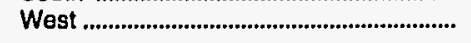 & 121 & 84 & 17 & $Q$ & 39.73 \\
\hline \multicolumn{6}{|l|}{$\begin{array}{l}\text { Census Division } \\
\text { Northeast }\end{array}$} \\
\hline 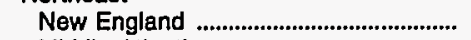 & $\mathbf{Q}$ & $\mathbf{Q}$ & $\mathbf{Q}$ & $\mathrm{Q}$ & NF \\
\hline 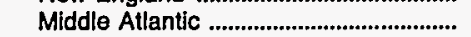 & 127 & 87 & 22 & 18 & 27.22 \\
\hline \multicolumn{6}{|l|}{ Midwest } \\
\hline 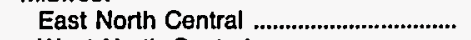 & 88 & 42 & $\mathbf{Q}$ & Q & 46.67 \\
\hline West North Central ......................................... & $\mathbf{Q}$ & Q & $\mathbf{Q}$ & 12 & NF \\
\hline \multicolumn{6}{|l|}{ South } \\
\hline 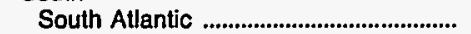 & $\mathbf{Q}$ & $\mathbf{Q}$ & $\mathbf{Q}$ & $\mathbf{Q}$ & NF \\
\hline 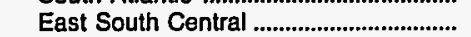 & $\vec{Q}$ & $\vec{Q}$ & $\vec{Q}$ & $\vec{Q}$ & NF \\
\hline 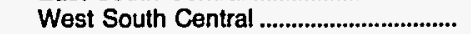 & $\mathbf{Q}$ & $\mathbf{Q}$ & $\mathbf{Q}$ & $\mathbf{Q}$ & NF \\
\hline \multicolumn{6}{|l|}{ West } \\
\hline 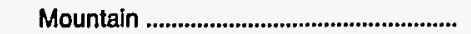 & $\mathbf{Q}$ & 55 & 7 & Q & NF \\
\hline Pacific .......................................................... & $\overrightarrow{\mathbf{Q}}$ & $\mathrm{Q}$ & 9 & $\mathbf{Q}$ & NF \\
\hline \multirow{2}{*}{\multicolumn{6}{|c|}{$\begin{array}{l}\text { Cllmate Zone: 45-Year Average } \\
\text { Under 2,000 CDD and - }\end{array}$}} \\
\hline & & & & & \\
\hline Over 7,000 HDD & $\mathbf{Q}$ & $\mathbf{Q}$ & $\mathbf{Q}$ & $Q$ & NF \\
\hline 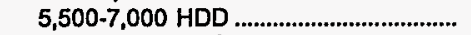 & 199 & 120 & Q & 43 & 34.35 \\
\hline 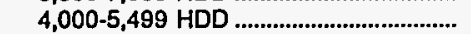 & 152 & 84 & $\mathbf{Q}$ & 24 & 40.29 \\
\hline 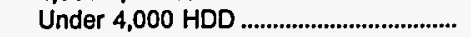 & 83 & $Q$ & $\mathrm{Q}$ & $Q$ & 46.05 \\
\hline 2,000 CDD or More and -- & & & & & \\
\hline Under 4,000 HDD & $\mathbf{Q}$ & Q & $\mathbf{Q}$ & Q & NF \\
\hline \multicolumn{6}{|l|}{1989 Degree-Days } \\
\hline Over 7,000 HDD ............................................ & $\mathbf{Q}$ & 74 & $\mathbf{Q}$ & Q & NF \\
\hline $5,500-7,000$ HDD & 254 & 136 & $\bar{Q}$ & 53 & 32.06 \\
\hline $4,000-5,499$ HDD ............................................ & 84 & 55 & 16 & $\mathrm{Q}$ & 29.91 \\
\hline Under 4,000 HDD & $\mathbf{Q}$ & $\mathbf{Q}$ & 12 & Q & NF \\
\hline 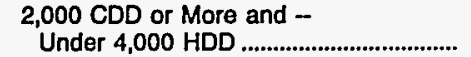 & $\mathbf{Q}$ & 0 & $Q$ & $\mathbf{Q}$ & $\mathrm{NF}$ \\
\hline \multicolumn{6}{|l|}{ STRUCTURE } \\
\hline Floors & & & & & \\
\hline 1 & & Q & Q & Q & NF \\
\hline 2 & 70 & 48 & $Q$ & $Q^{2}$ & 44.61 \\
\hline 3 to 6 & $Q$ & $\begin{array}{r}68 \\
109\end{array}$ & $\begin{array}{l}Q \\
Q\end{array}$ & $Q^{26}$ & $\begin{array}{c}N F \\
40.67\end{array}$ \\
\hline 4 to 6 More & $\begin{array}{l}168 \\
177\end{array}$ & $\begin{array}{r}109 \\
96\end{array}$ & 0 & 27 & 37.46 \\
\hline
\end{tabular}

See footnotes at end of table. 
Table B12. Consumption of District Heat by End Use, 1989 (Continued)

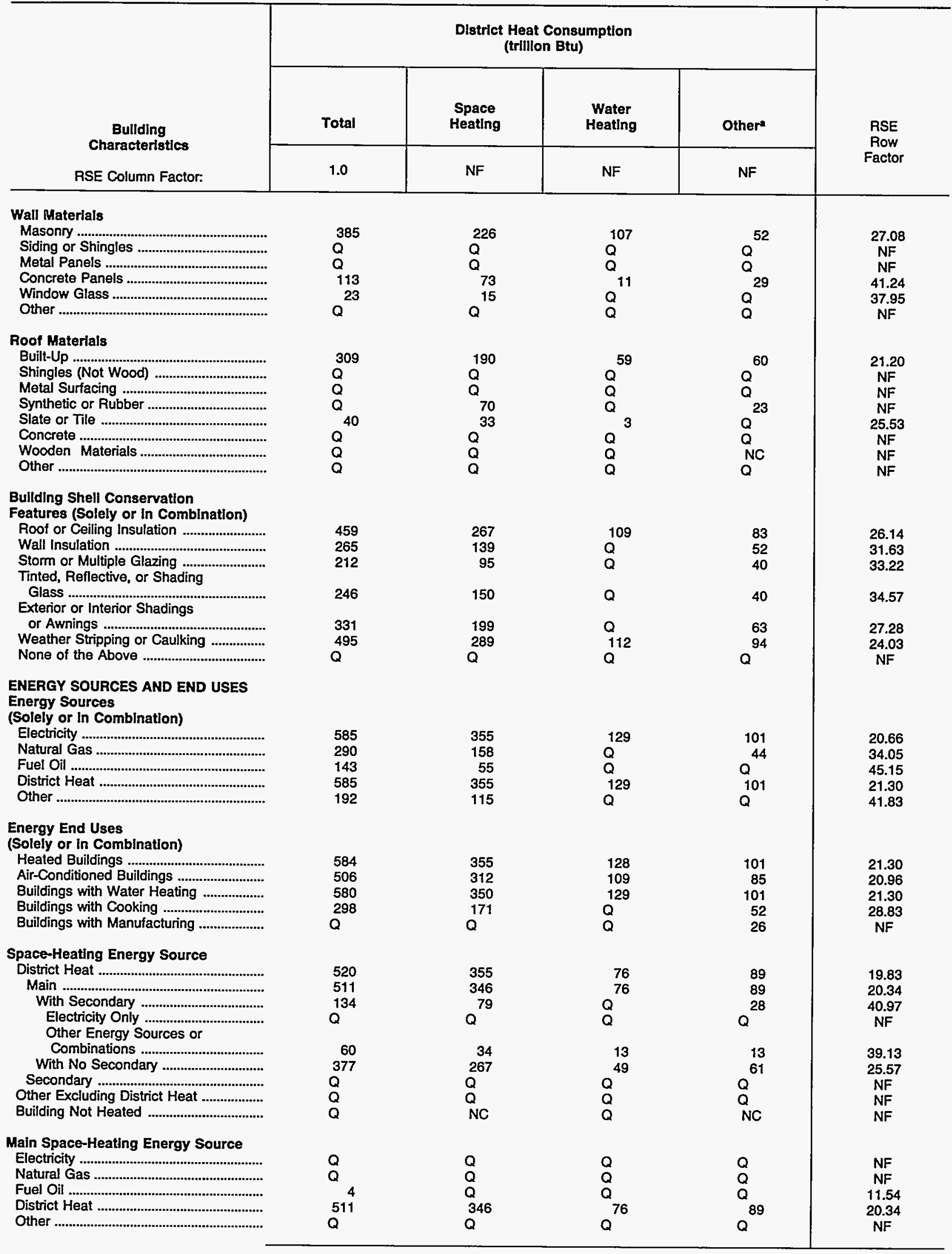

See footnotes at end of table. 
Table B12. Consumption of District Heat by End Use, 1989 (Continued)

\begin{tabular}{|c|c|c|c|c|c|}
\hline \multirow{3}{*}{$\begin{array}{c}\text { Bullding } \\
\text { Characterlstics } \\
\text { RSE Column Factor: }\end{array}$} & \multicolumn{4}{|c|}{$\begin{array}{l}\text { District Heat Consumption } \\
\text { (trillion Btu) }\end{array}$} & \multirow{3}{*}{$\begin{array}{l}\text { RSE } \\
\text { Row } \\
\text { Factor }\end{array}$} \\
\hline & Total & $\begin{array}{l}\text { Space } \\
\text { Heating }\end{array}$ & $\begin{array}{l}\text { Water } \\
\text { Heating }\end{array}$ & Others & \\
\hline & 1.0 & NF & NF & NF & \\
\hline 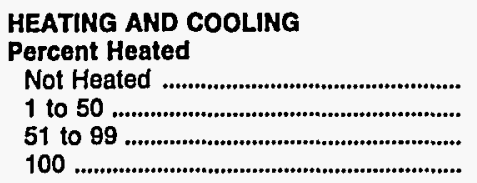 & $\begin{array}{l}Q^{Q} \\
Q_{59} \\
519\end{array}$ & $\begin{array}{l}Q \\
Q \\
48 \\
304\end{array}$ & $\begin{array}{l}Q \\
Q \\
8 \\
119\end{array}$ & $\begin{array}{l}\text { NC } \\
\mathbf{Q} \\
\mathbf{Q} \\
96\end{array}$ & $\begin{array}{l}\mathrm{NF} \\
\mathrm{NF} \\
32.59 \\
22.47\end{array}$ \\
\hline 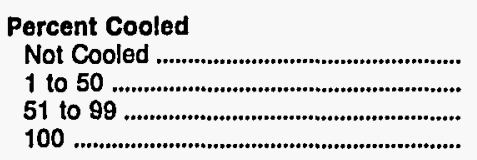 & $\begin{array}{l}Q \\
113 \\
137 \\
256\end{array}$ & $\begin{array}{l}Q \\
75 \\
100 \\
137\end{array}$ & $Q_{16}$ & $\begin{array}{l}Q \\
Q \\
Q \\
47\end{array}$ & $\begin{array}{c}\text { NF } \\
33.77 \\
25.15 \\
28.59\end{array}$ \\
\hline 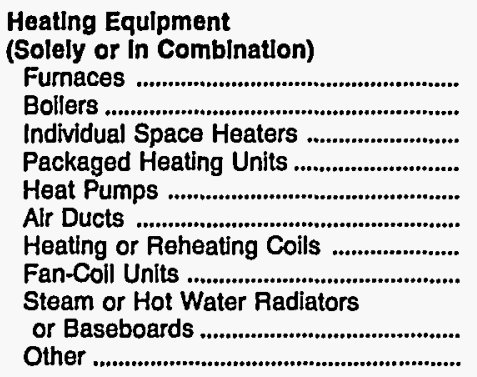 & $\begin{array}{l}Q_{50} \\
189 \\
Q^{18} \\
Q \\
430 \\
339 \\
267 \\
\\
378 \\
Q\end{array}$ & $\begin{array}{l}Q \\
Q \\
114 \\
Q \\
Q \\
265 \\
208 \\
156 \\
\\
218 \\
Q\end{array}$ & $\begin{array}{l}Q \\
Q \\
Q \\
Q \\
Q \\
101 \\
Q \\
Q \\
Q^{85}\end{array}$ & $\begin{array}{l}\mathrm{NC}^{\mathrm{Q}} \\
{ }^{30} \\
\mathrm{Q}^{\mathrm{Q}} \\
65 \\
53 \\
48 \\
\mathrm{Q}^{75}\end{array}$ & $\begin{array}{c}N F \\
49.84 \\
40.89 \\
N F \\
N F \\
25.56 \\
27.57 \\
36.14 \\
\\
26.62 \\
N F\end{array}$ \\
\hline 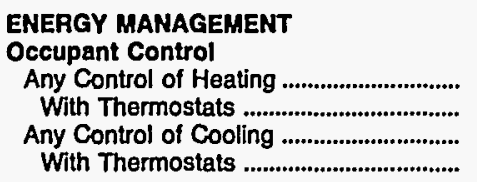 & $\begin{array}{l}206 \\
203 \\
223 \\
216\end{array}$ & $\begin{array}{r}92 \\
89 \\
113 \\
108\end{array}$ & $\begin{array}{l}\mathbf{Q} \\
\mathbf{Q} \\
\mathbf{Q} \\
\mathbf{Q}\end{array}$ & $\begin{array}{l}40 \\
40 \\
42 \\
42\end{array}$ & $\begin{array}{l}37.85 \\
38.28 \\
33.97 \\
34.76\end{array}$ \\
\hline 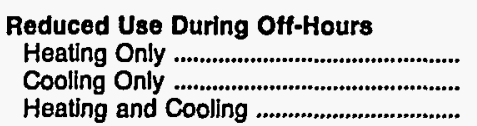 & $\begin{array}{l}Q \\
Q \\
289\end{array}$ & $\begin{array}{r}39 \\
19 \\
203\end{array}$ & $\begin{array}{l}\mathbf{Q} \\
\mathbf{Q} \\
\mathbf{Q}\end{array}$ & $\begin{array}{l}Q_{27} \\
33\end{array}$ & $\begin{array}{c}\mathrm{NF} \\
\mathrm{NF} \\
33.27\end{array}$ \\
\hline 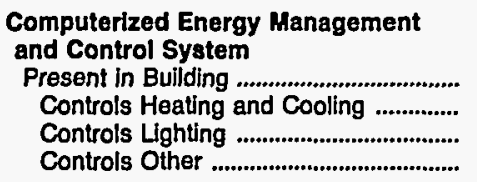 & $\begin{array}{l}272 \\
271 \\
Q \\
Q\end{array}$ & $\begin{array}{l}166 \\
165 \\
Q_{12}\end{array}$ & ${ }^{Q}{ }^{Q}$ & $Q^{35}$ & $\begin{array}{l}32.90 \\
32.98 \\
\mathrm{NF} \\
\mathrm{NF}\end{array}$ \\
\hline 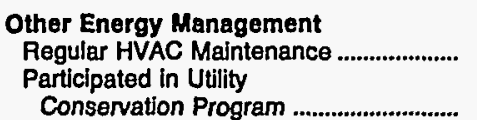 & $\begin{array}{l}530 \\
154\end{array}$ & $\begin{array}{r}319 \\
96\end{array}$ & $\begin{array}{l}117 \\
Q\end{array}$ & $\begin{array}{l}95 \\
Q^{95}\end{array}$ & $\begin{array}{l}21.97 \\
38.56\end{array}$ \\
\hline
\end{tabular}

- Includes cooking and cooling.

$N C=$ No cases in responding sample.

$\mathrm{NF}=$ No applicable RSE row/column factor.

$\mathrm{Q}=$ Data withheld because the Relative Standard Error (RSE) was greater than 50 percent, or data were reported for fewer than 20 buildings.

Notes: - To obtain the RSE percentage for any table cell, multiply the corresponding RSE column and RSE row factors. - See Glossary for explanation of abbreviations and definitions of terms used in this report. - Because of rounding, data may not sum to totals.

Source: Energy Information Administration, Office of Energy Markets and End Use, Forms EIA-871A through F of the 1989 Commercial Buildings Energy Consumption Survey. 


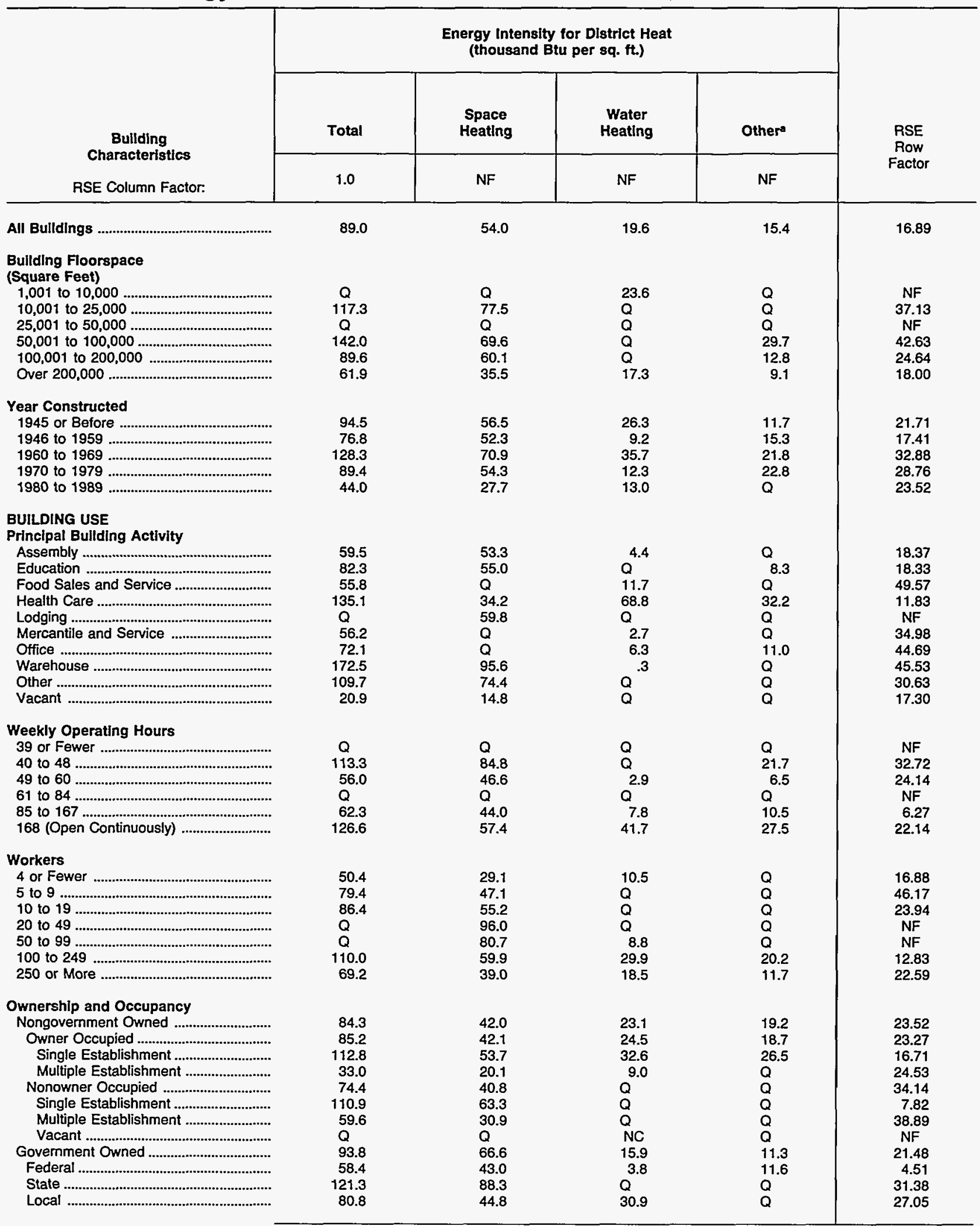

See footnote at end of table. 
Table B13. Energy End-Use Intensities for District Heat, 1989 (Continued)

\begin{tabular}{|c|c|c|c|c|c|}
\hline \multirow{3}{*}{$\begin{array}{c}\text { Bullding } \\
\text { Characteristles } \\
\text { RSE Column Factor: }\end{array}$} & \multicolumn{4}{|c|}{$\begin{array}{l}\text { Energy Intensity for District Heat } \\
\text { (thousand Btu per sq. ft.) }\end{array}$} & \multirow{3}{*}{$\begin{array}{l}\text { RSE } \\
\text { Row } \\
\text { Factor }\end{array}$} \\
\hline & Total & $\begin{array}{l}\text { Space } \\
\text { Heating }\end{array}$ & $\begin{array}{l}\text { Water } \\
\text { Heating }\end{array}$ & Other & \\
\hline & 1.0 & NF & NF & NF & \\
\hline \multicolumn{6}{|l|}{ Multibullding Facillty } \\
\hline Not on Multibullding Facility .......................... & 44.9 & 29.6 & 9.8 & 5.5 & 34.61 \\
\hline $\begin{array}{l}\text { Part of Multibuilding Facility ....................... } \\
\text { On Facility with Central }\end{array}$ & 99.7 & 59.9 & 22.0 & 17.8 & 16.93 \\
\hline 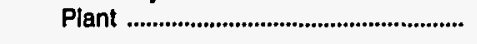 & 107.3 & 62.4 & 24.2 & 20.6 & 17.80 \\
\hline \multicolumn{6}{|l|}{$\begin{array}{l}\text { Percent Vacant at Least Three } \\
\text { Months }\end{array}$} \\
\hline 0 & 102.8 & 65.3 & 18.7 & 18.8 & 19.78 \\
\hline 1 to 50 & 71.4 & 36.7 & 25.5 & 9.2 & 25.75 \\
\hline 51 to 99 & 66.2 & 42.3 & $\mathbf{Q}$ & 16.0 & 7.15 \\
\hline 100 & 36.4 & 19.8 & $Q$ & 2.1 & 17.77 \\
\hline \multicolumn{6}{|l|}{ Months in Use Out of Past 12 Months } \\
\hline 0 to 8 & 35.7 & 15.1 & 13.0 & $\mathbf{Q}$ & 17.02 \\
\hline 9 to 11 & 66.6 & Q & Q & $\overline{\mathbf{Q}}$ & 37.09 \\
\hline 12 & 92.2 & 55.6 & 20.1 & 16.5 & 16.69 \\
\hline \multicolumn{6}{|l|}{$\begin{array}{l}\text { LOCATION } \\
\text { Census Reglon }\end{array}$} \\
\hline 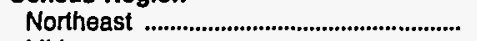 & 80.1 & 49.5 & 17.5 & $\mathbf{Q}$ & 36.85 \\
\hline 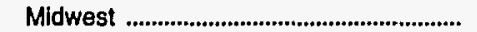 & 105.3 & 46.6 & 38.7 & 20.0 & 12.36 \\
\hline South & Q & $\mathbf{Q}$ & $\mathbf{Q}$ & 13.4 & $\mathrm{NF}$ \\
\hline West & 97.1 & 67.2 & Q & 16.4 & 13.69 \\
\hline \multirow{2}{*}{\multicolumn{6}{|c|}{$\begin{array}{l}\text { Census Divlsion } \\
\text { Northeast }\end{array}$}} \\
\hline & & & & & \\
\hline 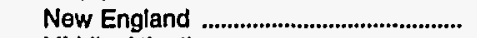 & $\mathbf{Q}$ & Q & Q & $Q$ & NF \\
\hline 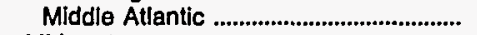 & 68.3 & 46.6 & 12.0 & $\mathbf{Q}$ & 27.89 \\
\hline \multicolumn{6}{|l|}{ Midwest } \\
\hline East North Central ............................................ & 104.0 & 49.6 & 33.4 & 21.0 & 16.02 \\
\hline 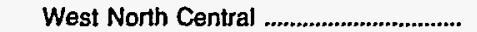 & 106.9 & 42.7 & 45.4 & 18.8 & 14.36 \\
\hline \multicolumn{6}{|l|}{ South } \\
\hline 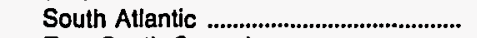 & $\mathbf{Q}$ & $\mathbf{Q}$ & $Q$ & $Q$ & NF \\
\hline 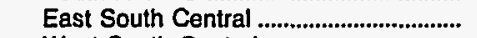 & $\bar{Q}$ & $\bar{Q}$ & $\bar{Q}$ & $\bar{Q}$ & NF \\
\hline 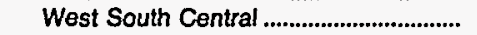 & $Q$ & $\mathbf{Q}$ & $\mathbf{Q}$ & $\vec{Q}$ & NF \\
\hline \multicolumn{6}{|l|}{ West } \\
\hline 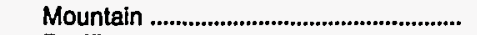 & 99.1 & 74.8 & $\mathbf{Q}$ & 14.1 & 12.73 \\
\hline 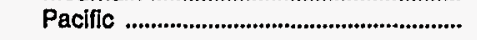 & 94.4 & 56.4 & 18.3 & $Q$ & 22.50 \\
\hline \multirow{2}{*}{\multicolumn{6}{|c|}{$\begin{array}{l}\text { Cllmate Zone: } 45-\text { Year Average } \\
\text { Under 2,000 CDD and -- }\end{array}$}} \\
\hline & & & & & \\
\hline Over 7,000 HDD .......................................... & 206.3 & 117.8 & Q & Q & 43.76 \\
\hline $\begin{array}{l}5,500-7,000 \text { HDD } \\
4,000-5,499 \text { HDD }\end{array}$ & 90.4 & 54.7 & $Q$ & 19.3 & 15.92 \\
\hline 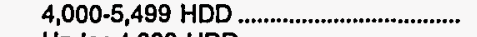 & 71.8 & 39.8 & 20.6 & $Q$ & 28.13 \\
\hline 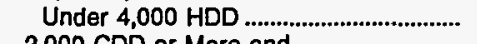 & 70.6 & Q & 13.2 & $\bar{Q}$ & 40.97 \\
\hline $\begin{array}{l}\text { 2,000 CDD or More and }- \\
\text { Under 4,000 HDD }\end{array}$ & & & & & \\
\hline 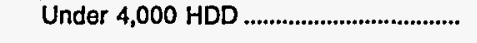 & $\mathbf{Q}$ & Q & $\mathbf{Q}$ & Q & NF \\
\hline \multirow{2}{*}{\multicolumn{6}{|c|}{$\begin{array}{l}1989 \text { Degree-Days } \\
\text { Under 2,000 CDD and - }\end{array}$}} \\
\hline & & & & & \\
\hline 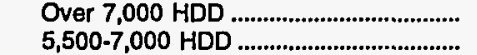 & $\begin{array}{l}Q \\
92.5\end{array}$ & $\begin{array}{l}91.1 \\
49.4\end{array}$ & $Q$ & $Q$ & $\begin{array}{c}\mathrm{NF} \\
1570\end{array}$ \\
\hline 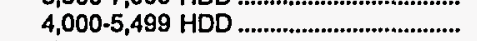 & 63.0 & $Q$ & $\begin{array}{l}20.1 \\
12.3\end{array}$ & $Q^{19.3}$ & $\begin{array}{l}15.19 \\
33.84\end{array}$ \\
\hline 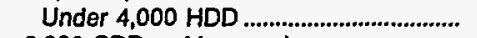 & 67.2 & $\mathbf{Q}$ & 11.3 & $\vec{Q}$ & 46.66 \\
\hline 2,000 CDD or More and -- & & & & & \\
\hline Under 4,000 HDD & $\mathbf{Q}$ & $\mathbf{Q}$ & $\mathbf{Q}$ & Q & NF \\
\hline \multicolumn{6}{|l|}{$\begin{array}{l}\text { STRUCTURE } \\
\text { Floors }\end{array}$} \\
\hline Ploors & & 111.7 & $\mathbf{Q}$ & $\mathbf{Q}$ & NF \\
\hline 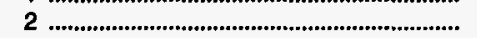 & 82.8 & 56.2 & $\vec{Q}$ & $\mathbf{Q}$ & 27.67 \\
\hline 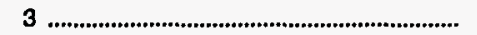 & $\mathbf{Q}$ & 59.3 & $\bar{Q}$ & 22.6 & NF \\
\hline 4 to 6 & 101.2 & 65.8 & $\mathbf{Q}$ & 13.4 & 15.37 \\
\hline 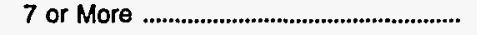 & 67.9 & 36.6 & 21.1 & 10.2 & 24.83 \\
\hline
\end{tabular}

See footnote at end of table. 


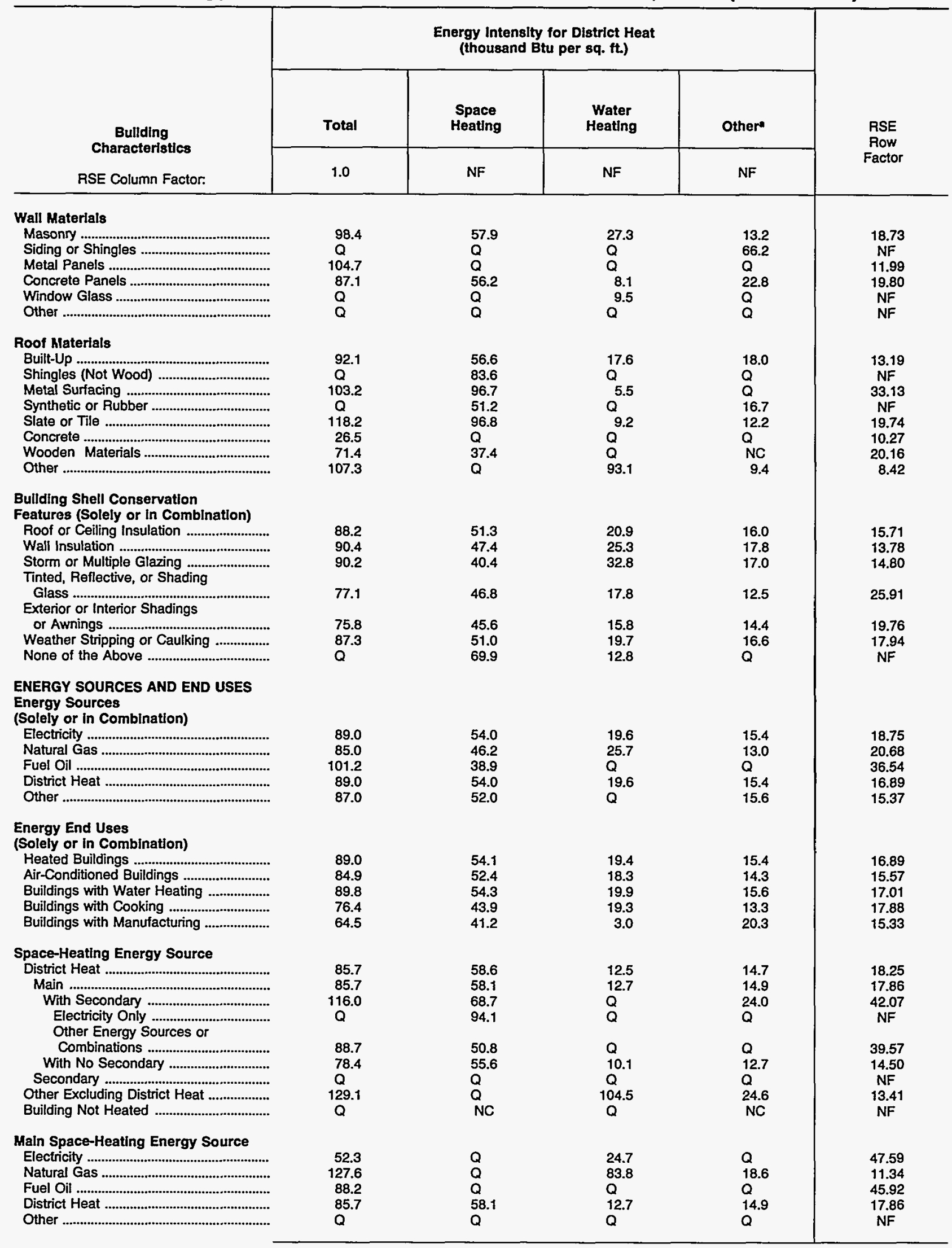

See footnote at end of table. 
Table B13. Energy End-Use Intensities for District Heat, 1989 (Continued)

\begin{tabular}{|c|c|c|c|c|c|}
\hline \multirow{3}{*}{$\begin{array}{l}\text { Bullding } \\
\text { Characteristics } \\
\text { RSE Column Factor: }\end{array}$} & \multicolumn{4}{|c|}{$\begin{array}{l}\text { Energy Intensity for District Heat } \\
\text { (thousand Btu per sq. ft.) }\end{array}$} & \multirow{3}{*}{$\begin{array}{l}\text { RSE } \\
\text { Row } \\
\text { Factor }\end{array}$} \\
\hline & \multirow{2}{*}{ Total } & \multirow{2}{*}{$\begin{array}{c}\text { Space } \\
\text { Heating }\end{array}$} & \multirow{2}{*}{$\begin{array}{c}\begin{array}{c}\text { Water } \\
\text { Heating }\end{array} \\
\mathrm{NF}\end{array}$} & \multirow{2}{*}{$\begin{array}{c}\text { Other" } \\
\mathrm{NF}\end{array}$} & \\
\hline & & & & & \\
\hline 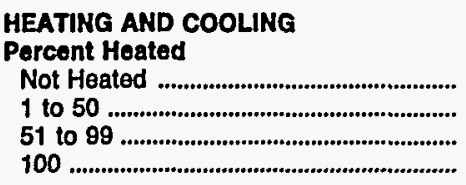 & $\begin{array}{l}Q \\
46.9 \\
50.2 \\
98.8\end{array}$ & $\begin{array}{l}Q \\
Q \\
40.8 \\
57.9\end{array}$ & $\begin{array}{r}Q \\
6.4 \\
6.9 \\
22.6\end{array}$ & $\begin{array}{l}\text { NC } \\
Q \\
Q \\
18.3\end{array}$ & $\begin{array}{l}\text { NF } \\
41.52 \\
28.01 \\
18.75\end{array}$ \\
\hline 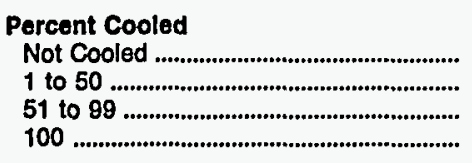 & $\begin{array}{r}Q \\
104.1 \\
77.6 \\
82.4\end{array}$ & $\begin{array}{l}69.7 \\
68.6 \\
56.7 \\
44.2\end{array}$ & $\begin{array}{l}Q \\
15.1 \\
12.2 \\
22.9\end{array}$ & $\begin{array}{l}Q \\
Q \\
8.7 \\
15.3\end{array}$ & $\begin{array}{c}\text { NF } \\
35.07 \\
15.35 \\
28.04\end{array}$ \\
\hline \multicolumn{6}{|l|}{$\begin{array}{l}\text { Heating Equipment } \\
\text { (Solely or In Combination) }\end{array}$} \\
\hline 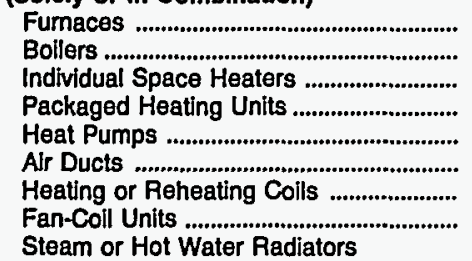 & $\begin{array}{l}Q \\
Q \\
84.9 \\
82.9 \\
73.9 \\
76.8 \\
72.8 \\
85.9\end{array}$ & $\begin{array}{l}Q \\
Q \\
51.3 \\
51.3 \\
62.0 \\
47.2 \\
44.7 \\
50.1\end{array}$ & $\begin{array}{l}Q \\
Q \\
Q \\
Q \\
9.2 \\
18.0 \\
16.6 \\
20.3\end{array}$ & $\begin{array}{l}N C \\
Q \\
13.6 \\
Q \\
Q \\
11.6 \\
11.4 \\
15.4\end{array}$ & $\begin{array}{l}\mathrm{NF} \\
\mathrm{NF} \\
16.32 \\
37.04 \\
27.99 \\
17.11 \\
18.14 \\
13.52\end{array}$ \\
\hline or Baseboards & $\begin{array}{r}96.9 \\
128.7\end{array}$ & $\begin{array}{l}55.9 \\
\mathbf{Q}\end{array}$ & $\begin{array}{l}21.7 \\
73.9\end{array}$ & $\stackrel{19.2}{Q}$ & $\begin{array}{l}19.24 \\
22.08\end{array}$ \\
\hline \multicolumn{6}{|l|}{$\begin{array}{l}\text { ENERGY MANAGEMENT } \\
\text { Occupant Control }\end{array}$} \\
\hline 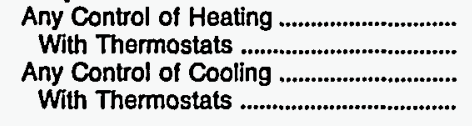 & $\begin{array}{r}106.9 \\
107.8 \\
99.5 \\
100.1\end{array}$ & $\begin{array}{l}47.6 \\
47.2 \\
50.4 \\
49.9\end{array}$ & $\begin{array}{l}38.6 \\
39.4 \\
30.3 \\
30.6\end{array}$ & $\begin{array}{l}20.8 \\
21.2 \\
18.8 \\
19.5\end{array}$ & $\begin{array}{l}25.14 \\
25.26 \\
17.32 \\
17.60\end{array}$ \\
\hline 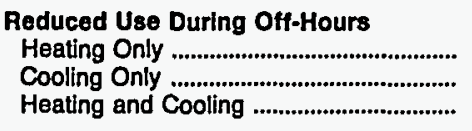 & $\begin{array}{l}Q \\
130.1 \\
69.6\end{array}$ & $\begin{array}{l}71.7 \\
46.9 \\
48.7\end{array}$ & $\begin{array}{l}Q \\
Q \\
Q\end{array}$ & $\begin{array}{l}Q \\
66.4 \\
7.9\end{array}$ & $\begin{array}{c}\mathrm{NF} \\
47.68 \\
20.28\end{array}$ \\
\hline \multicolumn{6}{|l|}{$\begin{array}{l}\text { Computerized Energy Management } \\
\text { and Control System }\end{array}$} \\
\hline 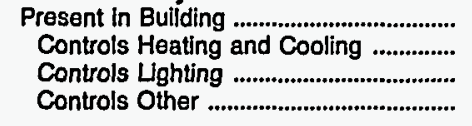 & $\begin{array}{l}72.5 \\
72.7 \\
72.4 \\
88.9\end{array}$ & $\begin{array}{l}44.1 \\
44.4 \\
45.7 \\
20.7\end{array}$ & $\begin{array}{r}19.1 \\
19.2 \\
5.8 \\
57.7\end{array}$ & $\begin{array}{r}9.3 \\
9.1 \\
20.9 \\
10.5\end{array}$ & $\begin{array}{l}21.37 \\
21.50 \\
22.10 \\
14.93\end{array}$ \\
\hline $\begin{array}{l}\text { Other Energy Management } \\
\text { Regular HVAC Maintenance ..................... } \\
\text { Participated in Utility }\end{array}$ & 87.7 & 52.7 & 19.3 & 15.7 & 17.26 \\
\hline 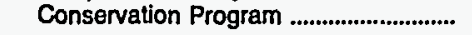 & 92.3 & 57.5 & $\mathbf{Q}$ & 13.6 & 35.95 \\
\hline
\end{tabular}

- Includes cooking and cooling.

$N C=$ No cases in responding sample.

$\mathrm{NF}=$ No applicable RSE row/column factor.

$Q=$ Data withheld because the Relative Standard Error (RSE) was greater than 50 percent, or data were reported for fewer than 20 buildings.

Notes: - To obtain the RSE percentage for any table cell, multiply the corresponding RSE column and RSE row factors. - See Glossary for explanation of abbreviations and definitions of terms used in this report. - Because of rounding, data may not sum to totals.

Source: Energy Information Administration, Office of Energy Markets and End Use, Forms ElA-871A through F of the 1989 Commercial Buildings Energy Consumption Survey. 
, 
Appendix C

\section{U.S. Climate Zones and Census Regions and Divisions Maps}

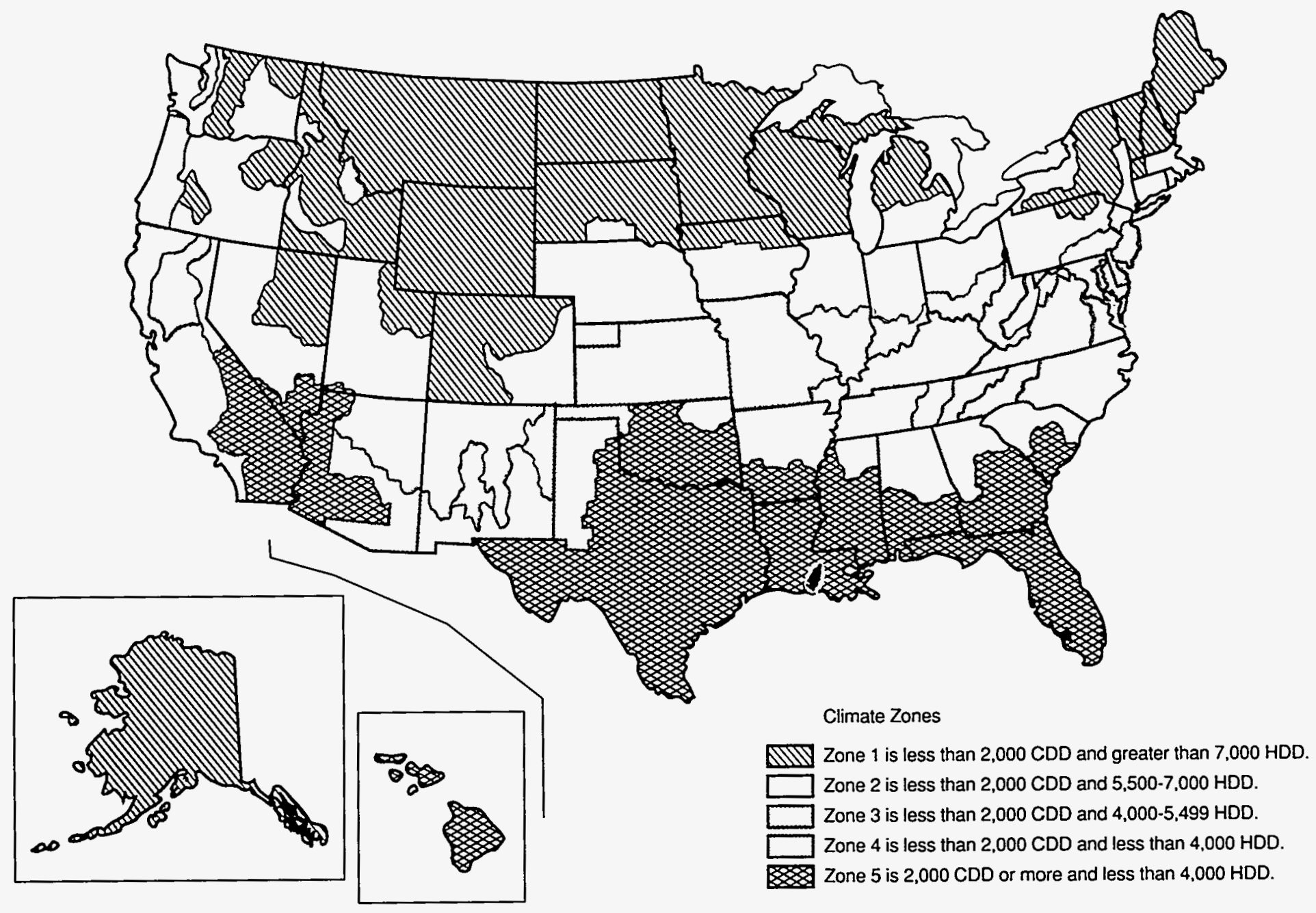




\section{U.S. Census Regions and Divisions}

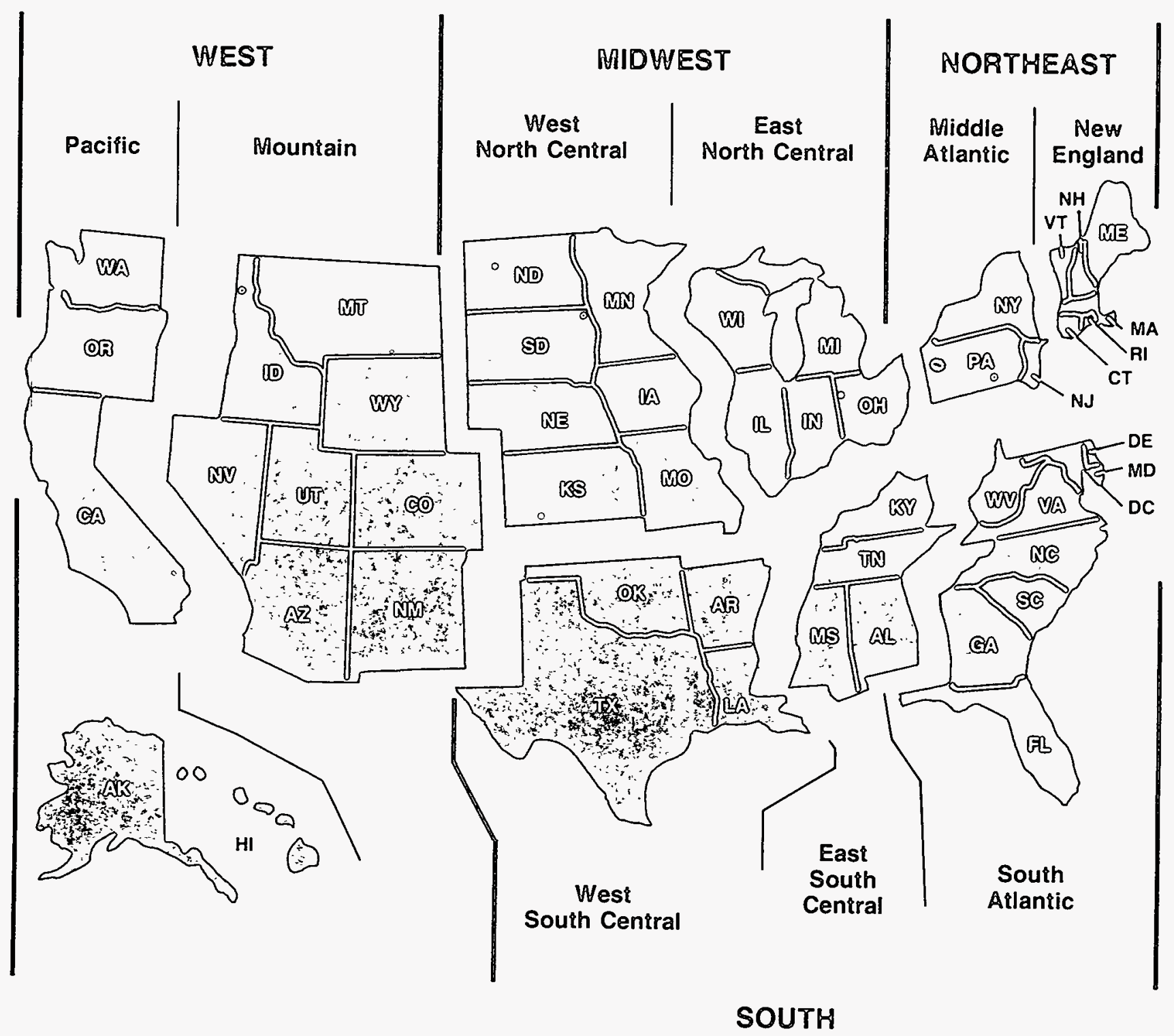




\section{Glossary}

Active Solar: As an energy source, energy from the sun collected and stored using mechanical pumps or fans to circulate heat-laden fluids or air between solar collectors and the building. Examples include the use of solar collectors for water or space heating. The 1989 CBECS did not gather consumption and expenditures data for active solar. Data on the passive collection of solar energy, such as by trombe walls, were not collected on the 1989 CBECS. (See Energy Source.)

\section{Air Conditioning: See Cooling.}

Air Ducts or Air-Handling Units: A vehicle for channeling warm or cool air to different parts of a building. The process of moving the conditioned air often involves passing air over heating or cooling coils and forcing it from a central location through ducts or air-handling units. Air-handling units are hidden in the walls or ceilings, where they use steam or hot water to heat the air or chilled water to cool the air, inside the duct work. (See Cooling, Duct, and Space Heating.)

Authorization Form: A form signed by the respondent from a building, authorizing energy supplier companies that serve the building to release information on the amounts and costs of energy consumed in the building during a specified period. (See Energy Supplier.)

\section{Ballast: See High-Efficiency Ballast.}

Barrel: A volumetric unit of measure for crude oil and petroleum products equivalent to 42 U.S. gallons. (See Gallon.)

Baseboard: As a type of heating equipment, a system in which either electric resistance coils or finned tubes carrying steam or hot water are mounted behind shallow panels along baseboards. Baseboards rely on passive convection to distribute heated air in the space. Electric baseboards are an example of an "Individual Space Heater." (See Individual Space Heater.)

Boiler: A type of space-heating equipment consisting of a vessel or tank where heat produced from the combustion of fuels such as natural gas, fuel oil, or coal is used to generate hot water or steam. Many buildings have their own boilers, while other buildings have steam or hot water piped in from a central plant. For this survey, only boilers inside the building (or serving only that particular building) are counted as part of the building's heating system. Steam or hot water piped into a building from a central plant is considered district heat. (See Furnace, HVAC, and District Heat.)

\section{Bottled Gas: See Liquefied Petroleum Gas (LPG) and Propane.}

British Thermal Unit: A unit of energy consumed by or delivered to a building. A Btu is defined as the amount of energy required to increase the temperature of 1 pound of water by 1 degree Fahrenheit, at normal atmospheric pressure. Energy consumption is expressed in Btu in this report to allow for consumption comparisons among fuels that are measured in different units. (See Metric Conversion Factors.)

\section{Btu: See British Thermal Unit.}


Btu Conversion Factors: The Btu conversion factors for this survey are as follows:

\begin{tabular}{lrl}
\hline & Btu Equivalent & \multicolumn{1}{c}{ Unit } \\
\hline Electricity & 3,412 & kilowatthour \\
Natural Gas & 1,030 & cubic foot \\
Distillate Fuel Oils (Nos. 1,2, and 4) & 138,690 & gallon \\
Residual Fuel Oils (Nos. 5 and 6) & 149,690 & gallon \\
Kerosene & 135,000 & gallon \\
District Heat (Steam and Hot Water) & 1,000 & pound \\
\hline
\end{tabular}

Note: Btu of district hot water have been converted into equivalent pounds of steam by using the conversion 1,000 Btu hot water $\approx 1$ pound steam.

Sources: Energy Information Administration, Monthly Energy Review (June 1991), pp. 125-129, for electricity, natural gas, distillate, residual, and kerosene; and Methodological Issues In the Nonresidential Buildings Energy Consumption Survey (September 1983), pp. 173-175, for district steam.

These are the Btu conversion factors for site energy consumption. For primary energy consumption, kilowatthours were converted to Btu at the rate of 10,236 Btu per kilowatthour, three times the site energy Btu. For district heat, pounds were converted to primary energy at the rate of 1,500 Btu per pound.

Building: For this survey, a structure totally enclosed by walls extending from the foundation to the roof, containing over 1,000 square feet of floorspace, and intended for human occupancy. Structures that were included in the survey as a specific exception were parking garages not totally enclosed by walls and a roof, as well as structures erected on pillars to elevate the first fully enclosed level, but leaving the sides at ground level open.

Excluded from the survey as nonbuildings were the following: structures (other than the exceptions just noted) that were not totally enclosed by walls and a roof (such as oil refineries, steel mills, and water towers); street lights, pumps, billboards, bridges, swimming pools, and construction sites; mobile homes and trailers, even if they housed commercial activity; and oil storage tanks. (See Commercial Building.)

\section{Building Floorspace: See Floorspace.}

Building Shell (Envelope): The thermal envelope of the building, that is, the roof, exterior walls, and bottom floors that enclose conditioned space through which thermal energy may be transferred to or from the exterior.

Building Shell Conservation Feature: A building feature designed to reduce the energy loss or gain through the shell or envelope of the building. The 1989 CBECS collected data on the following specific building shell energy conservation features: roof, ceiling or wall insulation; storm windows or double- or triple-paned glass (multiple glazing); tinted or reflective glass or shading films; exterior or interior shadings or awnings; and weather stripping or caulking. (See Roof or Ceiling Insulation, Wall Insulation, Reflective or Shading Glass or Film, Storm or Multiple Glazing, Building Shell (Envelope), Exterior or Interior Shadings or Awnings, and Weather Stripping or Caulking.)

Built-Up Roof: A roof covering consisting of several successive layers (each of which is called a ply) usually of roofing felt with moppings of hot asphalt between layers and topped by a mineral-surfaced layer or by gravel embedded in a heavy coat of asphalt.

Caulking: See Weather Stripping or Caulking.

CDD: See Cooling Degree-Days (CDD). 
Census Division: A geographic area consisting of several States defined by the U.S. Department of Commerce, Bureau of the Census. (See the Census Regions and Divisions map in Appendix C.) The States are grouped into nine divisions and four regions:

\begin{tabular}{|c|c|c|}
\hline Region & Division & States \\
\hline \multirow[t]{2}{*}{ Northeast } & New England & $\begin{array}{l}\text { Connecticut, Maine, Massachusetts, } \\
\text { New Hampshire, Vermont, and Rhode Island }\end{array}$ \\
\hline & Middle Atlantic & New Jersey, New York, and Pennsylvania \\
\hline \multirow[t]{2}{*}{ Midwest } & East North Central & $\begin{array}{l}\text { Illinois, Indiana, Michigan, } \\
\text { Ohio, and Wisconsin }\end{array}$ \\
\hline & West North Central & $\begin{array}{l}\text { Iowa, Kansas, Minnesota, Missouri, } \\
\text { Nebraska, North Dakota, and South Dakota }\end{array}$ \\
\hline \multirow[t]{3}{*}{ South } & South Atlantic & $\begin{array}{l}\text { Delaware, the District of Columbia, } \\
\text { Florida, Georgia, Maryland, North } \\
\text { Carolina, South Carolina, Virginia, } \\
\text { and West Virginia }\end{array}$ \\
\hline & East South Central & $\begin{array}{l}\text { Alabama, Kentucky, Mississippi, } \\
\text { and Tennessee }\end{array}$ \\
\hline & West South Central & $\begin{array}{l}\text { Arkansas, Louisiana, Oklahoma, } \\
\text { and Texas }\end{array}$ \\
\hline \multirow[t]{2}{*}{ West } & Mountain & $\begin{array}{l}\text { Arizona, Colorado, Idaho, Montana, } \\
\text { Nevada, New Mexico, Utah, and Wyoming }\end{array}$ \\
\hline & Pacific & $\begin{array}{l}\text { Alaska, California, Hawaii, Oregon, } \\
\text { and Washington }\end{array}$ \\
\hline
\end{tabular}

Census Region: See Census Division and the Census Regions and Divisions map in Appendix C of this report.

Central Chiller: Any centrally located air-conditioning system that produces chilled water in order to cool air. The chilled water or cold air is then distributed throughout the building by using pipes or air ducts, or both. These systems are also commonly known as "chillers," "centrifugal chillers," "reciprocating chillers," or "absorption chillers." Chillers are generally located in or just outside the building they serve. Buildings receiving district chilled water are served by chillers located at central physical plants. (See Cooling, District Chilled Water, Central Physical Plant, and HVAC.)

Central Physical Plant: A plant that is owned by, and on the grounds of, a multibuilding facility and that provides district heating, district cooling, or electricity to other buildings on the same facility. To qualify as a central plant for this survey, the plant must provide district heat, district chilled water, or electricity to at least one other building. The central physical plant may be by itself in a separate building or may be located in a building where other activities occur. (See Multibuilding Facility; District Heat; and District Chilled Water.)

Chiller: See Central Chiller.

Climate Zone: One of five climatically distinct areas defined by long-term weather conditions affecting the heating and cooling loads in buildings. The zones were developed by the Energy End Use and Integrated Statistics Division 
(EEUISD) from seven distinct climate categories originally identified by the American Institute of Architects (AIA) for the U.S. Department of Energy and the U.S. Department of Housing and Urban Development.

The zones were determined according to the 45-year average (1931-1975) of the annual heating and cooling degree-days (base 65 degrees Fahrenheit). An individual building was assigned to a climate zone according to the 45-year average annual degree-days for its NOAA Division. (See Heating Degree-Days (HDD), Cooling Degree-Days (CDD), Degree-Days 45-Year Average and NOAA Division.)

The zones are defined as follows:

\begin{tabular}{|c|c|c|}
\hline $\begin{array}{l}\text { Climate } \\
\text { Zone }\end{array}$ & $\begin{array}{c}\text { Average Annual } \\
\text { Cooling } \\
\text { Degree-Days }\end{array}$ & $\begin{array}{c}\text { Average Annual } \\
\text { Heating } \\
\text { Degree-Days }\end{array}$ \\
\hline 1 & Less than 2,000 & More than 7,000 \\
\hline 2 & Less than 2,000 & 5,500 to 7,000 \\
\hline 3 & Less than 2,000 & 4,000 to 5,499 \\
\hline 4 & Less than 2,000 & Less than 4,000 \\
\hline 5 & 2,000 or more & Less than 4,000 \\
\hline
\end{tabular}

Coal: In this report, the term includes anthracite, bituminous, and subbituminous coal, as well as the derivative of coal known as coke. The 1989 CBECS determined if coal was used in the commercial building but did not collect consumption and expenditure data on the use of coal as an energy source. (See Energy Source.)

Commercial: Neither residential, manufacturing, nor agricultural. (See Commercial Building.)

Commercial Building: A building with more than 50 percent of its floorspace used for commercial activities. Commercial buildings include, but are not limited to, stores, offices, schools, churches, gymnasiums, libraries, museums, hospitals, clinics, warehouses, and jails. Government buildings were included except for buildings on site with restricted access, such as some military bases or reservations. Farms and buildings located on farms (such as silos, grain elevators, and barns) were excluded from the survey. (See Building, Commercial, and Principal Building Activity.)

\section{Commercial Freezer: See Refrigeration Equipment.}

\section{Commercial Refrigeration Unit: See Refrigeration Equipment.}

Computer Area with Separate Air-Conditioning System: In this survey, this term is used to denote space specifically designed and equipped to meet the needs of computer equipment for controlled temperatures and/or humidity. The air-conditioning system for this area is separate from that used to control the environment in other parts of the building.

Computerized Energy Management and Control System: See Energy Management and Control System (EMCS).

Concrete Panel: A wall construction panel made of concrete, which is either prefabricated in a factory or poured at the site and then hoisted onto the structure.

Concrete Roof: For this survey, a poured concrete roof, often intended to bear the load of a parking garage that occupies the roof area of a building.

Conservation Feature: A feature in the building designed to reduce the usage of energy. (See Building Shell Conservation Feature, HVAC Conservation Feature, and Lighting Conservation Feature.) 
Consumption: The amount of energy used by, or delivered to, a building during a given period of time. For this report, all consumption statistics, unless otherwise noted, are site energy consumption, which excludes electrical system and district heat energy losses. In contrast, primary energy consumption takes into account the fuels that are required to produce and distribute electricity and district heat. Statistics for this report are presented on an annual basis for the 365-day period of calendar year 1989. Data on energy consumption were not collected by end uses separately. For example, although it might be known that electricity was used in some buildings for heating, the consumption of electricity reported for those buildings would typically include other uses of electricity as well (such as lighting and water heating). (See Btu, Energy Supplier, and Expenditures.)

\section{Conversion Factors: See Btu Conversion Factors and Metric Conversion Factors.}

Cooking: In this report, the use of energy for commercial or institutional food preparation. The 1989 CBECS asked specifically about "commercial or institutional cooking," which was intended to include any kitchen facility that was not part of a residence. (See Energy End Use.)

Cooling: Conditioning of room air for human comfort by a refrigeration unit (such as an air-conditioner or heat pump) or by circulating chilled water through a central cooling or district cooling system. Use of fans or blowers by themselves, without chilled air or water, is not included in this definition of cooling. (See Energy End Use, Central Cooling, Heat Pump, and HVAC.)

Cooling Degree-Days (CDD): A measure of how hot a location was over a period of time, relative to a base temperature. In this report, the base temperature is 65 degrees Fahrenheit (approximately 18 degrees Celsius), and the period of time is 1 year. The cooling degree-days for a single day is the difference between that day's average temperature and the base temperature if the daily average is greater than the base and zero if the daily average temperature is less than or equal to the base temperature. The cooling degree-days for a longer period of time is the sum of the daily cooling degree-days for the days in that period. One cooling degree-day Fahrenheit equals fiveninths of a degree-day Celsius. (See Heating Degree-Days (HDD) and Climate Zone.)

Cubic Foot: As a natural gas measure, the volume of gas contained in a cube with an edge that is 1 foot long at standard temperature and pressure (60 degrees Fahrenheit and 14.73 pounds standard per square inch.) The thermal content varies by the composition of the gas. (See Natural Gas and Btu.)

Decorative or Construction Glass: An exterior building wall material of glass decorative coverings such as glass blocks or spandrels, that are not window or vision (see-through) glass. Structural glass or glass curtain walls used on the outside of buildings are also included in this category. For this report, decorative or construction glass was included in the "Other" exterior wall material category. (See Window or Vision Glass.)

Degree-Days 1989: The total annual heating and cooling degree-days (base 65 degrees Fahrenheit) during calendar year 1989. For this report, each building was assigned to a National Oceanic and Atmospheric Administration (NOAA) Division, and the division's daily temperature averages were used to compute degree-days for 1989 . The daily temperature data were obtained from NOAA. (See Heating Degree-Days (HDD), Cooling Degree-Days (CDD), Degree-Days 45-Year Average, and NOAA Division.)

Degree-Days 45-Year Average: The average of the 45 total annual heating and cooling degree-days (base, 65 Degrees Fahrenheit) in each NOAA Division, for the years 1931 through 1975 . Computed form the Division's daily temperature averages for each year in question. Used to assign individual buildings to climate zones. (See Heating Degree-Days (HDD), Cooling Degree-Days (CDD), Degree-Days 1989, NOAA Division, and Climate Zone.)

Demand-Side Management (DSM) Programs: These are organized utility-sponsored activities that are intended to affect the amount and timing of customer electricity use.

District Chilled Water: Chilled water from an outside source used as an energy source for cooling in a building. The water is chilled in a central plant and piped into the building. Chilled water may be purchased from a utility or provided by a central physical plant in a separate building that is part of the same multibuilding facility (for 
example, a hospital complex or university). (See Energy Source, Central Physical Plant, and Multibuilding Facility.)

District Heat: Steam or hot water from an outside source used as an energy source for space heating or another end use in a building. The steam or hot water is produced in a central plant and piped into the building. The district heat may be purchased from a utility or provided by a central physical plant in a separate building that is part of the same multibuilding facility (for example, a hospital complex or university). For this report, district steam and district hot water are usually reported together as district heat. (See Energy Source, Central Physical Plant, and Multibuilding Facility.)

District Hot Water: District heat in the form of hot water. (See District Heat.)

District Steam: District heat in the form of steam. (See District Heat.)

\section{DSM: See Demand-Side Management Programs.}

Duct: A passageway made of sheet metal or other suitable material to convey air from the heating, ventilating, and cooling systems to and from the point of utilization.

Electric Baseboard: An individual space heater with electric resistance coils mounted behind shallow panels along baseboards. Electric baseboards rely on passive convection to distribute heated air to the space. (See Individual Space Heater and Baseboard.)

Electricity: Electric energy, usually measured in kilowatthours. As an energy source for this report, electric energy supplied to a building by a central utility via power lines or from a central physical plant in a separate building that is part of the same multibuilding facility. Electric power generated within a building for exclusive use in that building is specifically excluded from the definition of electricity as an energy source. (See Energy Source, Central Physical Plant, and Multibuilding Facility.)

Electricity Generation: The onsite production of electricity using electricity generators on either a regular or emergency basis. (See Electricity.)

\section{EMCS: See Energy Management and Control System (EMCS)}

Energy End Use: A use for which energy is consumed in a building. End-use estimates for nine end uses are provided in this report. The 1989 CBECS also collected data on the whether any manufacturing or electricity generation took place in the building, but separate energy consumption estimates are not available for these two end uses. (See Cooking, Cooling, Space Heating, Electricity Generation, Lighting, Manufacturing, Refrigeration, Ventilation and Water Heating.)

Energy Intensity: The ratio of energy consumption to some measure of the demand for services provided by energy. In this report, energy intensity is usually given on an aggregate basis, as the ratio of the total consumption for a set of buildings to the total service demand in those buildings. This report uses both floorspace and more complicated measures, such as floorspace-hours, to measure the demand for energy services. Energy intensities are calculated to adjust the amount of energy consumed for the effects of various building characteristics, such as size of the building, number of workers, or number of operating hours, to facilitate comparisons of energy across time, fuels, and buildings. (See Consumption.)

Energy Management and Control System (EMCS): An energy conservation feature that uses mini/microcomputers, instrumentation, control equipment, and software to manage a building's use of energy for heating, ventilation, air conditioning, lighting, and/or business-related processes. These systems can also manage fire control, safety, and security. Not included as EMCS are time-clock thermostats. (See Occupant Control of Heating and Occupant Control of Cooling.) 
Energy Source: A type of energy or fuel consumed in the building. For this report, the energy sources for which consumption and expenditures statistics are presented are electricity, natural gas, fuel oil, district heat, and district chilled water. The 1989 CBECS also collected information on the use, but not on the amounts consumed of, or spent for, these energy sources: propane, wood, coal, and active solar. (See Electricity, Natural Gas, Fuel Oil, District Heat, District Chilled Water, Liquefied Petroleum Gas (LPG), Propane, Wood, Coal, and Active Solar.)

Energy Supplier: A company that provides electricity, natural gas, fuel oil, or other sources of energy to a building. In the 1989 CBECS, only suppliers of electricity, natural gas, fuel oil, and district heat or chilled water were sent the Supplier Survey. (See Energy Source.)

\section{Envelope: See Building Shell (Envelope).}

Establishment: As defined by the Standard Industrial Classification Manual developed by the Office of Management and Budget, "an economic unit, generally, at a single physical location where business is conducted or where services or industrial operations are performed." However, "establishment" is not synonymous with "building." In this survey, respondents were asked how many establishments or organizations occupy the building (i.e., hold or lease space in it on a full-time basis).

Evaporative Cooler ("Swamp" Cooler): A type of cooling equipment using the evaporation of water to cool air. This type of equipment is commonly found in warm, dry climates. In this report, evaporative coolers are included under "Other Cooling Equipment." (See Cooling.)

Expenditures: Funds spent for the energy consumed in, or delivered to, a building during a given period of time. For this report, all expenditure statistics are presented on an annual basis, for calendar year 1989. The total dollar amount includes State and local taxes, fuel adjustment charges, system charges, and demand charges. The total dollar amount excludes merchandise, repair charges, and service charges. (See Consumption, and Energy Supplier.)

Exterior or Interior Shadings or Awnings: A covering designed to reduce the flux of light into a building. Exterior shadings or awnings include any type of shading (including architectural) or awning on the outside of the building designed to limit solar penetration. Interior shadings are drapes, venetian blinds, shades or any other means of covering a window from the inside to limit the amount of solar or thermal penetration. (See Building Shell Conservation Feature.)

Fan-Coil Unit: A type of heating and cooling distribution equipment using circulating hot or chilled water with fans. Fan-coil units have thermostatically controlled built-in fans that draw air from the room and then across finned tubes containing hot water, steam, or chilled water. The hot water, steam or chilled water can be produced by equipment within the building or be piped into the building as part of a district heating or cooling system. (See Space Heating and Cooling.)

Floors: The number of levels in the tallest section of a building, including parking areas, basements, or other floors below ground level.

Floorspace: All the area enclosed by the exterior walls of a building, including indoor parking facilities, basements, hallways, lobbies, stairways, and elevator shafts. (See Energy Source-Specific Floorspace, Gross Floorspace, and Square Footage.)

Fluorescent Lamp: A lamp made of a glass tube coated on the inside with fluorescent material. The lamp produces light by passing electricity through mercury vapor, which causes the fluorescent coating to glow or fluoresce. (See Lamp.)

Fuel: See Energy Source. 
Fuel Oil: A liquid petroleum product less volatile than gasoline, used as an energy source. In this report, fuel oil includes distillate fuel oil (No. 1, No. 2, and No. 4,), residual fuel oil (No. 5 and No. 6), and kerosene. (See Energy Source.)

Furnace: Space heating equipment consisting of an enclosed chamber where fuel is burned or electrical resistance is used to heat air directly, without using steam or hot water. The warm air is for heating, which is distributed throughout the building, typically by air ducts. (See Boiler, Ducts, Space Heating, and HVAC.)

Gallon: A volumetric measure equal to 4 quarts (231 cubic inches) used to measure fuel oil. One barrel equals 42 gallons. (See Barrel.)

Government Owned: Owned by a Federal, State, or local government agency. The building may be occupied by agencies of more than one government and may also be shared with nongovernment establishments.

HDD: See Heating Degree-Days (HDD).

Heat Pump: Heating and/or cooling equipment that, during the heating season, draws heat into a building from outside and, during the cooling season, ejects heat from the building to the outside. Heat pumps are vapor-compression refrigeration systems whose indoor/outdoor coils are used reversibly as condensers or evaporators, depending on the need for heating or cooling. (See Cooling, Space Heating, Central Cooling, and HVAC.)

\section{Heating: See Space Heating.}

\section{Heating or Reheating Coils: See Reheating Coils.}

Heating Degree-Days (HDD): A measure of how cold a location was over a period of time, relative to a base temperature. In this report, the base temperature used is 65 degrees Fahrenheit (approximately 18 degrees Celsius), and the period of time is 1 year. The heating degree-days for a single day is the difference between the base temperature and the day's average temperature if the daily average is less than the base, and zero if the daily average temperature is greater than or equal to the base temperature. The heating degree-days for a longer period of time is the sum of the daily heating degree-days for days in that period. One degree-day Fahrenheit equals five-ninths of a degree-day Celsius. (See Cooling Degree-Days (CDD), Climate Zone, and NOAA Division.)

\section{HIID: See High-Intensity Discharge (HID) Lamp.}

High-Efficiency Ballast: A lighting conservation feature consisting of an energy-efficient version of a conventional electromagnetic ballast. The ballast is the transformer for fluorescent and $\mathrm{HID}$ lamps providing the necessary current, voltage, and wave-form conditions to operate the lamp. A high-efficiency ballast requires lower power input than a conventional ballast to operate $\mathrm{HID}$ and fluorescent lamps.

High-Efficiency Lighting: As used in this report, lighting provided by high-intensity discharge (HID) lamps and/or fluorescent lamps. (See High-Intensity Discharge (HID) Lamp and Fluorescent Lamp.)

High-Intensity Discharge (HID) Lamp: A lamp that produces light by passing electricity through gas, which causes the gas to glow. Examples of HID lamps are mercury vapor lamps, metal halide lamps, and high-pressure sodium lamps. (See Lamp.)

\section{Hours of Operation: See Weekly Operating Hours.}

HVAC: An abbreviation for the heating, ventilation, and air-conditioning system; the system or systems that condition air in a building.

HVAC Conservation Feature: A building feature designed to reduce the amount of energy consumed by the heating, cooling, and ventilating equipment. The 1989 Building Characteristics Survey collected data on the presence 
of two HVAC conservation features: preventive maintenance program for the heating and cooling equipment and energy management and control systems. (See Preventive Maintenance Program for the Heating and/or Cooling Equipment, Occupant Control of Heating, Occupant Control of Cooling, Reduced Use--Off Hours, and Energy Management and Control System (EMCS).)

\section{Ice-Making Machines: See Refrigeration Equipment.}

Incandescent Lamp: A lamp that produces light by electrically heating a filament so that it glows. Included in this category are the familiar household light bulbs which screw into sockets, as well as energy-efficient incandescent bulbs such as Tungsten Halogen (spotlights), Reflector or R-Lamps (accent and task lighting), Parabolic Aluminized Reflector (PAR) lamps (flood and spot lighting), and Ellipsoidal Reflector (ER) lamps (recessed lighting). (See Lamp.)

Individual Air Conditioners in Walls or Windows: Self-contained air-conditioning units installed in either walls or windows (with heat-radiating condensers exposed to the outdoor air). These units are characterized by a lack of pipes or duct work for distributing the cool air; the units condition air only in the room or areas where they are located. (See Cooling.)

Individual Space Heater: A free-standing or self-contained unit that generates and delivers heat to a local zone within the building. The heater may be permanently mounted in a wall or floor, or may be portable. Examples of individual space heaters include electric baseboards, electric radiant or quartz heaters, heating panels, gas- or kerosene-fired unit heaters, wood stoves, and infrared radiant heaters. These heaters are characterized by a lack of pipes or duct work for distributing hot water, steam, or warm air through the building. (See Electric Baseboard.)

Insulation: A building shell conservation feature consisting of material placed between the interior of a building and the outdoor environment to reduce the rate of heat loss to the environment or heat gain from the environment. Examples include glass-wool fill and foam board. (See Roof or Ceiling Insulation, Wall Insulation, and Building Shell Conservation Feature.)

\section{Intensity: See Energy Intensity.}

Kerosene: A petroleum distillate with properties similar to those of No. 1 fuel oil, used primarily in space heaters, cooking stoves, and water heaters. In this report, no distinction is made between kerosene and fuel oil. (See Fuel Oil.)

Kilowatthour (kWh): A unit of work or energy, measured as 1 kilowatt (1,000 watts) of power expended for 1 hour. (See Btu, Electricity, and Consumption.)

Lamp: A term generally used to describe a manmade source of light. The term is often used when referring to a "bulb" or "tube." The CBECS collects data only about lamps that use electricity. (See Incandescent Lamp, Fluorescent Lamp, and High-Intensity Discharge (HID) Lamp.)

Lighting: The illumination of the interior of a building by use of artificial sources of light. (See Energy End Use.)

Lighting Conservation Feature: A building feature or practice designed to reduce the amount of energy consumed by the lighting system. The 1989 CBECS collected data on one lighting conservation feature--high-efficiency ballasts. (See High-Efficiency Ballast.)

Liquefied Petroleum Gas (LPG): Gas fuel in liquid form supplied to a building as an energy source. The fuel is usually delivered by tank trucks and stored near the building in a tank or cylinder until used. LPG contains mostly propane, but can contain such gases as butane, propylene, butylene, or ethane. For this report, any LPG reported was assumed to be propane. The 1989 CBECS did not collect consumption and expenditures data for LPG. (See Energy Source, Propane, and Natural Gas.) 


\section{LPG: See Liquefied Petroleum Gas (LPG).}

Major Energy Sources: The energy sources or fuels for which consumption and expenditures data were collected on the 1989 CBECS. These fuels or energy sources are electricity, fuel oil, natural gas, district steam, district hot water, and district chilled water. District chilled water is not included in any totals for the sum of major energy sources or fuels; all other major fuels are included in these totals.

Manufacturing: As an energy end use, any of the energy-using operations required for manufacturing/industrial processes. (See Energy End Use.)

Masonry: A general term covering wall construction using masonry materials such as brick, concrete block, stone, and tile that are set in mortar; also included in this category is stucco. This category does not include concrete panels because use of concrete panels represents a different method of constructing buildings. Concrete panels are reported separately. (See Concrete Panel.)

Metal Panel: An exterior wall construction material made of aluminum or galvanized steel panels fabricated in factories and fastened to the frame of the building to form outside walls. Pre-engineered metal buildings are also included in this category.

Metal Surfacing: Light-gauge metal sheets used for roofing.

Metric Conversion Factors: In this teport, estimates are presented in customary U.S. units. Floorspace estimates may be converted to metric units by using the relationship, 1 square foot is approximately equal to .0929 square meters. Energy estimates may be converted to metric units by using the relationship, $1 \mathrm{Btu}$ is approximately equal to 1,055 joules. (See Btu.)

Metropolitan: Buildings located within Metropolitan Statistical Areas (MSA's) as defined in the 1980 Census. Except in New England, an MSA is a county or a group of contiguous counties that contains at least one city of 50,000 inhabitants or more, or "twin cities" with a combined population of at least 50,000. The contiguous counties are included in an MSA if they are essentially metropolitan in character and are socially and economically integrated with the central city. In New England, MSA's consist of towns and cities rather than counties. (See Nonmetropolitan.)

Metropolitan Status: A building classification, either metropolitan or nonmetropolitan. (See Metropolitan and Nonmetropolitan.)

\section{MSA: See Metropolitan.}

Multibuilding Facility: A group of two or more buildings on the same site owned or operated by a single organization, business, or individual. Examples include university campuses and hospital complexes. (See Building.)

Multistage Area Probability Sample: A sample design executed in stages with geographic "clusters" of sampling units selected at each stage. This procedure reduces survey expense while maintaining national coverage.

Natural Gas: Hydrocarbon gas (mostly methane) supplied as an energy source to individual buildings by pipelines from a central utility company. Natural gas does not refer to liquefied petroleum gas or to privately owned gas wells operated by a building owner. (See Energy Source, Liquefied Petroleum Gas (LPG), and Propane.)

NOAA Division: One of the 356 weather divisions designated by the National Oceanic and Atmospheric Administration (NOAA), encompassing the United States and the District of Columbia. These divisions usually follow county borders to encompass counties with similar weather conditions. However, the NOAA Division does not follow county borders when weather conditions vary considerably within a county, as is likely to be the case when a county borders the ocean or contains high mountains. (See Climate Zone, Cooling Degree-Days (CDD), and Heating Degree-Days (HDD).) 
Nonmetropolitan: Buildings not located within Metropolitan Statistical Areas as defined in the 1980 Census. (See Metropolitan.)

Number of Workers in the Building: The number of people working in a building during the main shift on a typical workday during the year. Included in this definition are self-employed workers and volunteers. Excluded from this definition are customers, patients, and students, unless they are working for establishments in the building. Also excluded are employees who work out of the office, such as salespeople who report in, delivery people with routes, and messengers.

Occupant Control of Cooling: Control by individuals, other than maintenance personnel, of the cooling equipment in a building.

Occupant Control of Heating: Control by individuals, other than maintenance personnel, of the heating equipment in a building.

Office Equipment: A class of energy-using equipment including typewriters, copiers, cash registers, computer terminals, personal computers, printers, mainframe computer systems, and other miscellaneous office equipment. (See Energy End Use.)

Other End Uses: All energy end uses other than those specifically mentioned. (See Energy End Use.)

Owner Occupied: Having the owner or the owner's business represented at the site. A building is considered owner occupied if an employee or representative of the owner (such as a building engineer or building manager) maintains office space in the building. Similarly, a chain store is considered owner occupied even though the actual owner may not be in the building but headquartered elsewhere. Other examples of the owner's business occupying a building include State-owned university buildings, elementary and secondary schools owned by a public school district, and a post office where the building is owned by the U.S. Postal Service.

Packaged Cooling Units: See Packaged Units.

Packaged Heating Units: See Packaged Units.

Packaged Units: Units built and assembled at a factory and installed as a self-contained unit to heat or cool all or portions of a building. Packaged units are in contrast to engineer-specified units built up from individual components for use in a given building. "Packaged Units" is a term that can apply to heating equipment, cooling equipment, or combined heating and cooling equipment. Some types of electric packaged units are also called "Direct Expansion" or DX units. (See Cooling, HVAC, and Space Heating.)

Percent Cooled: The percentage of the building's square footage that is cooled to meet the comfort requirements of the occupants. For the 1989 CBECS, the point of reference for the percent cooled was the cooling season during the 12 months prior to the interview. (See Square Footage and Cooling.)

Percent Heated: The percentage of the building's square footage designed to be heated to at least 50 degrees Fahrenheit. For the 1989 CBECS, the percent heated was for the heating season during the 12 months prior to the interview. (See Total Square Footage and Space Heating.)

Percent Lit When Closed: The percentage of the building's square footage that was lit electrically during all hours other than the usual operating hours during the 12 months prior to the interview. (See Percent Lit When Open, Square Footage, and Weekly Operating Hours.)

Percent Lit When Open: The percentage of the building's square footage that was lit electrically during usual operating hours during the 12 months prior to the interview. (See Percent Lit When Closed, Square Footage, and Weekly Operating Hours.) 
Pounds (District Heat): A weight quantity of steam, also used in this report to denote a quantity of energy in the form of steam. The amount of usable energy obtained from a pound of steam depends on its temperature and pressure at the point of consumption and on the drop in pressure after consumption. (See Btu, District Steam, and District Heat.)

Preventive Maintenance Program for Heating and/or Cooling Equipment: As used in this report, a HVAC conservation feature consisting of a program of routine inspection and service for the heating and/or cooling equipment. The inspection is performed on a regular basis, even if there are no apparent problems. (See HVAC Conservation Feature.)

Primary Electricity: The energy embodied in site electricity. (See Consumption.)

\section{Primary Energy: See Consumption.}

Primary Sampling Unit (PSU): The sampling units selected at the first stage in a multistage area probability sample. A PSU typically consists of one to several contiguous counties--for example, a metropolitan area with surrounding suburban counties. (See Multistage Area Probability Sample, and Metropolitan.)

Principal Building Activity: The activity or function occupying the most floorspace in the building. The categories were designed to group buildings that have similar patterns of energy consumption. (See Building and Floorspace.)

The principal building activity categories used in this report are described below.

Assembly: signifies buildings used for the gathering of people for social, recreational, or religious activities whether in private or nonprivate meeting halls.

Education: refers to buildings that house academic or technical classroom instruction.

Food Sales: involves the retail or wholesale sale of food, such as grocery stores.

Food Service: activities that involve preparation and sale of food and beverages for consumption, such as restaurants.

Health Care: covers diagnostic and treatment facilities for both inpatient and outpatient care. Excluded from this group are skilled nursing or other residential care facilities (nursing homes). These buildings are classified as "Lodging" buildings.

Lodging: refers to buildings that offer multiple accommodations for short-term or long-term residents (including nursing homes).

Mercantile and Service: refers to buildings containing sales and displays of goods or services (excluding food).

Office: refers to buildings used for general office space, professional offices, and administrative offices.

Other: covers buildings that do not fit into any of the other named categories.

Parking Garage: refers to buildings used to park cars or other vehicles. Buildings in this category need not be totally enclosed by walls.

Public Order and Safety: describes buildings used in the preservation of law and order or safety.

Warehouse: describes buildings used to store goods, manufactured products, merchandise, or raw materials. This category includes both refrigerated and nonrefrigerated warehouses. 
Vacant: designates buildings in which more floorspace was vacant than was used for any single activity (as defined above) at the time of interview. A vacant building may have some occupied floorspace.

In graphs presentations throughout this report, the category "other buildings" includes parking garages, public order and safety buildings, and vacant buildings.

Propane: A gaseous petroleum product that liquefies under pressure; propane is a major component in liquefied petroleum gas, or LPG. Any LPG usage reported in the CBECS was assumed to be propane. (See Liquefied Petroleum Gas (LPG).)

PSU: See Primary Sampling Unit (PSU).

Quadrillion Btu: Equivalent to $1,000,000,000,000,000\left(10^{15}\right)$ Btu. (See Btu.)

Radiator: Space-heating equipment that transfers heat from steam or hot water to air by a combination of direct radiation, conduction, and convection. Typically, a radiator is a freestanding, cast-iron fixture exposed in the space it heats. (See Space Heating.)

Reduced Use--Off Hours: A conservation feature consisting of manually or automatically reducing the amount of heating or cooling produced during the hours a building is not in full use. (See Space Heating, Cooling, and Conservation Feature.)

Reflective or Shading Glass or Film: A building shell energy conservation feature consisting of tinted or reflective glass or shading films installed on the exterior glazing of a building to reduce the rate of solar penetration into the building. (See Building Shell Conservation Feature.)

Refrigerated Vending Machines: See Refrigeration Equipment.

Refrigeration: The use of energy to maintain perishable goods at a cool temperature for sale or storage. (See Energy End Use and Refrigeration Equipment.)

Refrigeration Equipment: A type of equipment such as commercial refrigeration/freezer units for the sale or storage of perishable materials; residential-type refrigerators/freezers; ice-making machines; soda or any other refrigerated vending machines; water coolers; or any other refrigeration equipment, excluding air conditioning. Freezers are designed to keep their contents below the freezing point (32 degrees Fahrenheit), and refrigeration equipment is designed to maintain the stored items below room temperature, but above the freezing point. In the 1989 CBECS, data were collected on refrigeration/freezer equipment inside and/or adjacent to the building.

Regular HVAC Maintenance: See Preventive Maintenance Program for Heating and/or Cooling Equipment.

Reheating Coils: A part of some air-conditioning systems. Electric coils in air ducts used primarily to raise the temperature of circulated air after it was over cooled to remove moisture. Some buildings report reheating coils as their sole heating source. (See Space Heating, Cooling, and Air Duct or Air-Handling Units.)

Relative Standard Error: A measure of the reliability or precision of a survey statistic. The Relative Standard Error, or RSE, is defined as the standard error of a survey estimate, expressed as a percent of the estimate. For example, an RSE of 10 percent means that the standard error is one-tenth as large as the survey estimate. (See Standard Error.)

Residential Freezers: See Refrigeration Equipment.

Residential Refrigerators: See Refrigeration Equipment. 
Roof or Ceiling Insulation: A building shell conservation feature consisting of insulation placed in the roof (below the waterproofing layer) or in the ceiling of the top floor in the building. (See Insulation and Building Shell Conservation Feature.)

\section{RSE: See Relative Standard Error.}

RSE Column Factor: An adjustment factor used to compute RSE's. For a survey estimate in a particular row and a column of a table (that is, a particular "cell"), the approximate RSE is obtained by multiplying the RSE row factor by the RSE column factor for that cell. (See Relative Standard Error and RSE Row Factor.)

RSE Row Factor: A factor used to compute RSE's. The row factor is equal to the geometric mean of the RSE's in a particular row of the main tables. For a survey estimate in a particular row and column of a table (that is, a particular "cell"), the approximate RSE is obtained by multiplying the RSE row factor by the RSE column factor for that cell. (See Relative Standard Error and RSE Column Factor.)

Sampling: The procedure used to select cases (in this survey, buildings) for interview from the population (commercial buildings in the United States). (See Multistage Area Probability Sampling.)

\section{Shadings or Awnings: See Exterior or Interior Shadings or Awning.}

Shakes: Flat pieces of weatherproof material laid with others in a series of overlapping rows as covering for roofs and sometimes the sides of buildings. Shakes are similar to wood shingles, but instead of having a cut and smoothly planed surface, shakes have textured grooves and a rough or "split" appearance to give a rustic feeling. (See Shingles, Siding, and Wooden Materials.)

Shingles: Flat pieces of weatherproof material laid with others in a series of overlapping rows as covering for roofs and sometimes the sides of buildings. Shingles are manufactured in a variety of materials including fiberglass, wood, plastic, baked clay, tile, asbestos, asphalt, and aluminum. (See Siding, Shakes, and Wooden Materials.)

Siding: An exterior wall covering material made of wood, plastic (including vinyl), or metal. Siding is generally produced in the shape of boards and is applied to the outside of a building in overlapping rows.

Site Electricity: The amount of electricity delivered to the site (building), without adjustment for the fuels consumed in generation. (See Consumption.)

Site Energy: The amount of energy delivered to the site (building); no adjustment was made for the fuels consumed to produce electricity or district sources. (See Consumption.)

Slate or Tile: A type of roofing material. Tile refers to any thin, square, or rectangular piece of baked clay, stone, or concrete used as a roofing material. Slate refers to a particular stone used for roofing.

Solely or in Combination: In the CBECS tables, a row stub accompanied by this phrase indicates overlapping categories, so that a particular building may be included in more than one line under this stub. In general, row stubs without this designation are exclusive; that is, they divide the population of buildings into distinct groups so that a particular building is represented in no more than one line under this stub.

\section{Space Conditioning: See Space Heating, Cooling, and Ventilation.}

Space Heating: The use of mechanical equipment (including wood stoves and active solar heating devices) to heat all, or part, of a building to at least 50 degrees Fahrenheit. (See Energy End Use.)

Square Footage: Floorspace, in units of square feet. One square foot is approximately equal to 0.0929 square meters. (See Floorspace and Metric Conversion.) 
Standard Error: A measure of the precision of an estimate, equal to the square root of the variance. (See Variance, Relative Standard Error (RSE), and Appendix B, "Detailed Tables.")

Steam: See District Steam.

Steam or Hot Water Radiators: See Baseboard and Radiator.

Storm or Multiple Glazing: A building shell conservation feature consisting of storm windows, storm doors, or double- or triple-paned glass that are placed on the exterior of the building to reduce the rate of heat loss. (See Building Shell Conservation Feature.)

Synthetic or Rubber Roofing: A layer (either single- or multi-ply) of heavy gauge plastic or rubber used for roofing.

Thermostat: A device that adjusts the amount of heating and cooling produced and/or distributed by automatically responding to the temperature in the environment.

Tinted Glass: See Reflective or Shading Glass or Film.

Total Square Footage: Square footage of floorspace summed or aggregated over all buildings in a category (such as all office buildings in the United States). In this survey, aggregate square footage was estimated by multiplying each building's square footage by its weight, then summing over all sample buildings of interest to represent nationwide totals. (See Floorspace and Weight.)

Trillion Btu: Equivalent to 1,000,000,000,000 (102) Btu. (See Btu.)

Utility-Sponsored Conservation Program: Any program sponsored by an electric and/or natural gas utility to review operating practices, equipment and construction features in buildings and advise on ways to increase the energy efficiency of buildings. Also included are utility-sponsored demand-side management programs to encourage the use of more energy-efficient equipment or practices. Included in this survey were programs to improve the energy efficiency in the lighting system or building equipment, or the thermal efficiency of the building shell. (See Demand-Side Management (DSM) Programs.)

Vacant: As a principal building activity, the designation for a building in which most of the floorspace was not occupied by any tenant or establishment. A vacant building may contain occupants who are using up to 50 percent of the floorspace. The CBECS also measures vacancy in terms of the fraction of space vacant within an individual building and the fraction of time the building was in use. For all buildings, data were collected on the percent of floorspace vacant three or more months, and on the number of months the building was in use. (See Principal Building Activity.)

Variance: A measure of the variability of a set of observations that are subject to some chance variation, equal to the expected squared difference between a single observation and the average of all possible observations obtained in the same manner. The variance is the square of the standard error of estimates. For statistics presented in this report, the variance indicates the likely difference between the value computed from the CBECS sample and the average of the values that could have been computed from all possible samples that might have been obtained by the same sample selection process. (See Standard Error and Appendix B, "Detailed Tables.")

Ventilation: The circulation of air through a building to provide fresh air to the occupants, and to deliver heating and cooling to the occupied spaces.

Vintage: The year of origin or age. As used in the CBECS report, the year of construction for the building, as in "building vintage," or the age of the central chillers or packaged refrigeration units, as in "vintage of refrigeration equipment." (See Year Constructed, Central Chillers, and Packaged Units.) 
Wall Insulation: A building shell conservation feature consisting of insulation placed between the exterior and interior walls of a building. (See Insulation and Building Shell Conservation Feature.)

\section{Warm-Air Furnace: See Furnace.}

Water Coolers: See Refrigeration Equipment.

Water Heating: The use of energy to heat water for purposes other than space heating. (See Energy End Use.)

Weather Stripping or Caulking: A building shell conservation feature that includes any material placed between the door or window and the door frame or window frame to reduce the rate of loss of heat or cold caused by air infiltration. (See Building Shell Conservation Feature.)

Weekly Operating Hours: The number of hours per week that a building is used, excluding hours when the building is occupied only by maintenance, security, or other support personnel. For buildings with a schedule that varied during the year, "weekly operating hours" refers to the total weekly hours for the schedule most often followed. If operating hours varied throughout a building, the usual operating hours of the largest business in the building (based on square footage) determined the operating hours for the building.

Weight: The number of buildings in the United States that a particular sample building represents. To estimate the total value of an attribute (such as square footage) in the U.S. commercial building population as a whole, each sample building's value is multiplied by the building's weight. Summing the weighted sample values provides an estimate of the nationwide total. (See Multistage Area Probability Sample.)

Window or Vision Glass: An exterior wall construction material made of glass that can be seen through from the inside of the building--the glass especially found in windows. Walls that are glass covered or constructed of glass material, but cannot be seen through, are excluded from this category. (See Decorative or Construction Glass.)

Wood: As an energy source, wood logs, chips, or wood products that are used as fuel. In the 1989 CBECS, information about the use of wood as fuel in commercial buildings was obtained, but consumption and expenditures data for wood were not collected. (See Energy Source.)

Wooden Materials: Wood shingles, wood shakes, or other wooden materials used as roofing materials. (See Shingles and Shakes.)

Workers: See Number of Workers in the Building.

Year Constructed: The year in which the major part or the largest portion of a building was constructed. 